\title{
An X-ray survey of low-mass stars in Trumpler 16 with Chandra ${ }^{\star}$
}

\author{
J. F. Albacete-Colombo ${ }^{1,2}$, F. Damiani ${ }^{2}$, G. Micela ${ }^{2}$, S. Sciortino ${ }^{2}$, and F. R. Harnden, Jr. ${ }^{3}$ \\ ${ }^{1}$ Centro Universitario Regional Zona Atlántica - Univ. COMAHUE, Monseñor Esandi y Ayacucho (CP 8500), Viedma, Argentina \\ e-mail: donfaca@gmail.com \\ 2 INAF - Osservatorio Astronomico di Palermo, Piazza del Parlamento 1, 90134 Palermo, Italy \\ 3 AF Smithsonian Astrophysical Observatory, 60 Garden St., Cambridge, MA 02138, USA
}

Received 17 April 2008 / Accepted 23 July 2008

\section{ABSTRACT}

\begin{abstract}
Aims. We identify and characterize low-mass stars in the $\sim 3$ Myr old Trumpler 16 region by means of a deep Chandra X-ray observation, and study their optical and near-IR properties. We compare the X-ray activity of Trumpler 16 stars with the known characteristics of Orion and Cygnus OB2 stars.

Methods. We analyzed a 88.4 ks Chandra ACIS-I observation pointed at the center of Trumpler 16. Because of diffuse X-ray emission, source detection was performed using the PWDetect code for two different energy ranges: $0.5-8.0 \mathrm{keV}$ and $0.9-8.0 \mathrm{keV}$. Results were merged into a single final list. We positionally correlated X-ray sources with optical and 2MASS catalogs. Source events were extracted with the IDL-based routine ACIS-Extract. X-ray variability was characterized using the Kolmogorov-Smirnov test and spectra were fitted by using XSPEC. The X-ray spectra of early-type, massive stars were analyzed individually.

Results. Our list of X-ray sources consists of 1035 entries, 660 of which have near-IR counterparts and are probably associated with Trumpler 16 members. From near-IR, color-color, and color-magnitude diagrams we compute individual masses of stars and their $A_{\mathrm{v}}$ values. The cluster median extinction is $A_{\mathrm{v}}=3.6 \mathrm{mag}$, while OB-type stars appear less absorbed, having $A_{\mathrm{v}}=2.0 \mathrm{mag}$. About $15 \%$ of the near-IR counterparts show disk-induced excesses. X-ray variability is found in 77 sources, and typical X-ray spectral parameters are $N_{\mathrm{H}} \sim 5.37 \times 10^{21} \mathrm{~cm}^{-2}$ and $k T \sim 1.95 \mathrm{keV}$. The OB stars appear, softer with a median $k T \sim 0.65 \mathrm{keV}$. The median $\mathrm{X}$-ray luminosity is $6.3 \times 10^{30} \mathrm{erg} \mathrm{s}^{-1}$, while variable sources show a larger median $L_{\mathrm{x}}$ value of $13 \times 10^{30} \mathrm{erg} \mathrm{s}^{-1}$. OB-stars have an even higher median $L_{\mathrm{x}}$ of $80 \times 10^{30} \mathrm{erg} \mathrm{s}^{-1}$, about 10 times that of the low-mass stars.

Conclusions. The Trumpler 16 region has a very rich population of low-mass X-ray emitting stars. A large fraction of its circumstellar disks have survived the intense radiation field of its massive stars. Stars with masses 1.5-2.5 $M_{\odot}$ display X-ray activity similar to the Cyg OB2 stars, but much less intense than observed for Orion nebula cluster members.
\end{abstract}

Key words. stars: formation - stars: early-type - stars: pre-main sequence - X-rays: stars

\section{Introduction}

The Carina nebula region (NGC 3372) is one of the most massive star formation regions of the Galaxy. It is associated with a giant HII region spanning about $4 \operatorname{deg}^{2}$ of the sky and is bisected by a prominent $\mathrm{V}$-shaped dark gas and dusty lane. This prominent young structure is not as compact as some of the other young galactic clusters, but seemingly to be related to a spiral feature. In this direction, we are looking almost tangentially toward the now recognized Carina-Sagittarius spiral arm, at the edge of a giant molecular cloud extending over about $130 \mathrm{pc}$, which has a content in excess of $5 \times 10^{5}$ solar masses (Grabelsky et al. 1988). The concentration of massive stars (i.e. $M \geq 20 M_{\odot}$ ) interacts with the parent giant molecular cloud of the region, leading to triggered star formation events on intermediate to lower masses (e.g. Smith et al. 2004).

This region harbors several open clusters and/or star concentrations (Trumpler 14-16; Collinder 228 and 232; Bochum 10 and 11) containing more than 60 known O-type stars (Feinstein 1995). Large cavities within the giant molecular cloud are supposed to be carved out by the $\operatorname{Tr} 14$ and 16 open clusters, which contain most of massive stars of the region. In particular, Tr 16

\footnotetext{
* Full Tables $1-3$ are only available in electronic form at http://www . aanda. org
}

includes three rare main-sequence $\mathrm{O} 3$ stars, the Wolf-Rayet (WR) star HD 93162 and the famous luminous blue variable (LBV) $\eta$ Carinae. There is a historical controversy about the distance and age of $\operatorname{Tr} 14$ and $\operatorname{Tr} 16$ (Walborn 1995). For instance, from extensive spectroscopy and photometry Massey \& Johnson (1993) find $3.2 \mathrm{kpc}$ for both clusters. However, photometric studies are strongly affected by differential extinction in the region and peculiar reddening, so the derived distance are different. An example is the Carraro et al. (2004) work, who compute distances of $4.0 \mathrm{kpc}$ and $2.5 \mathrm{kpc}$, for different $R=A_{\mathrm{v}} / E(B-V)$ values (3.48 and 4.16 for $\operatorname{Tr} 16$ and $\operatorname{Tr} 14$ regions). A more reliable distance $(2250 \pm 180 \mathrm{pc}$ ), was derived from proper motion and Doppler velocities of the expanding $\eta$ Carinae Homunculus using HST-STIS ${ }^{1}$ observations (Davidson \& Humphreys 1997). Recent work (Tapia et al. 2003) derives a common distance $\mathrm{DM}=12.14(2.7 \mathrm{kpc})$ and an age between $\sim 1 \mathrm{Myr}$ and $3 \mathrm{Myr}$, for $\operatorname{Tr} 14$ and $\operatorname{Tr} 16$, respectively. For this study, we adopt a distance for Trumpler 16 of $2250 \mathrm{pc}$ and an age of $3 \mathrm{Myr}$. This young age agrees with the Smith et al. (2000) results, which report the existence of several embedded IR sources where star formation might be active. Also, DeGioia-Eastwood et al. (2001) confirm clear evidence of pre-main sequence (PMS) stars in the region,

\footnotetext{
${ }^{1}$ Data from Hubble Space Telescope (HST) with the Space Telescope Imaging Spectrograph (STIS).
} 
while Brooks et al. (2001) have identified two compact HII regions possibly linked to very young O-type stars. Finally, Hägele et al. (2004) report a compact cluster of infrared PMS-stars in $\operatorname{Tr} 16$.

Of the existing methods of identifying young stellar populations, the use of X-ray emission is perhaps the least biased (Feigelson et al. 2002). While in main-sequence (MS) stars, from late $\mathrm{A}$ to $\mathrm{M}$ dwarfs, $\mathrm{X}$-rays are believed to originate from the hot coronal gas that is heated by stellar dynamo magnetic fields (Maggio et al. 1987), for late type Pre-MS stars (T Tauri stars (TTSs)) X-ray emission is attributed to solar-like coronal activity but elevated by a factor of $10^{3}-10^{4}$ (Feigelson \& Montmerle 1999). Several authors suggested the possibility of detecting early PMS objects through their hard X-ray emission escaping the highly obscured regions (see Walter 1992; Kamata et al. 1997; Hofner \& Churchwell 1997; Hofner et al. 2002). Recently, X-ray surveys have been successful in identifying the young and pre-MS population in star-forming regions, including: $i-$ deeply embedded Class I young stellar objects (YSOs); $i$ - low-mass T Tauri PMS stars; iii- intermediate-mass Herbig Ae/Be PMS stars; and $i v-$ zero-age MS stars. Moreover, X-ray emission from low-mass PMS stars usually exhibits a strong variability that helps to confirm membership.

In the last decade, X-ray observations of young stars on star-forming regions were intensified thanks to the high spatial resolution and the improved broad-band ([0.2-12.0] and $[0.5-10.0] \mathrm{keV}$ ) effective area of the XMM-Newton and Chandra satellites. A first X-ray survey in the Carina region by Albacete Colombo et al. (2003) was performed on the basis of two early XMM-Newton observations (rev \#115 and \#116) centered on $\eta$ Carinae. Because of the spatial resolution of the EPIC ${ }^{2}$ camera and relatively short exposure time of the observations $(\sim 35 \mathrm{ks})$, they detected only $80 \mathrm{X}$-ray sources, most of them related to the massive OB-type stars with $L_{\mathrm{x}} \sim 10^{32}-10^{34} \mathrm{erg} \mathrm{s}^{-1}$. Before the observation used here, three Chandra observations were obtained in this region, two (obsId 50 and 1249) in the timed exposure mode, and the third (obsId 51) in the continuous clocking mode, which produces no image. Using only observation obsId 1249, Evans et al. (2003) presented luminosities and hardness ratios of the hot stars in Tr 16, and part of Tr 14. Lowresolution X-ray spectra of luminous sources were discussed by Evans et al. (2004); however, the short exposure time of such an observation $(\sim 9.5 \mathrm{ks})$ was a serious limitation for the study of intermediate- and low mass stellar population in the region. This limitation exists even if obsId. 50 and obsId. 1249 are combined (Sanchawala et al. 2007), reaching completeness just at X-ray luminosity $\left(L_{\mathrm{X}}\right)$ of $\sim 7 \times 10^{31} \mathrm{erg} \mathrm{s}^{-1}$, i.e. the X-ray emission level typical of single $\mathrm{O}$ - and early B-type stars.

In this paper we present results of the analysis of the deepest $\mathrm{X}$-ray observation ever done in this region ( $\sim 90 \mathrm{ks})$. Section 2 gives details on the observation and data-reduction procedures. Section 3 explains the method used to detect the sources, photon extraction and the construction of the catalog. In Sect. 4 we present results of the cross-correlation with existing near-IR and optical catalogs of objects and their characterization based on their color-color (CC) and color-magnitude (CM) diagrams. Section 5 presents a statistical study of variability in the X-ray domain. Section 6 deals with results of the analysis of extracted X-ray spectra. In Sect. 7 we discuss X-ray luminosities of stars and compare them statistically with the X-ray source population of ONC and Cygnus OB2 star-forming regions. In Sect. 8 we

\footnotetext{
2 European Photon Image Camera has about six times less spatial resolution than Chandra ACIS-I camera.
}

discuss X-ray and stellar parameter of O- and early B-type stars. Finally, in Sect. 9 we give a summary and draw conclusions of the paper.

\section{The X-ray observations}

Trumpler 16 was observed with the ACIS detector on board the Chandra X-ray Observatory (CXO) (Weisskopf et al. 2002) on 2006 August $31^{3}$ (obsId 6402), as part of the Guaranteed Time Observation (GTO) Chandra program. The total effective exposure time was $88.4 \mathrm{ks}$. The data were acquired in VERY FAINT mode, to ease filtering of non-X-ray events, with six CCD turned on, the four comprising the ACIS-I array [0-3], plus CCDs 6 and 7, part of ACIS-S. However, data from the last two CCDs not are used in the following because of the degraded point spread function (PSF) and reduced effective area resulting from their large distance from the optical axis. The ACIS-I $17^{\prime} \times$ $17^{\prime}$ field of view (FOV) is covered by 4 chips, each with $1024 \times$ 1024 pixels (scale $0.49^{\prime \prime} \mathrm{px}^{-1}$ ). The observation was pointed toward RA $=10^{\mathrm{h}} 44^{\mathrm{m}} 47.93^{\mathrm{s}}$ and $\operatorname{Dec}=-59^{\circ} 43^{\prime} 54.21^{\prime \prime}$, chosen to maximize the number of stars in the FOV and close to the optical axis, but also including most of the OB stars of the cluster. Figure 1-left shows Trumpler 16 as seen in X-rays by our ACIS-I observation.

Most of the observed X-ray sources in Fig. 1-left are very likely located toward the central part of Trumpler 16, spatially constrained by the dark V-shaped dust lane of the Carina region (Brooks et al. 1998). However, note the small X-ray source concentration inside this dark structure, located towards the south east part of Fig. 1. Deep near-IR observation are needed to reveal counterparts of such population. We defer to a forthcoming paper the use of some public HST - WFPCII $^{4}$ observations to find signatures of star-environment interactions for some of the $\mathrm{X}$-ray sources.

\subsection{Data reduction}

Data reduction, starting with the Level 1 event list provided by the pipeline processing at the $\mathrm{CXO}$, was performed using CIAO 3.3.0.1 $1^{5}$ and the CALDB 3.1.0 set of calibration files. We produced a level-2 event file using the ACIS_PROCESS_EVENT CIAO task, taking advantage of the VF-mode enhanced background filtering and only retaining events with grades $=0,2-4,6$ and status $=0$. Photon energies were corrected for the time dependence of the energy gain using the CORR_TGAIN CIAO task. Intervals of background flaring were searched for, but none found. We hereafter assume a non-variable background. To improve the sensitivity to faint sources, given the spectrum of the background and that of typical sources, we filtered out events outside the [500:8000] eV energy band.

\section{Data analysis}

We built images in the three band-passes $0.5-1.5 \mathrm{keV}$ (soft), 1.5-2.2 keV (medium), and 2.2-8.0 keV (hard). Before the color-coded image combination, we corrected single bandimages by variations in exposure, sensitivity, and vignetting, by computing and dividing with its respective exposure maps. We constructed a color-coded X-ray image of the region by composition of the three soft (red), medium (green), and hard (blue)

\footnotetext{
3 Observation start date is JD 273350372.4528.

${ }^{4}$ Hubble Space Telescope - Wide Filed Planetary Camera 2.

5 http://cxc.harvard.edu/ciao/
} 

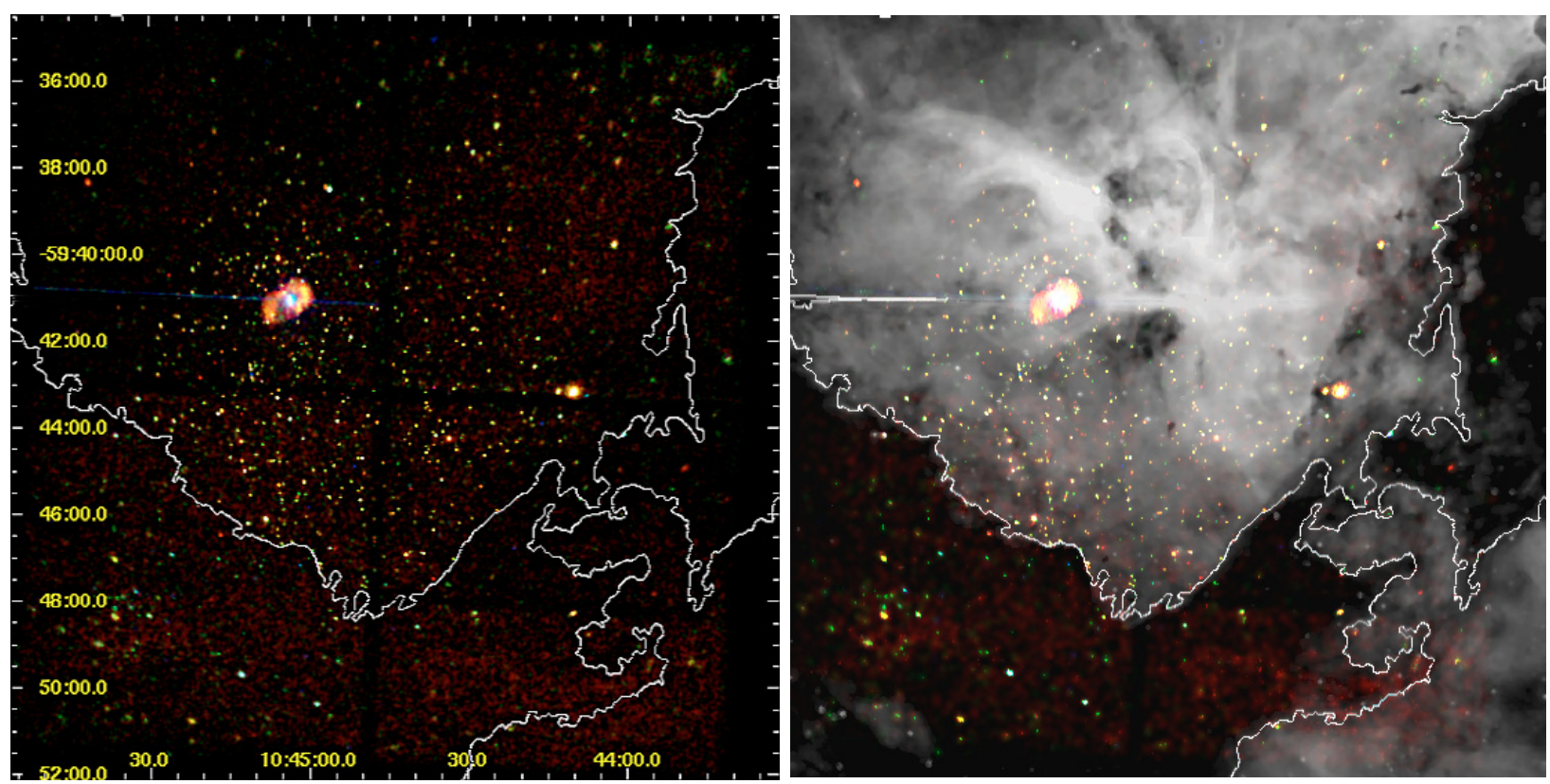

Fig. 1. Left: color-coded ACIS-I image of the $17^{\prime} \times 17^{\prime}$ field in Trumpler 16 (see color version in the electronic edition). Kernel smoothing was applied to highlight point sources. Energy bands for the RGB image are [0.5:1.5], [1.5:2.2], and [2.2:8.0] keV for the red, green, and blue colors, respectively. Contour lines show the spatial distribution of the dark V-shaped dust lane. Right: ACIS-I image composed with a $\mathrm{H}_{\alpha}$ image. This image clearly show the sharp cut, along the V-shape structure, in the spatial distribution of X-ray sources.

images (see Fig. 1). This image is a $17 \times 17$ arcmin field that comprises the center of $\operatorname{Tr} 16$ and marginally the south east part of $\operatorname{Tr} 14$. In addition to revealing a huge number of X-ray pointlike sources with different color-energies, inspection of Fig. 1 suggests the presence of soft diffuse X-ray emission in the region. In particular, Townsley (2006) use a 57 ks ACIS-I observation centered on $\operatorname{Tr} 14$ to study the diffuse X-ray emission in such a region and in the north west part of $\operatorname{Tr} 16$. They explain the diffuse X-ray emission as likely arising from the fast O-star winds that shock and thermalize the surrounding medium. Because homogeneous data analysis techniques are required to do justice to a comparison of diffuse X-ray emission in this dataset with that of $\operatorname{Tr} 14$, this study will be presented in a forthcoming paper.

\subsection{X-ray source detection}

Source detection was performed with the Palermo Wavelet Detection code, PWDetect ${ }^{6}$ (Damiani et al. 1997b). It analyzes the data at different spatial scales, allowing the detection of both point-like and moderately extended sources, and efficiently resolving close source pairs. The most important input parameter is the detection threshold (SNR), which we established from the relationship between background level of the observation and expected number of spurious detections due to Poisson noise ${ }^{7}$.

Diffuse, soft $(\sim 0.2-1.2 \mathrm{keV}) \mathrm{X}$-ray emission has already been identified in this region (Corcoran et al. 1995; Townsley 2006) and causes different (non-uniform) X-ray background levels across the FOV of our observation. The best way of reducing the diffuse emission contribution is to discard soft photons in the detection procedure. We computed background (BKG) levels in four different energy ranges: $0.5-8.0 \mathrm{keV}, 0.7-8.0 \mathrm{keV}$, 0.9-8.0 keV, and 1.1-8.0 keV. Since exposure maps are needed

\footnotetext{
6 See

http://wWw. astropa.unipa.it/progetti_ricerca/PWDetect

7 This last quantity was determined from extensive simulations of source-free fields (see Damiani et al. 1997a).
}

by the source detection algorithm, we used event files in these energy bands as input to the CIAO tool MKEXPMAP and assumed a monochromatic spectrum $(k T=2.0 \mathrm{keV})^{8}$. The background level for each energy band was computed as the average of values measured in three circular regions free of sources. If we accept 10 spurious detections in the ACIS FOV ${ }^{9}$, mean computed background levels can be translated into different SNR thresholds for each energy band, i.e.: 4.7, 4.65, 4.6, and 4.55. These input parameters for PWDetect result in different numbers of detected sources in each energy band, for instance, 1266 sources in the $0.9-8.0 \mathrm{keV}$ band, more than detected in the "canonical" $0.5-8.0 \mathrm{keV}$ band (1214 sources). This is consistent with the effect of diffuse soft X-ray emission in masking weak sources.

A careful visual inspection was performed on the 0.5-8.0 keV and $0.9-8.0 \mathrm{keV}$ source lists. We manually rejected 242 and 271 detections respectively, considered spurious either because they were produced by different instrumental artifacts (e.g. CCD gaps, detector edges, false detections along the readout trails), or since they resulted from the "fragmentation" into discrete sources of the extended emission making a toroidal ring around the LBV star $\eta$ Carinae (Fig. 1). Furthermore, examination of afterglow ${ }^{10}$ contamination led us to discard 29 and 20 false detections. The final source list was constructed by merging both catalogs: the list of 943 sources detected in the $0.5-8.0 \mathrm{keV}$ band and the 1004 sources detected in the $0.9-8.0 \mathrm{keV}$ band. Merging was performed using a criterion of maximum detection significance. The two catalogs coincide in 798 sources, consisting of 322 sources with $\mathrm{Sig}_{0.5-8.0}>\mathrm{Sig}_{0.9-8.0}$, while for the remaining 476 sources $\mathrm{Sig}_{0.9-8.0}>\mathrm{Sig}_{0.5-8.0}$ (where $\mathrm{Sig}_{x-y}$ is the detection significance

\footnotetext{
${ }^{8}$ http://asc.harvard.edu/ciao/download/doc/ expmap_intro.ps

9 See reasons of this choice in Albacete Colombo et al. (2007).

10 Afterglow is defined as the residual charge from the interaction of a cosmic ray with the CCD. If afterglow events are not removed from the data, they can result in the spurious "detection" of faint sources.
} 
in the $x-y$ energy band). Sixty-one sources were detected only in the $0.5-8.0 \mathrm{keV}$ band, while 176 are exclusive to the 0.9-8.0 keV band and would have otherwise remained hidden because of the diffuse emission. This procedure led to a total number of $1035 \mathrm{X}$-ray sources in the entire field, which we analyze here.

\subsection{Photon extraction}

Even with the high spatial resolution of the Chandra ACIS-I camera, the high source density in Trumpler 16, source photon extraction is not an easy task. Although circular regions would contain a relatively large fraction of the PSF for almost all source photons, the extended wings of the PSF mean that very large regions would be needed, incurring in the risk of contamination from nearby sources. Moreover, the resulting inclusion of a large number of background events would reduce the signalto-noise of weak sources. On the other hand, extraction from regions that are too small may reduce the photon statistics for further spectral and timing analysis. To address these issues, we decided to use ACIS EXTRACT (AE) v3.79 (Broos et al. 2002), an IDL-based package that makes use of TARA ${ }^{11}$, CIAO, and FTOOLS ${ }^{12}$ software.

This task reduces the problem of accounting for nonGaussian shapes of the local PSF by calculating the shape of the PSF model at each individual source's position. For some sources, the background level is affected by the extended PSF wings of the bright sources ( $\eta$ Carinae and WR25) in the FOV. AE computes source background locally, by defining background extraction regions as circular annuli with inner radii 1.1 times the maximum distance between the source and the $99 \%$ PSF contour, and outer radii defined so that the regions contain more than 100 "background" events. To exclude contamination of the regions by nearby sources, background events are taken from a "Swiss cheese" image that excludes events within the inner annuli radii of all the 1035 sources.

The AE source extraction was performed using a PSF model that contains a specified fraction of source events $\left(f_{\mathrm{PSF}}\right)$. Generally, we choose $f_{\mathrm{PSF}}=90 \%$, and computed the contours from the PSF for a mono-energetic source with $E=$ $1.49 \mathrm{keV}$. For $9.9 \%$ of the sources in the denser parts of the Trumpler 16 field this fraction was reduced to avoid contamination with other nearby sources, in the most extreme cases down to $f_{\mathrm{PSF}} \sim 50 \%$ (just 3 sources).

Following AE science hints, we then refined the initial source positions computed by PWDetect ${ }^{13}$ by correlating the source images with the model of local PSF computed by AE libraries. This procedure was only used for those sources lying at off-axis larger than 5 arcmin (316 sources), while for the rest of the source ( 719 sources) we simply adopted mean photon positions ${ }^{14}$. AE also estimates local background spectra, computes redistribution matrix files (RMFs) and auxiliary response files (ARFs), constructs light curves, performs Kolmogorov-Smirnov variability tests, and computes photometry in 14 different energy bands. Results of AE procedure appears in Table 1, which lists the source number in Col. (1); name according to CXC naming convention ${ }^{15}(2)$; sky position (RA and Dec J2000) $(3,4)$

\footnotetext{
${ }^{11}$ http://www . astro.psu.edu/xray/docs/TARA/

12 http://heasarc.gsfc.nasa.gov/docs/software/ftools/

13 PWDetect assumes a symmetric PSF.

14 Please follow Acis-Extract technical procedures at website

http://www . astro.psu.edu/xray/docs/TARA/ae_users_guide

15 http://cxc.harvard.edu/cdo/scipubs.html
}

with relative uncertainty (5); off-axis angle $(\theta)(6)$; significance of the detection (Sig.) from PWDetect analysis (7); the source extraction area (8); the PSF fraction within the extraction area, assuming $E=1.49 \mathrm{keV}$ (9); the background-corrected extracted source counts in the $0.5-8.0 \mathrm{keV}$ band (NetCnts) (10); the count rates $\left(\mathrm{CR}=\mathrm{NetCnts} /\right.$ Exptime $\left./ \mathrm{PSF}_{\text {frac }}\right)$ in three spectral bands: 0.5-8.0 keV, 0.5-2.0 keV and 2.0-8.0 keV, (11-13); source photon quantiles at 25,50 , and $75 \%$ percent in Cols. 14 to 16 (see Sect. 3.3); the median photon energy $\left(\overline{E_{x}}\right)$ in (17). Column 18 is the $\log \left(P_{\mathrm{ks}}\right)$ Kolmogorov-Smirnov probability of non-variability (see Sect. 5), and in (19) there are flags from PWDetect detection code.

\subsection{X-ray hardness ratios}

A commonly used tool for exploring the spectral properties of sources with low photon statistics is the hardness ratio (e.g. Schulz et al. 1989; Prestwich et al. 2003). In this conventional method, the full energy range is divided into two or three subbands and the detected source photons are counted separately in each band. Most popular definitions for a single hardness ratio (HR) exist on the basis of only two energy sub-bands: $i-\mathrm{HR}=\mathrm{H} / \mathrm{S}$ or $i i-\mathrm{HR}=(\mathrm{H}-\mathrm{S}) /(\mathrm{H}+\mathrm{S})$. By these definitions, $\mathrm{HR}$ is very sensitive to small changes (i.e. statistical fluctuations) in the number of photons falling in each band. The requirement of total counts in the full energy band is at least 40 photons (just $36 \%$ of our sources satisfy this constraint). Above this limit, HR becomes a "reliable method" of estimating the real hardness of sources (Albacete Colombo et al. 2007).

An improved method to resolve this limitation is based on the quantile analysis (Hong et al. 2004). Instead of working with predetermined energy bands, we determine the energy $E^{x}$ below which the net counts is $x \%$ of the total counts of the source. We define quantile $Q_{x}$ as $\frac{E^{x}-E_{\min }}{E_{\max }-E_{\min }}$, where in our study $E_{\min }=$ $0.5 \mathrm{keV}$ and $E_{\max }=8.0 \mathrm{keV}$. We computed the median $Q_{50}$ values and quartiles $Q_{25}$ and $Q_{75}$ and give values in Table 1. A minor inconvenience of this method is that for a given spectrum, various quantiles cannot be considered independent variables, unlike the counts in different energy bands. However, Hong et al. (2004) overcome this problem by considering the $\log \left(Q_{50} /\left(1-Q_{50}\right)\right)$ vs. $3\left(Q_{25} / Q_{75}\right)$ plane. Based on an extensive set of simulated spectra, they predict the loci of models in this plane. We used a set of absorbed thermal models with plasma temperatures of $0.2,0.5,1,2,4,10 \mathrm{keV}$, and $N_{\mathrm{H}}$ equal to $10^{20}$, $10^{21}, 0.4 \times 10^{22}, 10^{22}, 4 \times 10^{22}$, and $10^{23} \mathrm{~cm}^{-2}$. Note that the spectrum changes from soft to hard as one goes from left to right in the diagram (see Fig. 2).

In a statistical sense, a comparison between sources in the grid models suggest typical $N_{\mathrm{H}}$ and $k T$ values distributed around $0.8 \times 10^{22} \mathrm{~cm}^{-2}$ and $1.5 \mathrm{keV}$, but dispersed within grid models of $N_{\mathrm{H}}=0.4-1.0 \times 10^{22} \mathrm{~cm}^{-2}$ and $k T=1-3 \mathrm{keV}$, respectively. The absence of a systematic difference between the position of sources with and without a 2 MASS identification on the hardness-ratio plane is probably caused by a combination of two observational bias: ( $i$ ) related to the limiting magnitude of the 2MASS photometry $\left(K_{\mathrm{s}}^{\text {limit }} \sim 14.3 \mathrm{mag}\right)$; and (ii) the X-ray sensitivity of the Chandra data $\left(f_{\mathrm{x}}^{\text {limit }} \sim 10^{-14.5} \mathrm{erg} \mathrm{s}^{-1} \mathrm{~cm}^{-2}\right.$ ). However, it is not clear from the observational point of view, i.e. throughout the near-IR and X-ray flux source distributions, that a fraction of faint stellar X-rays sources would not have 2MASS counterparts. Deeper near-IR and X-ray observations are needed to unveil this issue. 


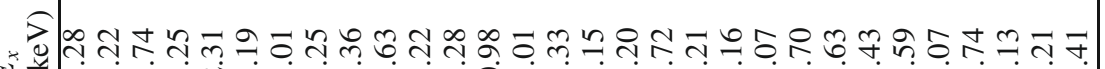

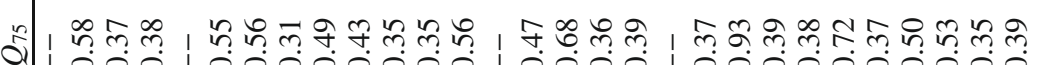
$\sqrt{3}$

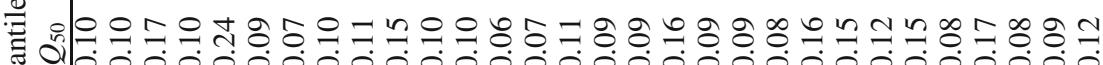

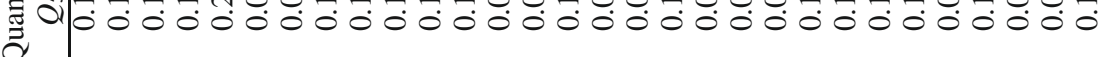

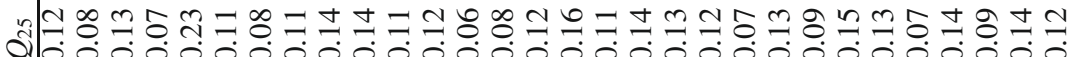

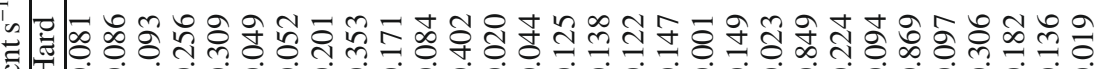
ul $00-000000000000000000000000000$ $\frac{0}{x}$

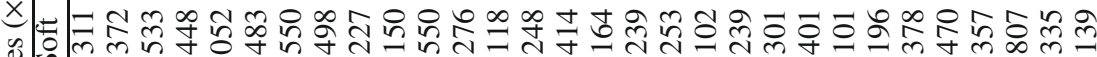

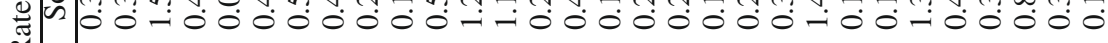
u|0.

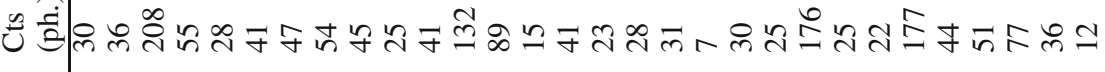

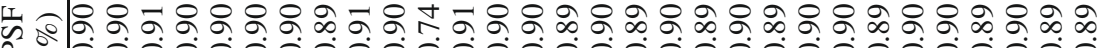
$5=0.00000000000000000000000000000$

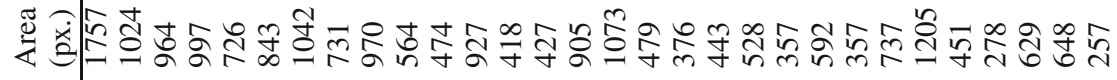

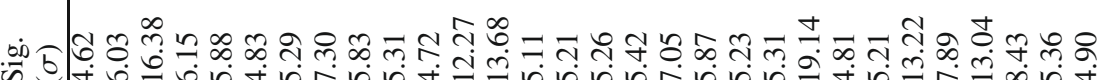

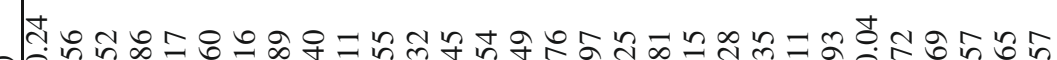

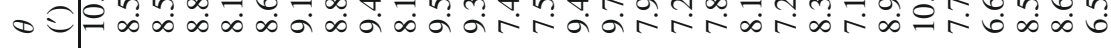

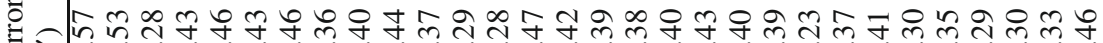

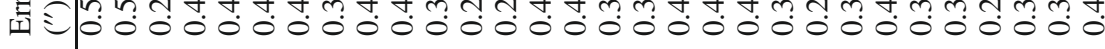

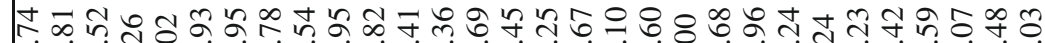

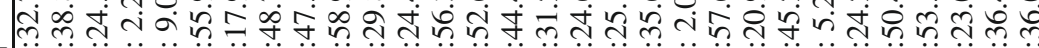

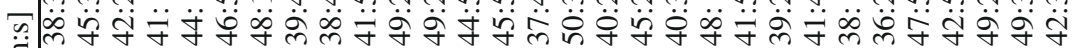

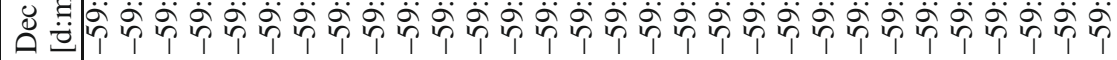

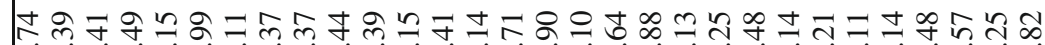

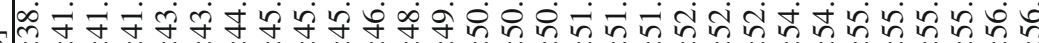

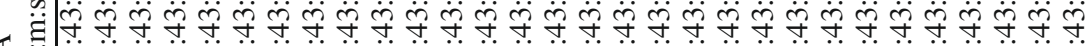

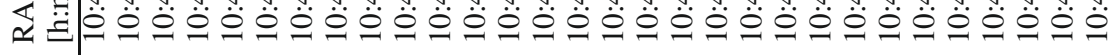

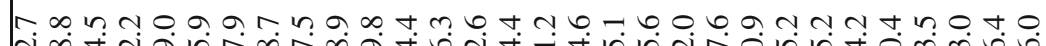

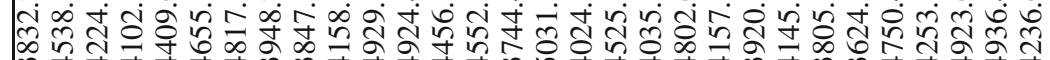

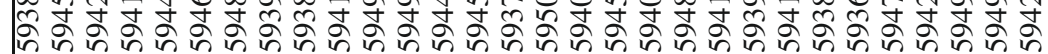

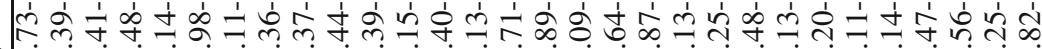

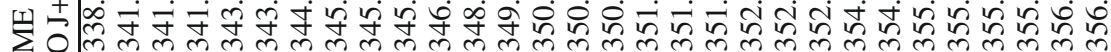

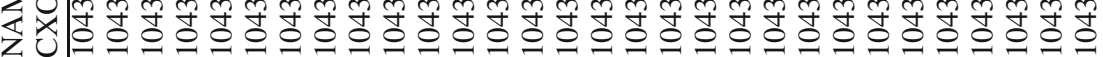




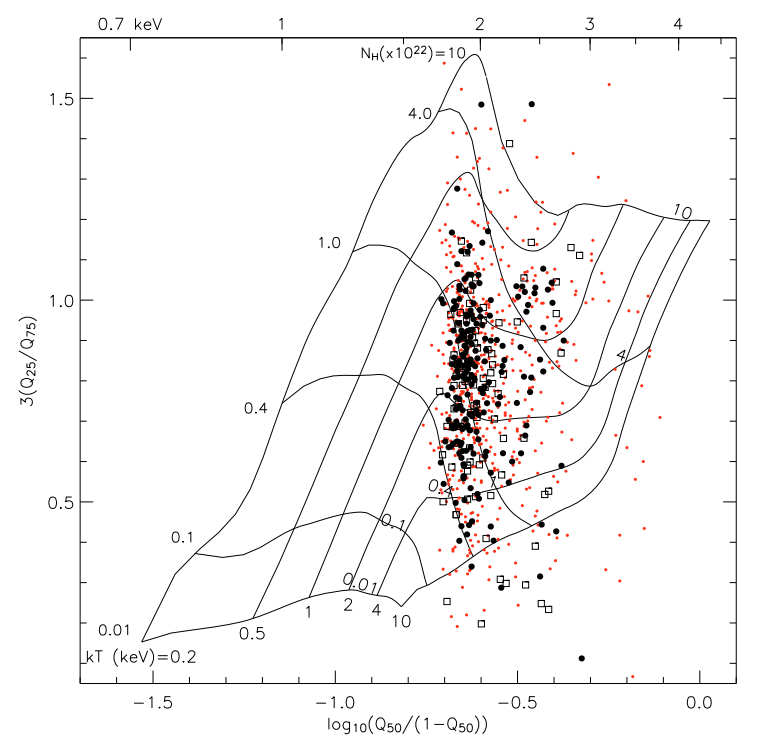

Fig. 2. Quantile color-color diagram (QCCD). The energy scale in the top $X$-axis shows the median energy values $\left(Q_{50}\right)$. The grid pattern represents the location of simulated spectra in the diagram (Hong et al. 2004). Note: filled circles and open boxes refer to X-ray sources with and without 2MASS counterparts (see next section), respectively. Small dots (red) correspond to sources with unreliable quantile values affected by poor photon statistics ( $\leq 10$ photons).

\section{Optical and near-IR analysis}

\subsection{Counterparts}

The large amounts of gas, dust, and selective extinctions of the region, combined with the absence of available deep $(V>$ $19 \mathrm{mag}$ ) optical photometry, prevent finding optical counterparts for most of our X-ray sources. We consider it appropriate to only use optical data to identify the massive star population (typical $V<12$, Massey \& Johnson 1993) of the region (i.e. Wolf-Rayet, $\mathrm{O}$ - and early B-types). We used a recent compendium of massive stars in the Carina Nebula presented by Smith (2006). Out of a total of 60 stars, 44 lie within the $0.0823 \mathrm{deg}^{2}$ FOV of our X-ray observation. We added the binary $(\mathrm{O} 5.5 \mathrm{~V}+\mathrm{O} 9.5 \mathrm{~V})$ FO 15 (Niemela et al. 2006), not included in the list of Smith (2006). Our final list of 45 hot massive stars is comprised of 1 LBV, 1 Wolf-Rayet, 21 O-type stars, and 22 early B-type stars. With a cross-identification radius of $3 \mathrm{arcsec}$, a total of $28 \mathrm{X}$-ray sources were identified. All the O-type stars were identified as X-ray emitters, while just 7/22 ( 31\%) of early B-type (SpT. between $\mathrm{B} 0 \mathrm{~V}$ to $\mathrm{B} 1.5 \mathrm{~V}$ ) have detectable $\mathrm{X}$-ray emission. The list of massive stars with X-ray counterparts and the discussion of their X-ray properties is presented in Sect. 8.

Apart from the massive stars, our X-ray population is essentially composed of low-mass stars. In such a young cluster as $\operatorname{Tr} 16$, low-mass stars are often optically invisible, being embedded and/or obscured by high amounts of gas and dust. We partially solve this problem by using the near-IR observations, on which the impact of dust extinction is reduced. We adopt $J(1.25 \mu \mathrm{m}), H(1.65 \mu \mathrm{m})$, and $K_{\mathrm{s}}(2.17 \mu \mathrm{m})$ photometry from the Two Micron All Sky Survey (2MASS) Point Source Catalog (PSC) ${ }^{16}$. The 2MASS is complete to magnitudes of 15.8, 15.1, and $14.3 \mathrm{mag}$ in the $J, H$, and $K_{\mathrm{s}}$ bands, respectively. We restricted our photometry analysis to 2MASS sources with flag quality $\mathrm{A}-\mathrm{C}$, or $\mathrm{D}$ in at least one of the three magnitudes (see

16 See http://www.ipac. caltech.edu/2mass explanation in the 2MASS All-Sky Data Release User's Guide). With this restriction, 20 sources were removed from our initial list of 5938 sources in the ACIS FOV of our observation. This leaves a total of 5918 2MASS objects ${ }^{17}$.

We began by cross identifying our X-ray source list with the 2MASS catalog. Identification radii, $R_{\text {id }}$, were chosen to limit the number of spurious identifications due to chance alignments, $N_{\text {chance }}$, and at the same time to include a large number of the true physical associations, $N_{\text {true }}$. Identification radii used were 1.0, 1.5, 2.1, and 2.7 arcsec adopted for the four different off-axis angle ranges: [0-2), [2-4), [4-7), and $>7$ arcmin, respectively ${ }^{18}$. Results of the final identification are presented in Sect. 4.3 and shown in Table 2. The first seven columns are: X-ray source number; 2MASS nomenclature for identified sources; the offset between the two positions; $J, H, K_{\mathrm{s}}$ magnitudes; photometry quality flag (Ph.Q); confusion flag (Cont). A total of $660 \mathrm{X}$-ray sources out of the 1035 in our list were identified with 665 2MASS objects. Five X-ray sources (\#96, \#382, \#401, \#816, and \#1034) were identified with two 2MASS counterparts each. After a visual inspection, we kept only the closer counterparts. Thus the final list of near-IR counterparts consists of 660 entries.

We estimate the expected number of extragalactic sources in our detection list by following the Flaccomio et al. (2006) procedure. We consider the ACIS count-rates of non-stellar sources in the Chandra Deep Field North (CDFN, Alexander et al. 2003; Barger et al. 2003) and estimate absorption corrected count-rates assuming $N_{\mathrm{H}}=5 \times 10^{21} \mathrm{~cm}^{-2}$ (from $A_{\mathrm{v}} \sim 3.6$, see Sect. 4.3) using PIMMS and assuming power-law spectra with index 1 and 2 (Giacconi et al. 2001). We then compare these count rates with upper limits taken at random positions in the ACIS FOV. For $\Gamma$ between 1 and 2 we obtain 72 to 95 expected extragalactic sources. Given the intrinsic near-IR fluxes of these sources and the absorption toward Trumpler 16, they are expected to be among the 385 without NIR counterparts (cf. Flaccomio et al. 2006). This means that no more than 18 to $24 \%$ of the unidentified X-ray population is related to extragalactic sources.

\subsection{Unidentified $X$-ray sources}

A large population of young stars, proto-stars, deeply embedded in dense circumstellar gas and dust should be present in the Carina Nebula (Smith et al. 2003). However optical and near-IR counterparts of young stellar objetcs (YSOs) are difficult to detect. Fortunately, X-ray emission is expected in YSOs along all their initial phases (Montmerle et al. 2000). X-ray photons easily escape from dense circumstellar material, where absorption process becomes important mainly for energies below $1.2 \mathrm{keV}$ (Morrison \& McCammon 1983b). This makes hard X-ray energies the most appropriate "window" to detect counterparts of deeply embedded young sources.

Of the $375 \mathrm{X}$-rays sources without near-IR counterparts, just a small fraction $(\sim 20 \%)$ is expected to be extra-galactic contamination (see Sect. 4.1). We are thus dealing with about $\sim 300$ candidate young (first stage) low-mass stars, highly obscured by circumstellar material. In our data these sources typically have lower X-ray photon statistics than those with a near-IR counterpart, i.e. $\sim 8$ vs. 28 average photons, respectively. In X-rays, no quantitative differences in the median energy and spectral

\footnotetext{
17 We note that the 2MASS catalog appears to have a "hole" around $\eta$ Carinae ( $\sim 1.5$ arcmin radius).

${ }_{18}$ The adopted radii were computed following technical procedures presented in Albacete Colombo et al. (2007).
} 
Table 2. Near-IR counterparts of Trumpler $16 \mathrm{X}$-ray sources. The complete version is available in the electronic version.

\begin{tabular}{|c|c|c|c|c|c|c|c|c|c|c|}
\hline$N_{x}$ & 2MASS J+ & Offset (") & $J$ mag & $H$ mag & $K_{\mathrm{s}} \mathrm{mag}$ & $\begin{array}{l}\text { Ph.Q } \\
\end{array}$ & Cont. & $A_{\mathrm{v}}$ & Mass & Notes \\
\hline 1 & $10433859-5938306$ & 2.33 & $14.57 \pm 0.03$ & $13.21 \pm 0.04$ & $12.63 \pm 0.03$ & AAA & 000 & 7.93 & 2.05 & \\
\hline 2 & -------- & -- & ----- & ----- & ----- & -- & -- & -- & -- & \\
\hline 3 & $10434145-5942245$ & 0.30 & $14.18 \pm 0.03$ & $13.20 \pm 0.02$ & $12.82 \pm 0.02$ & AAA & 000 & 4.59 & 2.24 & \\
\hline 4 & $10434126-5941002$ & 2.62 & $15.47 \pm--$ & $15.31 \pm 0.14$ & $14.60 \pm 0.14$ & UBB & 000 & -- & -- & \\
\hline 5 & $10434295-5944080$ & 1.77 & $15.63 \pm 0.08$ & $14.31 \pm 0.06$ & $13.27 \pm 0.04$ & AAA & 000 & 15.27 & 1.25 & $K$-excess \\
\hline 6 & -------- & -- & ----- & ----- & ----- & -- & -- & -- & -- & \\
\hline 7 & $10434401-5948177$ & 0.76 & $8.70 \pm 0.02$ & $8.51 \pm 0.04$ & $8.47 \pm 0.02$ & AAA & 000 & 1.38 & 19.2 & $09.5 \mathrm{~V}$ \\
\hline 8 & $10434538-5939468$ & 1.92 & $15.90 \pm--$ & $14.88 \pm--$ & $14.90 \pm 0.16$ & UUC & 000 & -- & -- & \\
\hline 9 & $10434536-5938471$ & 0.36 & $16.02 \pm 0.10$ & $14.04 \pm 0.04$ & $13.02 \pm 0.04$ & AAA & 000 & 14.90 & 1.01 & \\
\hline 10 & $10434525-5941567$ & 2.61 & $14.37 \pm 0.06$ & $12.99 \pm 0.05$ & $12.18 \pm 0.04$ & AAA & 000 & 12.05 & 2.16 & $K$-excess \\
\hline 11 & $10434659-5949292$ & 1.68 & $13.69 \pm 0.05$ & $12.68 \pm 0.05$ & $12.25 \pm 0.04$ & AEA & $\mathrm{c} 0 \mathrm{c}$ & -- & 2.38 & \\
\hline 12 & $10434809-5949246$ & 0.50 & $13.51 \pm--$ & $13.09 \pm 0.07$ & $12.85 \pm 0.05$ & UAA & $0 \mathrm{cc}$ & -- & -- & \\
\hline 13 & $10434937-5944549$ & 1.45 & $12.67 \pm 0.02$ & $12.20 \pm 0.03$ & $12.06 \pm 0.03$ & AAA & 000 & 0.82 & 4.89 & \\
\hline 14 & $10435007-5945530$ & 0.59 & $15.56 \pm 0.05$ & $14.59 \pm 0.03$ & $14.19 \pm 0.07$ & AAA & 000 & 4.31 & 1.30 & \\
\hline 15 & $10435085-5937437$ & 1.28 & $14.86 \pm 0.05$ & $13.74 \pm 0.05$ & $13.40 \pm 0.05$ & AAA & 000 & 3.76 & 1.86 & \\
\hline 16 & $10435088-5950307$ & 0.47 & $12.20 \pm 0.02$ & $11.99 \pm 0.03$ & $11.88 \pm 0.02$ & AAA & 000 & 0.28 & 6.22 & \\
\hline 17 & $10435123-5940243$ & 1.06 & $14.60 \pm 0.05$ & $13.38 \pm 0.04$ & $12.88 \pm 0.04$ & AAA & 000 & 6.57 & 2.03 & \\
\hline 18 & $10435132-5945239$ & 2.64 & $15.54 \pm 0.08$ & $14.06 \pm--$ & $13.55 \pm--$ & AUU & сpp & -- & 1.31 & \\
\hline 19 & $10435191-5940353$ & 0.40 & $16.49 \pm 0.16$ & $15.15 \pm 0.09$ & $14.63 \pm 0.11$ & CAA & 000 & -- & 0.70 & \\
\hline 20 & $10435186-5948017$ & 2.02 & $14.49 \pm 0.05$ & $13.46 \pm 0.06$ & $12.95 \pm 0.05$ & AAA & 000 & 6.60 & 2.09 & \\
\hline 21 & $10435223-5941574$ & 0.29 & $14.83 \pm 0.05$ & $14.46 \pm 0.08$ & $14.35 \pm 0.11$ & AAA & 000 & -- & 1.88 & \\
\hline 22 & $10435230-5939222$ & 1.87 & $13.03 \pm 0.04$ & $11.90 \pm 0.06$ & $11.20 \pm 0.04$ & AEE & 000 & -- & 3.55 & Mass-deg \\
\hline 23 & $10435408-5941463$ & 1.19 & $15.40 \pm 0.07$ & $14.24 \pm 0.05$ & $13.79 \pm 0.05$ & AAA & 000 & 5.47 & 1.40 & \\
\hline 24 & $10435419-5938073$ & 2.13 & $13.03 \pm--$ & $13.70 \pm 0.06$ & $13.53 \pm 0.07$ & UAA & $0 \mathrm{cc}$ & -- & -- & \\
\hline 25 & $10435501-5936242$ & 0.75 & $11.64 \pm 0.02$ & $11.48 \pm 0.03$ & $11.37 \pm 0.03$ & AAA & 000 & 0.42 & 8.28 & \\
\hline 26 & $10435505-5947505$ & 0.70 & $14.18 \pm 0.03$ & $13.81 \pm 0.04$ & $13.70 \pm 0.06$ & AAA & 000 & -- & 2.24 & \\
\hline 27 & $10435545-5942531$ & 0.50 & $14.93 \pm 0.05$ & $14.11 \pm 0.05$ & $13.84 \pm 0.06$ & AAA & 000 & 2.37 & 1.81 & \\
\hline 28 & $10435557-5949226$ & 0.39 & $13.44 \pm 0.03$ & $12.65 \pm 0.03$ & $12.24 \pm 0.03$ & AAA & 000 & 5.08 & 2.45 & \\
\hline 29 & $10435606-5949351$ & 1.97 & $14.53 \pm 0.05$ & $13.83 \pm 0.05$ & $13.60 \pm 0.06$ & AAA & 000 & 1.87 & 2.07 & \\
\hline 30 & $10435684-5942364$ & 0.45 & $14.70 \pm 0.05$ & $13.57 \pm 0.03$ & $12.88 \pm 0.04$ & AAA & 000 & 9.63 & 1.97 & $K$-excess \\
\hline
\end{tabular}

Column 3 ("Offset") is the offset between X-ray and near-IR counterpart. Ph.Q refers to the 2MASS photometric quality flags for the $J$, $H$ and, $K_{\mathrm{s}}$ bands: " $A$ " to " $D$ " indicate decreasing quality of the measurements, " $U$ " that the value is an upper limit. The next column refers to the contamination and confusion flag: for further analysis we considered only sources unaffected by known artifacts, i.e. Cont. = 000 (see $2 \mathrm{MASS}$ documentation for details). Masses are given in solar units, and the last column contains information presented in Sect. 4. Note: the "MASS-DEG." flag indicates mass degeneracy according to a Siess-based Jmag-Mass calibration.

quantiles were found for the X-ray sources with and without near-IR counterparts.

\subsection{Near-IR properties of identified X-ray sources}

We now investigate the near-IR properties of the X-ray sources. For this purpose we restrict our analysis to sources with highquality photometry $(\mathrm{Ph} . \mathrm{Q}=\mathrm{AAA})$ and no confusion $($ Cont. = 000). With these requirements the total number of IR sources in the ACIS FOV is reduced from 5918 to 2178 . We have also set a further requirement on near-IR counterparts of X-ray sources, that their $J, H$, and $K_{\mathrm{s}}$ magnitude errors be all $<0.1 \mathrm{mag}$. All these requirements yield $367 \mathrm{X}$-ray sources with good near-IR counterparts, out of the original 660.

Figure 3 shows the $J-H$ vs. $H-K_{\mathrm{s}}$ color-color (CC) diagram for these AAA-flagged sources. We also plot for comparison the MS (Kenyon \& Hartmann 1995), the classical T Tauri stars (CTTS) locus of Meyer et al. (1997), and reddening vectors starting from these loci and with slope $\left(A_{K \mathrm{~s}} / E\left(H-K_{\mathrm{s}}\right)=0.125\right)$ corresponding to the extinction law given by Hanson (2003). Trumpler 16 members with purely photospheric emission should lie in this reddening band. Otherwise, YSOs, such as CTTSs, and Herbig Ae/Be stars, because of the NIR excess emission originating in the inner parts of their circumstellar disks, are often found to the right of this band, i.e. in the CTTS locus. Fiftyone (out of 367) X-ray sources, i.e. likely Trumpler 16 members, have colors consistent with the (reddened) CTTS locus. This means a fraction of 51/339 (28 OB stars were discarded) $\sim 15 \%$ of all (low-mass) identified X-ray sources in the CC diagram. Of all 51 sources with intrinsic $K$-excess i.e. disk-star systems, eleven (Src-Id: 36, 41, 209, 230, 773, 966, 993, 996, 1002, 1003, and 1009) appear below the CTTS vectors, but with intrinsic bright $K_{\mathrm{s}}$ magnitudes, as is shown in both panels of Fig. 4. They are probably intermediate- to high-mass young stars with an intrinsic $K$-band excess that would be produced by massive accretion disks and/or extended envelopes surrounding massive YSOs. If confirmed, they will contribute about $40 \%$ of the total massive star population of the Trumpler 16 region.

Figure 4-left shows the $K_{\mathrm{s}}$ vs. $J-K_{\mathrm{s}}$ color magnitude (CM) diagram for the same stars plotted in Fig. 3. We also show for reference the expected cluster locus: the intrinsic $K_{\mathrm{s}}$ magnitudes and $J-K_{\mathrm{s}}$ colors for stars earlier than B5V were taken from the MS calibration of Knödlseder (2000) and Bessell \& Brett (1989). For later spectral types (masses between 0.1 and $7 M_{\odot}$ ), we adopted the 3 Myr isochrone from Siess et al. (2000), converted to the observational plane using the calibration given by Kenyon \& Hartmann (1995). The adopted MS and 3 Myr isochrone overlap satisfactorily.

In order to estimate the typical visual absorption of cluster members, we computed the distance of each X-ray source from the cluster locus along the reddening direction on the $K_{\mathrm{S}}$ vs. $J-H$ plane (see Fig. 4-right). Resulting $A_{\mathrm{v}}$ values for individual 


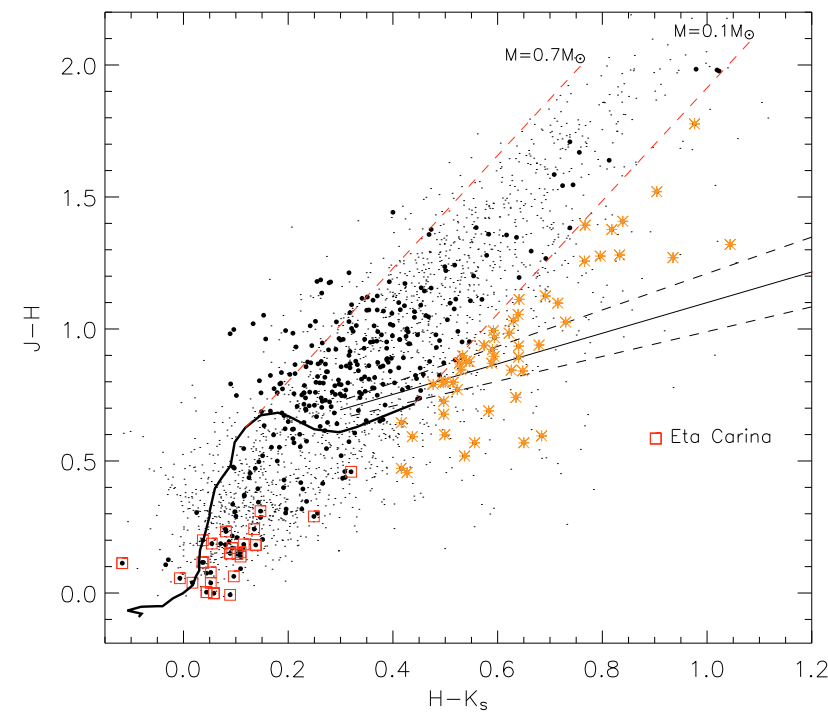

Fig. 3. $J H K_{\mathrm{S}}$ color-color diagram from high-quality $2 \mathrm{MASS}$ photometry. FILLED CIRCLES and DOTS refer to X-ray detected and undetected 2MASS sources, respectively. ASTERISKS show the source population with intrinsic $K_{\mathrm{s}}$ excess. Identified 2MASS source known as O- and early B-type stars are indicated by SQUARES. The main sequence is shown for reference. We also show the classical T Tauri stars (CTTS) locus of Meyer et al. (1997) and reddening vectors (dashed lines) with length corresponding to $A_{\mathrm{v}}=13$ mag. Note: $(i)$ the 2 MASS photometry of $\eta$ Carinae is severely affected, with Phot.Qual. = "DDD"; (ii) the peculiar position of the red box with $H-K_{\mathrm{s}}<-0.1$ corresponds to the massive binary CPD-592628AB (SpT: O9.5V+B0.3V).

sources $^{19}$ are listed in Col. 9 of Table 2. Note that for $11.2<$ $K_{\mathrm{S}}<12.8$ the absorption cannot be constrained because the reddening vector intersects the cluster locus more than once. In both CM diagrams, around $20 \mathrm{X}$-ray sources lie to the left of or relatively close to the reddened cluster loci. These are likely to be foreground MS stars, thus their $A_{\mathrm{v}}$ values, either cannot be computed or are close to zero. In a statistical sense, the median $A_{\mathrm{v}}$ value of OB stars $\left(A_{\mathrm{v}}=2.0 \pm 0.8 \mathrm{mag}\right)$ is lower than what is computed for low-mass stars $\left(A_{\mathrm{v}}=3.6 \pm 2.4 \mathrm{mag}\right.$, considered to be the typical absorption of the cluster). Obviously, the above estimates depend on the reliability of the assumed cluster locus and on the assumption that disk-induced excesses do not significantly affect the $J$ and $H$ magnitudes. As a caution against possible contamination and/or anomalous $J$ magnitude, but in particular for the $H$-band, the median $A_{\mathrm{v}}$ for the low-mass star population was estimated by discarding sources with intrinsic $K_{\mathrm{s}}$ excesses (i.e. labeled with asterisks in Figs. 3 and 4). We must note that: $(i)$ the dispersion along the $J-K_{\mathrm{s}}$ axis (see Fig. 4-left) indicates differential absorption of the region, and translates into a broad $A_{\mathrm{v}}$ distribution, with a spread $1 \sigma \sim 2.4 \mathrm{mag}$; and (ii) the difference between median $A_{\mathrm{v}}$ values of low-mass and OB stars suggests a clearing effect of strong winds and the radiation field of massive stars on their surrounding environment. This conclusion has also been reached by Albacete Colombo et al. (2007) for massive stars of the $\mathrm{Cyg} \mathrm{OB} 2$ region. However, compared to the Cyg OB2 region, the fraction of disk-stars members in the Trumpler 16 region is about four times greater.

Finally, we used the 2MASS $J$-band magnitudes to obtain an estimate of stellar masses for 510 counterparts (of a total 660 identified stars) with $J$-band Phot.Qual. = "A" to "D". We

\footnotetext{
19 Individual photometric errors of up to 0.1 mag at $K_{\mathrm{s}}$ and $J-H$ color errors of $\sim 0.14$, could results for $A_{\mathrm{v}}$ errors up to $\sim 0.7 \mathrm{mag}$.
}

computed the mass vs. $J$ mag relationship appropriate for the cluster mean age ( $3 \mathrm{Myr}$ ), distance ( $\mathrm{DM}=11.78 \mathrm{mag}$ ), and extinction $\left(A_{\mathrm{v}}=3.6 \mathrm{mag}\right)$, is with the last obtained as described above for the cluster locus in the CM diagrams ${ }^{20}$. We used Siess et al. (2000) models to compute PMS tracks of low- and intermediate-mass stars (masses $\leq 7 M_{\odot}$ ). We interpolated the $J$ mag - mass relation, using the $J$ magnitude vs. mass relationship at $3 \mathrm{Myr}$ (see Col. 10 of Table 2), to compute individual masses of stars ${ }^{21}$. Unfortunately, the relation mass- $J$ mag degenerates in the mass ranges $0.2-0.65 M_{\odot}$ (3 sources) and 2.7-4.52 $M_{\odot}$ (21 sources). We indicate these sources with "Mass-deg." flag in Col. 11 of Table 2, and give mean mass values for sources lying in these two ranges, i.e. 0.42 and 3.55 , respectively. Computed masses over $7 M_{\odot}$ are potentially affected by large uncertainties in the extrapolated $J$ mag - mass relation, and these values were excluded from further analysis. In Table 2 we give masses for a total of 510 stars, of which 410 range between 0.65 and $2.52 M_{\odot}$.

\section{X-ray variability}

PMS stars have high levels of X-ray activity that are commonly attributed to a "scaled up" solar-like corona formed by active regions. X-ray variability over a wide range of time scales is common in all magnetically active stars (e.g. Feigelson \& Montmerle 1999; Favata \& Micela 2003; Güdel 2004). On long time scales, this includes rotational modulation of active regions, their emergence and evolution, and magnetic cycles (e.g. Marino et al. 2003; Flaccomio et al. 2005). Most of the observed variations have short time scales ( $\sim$ hours), however, and can be attributed to small-scale flares triggered by magnetic reconnection events.

We first investigated X-ray variability in our sources using the non binned one-sample Kolmogorov-Smirnov (KS) test (Press et al. 1992). This test compares the distribution of photon arrival times with what is expected for a constant source. The test was applied to photons in the source's extraction regions, which also contain background photons. Given that the background was found to be constant with time (Sect. 2.1), the results, i.e. the confidence with which we can reject the hypothesis that the flux was constant during our observation, can be attributed to the source photons. Column 18 of Table 1, reports the logarithm of the KS-test significance with values $<-4$ truncated at that value: sources with $\log \left(P_{\mathrm{KS}}\right)<-3.0$ can be considered almost definitely variable because we expect at most one of the 1035 sources (i.e. $\leq 0.1 \%$ ) to be erroneously classified as variable. Seventy-seven X-ray sources $(\approx 7.4 \%$ of the total $)$ fall in this category. Fifty-five sources with $-2.0<\log \left(P_{\mathrm{KS}}\right)<-3.0$ can be considered as likely to be variable, although about half of them suffer from low photon statistics. These numbers of sources are lower limits to the total number of variable sources in the region for several reasons: (i) most of the observed variability is in the form of flares, i.e. events that are shorter than our observation and with a duty cycle that may be considerably longer (Wolk et al. 2005); (ii) the sensitivity of statistical tests to time variability of a given relative amplitude depends critically on photon

20 The choice of the $J$ band is justified because, (i) in the presence of disk excesses, the $J$-band is the most representative of the photospheric emission, i.e. least affected; and (ii) the mass ranges in which the massluminosity relationship is degenerate are narrower than for a similar relationship in the $H$ and $K_{\mathrm{s}}$ bands.

21 This method suffers from photometry inaccuracy, distance, and age spread. Mass values should be adopted carefully and should not be considered to measure the slope of the mass function. 

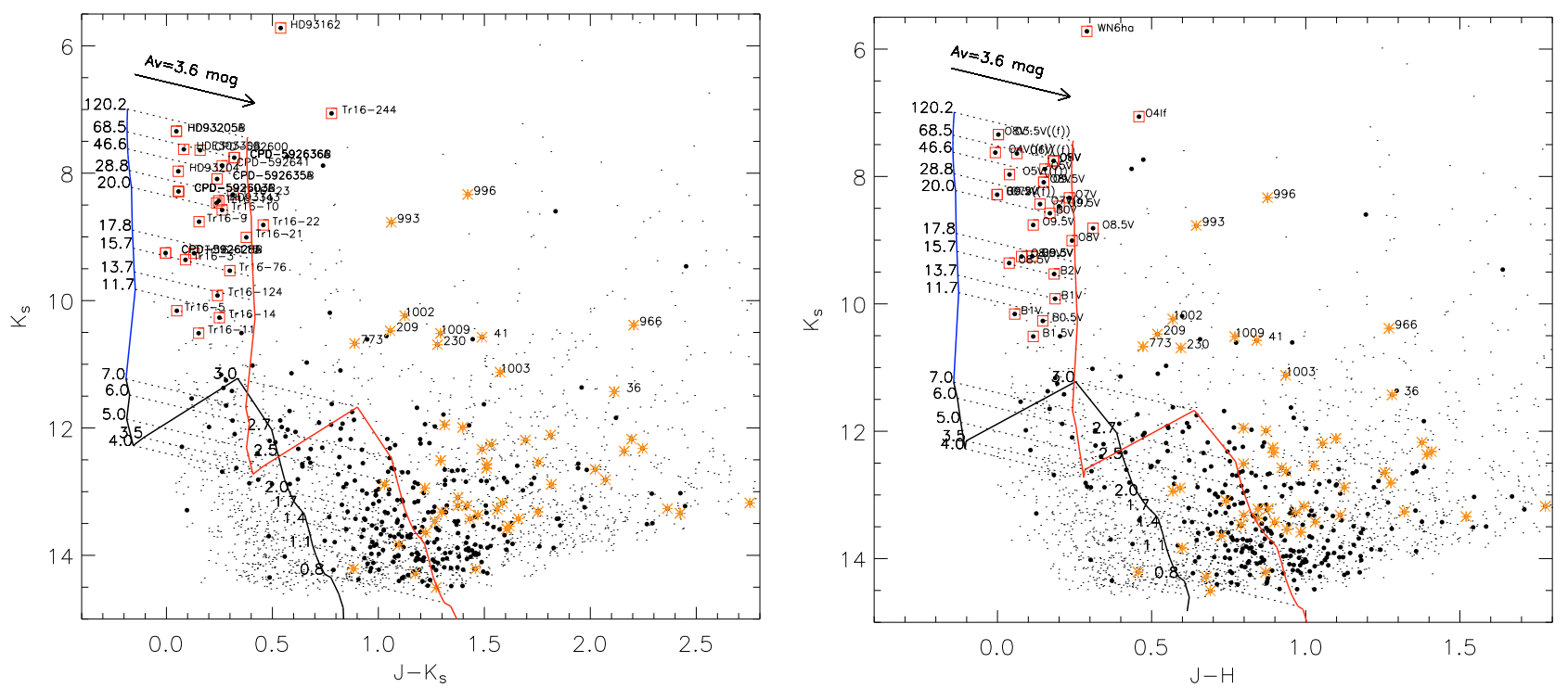

Fig. 4. CM diagrams of the Trumpler 16 region. Symbols as in Fig. 4. The two parallel curves indicate the expected cluster loci for the assumed distance and no reddening, and for the mean reddening $A_{\mathrm{v}}=3.6 \mathrm{mag}$. Masses are indicated on the left side of the unreddened isochrone. Identified OB stars show $K_{\mathrm{s}}$ magnitudes below 11 . Stars with $K_{\mathrm{s}}$ excess show typically $J-K_{\mathrm{s}}>1$. Note: the limitation imposed by the 2 MASS photometry $\left(K_{\mathrm{s}} \sim 14.5 \mathrm{mag}\right.$ ) corresponds to a mass limit of $1.1 M_{\odot}$. Labels near OB stars refer to Simbad names (left) and respective Spectral types (right). The fact that stars Tr16-244 (O4If) and HD 93129AB (WN6ha+O4f) appear above the $120 M_{\odot}$ track does not mean that they are more massive, since their evolutionary stages cannot be compared with a MS isochrone. $\eta$ Carinae was discarded because of the bad 2MASS photometry (Phot.qual. $=$ "DDD", see text). Sources labeled with numbers indicate stars (with masses above $12 M_{\odot}$ ) probably surrounded by disks.

statistics (see Albacete Colombo et al. 2007). Hereafter, we consider those 77 sources with $\log \left(P_{\mathrm{ks}}\right)<-3$ as variable.

To get a more accurate description of the detected variability, we extracted binned light-curves for each of the 77 variable sources in the region. We adopted a bin length of $900 \mathrm{~s}$, a compromise between bins that are long enough to reach a good signal-to-noise ratio per bin for most sources and short enough to resolve the decay phase of typical flares. Since the background of our observation is both low (negligible for many sources) and constant in time, we did not apply any background subtraction to the presented light curves. In Fig. 5 we show examples of the different behaviors among the light-curves of variable sources. Source \#254 like others (\#35, \#270, \#438, \#468, \#480, \#596, \#761, and \#975) experience "impulsive" flares with very quick rises and decay phases of only a few hours. Others (sources \#503, \#36, \#41, \#136, \#164, \#260, \#489, \#523, \#731, and \#811) show longer ( 2 to $10 \mathrm{~h}$ ) flares. In several instances a second impulsive event is visible during the exponential decay of a previous flare (e.g. sources \#36, \#52, \#87, \#196, \#564, $\# 600$, \#620, and \#623). The case of source \#696 is a combination of both variability types, with two consecutive flares. Other sources like \#714 and \#651 (e.g. \#27, \#251, \#271, \#793, \#839, \#890, \#904 and \#980) show light curves that, instead of showing typical flares, are characterized by slow continuous rises or decays that might be explained by rotational modulation of non-homogeneously distributed plasma (Flaccomio et al. 2005). Finally, light-curves, like those observed for sources \#524, \#71, and $\# 933$, seem to be related to a combination of flare like activity and rotational modulation.

\subsection{Variability in massive stars}

Because X-ray emission from O stars, which is believed to be unrelated to solar-like magnetic activity, comes from the integrated emission from many small shocks randomly occurring in their strong winds (Feldmeier et al. 1997; Owocki \& Cohen 1999), on average, global X-ray variability is not expected to occur. However, it is surprising that three (out of 28) massive stars, namely Tr 16-11 (B1.5 V, source \#136), Tr 16-5 (B1 V, source \#489), and the binary HD 93205 (O3.5V((f))+O8V, source \#242), are significantly variable, with $\log \left(P_{\mathrm{KS}}\right)$ values lower than -3 . The origin of the observed flare-like variability in the first two sources (B-type stars) is probably coronal activity of unresolved late-type companions. This hypothesis was also proposed for Tr16-11 itself by Evans et al. (2003).

Figure 6 shows the X-ray light-curve of the HD 93205 binary system. Two different processes may be acting simultaneously to explain the observed variability:

- (i) The decrement of count rate agrees with phase-locked X-ray variability reported by Morrell et al. (2001). The X-ray count rate decreases from $\sim 0.055$ to $0.033 \mathrm{cts} / \mathrm{s}$ (about $35 \%$ ) in about $88 \mathrm{ks}$ ( $\sim 1$ day) of continuous observation, i.e. about $16 \%$ of the orbital period. This agrees with X-ray emission from colliding winds contributing most of the total detected emission. However, we cannot discard that magnetically channeled wind streams, at the $\mathrm{O} 3.5 \mathrm{~V}((\mathrm{f}))$ primary, colliding with the cool and dense postshock plasma at the magnetic equator (e.g. $\theta$ Orionis-C, Gagné et al. 2005a). A detailed spectral and time (phase-resolved) study should follow to discern between these two possibilities.

- (ii) The observed short time X-ray variability $\left(\log \left(P_{\mathrm{KS}}\right)=\right.$ -3.07). While dynamical instabilities (Luo et al. 1990) $)^{22}$ in the colliding wind region (CWR) seems to be more

22 Whenever the wind velocities of two stars are not equal, shocked CWR should be subject to the rapid growth of dynamical (Kelvin-Helmholtz) instabilities, reaching the radiative cooling state. This limit becomes important for massive binaries with typical orbital periods $\leq 20$ days (Antokhin et al. 2004). 

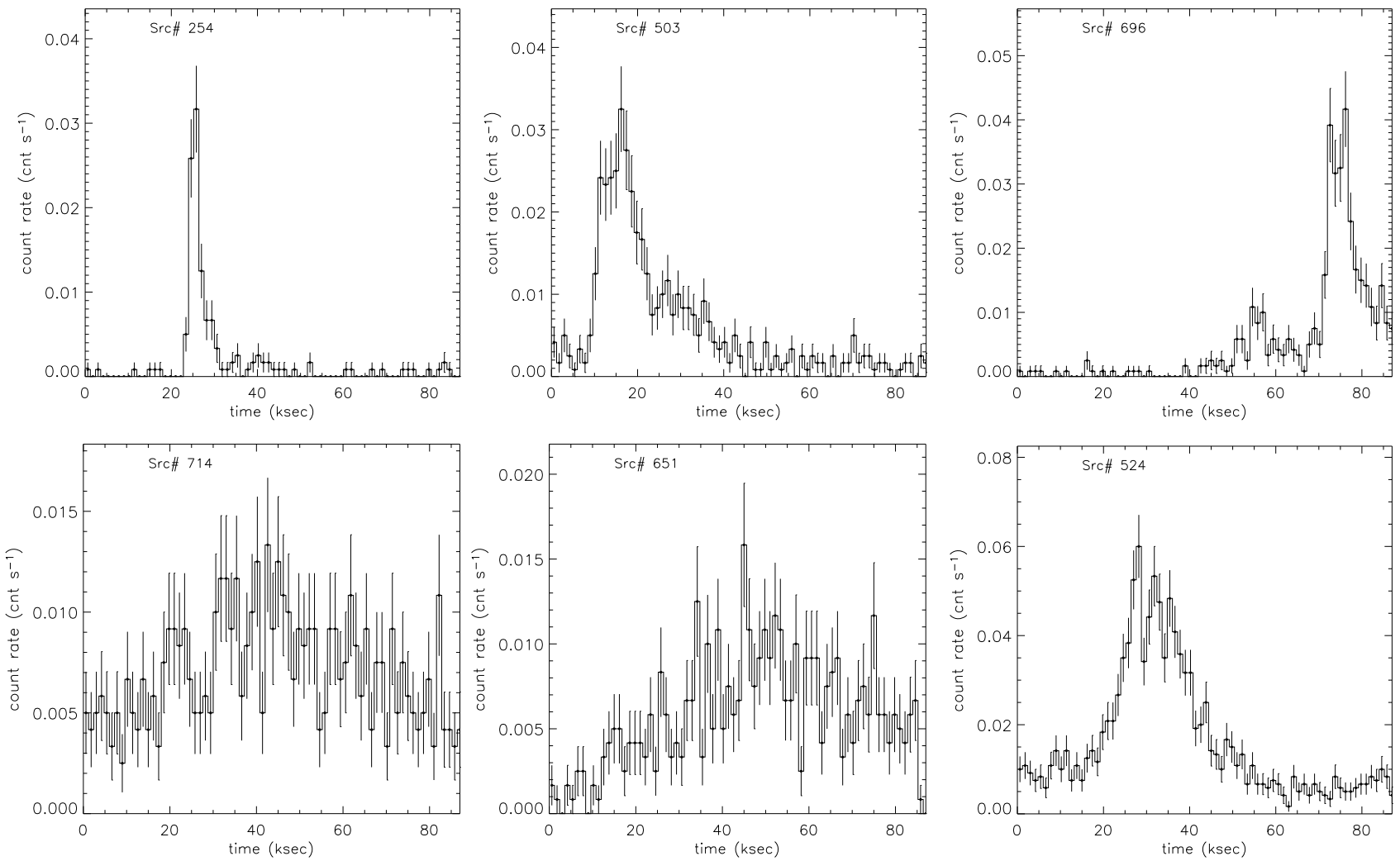

Fig. 5. Light curves (in the $0.5-8.0 \mathrm{keV}$ band) showing different variability scenarios occurring among our 77 variable sources. Bin size is $900 \mathrm{~s}$. The source number is indicated in each panel. Upper panels show a flare-like behavior with very quick rises and decay phases of hours, while bottom panels are slowly modulated variability. A probable flare+modulation variability is occurring in the last panel.

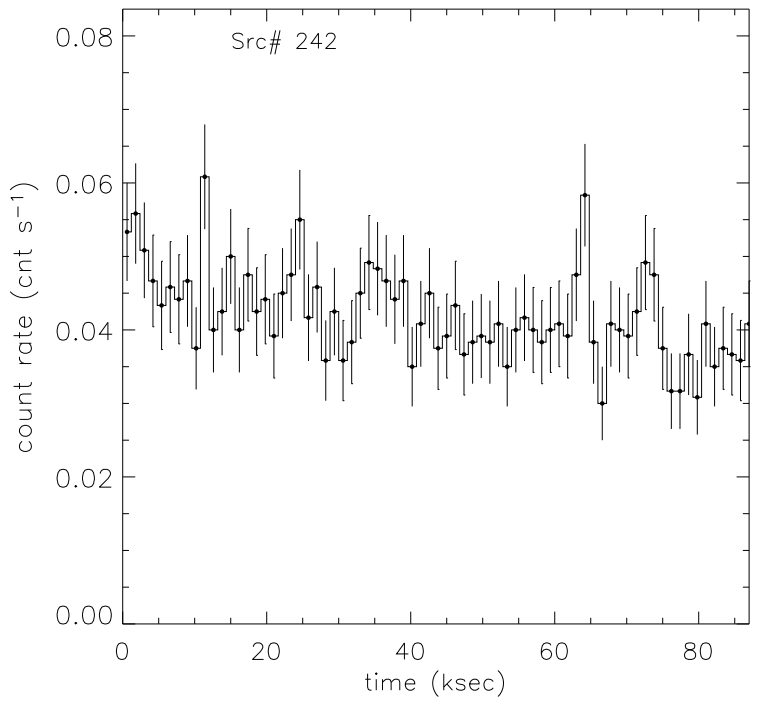

Fig. 6. X-ray light-curve of the massive binary $(\mathrm{O} 3.5 \mathrm{~V}((\mathrm{f}))+\mathrm{O} 8 \mathrm{~V})$ HD 93205. This light curve shows: $i-$ a uniform decay probably related to the orbital motion of the system, with changing absorption $N_{\mathrm{H}}$ of the colliding-wind region along the line of sight; plus $i i-$ a probable short-term variability (see text).

appropiate, the magnetic reconnection from an unknown low-mass tertiary companion ${ }^{23}$ cannot be ruled out.

23 Variability like HD 93205's has been seen also on theta 2 Ori-A and has been interpreted by Schulz et al. (2006) as evidence of binaryinduced reconnection. However, image reconstruction shows that hard,
We note that the origin of X-ray variability in high mass stars is beyond of the scope of this work, because it needs a larger X-ray data set. We leave this subject to a further investigation.

\section{Spectral analysis}

To characterize the hot plasma responsible for the X-ray emission of Trumpler 16 stars and to estimate their intrinsic X-ray luminosities, we analyzed the ACIS spectra of the 615 (out of all 1035) sources with more than 20 net photons (NET_CNTS), i.e. corrected for local background. Spectral parameters for sources with less than 20 net photons are much too ill-constrained (Albacete Colombo et al. 2007), and thus were not determined. Moreover, high local background could affect the reliability of computed spectral parameters. We defined the fraction $f_{\text {cont }}$ as the ratio between BKG_CNTS (number of background counts in the source extraction region) and NET_CNTS. We accepted spectral fits for sources with $f_{\text {cont }}<1$ and NET_CNTS $\geq 20$ photons. Of all 1035 sources, only 563 satisfy both conditions above, while 119 show $f_{\text {cont }}<1$, and 353 lie in the low statistics regime (i.e. NET_CNTS $<20 \mathrm{ph}$ ).

Source and background spectra in the $0.5-8.0 \mathrm{keV}$ band were produced with AE (see Sect. 3.2), along with individual "redistribution matrices files" (RMF) and "ancillary response files" (ARF). For model-fitting, spectra were grouped to have a specified number of events in each energy bin. Grouping was tuned to the source statistics, and we chose $2,5,7,10$, and 60 counts per channel for sources with net-counts in the following ranges: [20-40], [40-100], [100-200], [200-500],

short-term variations are from an intermediate-mass tertiary companion at $\sim 0.3 \operatorname{arcsec}$ (Harvin et al. 2002). 
Table 3. X-ray spectral fits of Trumpler 16 sources. The complete version is available in the electronic version.

\begin{tabular}{|c|c|c|c|c|c|c|}
\hline $\begin{array}{l}N_{x} \\
\#\end{array}$ & $\begin{array}{l}\text { Cnts. } \\
\text { (ph) }\end{array}$ & $\begin{array}{l}\text { Stat. } \\
\left(\chi_{v}^{2}\right)\end{array}$ & $\begin{array}{l}\log \left(N_{\mathrm{H}}\right) \\
\left(\mathrm{cm}^{-2}\right)\end{array}$ & $\begin{array}{l}k T \\
(\mathrm{keV})\end{array}$ & $\begin{array}{l}\log \left(L_{\mathrm{x}}\right) \\
(\mathrm{erg} / \mathrm{s})\end{array}$ & flag \\
\hline 1 & 30 & $\frac{1 v}{--}$ & ----- & ----- & 30.51 & no-fit \\
\hline 2 & 36 & -- & ----- & ----- & 30.58 & no-fit \\
\hline 3 & 208 & 0.66 & $22.01 \pm 0.16$ & $2.07 \pm 0.56$ & 31.58 & fitted \\
\hline 4 & 55 & 0.94 & $21.90 \pm 0.31$ & $2.96 \pm 2.61$ & 30.50 & fitted \\
\hline 5 & 28 & -- & ----- & ----- & 30.48 & no-fit \\
\hline 6 & 41 & -- & ----- & ----- & 30.64 & no-fit \\
\hline 7 & 47 & -- & ----- & ----- & 31.29 & $\operatorname{Tr} 16-19$ \\
\hline 8 & 54 & -- & ----- & ----- & 30.76 & no-fit \\
\hline 9 & 45 & -- & ----- & ----- & 30.68 & no-fit \\
\hline 10 & 25 & -- & ----- & ----- & 30.42 & no-fit \\
\hline 11 & 41 & -- & ----- & ----- & 30.72 & no-fit \\
\hline 12 & 132 & 1.50 & $21.23 \pm 0.62$ & $2.65 \pm 0.94$ & 31.08 & fitted \\
\hline 13 & 89 & 0.40 & $21.64 \pm 0.14$ & $0.67 \pm 0.93$ & 30.83 & fitted \\
\hline 14 & 15 & -- & ----- & ----- & 30.23 & no-fit \\
\hline 15 & 41 & -- & ----- & ----- & 30.65 & no-fit \\
\hline 16 & 23 & -- & ----- & ----- & 30.40 & no-fit \\
\hline 17 & 28 & -- & ----- & ----- & 30.48 & no-fit \\
\hline 18 & 31 & 0.80 & $22.32 \pm 0.32$ & $1.36 \pm 0.88$ & 31.04 & fitted \\
\hline 19 & 7 & -- & ----- & ----- & 29.93 & no-fit \\
\hline 20 & 30 & -- & ----- & ----- & 30.51 & no-fit \\
\hline 21 & 25 & 1.21 & $21.97 \pm 0.65$ & $0.29 \pm 0.18$ & 30.02 & fitted \\
\hline 22 & 176 & 0.94 & $21.81 \pm 0.22$ & $3.32 \pm 1.45$ & 31.46 & fitted \\
\hline 23 & 25 & -- & ----- & ----- & 30.43 & no-fit \\
\hline 24 & 22 & -- & ----- & ----- & 30.38 & no-fit \\
\hline 25 & 177 & 1.87 & $22.00 \pm 0.18$ & $2.17 \pm 0.74$ & 31.52 & fitted \\
\hline 26 & 44 & 0.90 & $21.70 \pm 0.27$ & $1.49 \pm 0.54$ & 30.38 & fitted \\
\hline 27 & 51 & 0.50 & $22.06 \pm 0.32$ & $2.11 \pm 1.27$ & 31.03 & fitted \\
\hline 28 & 77 & 0.47 & $21.28 \pm 0.72$ & $1.49 \pm 0.44$ & 30.83 & fitted \\
\hline 29 & 36 & -- & ----- & ----- & 30.59 & no-fit \\
\hline 30 & 12 & -- & ----- & ----- & 30.12 & no-fit \\
\hline
\end{tabular}

Notes: last column flag: sources with no spectral information (NO-FIT) have their X-ray luminosities computed by using an average count-rate to $L_{\mathrm{x}}$ conversion factor (see Sect. 7). "HARD TAIL" flag refers to the need of more components in the spectral models. Spectral fit parameters for OB-type stars are not listed here as they are presented in Table 4.

and [500-**]. Spectral fitting of background-subtracted spectra was performed with XSPEC v12.0 (Arnaud 2004) and our own shell and TCL scripts to automate the process as described in Flaccomio et al. (2006). Because background-corrected spectra are not appropriately handled by C-statistics (Getman et al. 2005), best-fit parameters for the chosen models were computed by chi-squared $\left(\chi^{2}\right)$ minimization.

We fit our spectra by assuming emission by a thermal plasma, in collisional ionization equilibrium, as modeled by the APEC code (Smith et al. 2001). Elemental abundances are not easily constrained with low-statistics spectra and were fixed at $Z=0.3 Z_{\odot}$, with solar abundance ratios taken from Anders \& Grevesse (1989). The choice of sub-solar abundances is suggested by several X-ray studies of star-forming regions (e.g. Feigelson et al. 2002; Preibisch 2003). Absorption was accounted for using the WABS model, parameterized by the hydrogen column density, $N_{\mathrm{H}}$ (Morrison \& McCammon 1983a). In Table 3 we give best-fit parameters $\left(\chi_{v}^{2}, N_{\mathrm{H}}, k T\right.$ and $\left.L_{\mathrm{x}}\right)$ of the sources.

Except for 28 massive O- and early B-type stars, we fit source spectra with one-temperature $(1 T)$ plasma models using an automated procedure. To reduce the risk of finding a local minimum in the $\chi^{2}$ spaces, our procedure chooses the best fit among several obtained starting from a grid of initial values of the model parameters: $\log \left(N_{\mathrm{H}}\right)=21.0,21.7,22.0,22.4,22.7$ and $23.0 \mathrm{~cm}^{-2}$ and $k T=0.5,0.75,1.0,2.0,5.0 \mathrm{keV}$. The bestfit values of $\log \left(N_{\mathrm{H}}\right)<20.3 \mathrm{~cm}^{-2}$ were truncated at 20.3 for two cases (sources \#150 and \#944) because, in the $0.5-8.0 \mathrm{keV}$ energy range, ACIS spectra are insensitive to lower column densities. In a similar way, above $10 \mathrm{keV}$ Chandra is not able to distinguish between such high temperatures. Therefore, 74 bestfit values of $k T$ above $8 \mathrm{keV}$ were truncated to that value. They are indicated with a flag HARD-TAIL in Table 3.

Figure 7 shows the distribution of best-fit $\log \left(N_{\mathrm{H}}\right)$ values for the 563 fitted sources. They appear to be normally distributed with a median $\log N_{\mathrm{H}} \sim 21.73\left(N_{\mathrm{H}}=5.37 \times 10^{21} \mathrm{~cm}^{-2}\right)$ and an FWHM of $\sim 0.4$ dex. The log-normal distribution of the sources is indicated with the Gaussian curve ${ }^{24}$. The computed median of $N_{\mathrm{H}}\left(\sim 5.37 \times 10^{21} \mathrm{~cm}^{-2}\right)$ is converted to a median $A_{\mathrm{v}}=3.35$ by use of the Vuong et al. (2003) relation: $N_{\mathrm{H}} / A_{\mathrm{v}}=$ $1.6 \times 10^{21}$ atoms cm$~^{-2} \mathrm{mag}^{-1}$. We also tested the relation $A_{\mathrm{v}}=$ $0.56 N_{\mathrm{H}}+0.23\left[N_{\mathrm{H}}\right.$ in $\left.10^{21}\right]$ (Predehl \& Schmitt 1995), for which the median $A_{\mathrm{V}}$ is $3.23 \mathrm{mag}$. Both of these values are in good agreement with the median $A_{\mathrm{v}}=3.6$ mag computed from our near-IR analysis (see Sect. 4.3). The $1 \sigma$ dispersion of the $N_{\mathrm{H}}$ distribution is 0.4 dex. It is translated into typical a $A_{\mathrm{v}}$ range between 1.3 to $6.7 \mathrm{mag}$ of visual extinction. The X-ray sources without near-IR counterparts seem to be distributed towards higher absorption values (median $\log \left(N_{\mathrm{H}}\right) \sim 21.9 \mathrm{~cm}^{-2}$ ) with respect to those with near-IR counterparts. Unfortunately, they generally have poor X-ray photon statistics and consequently a less reliable estimation of their X-ray spectral parameters.

Unlike the $N_{\mathrm{H}}$ distribution, the $k T$ distribution of plasma temperatures is not log-normal. It peaks at $\sim 1.6 \mathrm{keV}$, has a median $\sim 1.95 \mathrm{keV}$, and shows an extended hard tail attributed both to variable sources $\left(\log P_{\mathrm{KS}}<-3\right)$ with harder spectra (median $k T=3.25 \mathrm{keV}$, as expected from coronal heating processes involved in flare-like activity) and to highly absorbed sources (i.e. $A_{\mathrm{v}} \geq 6 \mathrm{mag}$ ) showing a median $k T \sim 2.6 \mathrm{keV}$ (while those in the range $1 \leq A_{\mathrm{v}} \leq 6 \mathrm{mag}$ are softer and distributed with a median $k T=1.75 \mathrm{keV})$.

Finally, suspected single massive stars show typically soft spectra with median $k T=0.62 \mathrm{keV}$, while for known massive binaries, this value rises to $k T=2.1 \mathrm{keV}$, no doubt due to hard X-ray photons being produced in the colliding wind region (CWR) of the massive O+OB binaries. Details of X-ray spectral characteristics of massive stars in the region are presented in Sect. 8.

\section{X-ray luminosity of stars}

Unabsorbed X-ray luminosities were computed for those sources with available spectral fits for the $[0.5-8.0] \mathrm{keV}$ energy range. For sources with no available and/or reliable spectral fit $(119+353$ out of 1035$) L_{\mathrm{x}}$ were computed using a single count rate to $L_{X}$ conversion factor $(\mathrm{CF})^{25}$. CF in Trumpler 16 is $8.3_{5.2}^{10.3} \times 10^{33} \mathrm{erg} / \mathrm{ph}$. Upper and lower values correspond to \pm $1 \sigma$ uncertainties, respectively.

In Fig. 8 we show the X-ray luminosity distribution for lowmass $^{26}$ stars, while known OB stars are plotted separately in

\footnotetext{
24 Seven sources (\#150, \#305, \#342, \#388, \#692, \#868 and \#944) appear to have $N_{\mathrm{H}}$ below $10^{21} \mathrm{~cm}^{-2}$, and are likely foreground stars.

${ }^{25}$ It was computed as the median ratio between the individual unabsorbed X-ray luminosities (from best-fit spectral models) and the source count rates.

${ }^{26}$ We defined the low-mass range $M<7 M_{\odot}$, based on the presence of a significant convection envelope (at the adopted age) that explains $\mathrm{X}$-ray emission from magnetic activity.
} 

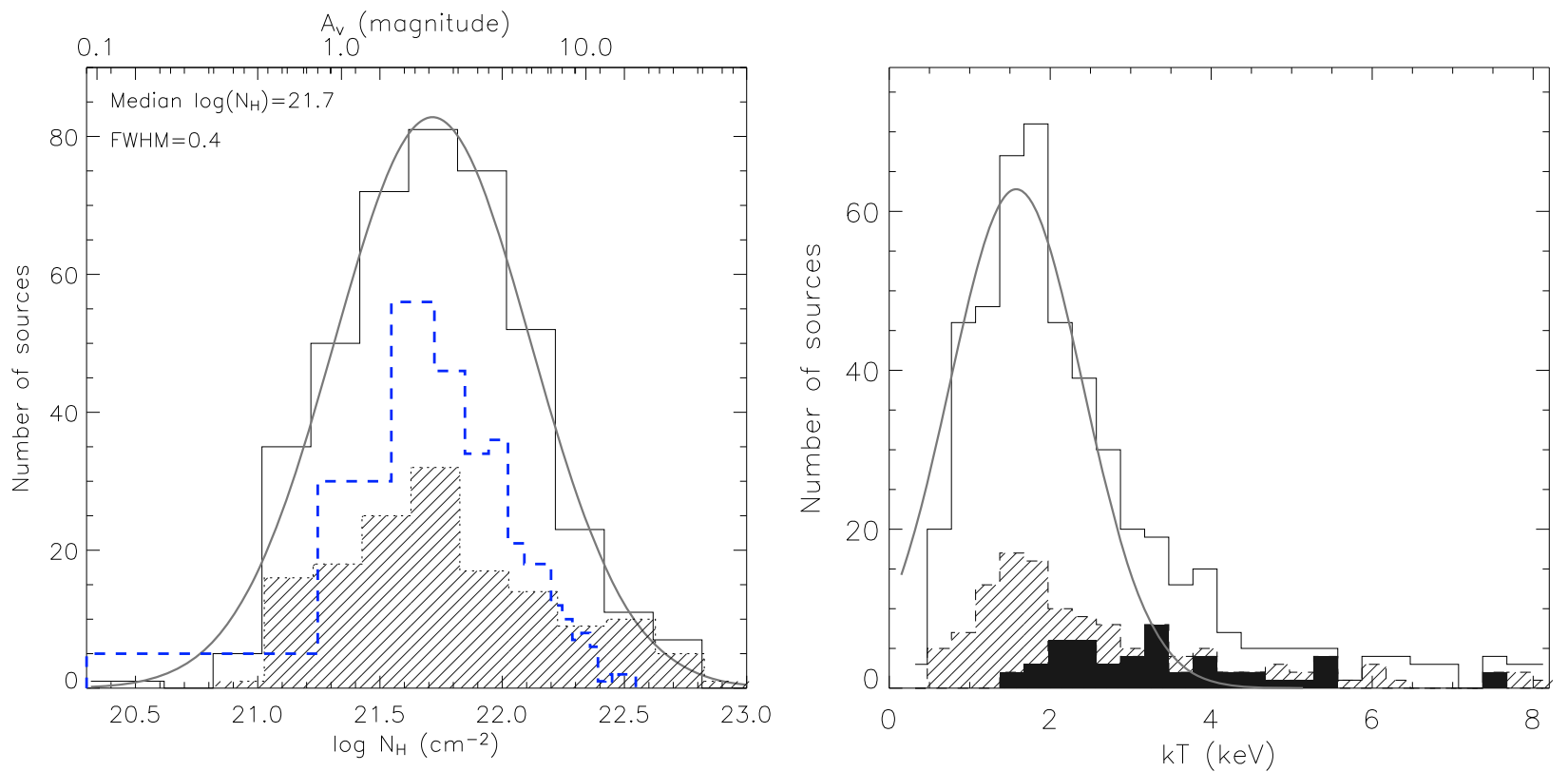

Fig. 7. Left: the solid histogram shows the $N_{\mathrm{H}}\left(A_{\mathrm{v}}^{\mathrm{xspec}}\right)$ distribution of 415 spectral fitted sources with $2 \mathrm{MASS}$ counterparts. The upper scale was converted using an $N_{\mathrm{H}} / A_{\mathrm{v}}$ ratio of $1.6 \times 10^{21}$. The thick dashed histogram shows the $A_{\mathrm{v}}^{\text {near-IR }}$ mag distribution for all near-IR counterparts as was computed in Sect. 4. Both distributions peak at $A_{\mathrm{v}}^{\mathrm{xspec}}=3.35(1 \sigma=0.4) \mathrm{mag}$ and $A_{\mathrm{v}}^{\text {near }-\mathrm{IR}}=3.26 \mathrm{mag}(1 \sigma=0.3$ dex $)$. The shadowed histogram shows the $N_{\mathrm{H}}$ distribution of 148 sources with no near-IR counterpart. Right: same as the left panel for plasma temperatures $(k T)$ with, in addition, the distribution for "flaring" sources (black-filled histogram). The peak of the overal distribution is at $\sim 1.6 \mathrm{keV}$.

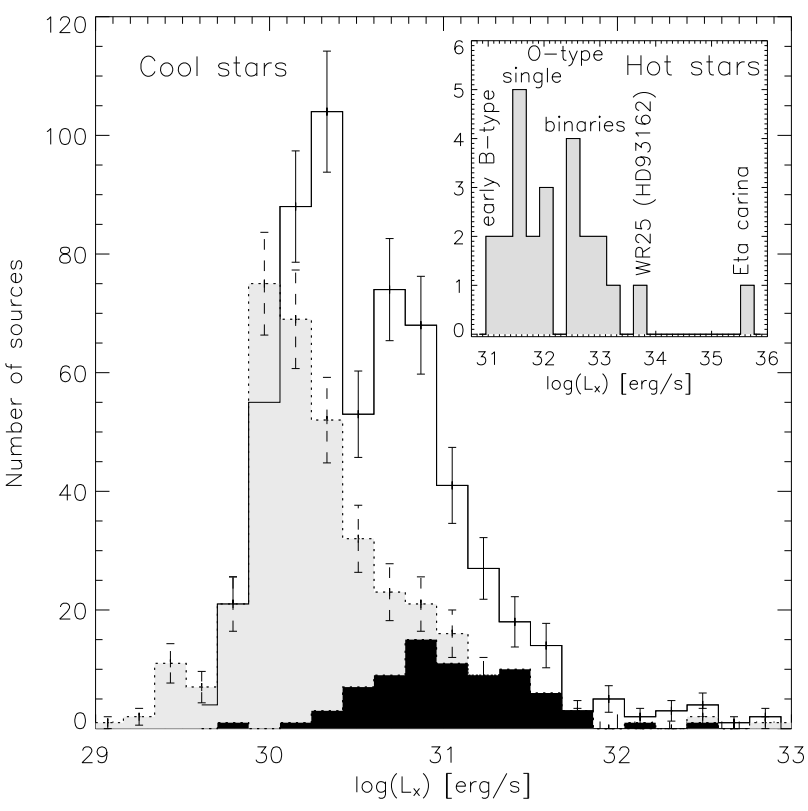

Fig. 8. The X-ray luminosity distribution for 592 low-mass stars computed from a single $L_{\mathrm{X}}$ count-rate conversion factor is shown as a solid line. The grey filled histogram shows the $L_{\mathrm{x}}$ distribution for $354 \mathrm{X}$-ray sources without near-IR counterpart. The 77 variable sources are shown with the black-filled histogram. Upper-right inset (Hot stars) shows the unabsorbed $L_{\mathrm{x}}$ from X-ray spectral fits (see Sect. 8). Error bars are $1 \sigma$ Poisson errors.

the upper inset histogram. The $L_{\mathrm{x}}$ distribution of sources has been plotted separately for (i) 592 X-ray sources with nearIR counterpart, not including $28 \mathrm{OB}$ stars and variable sources with a median $\log \left(L_{\mathrm{x}}\right) \sim 30.5 \mathrm{erg} \mathrm{s}^{-1}$. The peak of the distribution indeed marks the completeness limit of our X-ray observation. (ii) There are 354 unidentified sources, except for variable ones, which appear systematically less luminous than those with a 2 MASS counterpart, with median $\log \left(L_{\mathrm{x}}\right) \sim 30.2 \mathrm{erg} \mathrm{s}^{-1}$. (iii) Seventy seven variable sources, showing a median $\log \left(L_{\mathrm{X}}\right) \sim$ $31.1 \mathrm{erg} \mathrm{s}^{-1}$, i.e. about 4 times higher than the observed $L_{\mathrm{x}}$ for similar stars in a quiescent (non-flaring) phase. The upper inset in Fig. 8 shows the unabsorbed $L_{\mathrm{x}}$ for 28 massive stars (including 13 binary systems) in the region. By the following results and discussion presented in Sect. 8, binaries appear with typical $L_{\mathrm{x}}$ over $10^{+32} \mathrm{erg} \mathrm{s}^{-1}$, higher than observed for those suspected single B-type stars. As we discuss in Sect. 8, massive binaries have typical X-ray luminosities $L_{\mathrm{x}} \geq 10^{+32} \mathrm{erg} \mathrm{s}^{-1}$, higher than those of single OB stars.

We examined how X-ray activity depends on stellar mass for Trumpler 16 low-mass stars, and compares the results with those already known for the ONC and Cyg OB2 SFRs. To increase statistics, we used $L_{\mathrm{x}}$ values computed by means of the average CF. While $L_{\mathrm{x}}$ and mass for Cyg OB2 stars were computed following the same procedures used here (Albacete Colombo et al. 2007), for ONC stars they have been computed differently in the literature ${ }^{27}$. For the sake of homogeneity, we recomputed $L_{\mathrm{x}}$ and masses of ONC stars by using a single count rate to $L_{\mathrm{x}}$ conversion factor $\left(\mathrm{CF}_{\mathrm{onc}}=7.52 \times 10^{+32} \mathrm{erg} / \mathrm{ph}\right)$ and 2MASS photometry, respectively.

In Fig. 9 we show a plot of $L_{\mathrm{x}}$ vs. star mass. We used circles for all 510 Tr16 sources with estimated masses. Filled (329) and open (181) circles indicate $L_{\mathrm{x}}$ values computed from spectral fits and using the CF, respectively. We performed a linear regression for all sources in the 0.7-2.5 $M_{\odot}$ range: $\log \left(L_{\mathrm{x}}\right)=$ $30.26( \pm 0.11)+1.0( \pm 0.09) \log \left(M / M_{\odot}\right)$ with a standard deviation in the residuals of 0.38 dex. The power-law slope we find here agrees with the one found by Albacete Colombo et al. (2007) for the Cyg OB2 region: $\log \left(L_{\mathrm{x}}\right)=30.33( \pm 0.16)+$ $0.71( \pm 0.13) \log \left(M / M_{\odot}\right)$ for masses in the $0.5-3.0 M_{\odot}$ range.

27 In the ONC, stellar masses were computed from optical spectra, available for many stars, while $L_{\mathrm{x}}$ was obtained for many stars from an X-ray spectral analysis based on a relatively high photon statistics (Getman et al. 2005). 


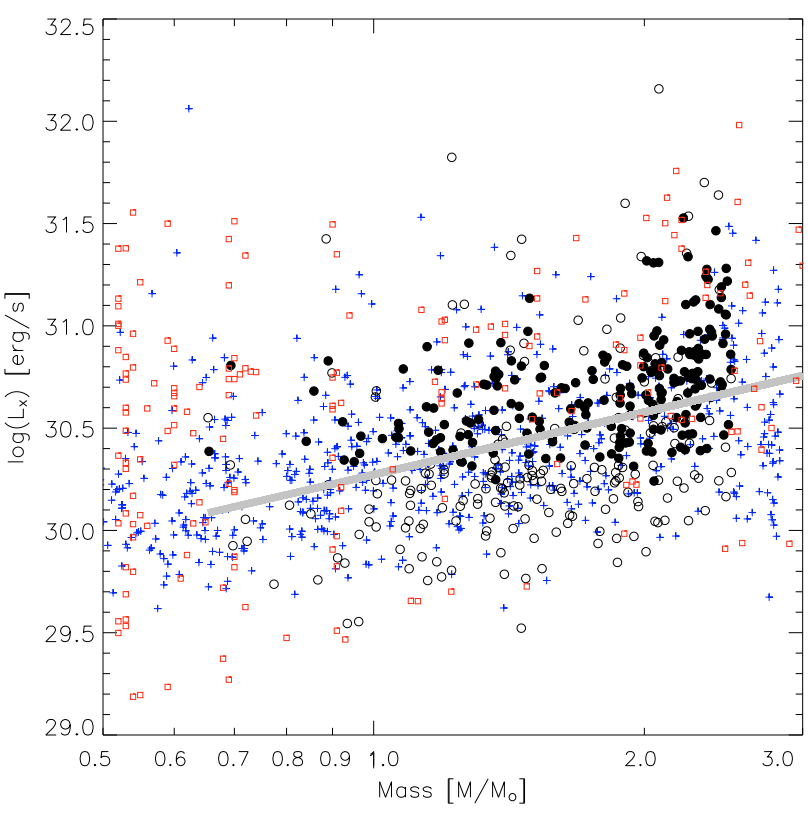

Fig. 9. X-ray luminosity vs. stellar mass for stars in the near-IR sample with masses determined from the PMS models of Siess et al. (2000). Tr16 stars are plotted by open and filled circles, corresponding to $L_{\mathrm{x}}$ from $\mathrm{CF}$ and spectral fits, respectively. Crosses indicate Cyg OB2 sources (Albacete Colombo et al. 2007), while small boxes are ONC sources (Preibisch et al. 2005). The thick gray line shows the linear regression fit to the $\operatorname{Tr} 16$ low-mass $\left(0.7-2.5 M_{\odot}\right)$ stars.

This slope also agrees with what we find for the ONC stars in the mass range $0.5-3.0 M_{\odot}$, namely $0.82 \pm 0.09$.

Changes in the X-ray activity of stars with different ages of the SFRs has been previously reported by Preibisch \& Feigelson (2005). To address this issue, we computed $L_{\mathrm{x}}$ detection limits for low-mass stars in the $\operatorname{Tr} 16$ (3 Myr), CygOB2 (2 Myr), and ONC (1 Myr) observations, as $\log \left(L_{\mathrm{x}}\right) \sim 30.5,30.3$ and $28.5 \mathrm{erg} \mathrm{s}^{-1}$, respectively, above which a source is detectable anywhere in the FOV. A mass-dependent completeness fraction $\left(f_{\text {comp }}\right)$ for our survey of Trumpler 16 stars was then computed by adopting the X-ray luminosity functions given by Preibisch \& Feigelson (2005): $f_{\text {comp }}$ is $\sim 5 \%$ for $0.1-0.5 M_{\odot}$, $\sim 40 \%$ for $0.5-0.9 M_{\odot}$, and $\sim 55 \%$ in the $0.9-1.2 M_{\odot}$ range. Our survey of $\operatorname{Tr} 16$ is statistically complete for masses $\geq 1.5 M_{\odot}$. In Fig. 10 we present $L_{\mathrm{x}}$ cumulative distributions for Trumpler 16, Cyg OB2, and ONC stars in the mass range 1.5-2.5 $M_{\odot}$ where all three data sets are complete. While Trumpler 16 and Cyg OB2 show very similar distributions, the ONC looks quite different from to $\operatorname{Tr} 16$. The distance between two distributions $(D=0.31)$ was computed using a two-sample Kolmogorov-Smirnov test (Press et al. 1992). We are able to confirm, with a probability $\geq 99.9 \%\left(\log \left(P_{\mathrm{ks}}\right)=-4.15\right)$, that young ONC (1 Myr) stars, with masses in the range $1.5-2.5 M_{\odot}$, are intrinsically more luminous in X-rays than their $\operatorname{Tr} 16$ (3 Myr) counterparts.

\section{X-rays from massive stars}

A variety of different physical mechanisms are responsible for the observed X-ray emission in OB-type stars. The most widely accepted explanation invokes multiple small-scale shocks in the inner layers of their radiation-driven stellar winds (e.g. Feldmeier et al. 1997). In recent years has gained importance a plasma heating model known as magnetically channeled wind shock (MCWS) (Schulz et al. 2003; Owocki et al. 2005;

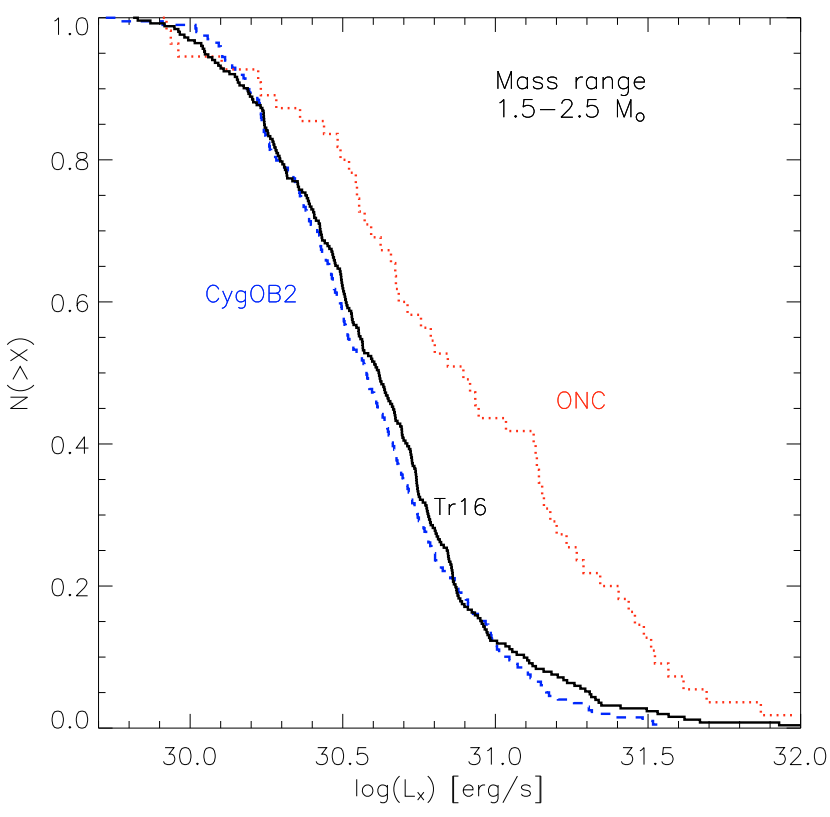

Fig. 10. Cumulative distributions of $X$-ray luminosities for Trumpler 16 low-mass stars with masses $1.5 \leq M / M_{\odot} \leq 2.5$ (thick solid line). Dotted and dashed lines represent the $L_{\mathrm{x}}$ cumulative distributions for ONC and CygOB2, respectively, in the same mass range.

Gagné et al. 2005b). Moreover, WR+OB and/or O+OB interacting wind binary systems produce an excess of $\mathrm{X}$-ray emission from the CWR (Stevens et al. 1992; Zhekov \& Skinner 2000; Pittard \& Stevens 2002).

Given the relatively large number of massive OB-type stars in the region, it is most relevant to show in Table 4 most appropiate stellar parameters of the massive stars $\left(L_{\mathrm{bol}}, \dot{M}\right.$, and $L_{\text {wind }}$ computed as $\left.\frac{1}{2} \dot{M} v_{\infty}^{2}\right)^{28}$. We computed $L_{\mathrm{x}}$ by means of an absorbed (WABS) thermal plasma model (APEC). Metal abundance was fixed at $Z=0.3 Z_{\odot}$ in fitting faint sources ( $<100$ ph.), while it was left as a free parameter for the remaining cases (see Table 4). In Fig. 11 we show the $L_{\mathrm{x}} / L_{\mathrm{bol}}$ relation. In spite of the observed scatter, one sees that the median $L_{\mathrm{x}} / L_{\mathrm{bol}}$ for binaries is about 7 times larger $\left(\sim 8.3 \times 10^{-7}\right)$ than that of suspected single stars $\left(\sim 1.1 \times 10^{-7}\right)^{29}$. Four of the 13 known binaries in the analysis are well-separated systems: the LBV $\eta$ Carinae, a probable long period binary with $P \sim 2026 \pm 2$ days (Daminelli et al. 2007), the Wolf-Rayet star WR25 (HD 93162), a 208-days period binary system (Gamen et al. 2006), and HDE 303308, resolved as a binary system with a component separation of about $\sim 38$ AU projected along the fine guidance sensor (FGS) $y$-axis ${ }^{30}$. The stars $\operatorname{Tr} 16 \# 9(\mathrm{O} 9.5 \mathrm{~V}+$ ?) and $\operatorname{Tr} 16 \# 23(\mathrm{O} 7 \mathrm{~V}+$ ?) show photometric variability probably related to a secondary component (Nelan et al. 2004), we also consider them to be binaries.

Besides the several works (e.g. Seward et al. 1979; Seward \& Chlebowski 1982; Corcoran et al. 1995; Albacete Colombo et al. 2003; Evans et al. 2003, 2004; Sanchawala et al. 2007) about the origin of the observed X-ray emission on massive stars of this region, this goal is far beyond the scope of our paper and so extensively discussed here.

\footnotetext{
${ }^{28}$ Adopted values were taken from Smith (2006, Table 1).

29 Because of observed flare-like variability in Tr16-\#5 and \#11, we suspect that most of X-rays emission comes from a low-mass companion, and therefore we have discarded these sources in computing the median $L_{\mathrm{x}}$.

${ }^{30}$ It was unresolved along the FGS $x$-axis down to 20 AU, suggesting an eccentric orbit.
} 


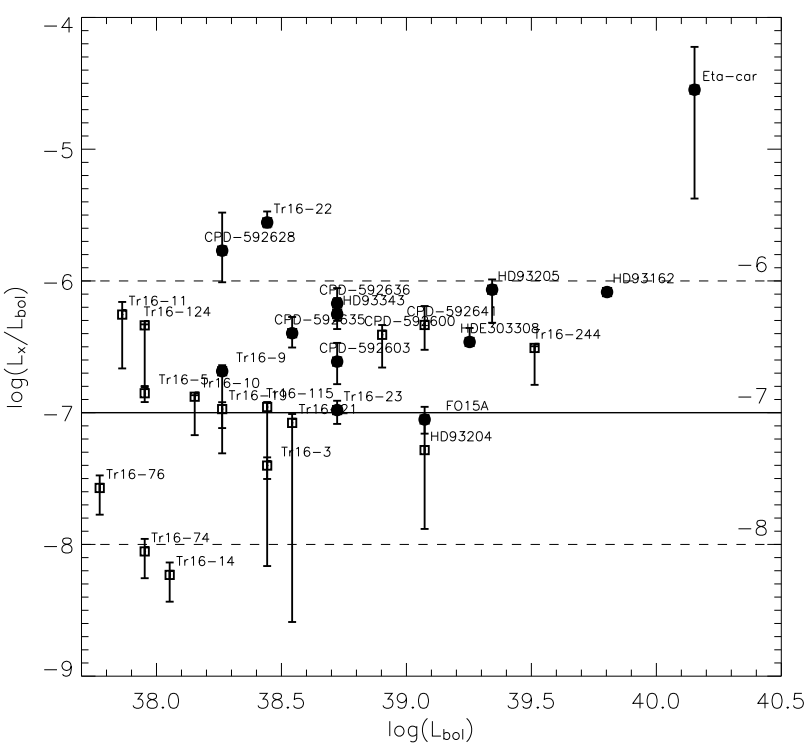

Fig. 11. $\log \left(L_{\mathrm{x}} / L_{\mathrm{bol}}\right)$ versus $\log \left(L_{\mathrm{bol}}\right)$ relation for massive stars in $\operatorname{Tr} 16$. Open boxes refer to suspected single stars and filled circles to known binaries. Notes: $i-$ because of flare-like variability (see Sect. 5.1) observed in $\operatorname{Tr} 16-\# 5$ (B1.5V) and $\operatorname{Tr} 16-\# 11$ (B1V), their X-ray emission is probably dominated by a unknown lowmass star companion. $i i-$ The star $\operatorname{Tr} 16-\# 22(\mathrm{O} 8.5 \mathrm{~V}+?)$ has the highest $\log \left(L_{\mathrm{x}} / L_{\mathrm{bol}}\right)$ ratio $(\sim-5.56)$ among all MS OB-type stars of the region.

\section{Summary and conclusion}

We report here results of a deep Chandra X-ray observation pointed toward the $\sim 3 \mathrm{Myr}$ old star-forming region Trumpler 16 . Source detection was performed using the PWDdetect code, identifying $1035 \mathrm{X}$-ray sources in the $17^{\prime} \times 17^{\prime}$ ACIS-I FOV. Most of these seem to be outside the obscured V-shaped region of dust and gas. Star formation in this part of the masked region has probably been disrupted and/or diminished as the stellar winds are blocked inside the cloud, due to the efficiency of the photo-evaporation processes caused by interactions of the nearby hot massive stars with the dense dust and gas structures.

Data extraction was performed using the semi-automated IDL-based ACIS EXTRACT package, which is well-suited to the analysis of observations of crowded fields such as ours.

The X-ray source list was cross-identified with optical and near-IR (2MASS) catalogs: 28 X-ray sources (of 44 within the FOV) were identified with optically characterized OB members of Trumpler 16 and 760 with 2MASS sources. Among these sources, almost all are believed to be Trumpler 16 members. About 90 X-ray sources without optical/NIR counterparts are estimated to have of extragalactic nature (AGNs), while the remaining X-ray sources with no counterpart are likely to be associated with members that are fainter than the 2MASS completeness limit.

To characterize the previously unidentified likely cluster members with NIR counterparts, we placed them on NIR color-magnitude $\left(K_{\mathrm{S}}\right.$ vs. $\left.H-K_{\mathrm{s}}\right)$ and color-color $\left(H-K_{\mathrm{s}}\right.$ vs. $J-H$ ) diagrams. A first estimate of interstellar extinction was obtained by adopting a $3 \mathrm{Myr}$ isochrone for the low- and intermediate-mass stars and assuming that $\mathrm{O}$ - and early B-type stars lie on the MS. We find a median visual absorption for OB stars of $A_{\mathrm{v}} \sim 2.0 \mathrm{mag}$, while low mass likely members seem to be slightly more absorbed, $A_{\mathrm{v}} \sim 3.6 \mathrm{mag}$. We also use the $3 \mathrm{Myr}$ isochrone and the $J$ magnitude to estimate masses of likely members assuming that they share the same distance and absorption. Our sample of X-ray selected members with near-IR counterparts reaches down to $M=0.5-0.6 M_{\odot}$, and is very likely complete down to $\sim 1.5 M_{\odot}$. From the $H-K_{\mathrm{s}}$ vs. $J-H$ diagram we estimate that $\sim 15 \%(51 / 339)$ of low-mass stars have NIR excesses, finding it to be quite a high percentage with respect to the $2 \mathrm{Myr}$ old Cyg OB2. We believe that the disk fraction in young SFRs is more dependent on the spatial morphology of gas and dust around massive stars, which may enhance photoevaporation, and thus shorten disk lifetimes, than on the total number of massive stars in the region.

At least 77 sources, i.e. $\sim 7.4 \%$, were found to be variable within our observation with a confidence level greater than $99.9 \%$. Only three of the 28 detected O- and early B-type stars were detected as variable during our 90-ks observation, in spite of the high statistics of the OB stars' light curves. These exceptions are the known binary O3.5V+O8V star HD 93205 (our source \#242), showing a rather linear decay of the count rate during the observation plus a short-term variability, the B1.5 V star Tr16-11 (source \#136), and the B1V star Tr16-5 (source \#489). The last two show a flare-like variability probably related to unresolved low-mass companions.

We modeled the ACIS X-ray spectra of sources with more than 20 photons and $f_{\text {cont }}<1$. We assumed an absorbed singlecomponent thermal emission model. The median $\log \left(N_{\mathrm{H}}\right)$ of the sources is $21.73\left(\mathrm{~cm}^{-2}\right)$. This value agrees well with the median $A_{\mathrm{v}}$ computed from the near-IR diagram. The median $k T$ of low-mass stars is $2.6 \mathrm{keV}$. Sources associated with O- and early B-type stars are instead quite soft (median $k T: 0.60 \mathrm{keV}$ ). Absorption-corrected X-ray luminosities of OB stars were calculated from the best-fit spectral models. O and B-type stars are the most luminous, with $L_{\mathrm{x}}=2.5 \times 10^{30}-6.3 \times 10^{33} \mathrm{erg} \mathrm{s}^{-1}$. Their X-ray and bolometric luminosities roughly agree with the relation $L_{\mathrm{x}} / L_{\mathrm{bol}}=10^{-7}$, albeit with an order of magnitude dispersion. Low-mass stars have $L_{\mathrm{x}}$ ranging between $10^{30}$ and $10^{31} \mathrm{erg} \mathrm{s}^{-1}\left(\right.$ median $\left.L_{\mathrm{x}}=2.8 \times 10^{30}\right)$. Variable low mass stars are on average 0.5 dex brighter $\left(\log \left(L_{\mathrm{x}}\right) \sim 31.0 \mathrm{erg} \mathrm{s}^{-1}\right)$. These $\mathrm{X}$-ray luminosities are consistent with those of similar mass stars in the slightly younger (2 Myr) Cyg OB2 region. However, in the mass range $1.5-2.5 M_{\odot}$, the ONC (1 Myr) shows a higher X-ray activity level than observed in Trumpler 16 stars in the same mass range. We believe that the age- $L_{\mathrm{x}}$ activity connection is an acceptable explanation of this result.

Acknowledgements. We thank the referee, Marc Gagne, for his time and many useful comments that improved this work. This publication makes use of data products from the Two Micron All Sky Survey, which is a joint project of the University of Massachusetts and the Infrared Processing and Analysis Center/California Institute of Technology, funded by the National Aeronautics and Space Administration and the National Science Foundation. J.F.A.C. acknowledges support by the Marie Curie Fellowship Contract No. MTKD-CT2004-002769 of the project "The Influence of Stellar High Energy Radiation on Planetary Atmospheres", and the host institution INAF - Osservatorio Astronomico di Palermo (OAPA). J.F.A.C. is a researcher member of the Consejo Nacional de Investigaciones Científicas y Tecnológicas (CONICET)Argentina and acknowledges support from this institution. G.M., F.D. and S.S. acknowledge financial support from the Ministero dell'Università e della Ricerca research grants, and ASI/INAF Contract I/023/05/0.

\section{References}

Albacete Colombo, J. F., Méndez, M., \& Morrell, N. I. 2003, MNRAS, 346, 704 Albacete Colombo, J. F., Flaccomio, E., Micela, G., Sciortino, S., \& Damiani, F. 2007, A\&A, 464, 211

Alexander, D. M., Bauer, F. E., Brandt, W. N., et al. 2003, AJ, 126, 539

Anders, E., \& Grevesse, N. 1989, Geochim. Cosmochim. Acta, 53, 197

Antokhin, I. I., Owocki, S. P., \& Brown, J. C. 2004, ApJ, 611, 434

Arnaud, K. 2004, AAS/High Energy Astrophysics Division, 8

Barger, A. J., Cowie, L. L., Capak, P., et al. 2003, AJ, 126, 632 
Bessell, M. S., \& Brett, J. M. 1989, Infrared Extinction and Standardization, LNP, 341, 61

Brooks, K. J., Whiteoak, J. B., \& Storey, J. W. V. 1998, PASA, 15, 202

Brooks, K. J., Storey, J. W. V., \& Whiteoak, J. B. 2001, MNRAS, 327, 46

Broos, P., Townsley, L., Getman, K., \& Bauer, F. 2002, ACIS Extract, An ACIS Point Source Extraction Package, http://www.astro.psu.edu/ xray/docs/TARA/

Carraro, G., Romaniello, M., Ventura, P., \& Patat, F. 2004, A\&A, 418, 525

Corcoran, M. F., Swank, J., Rawley, G., et al. 1995, in Rev. Mex. Astron. Astrofis. Conf. Ser., ed. V. Niemela, N. Morrell, \& A. Feinstein, 2, 97

Damiani, F., Maggio, A., Micela, G., \& Sciortino, S. 1997a, ApJ, 483, 350

Damiani, F., Maggio, A., Micela, G., \& Sciortino, S. 1997b, ApJ, 483, 370

Davidson, K., \& Humphreys, R. M. 1997, ARA\&A, 35, 1

DeGioia-Eastwood, K., Throop, H., Walker, G., \& Cudworth, K. M. 2001, ApJ, 549,578

Evans, N. R., Seward, F. D., Krauss, M. I., et al. 2003, ApJ, 589, 509

Evans, N. R., Schlegel, E. M., Waldron, W. L., et al. 2004, ApJ, 612, 1065

Favata, F., \& Micela, G. 2003, Space Sci. Rev., 108, 577

Feigelson, E. D., \& Montmerle, T. 1999, ARA\&A, 37, 363

Feigelson, E. D., Broos, P., Gaffney, III, J. A., et al. 2002, ApJ, 574, 258

Feinstein, A. 1995, in Rev. Mex. Astron. Astrofis. Conf. Ser., ed. V. Niemela, N. Morrell, \& A. Feinstein, 2, 57

Feldmeier, A., Puls, J., \& Pauldrach, A. W. A. 1997, A\&A, 322, 878

Flaccomio, E., Micela, G., Sciortino, S., et al. 2005, ApJS, 160, 450

Flaccomio, E., Micela, G., \& Sciortino, S. 2006, A\&A, 455, 903

Gagné, M., Oksala, M. E., Cohen, D. H., et al. 2005a, ApJ, 628, 986

Gagné, M., Oksala, M. E., Cohen, D. H., et al. 2005b, ApJ, 634, 712

Gamen, R., Gosset, E., Morrell, N., et al. 2006, A\&A, 460, 777

Getman, K. V., Flaccomio, E., Broos, P. S., et al. 2005, ApJS, 160, 319

Giacconi, R., Bergeron, J., Borgani, S., et al. 2001, Mem. Soc. Astron. Ital., 72, 831

Grabelsky, D. A., Cohen, R. S., Bronfman, L., \& Thaddeus, P. 1988, ApJ, 331, 181

Güdel, M. 2004, A\&AR, 12, 71

Hägele, G. F., Albacete Colombo, J. F., Barbá, R. H., \& Bosch, G. L. 2004, MNRAS, 355, 1237

Hanson, M. M. 2003, ApJ, 597, 957

Harvin, J. A., Gies, D. R., Bagnuolo, Jr., W. G., Penny, L. R., \& Thaller, M. L. 2002, ApJ, 565, 1216

Hofner, P., \& Churchwell, E. 1997, ApJ, 486, L39

Hofner, P., Delgado, H., Whitney, B., Churchwell, E., \& Linz, H. 2002, ApJ, 579, L95

Hong, J., Schlegel, E. M., \& Grindlay, J. E. 2004, ApJ, 614, 508

Kamata, Y., Koyama, K., Tsuboi, Y., \& Yamauchi, S. 1997, PASJ, 49, 461

Kenyon, S. J., \& Hartmann, L. 1995, ApJS, 101, 117

Knödlseder, J. 2000, A\&A, 360, 539

Luo, D., McCray, R., \& Mac Low, M.-M. 1990, ApJ, 362, 267

Maggio, A., Sciortino, S., Vaiana, G. S., et al. 1987, ApJ, 315, 687
Marino, A., Micela, G., Peres, G., \& Sciortino, S. 2003, A\&A, 407, L63 Massey, P., \& Johnson, J. 1993, AJ, 105, 980

Meyer, M. R., Calvet, N., \& Hillenbrand, L. A. 1997, AJ, 114, 288

Montmerle, T., Grosso, N., Tsuboi, Y., \& Koyama, K. 2000, ApJ, 532, 1097

Morrell, N. I., Barbá, R. H., Niemela, V. S., et al. 2001, MNRAS, 326, 85

Morrison, R., \& McCammon, D. 1983a, ApJ, 270, 119

Morrison, R., \& McCammon, D. 1983b, ApJ, 270, 119

Nelan, E. P., Walborn, N. R., Wallace, D. J., et al. 2004, AJ, 128, 323

Niemela, V. S., Morrell, N. I., Fernández Lajús, E., et al. 2006, MNRAS, 367, 1450

Owocki, S. P., \& Cohen, D. H. 1999, ApJ, 520, 833

Owocki, S., Townsend, R., \& Ud-Doula, A. 2005, in Magnetic Fields in the Universe: From Laboratory and Stars to Primordial Structures, ed. E. M. de Gouveia dal Pino, G. Lugones, \& A. Lazarian, AIP Conf. Proc., 784, 239

Pittard, J. M., \& Stevens, I. R. 2002, A\&A, 388, L20

Predehl, P., \& Schmitt, J. H. M. M. 1995, A\&A, 293, 889

Preibisch, T. 2003, A\&A, 401, 543

Preibisch, T., \& Feigelson, E. D. 2005, ApJS, 160, 390

Preibisch, T., Kim, Y.-C., Favata, F., et al. 2005, ApJS, 160, 401

Press, W. H., Teukolsky, S. A., Vetterling, W. T., \& Flannery, B. P. 1992, Numerical recipes in FORTRAN. The art of scientific computing (Cambridge: University Press), 2nd ed.

Prestwich, A. H., Irwin, J. A., Kilgard, R. E., et al. 2003, ApJ, 595, 719

Sanchawala, K., Chen, W.-P., Lee, H.-T., et al. 2007, ApJ, 656, 462

Schulz, N. S., Hasinger, G., \& Truemper, J. 1989, A\&A, 225, 48

Schulz, N. S., Canizares, C., Huenemoerder, D., \& Tibbets, K. 2003, ApJ, 595, 365

Schulz, N. S., Testa, P., Huenemoerder, D. P., Ishibashi, K., \& Canizares, C. R. 2006, ApJ, 653, 636

Seward, F. D., \& Chlebowski, T. 1982, ApJ, 256, 530

Seward, F. D., Forman, W. R., Giacconi, R., et al. 1979, ApJ, 234, L55

Siess, L., Dufour, E., \& Forestini, M. 2000, A\&A, 358, 593

Smith, N. 2006, MNRAS, 367, 763

Smith, N., Egan, M. P., Carey, S., et al. 2000, ApJ, 532, L145

Smith, N., Bally, J., \& Morse, J. A. 2003, ApJ, 587, L105

Smith, N., Barbá, R. H., \& Walborn, N. R. 2004, MNRAS, 351, 1457

Smith, R. K., Brickhouse, N. S., Liedahl, D. A., \& Raymond, J. C. 2001, ApJ, 556, L91

Stevens, I. R., Blondin, J. M., \& Pollock, A. M. T. 1992, ApJ, 386, 265

Tapia, M., Roth, M., Vázquez, R. A., \& Feinstein, A. 2003, MNRAS, 339, 44

Townsley, L. K. 2006, ArXiv Astrophysics e-prints

Vuong, M. H., Montmerle, T., Grosso, N., et al. 2003, A\&A, 408, 581

Walborn, N. R. 1995, in Rev. Mex. Astron. Astrofis. Conf. Ser., 2, ed. V. Niemela, N. Morrell, \& A. Feinstein, Rev. Mex. Astron. Astrofis., 27, 51

Walter, F. M. 1992, AJ, 104, 758

Weisskopf, M. C., Brinkman, B., Canizares, C., et al. 2002, PASP, 114, 1

Wolk, S. J., Harnden, Jr., F. R., Flaccomio, E., et al. 2005, ApJS, 160, 423

Zhekov, S. A., \& Skinner, S. L. 2000, ApJ, 538, 808 
J. F. Albacete-Colombo et al.: An X-ray survey of low-mass stars in Trumpler 16 with Chandra, Online Material p 1

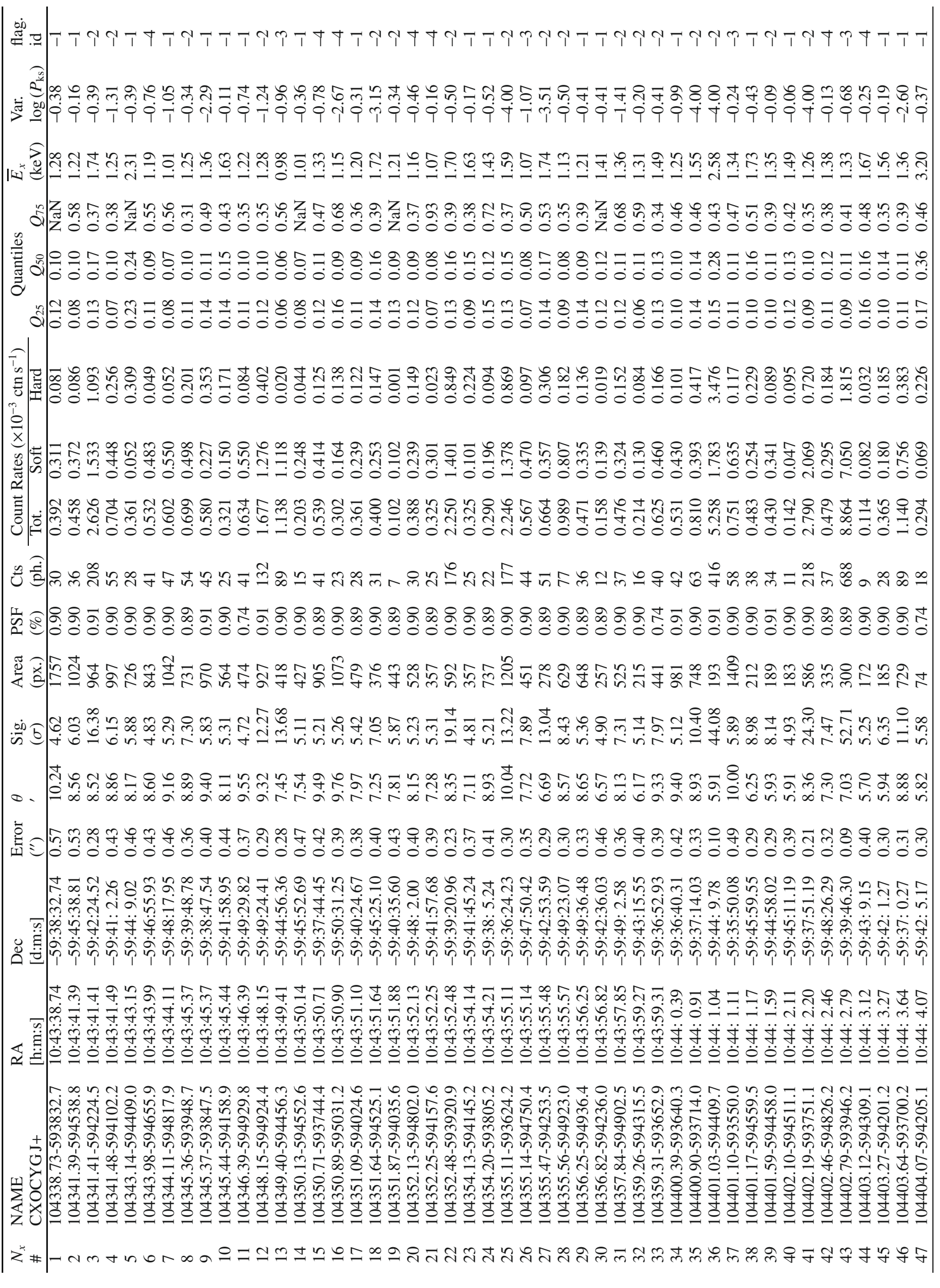


J. F. Albacete-Colombo et al.: An X-ray survey of low-mass stars in Trumpler 16 with Chandra, Online Material p 2

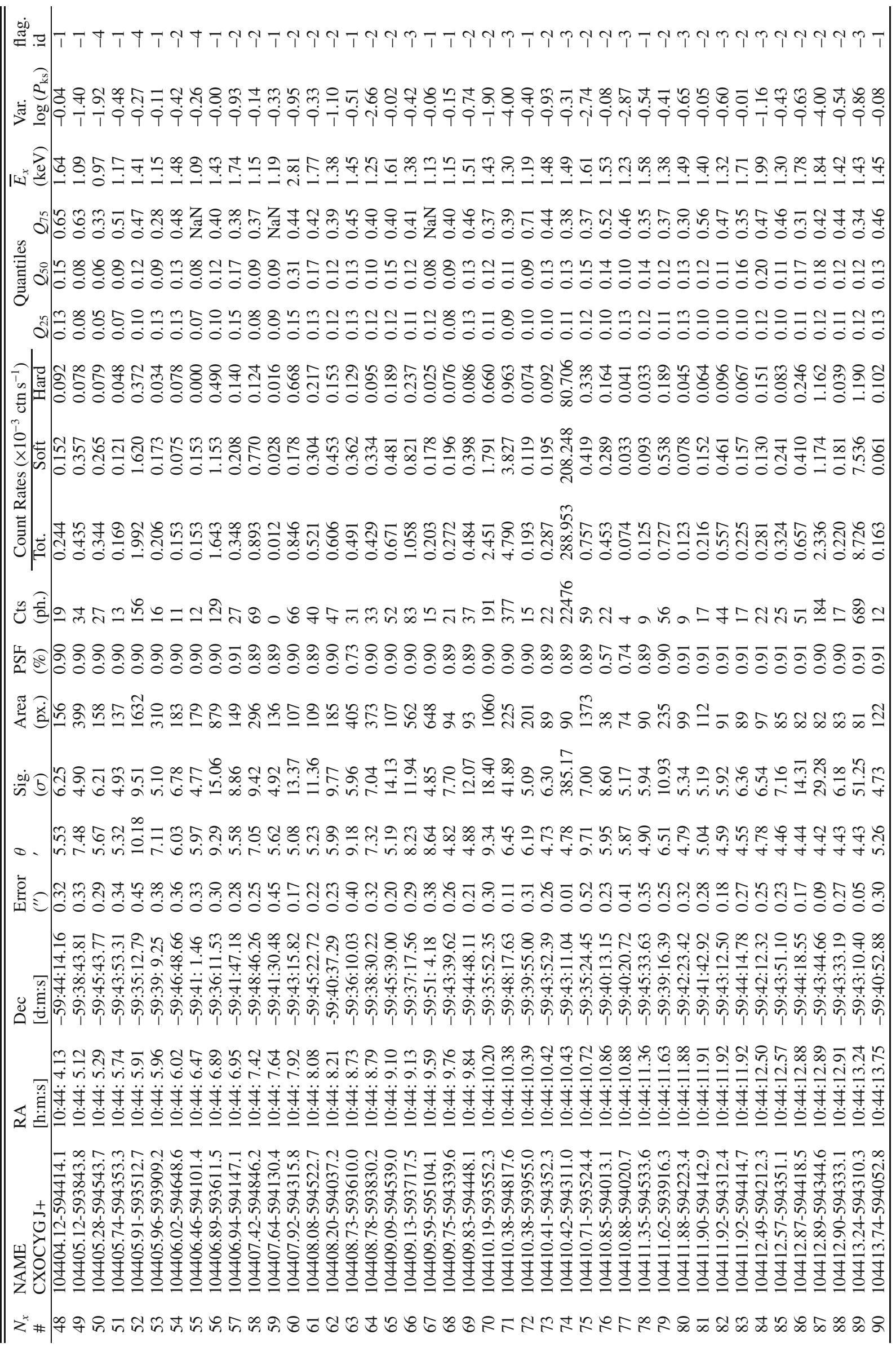


J. F. Albacete-Colombo et al.: An X-ray survey of low-mass stars in Trumpler 16 with Chandra, Online Material p 3

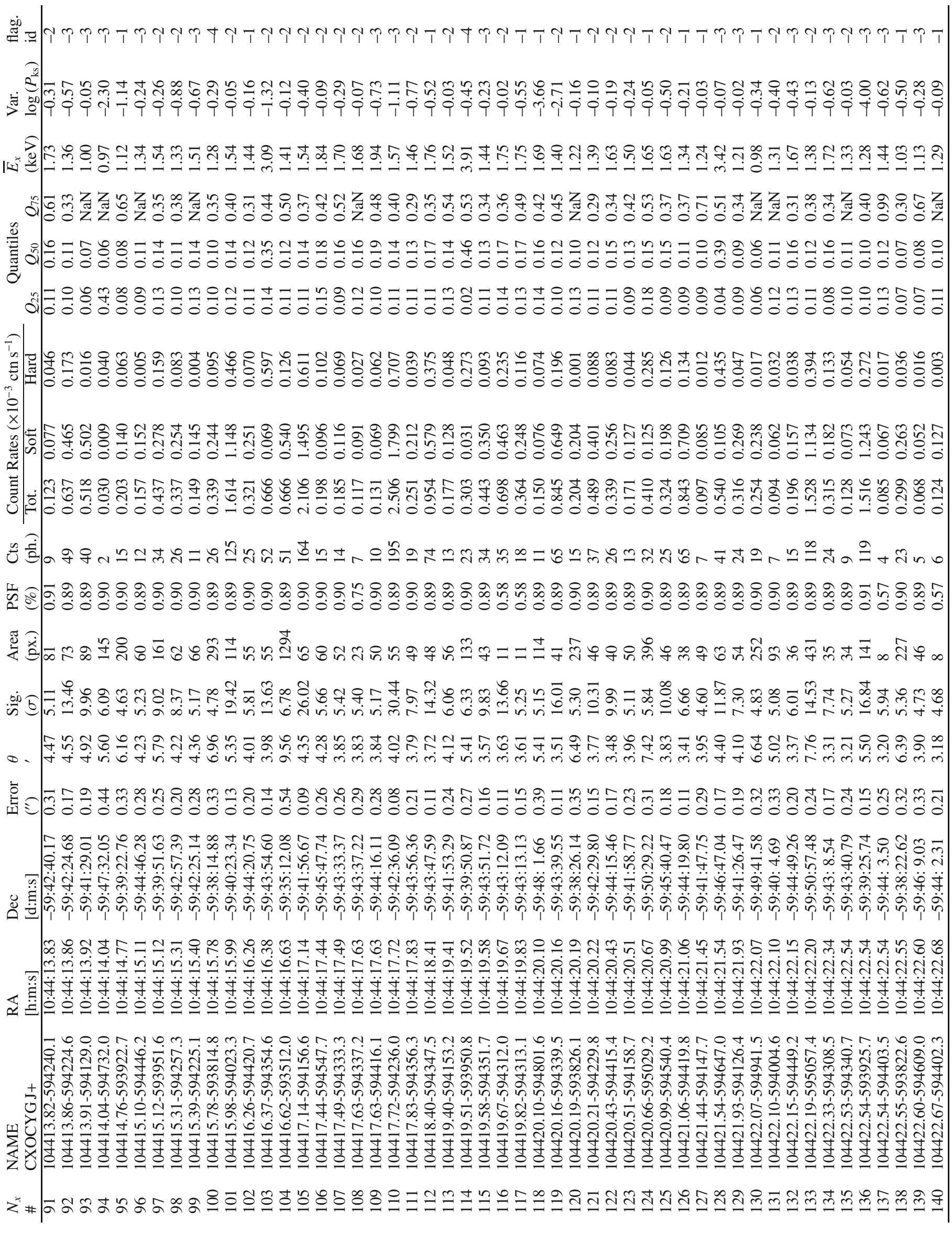


J. F. Albacete-Colombo et al.: An X-ray survey of low-mass stars in Trumpler 16 with Chandra, Online Material p 4

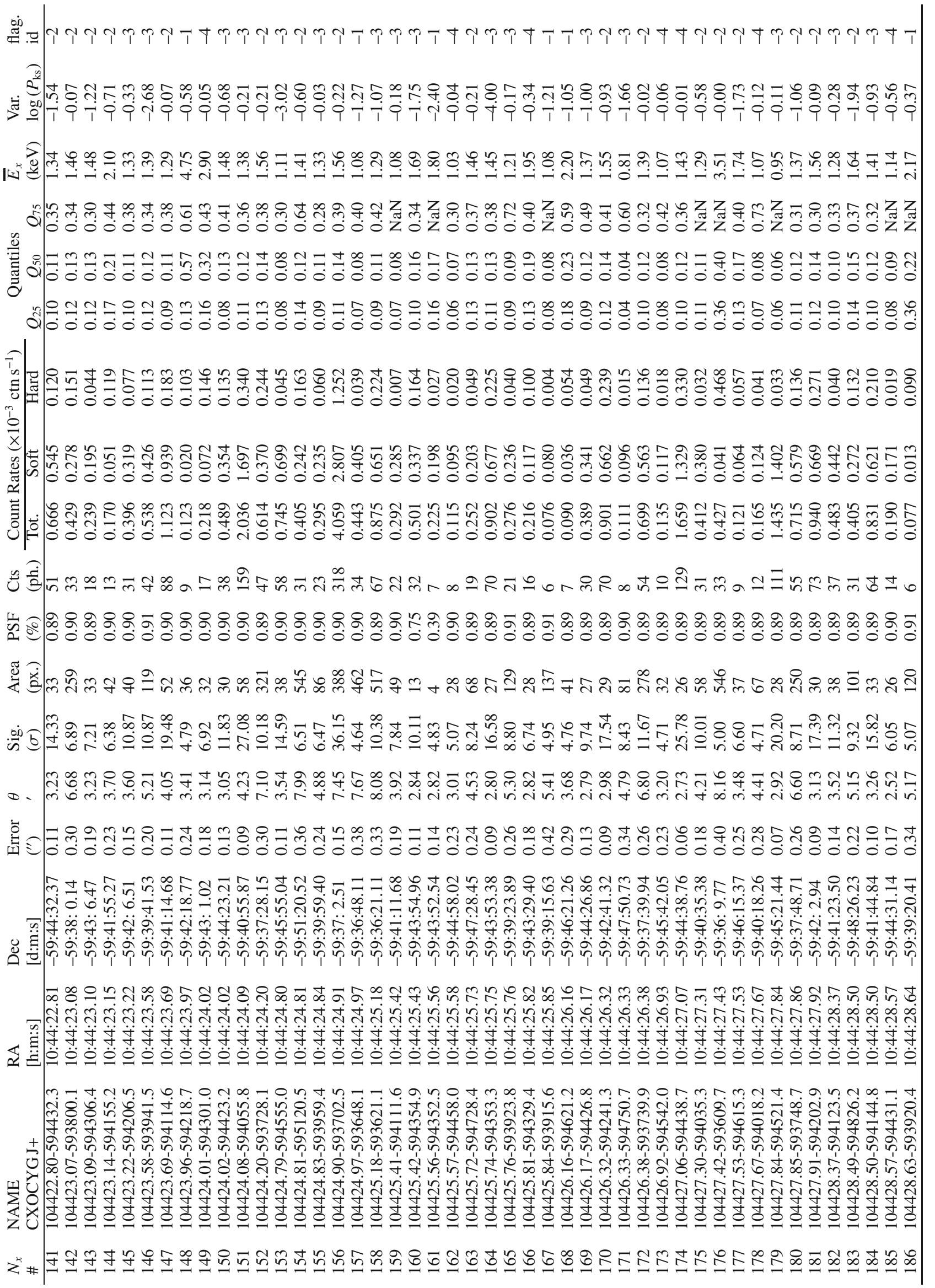


J. F. Albacete-Colombo et al.: An X-ray survey of low-mass stars in Trumpler 16 with Chandra, Online Material p 5

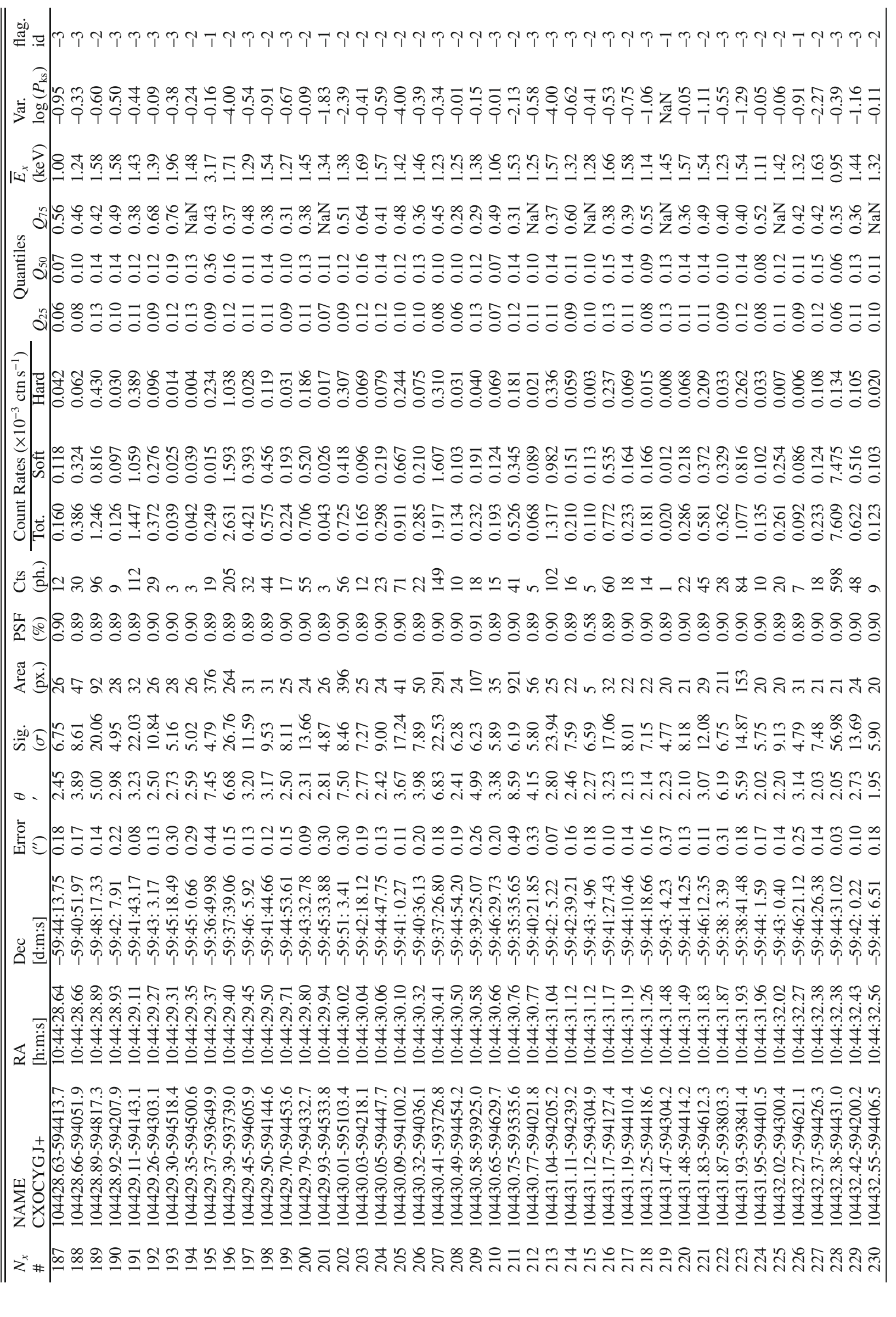


J. F. Albacete-Colombo et al.: An X-ray survey of low-mass stars in Trumpler 16 with Chandra, Online Material p 6

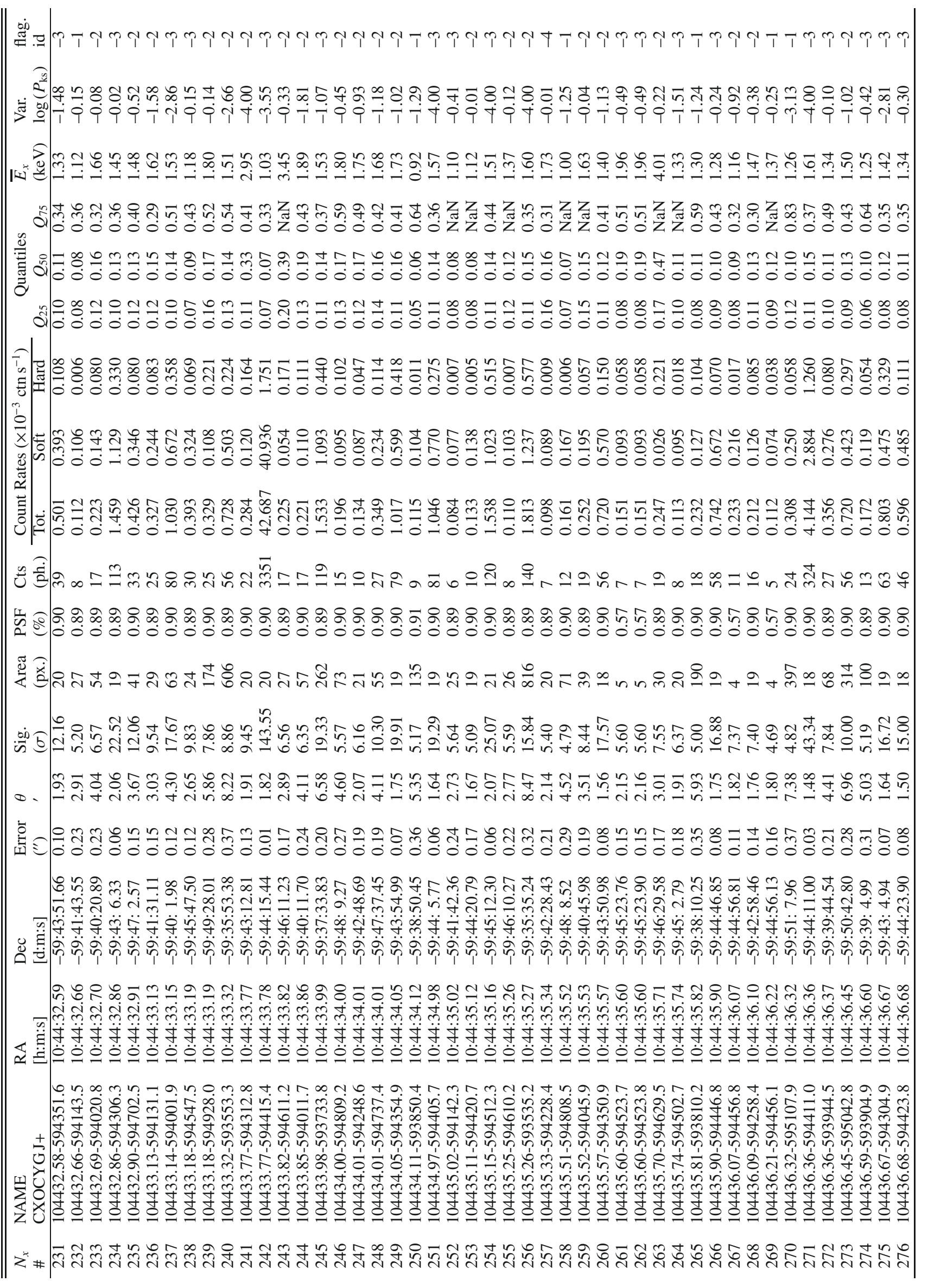


J. F. Albacete-Colombo et al.: An X-ray survey of low-mass stars in Trumpler 16 with Chandra, Online Material p 7

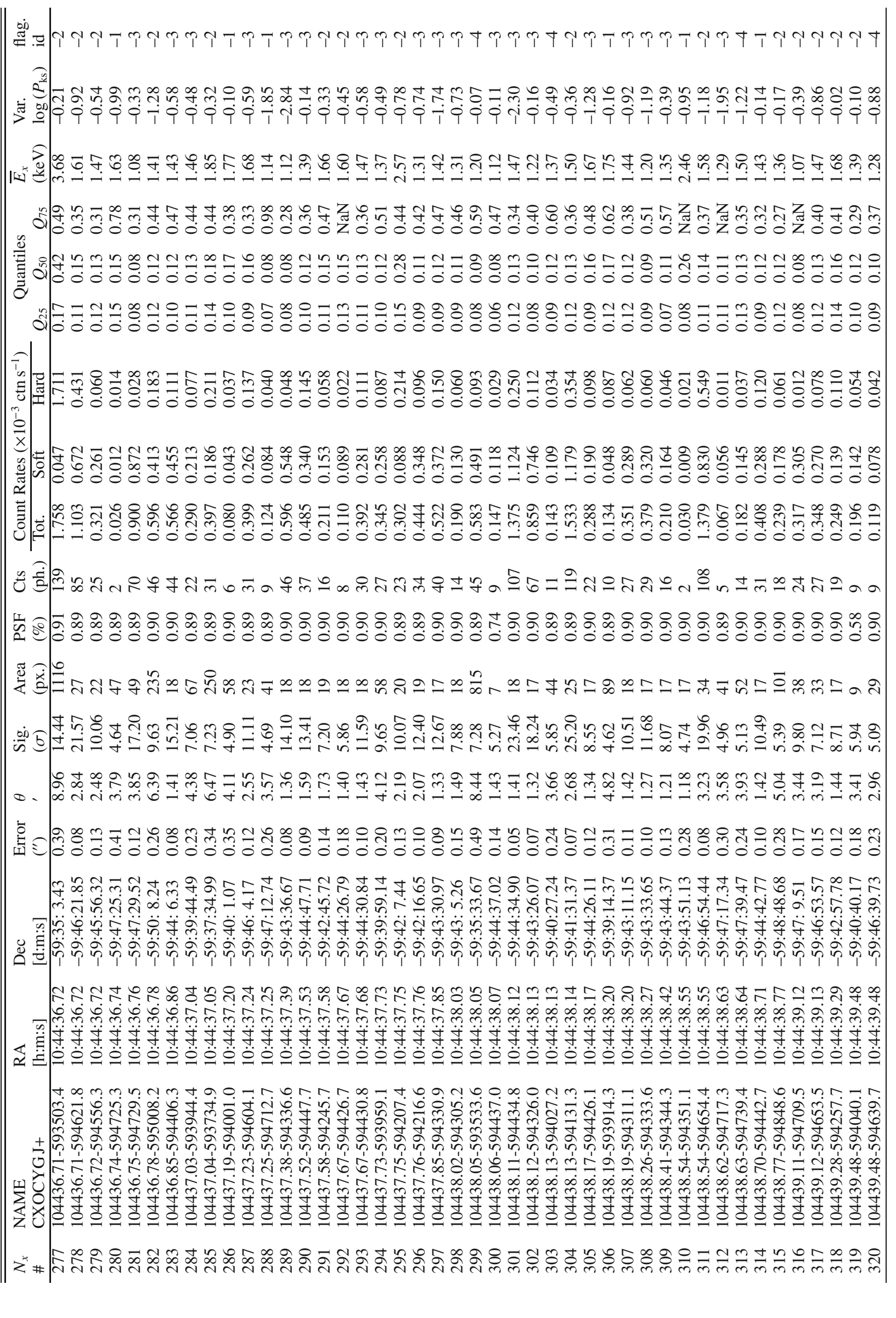


J. F. Albacete-Colombo et al.: An X-ray survey of low-mass stars in Trumpler 16 with Chandra, Online Material p 8

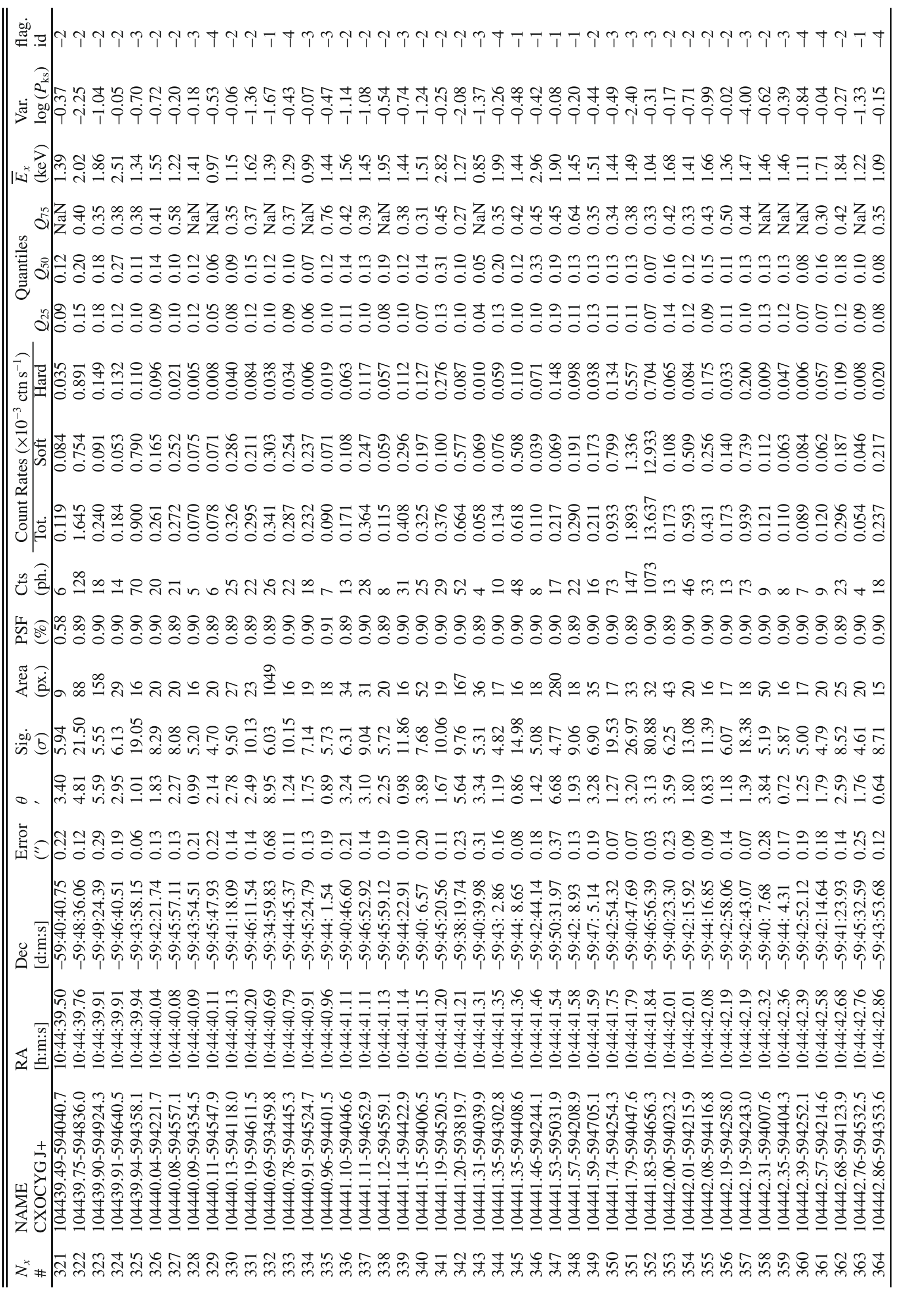


J. F. Albacete-Colombo et al.: An X-ray survey of low-mass stars in Trumpler 16 with Chandra, Online Material p 9

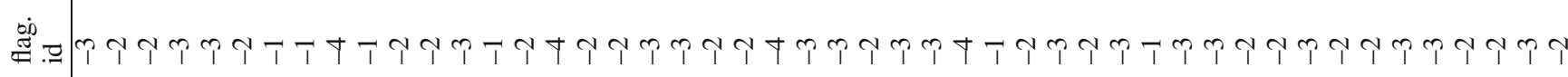

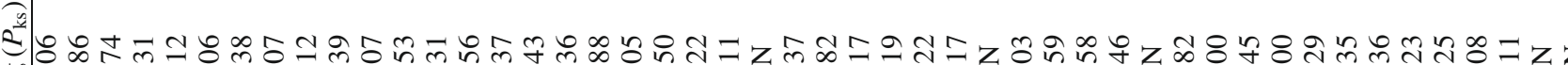

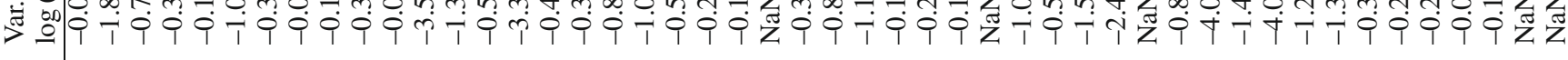

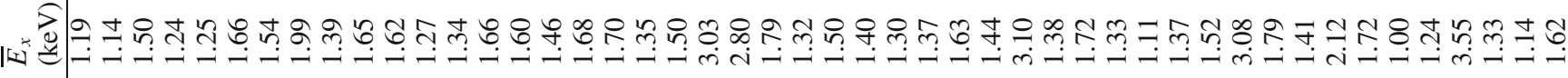

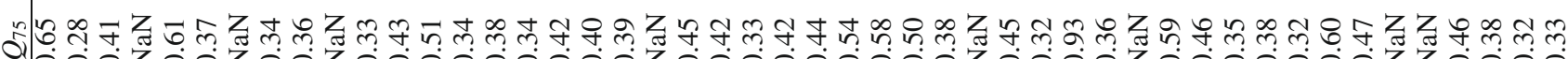

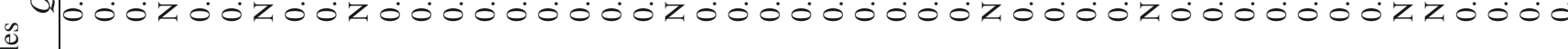

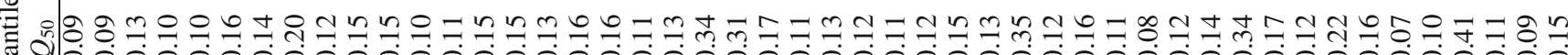
ప 0000000000000000000000000000000000000000000000000

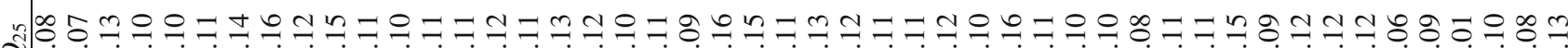
人 000000000000000000000000000000000000000000000000 T

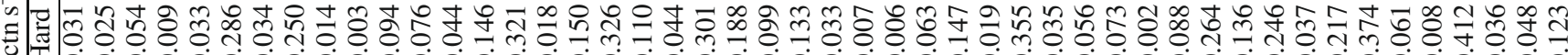
$\frac{\hat{b}}{x}$

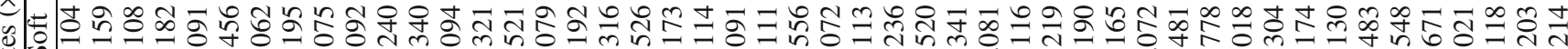
击 司粮 U Uี 步 \& 娄

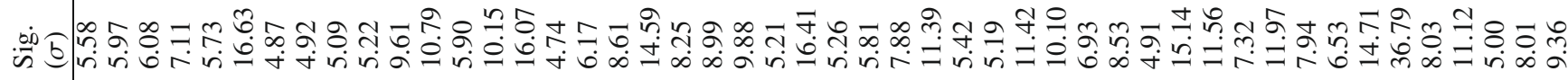

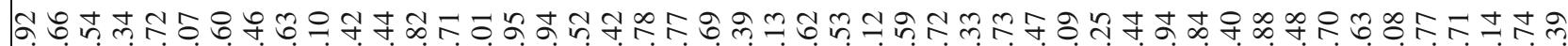

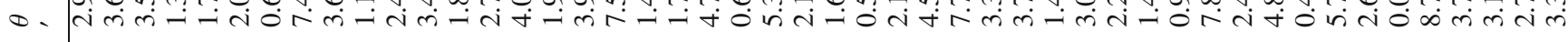

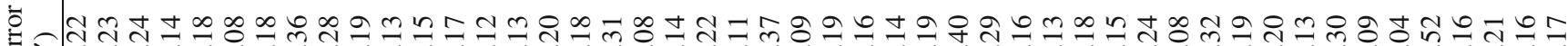

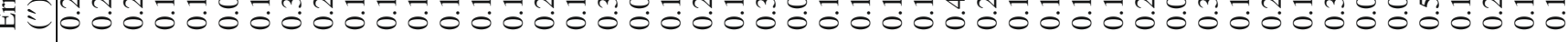

궁

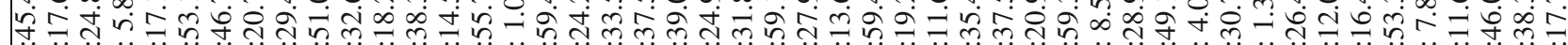

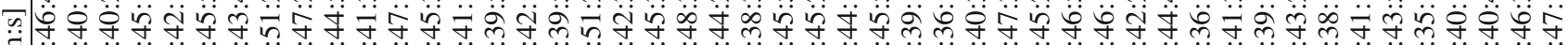

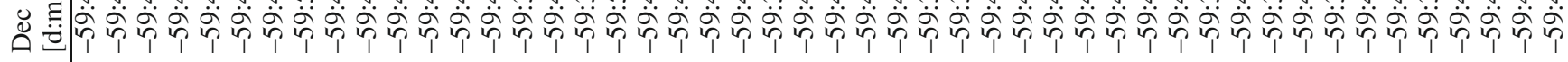

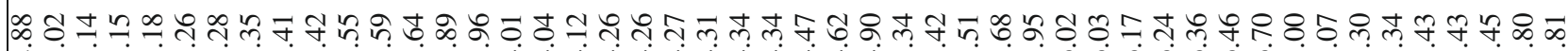

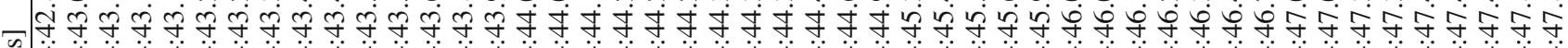

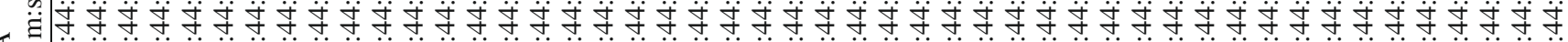

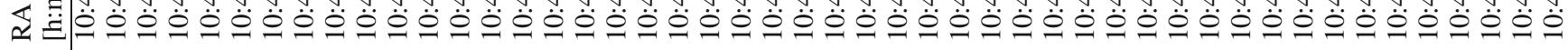

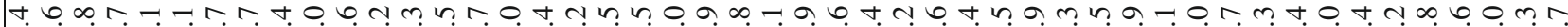

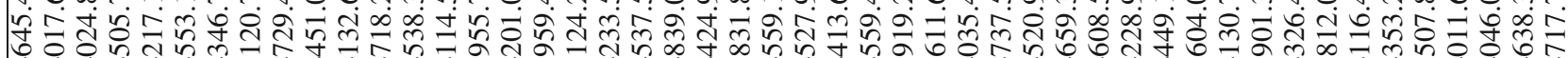

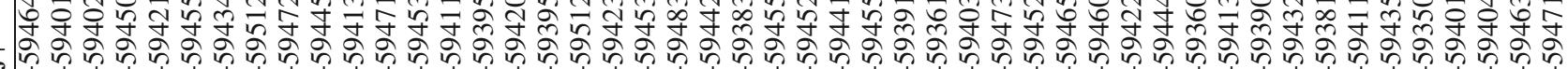

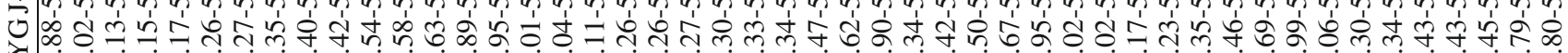

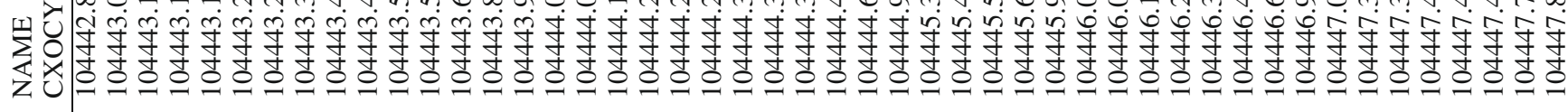

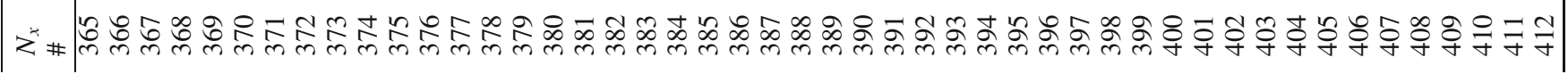



总

公

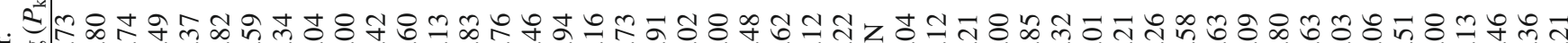
㟧

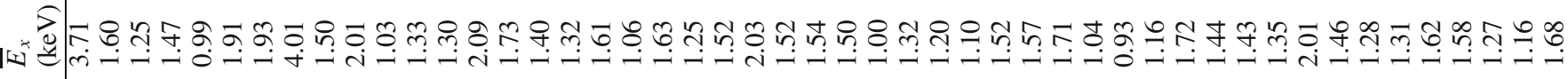

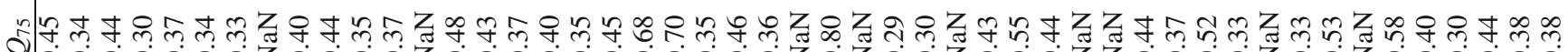
Q

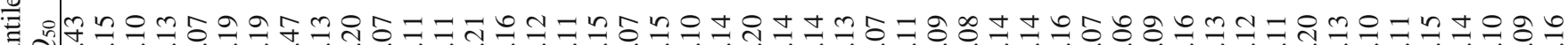
a

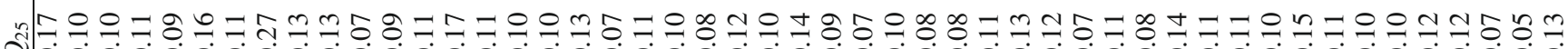

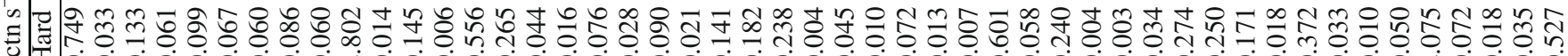
bo

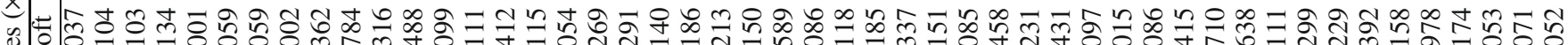

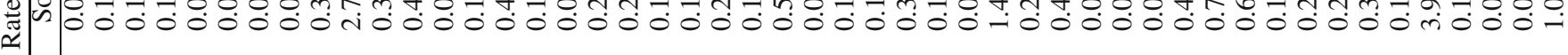

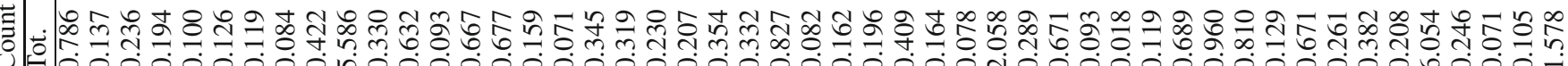
F-0.00000001000000000000000000000400000000000000000-

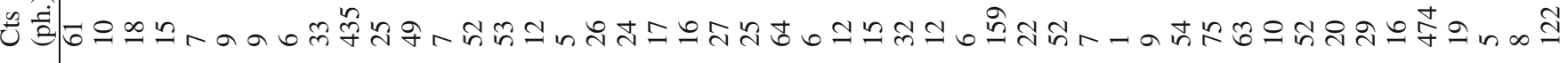

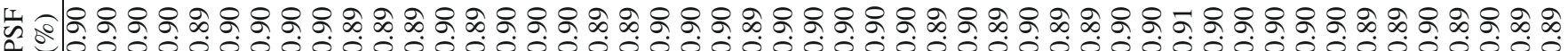

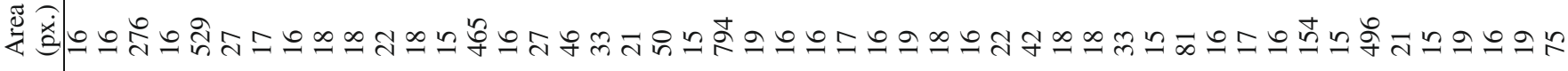

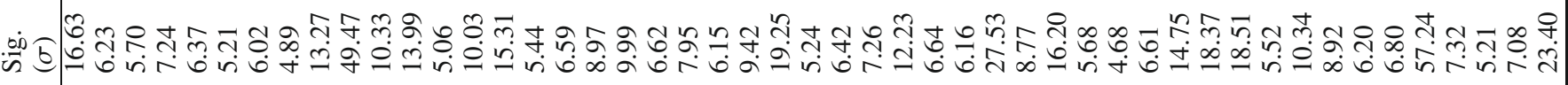

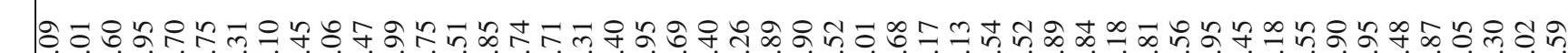

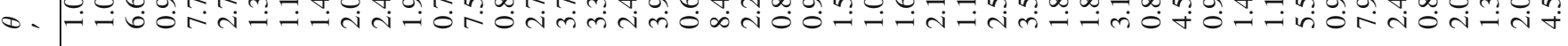

흡 $\overline{0} \div$ ำ

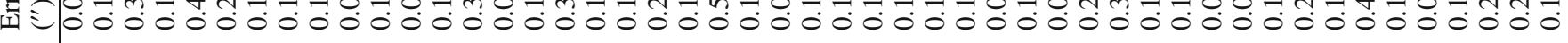

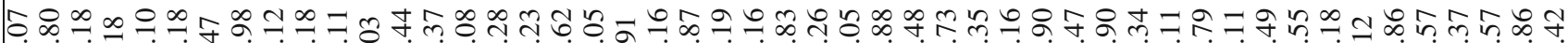

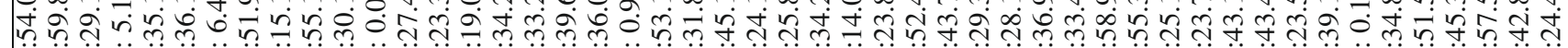

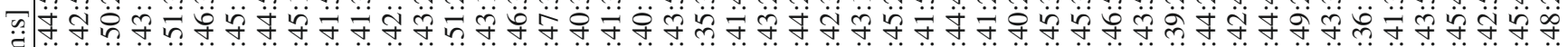

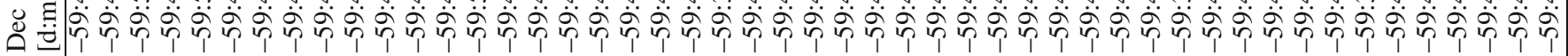

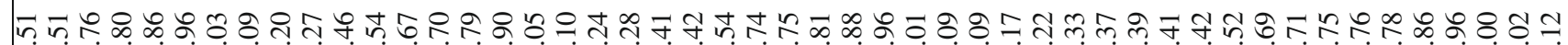
๘

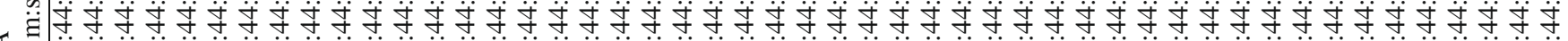

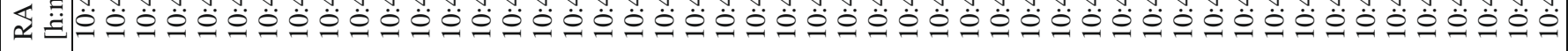

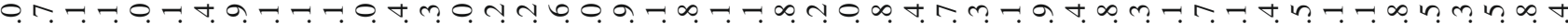
نें

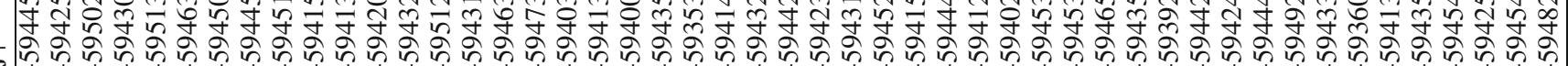
U

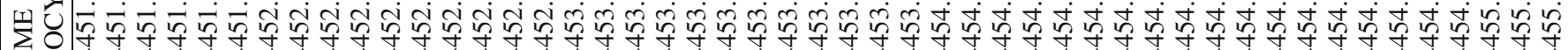

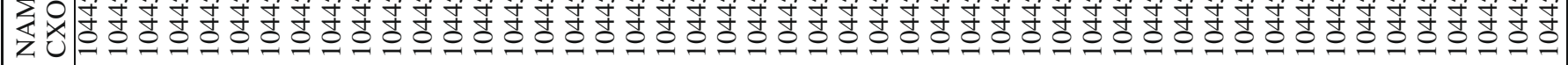

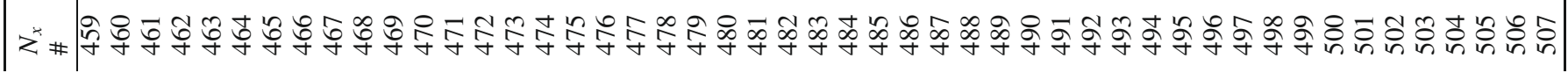




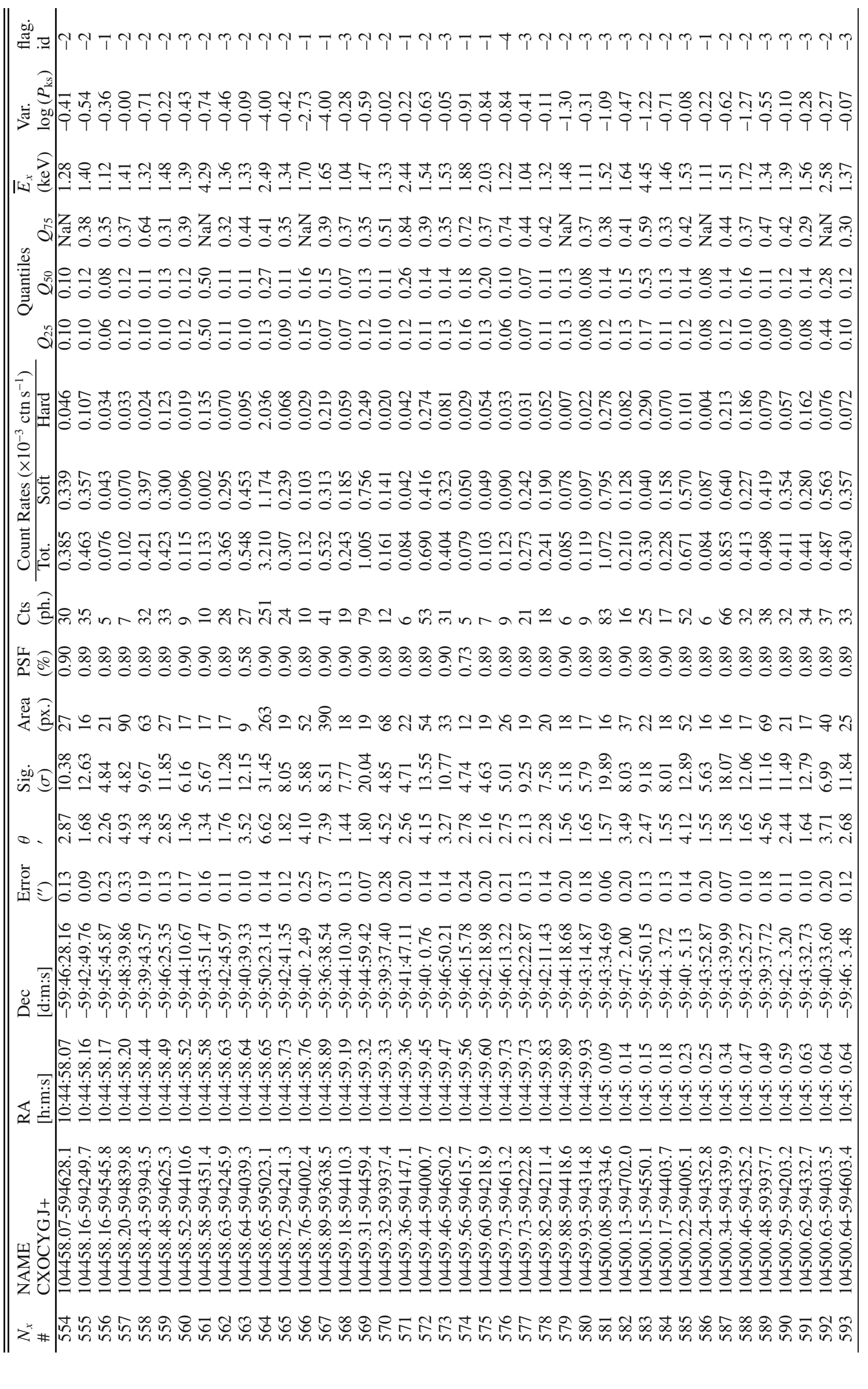




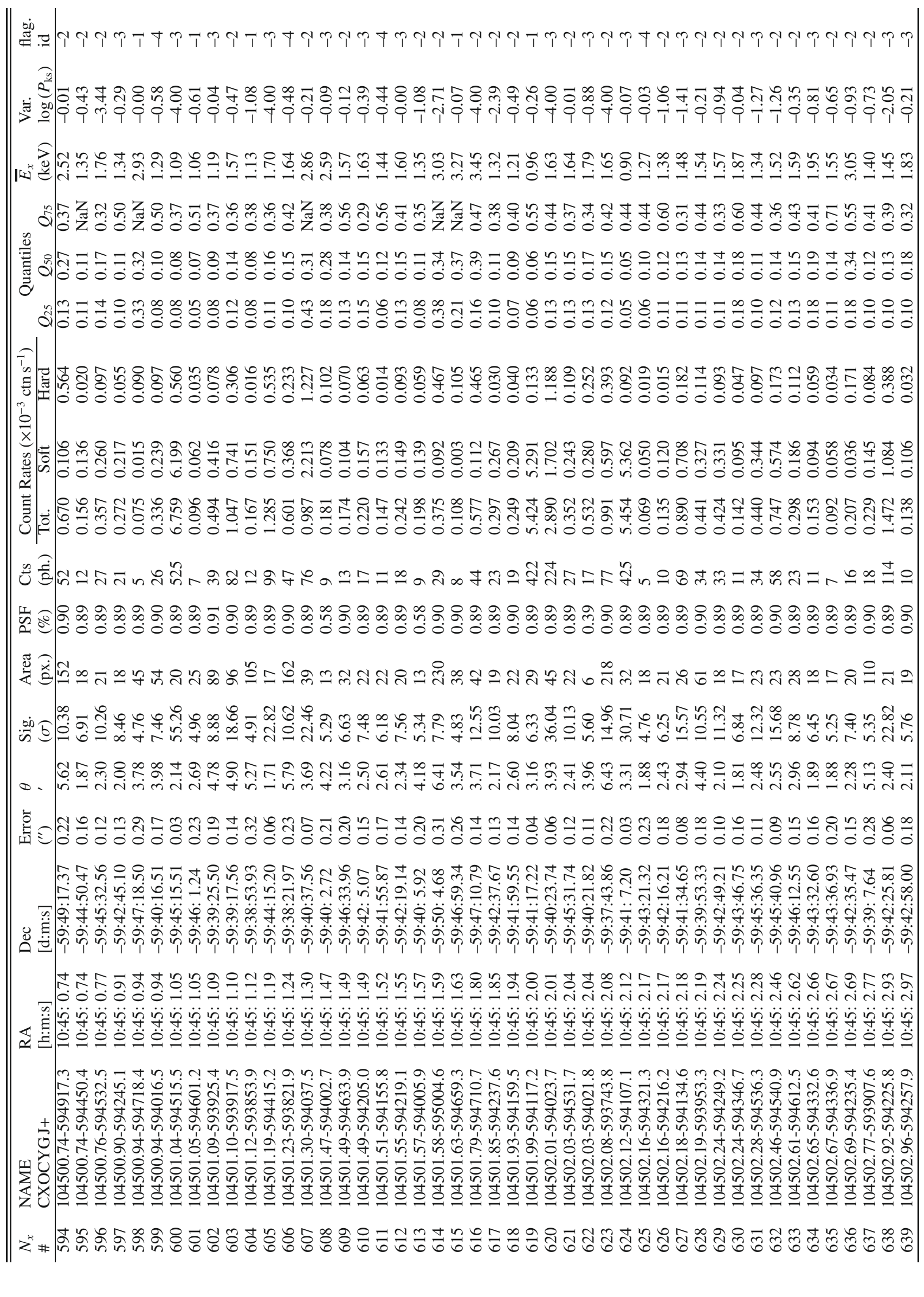




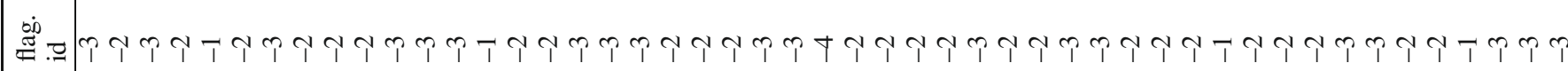

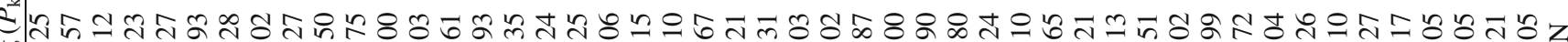
च

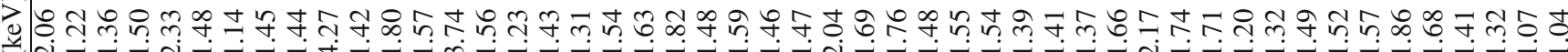

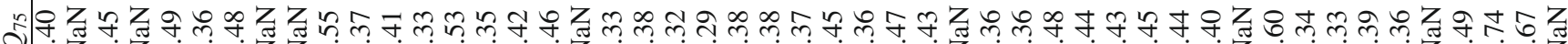

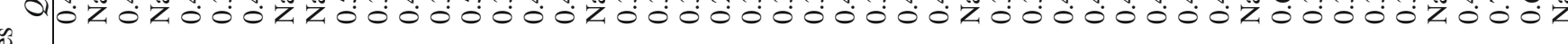
象 焉

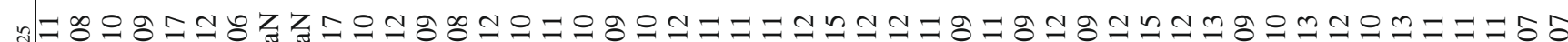

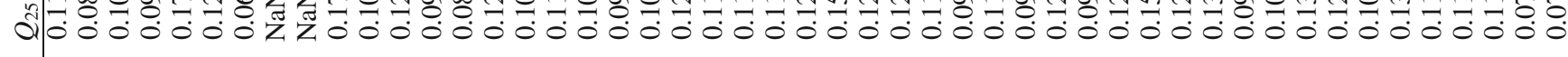

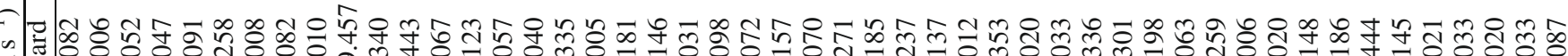

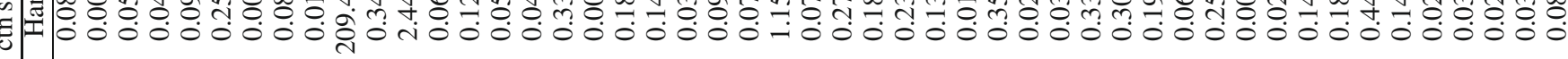

$\frac{0}{2}$

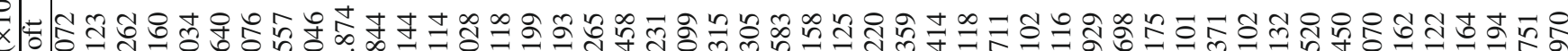

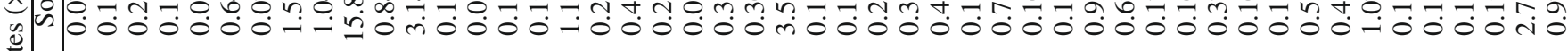

$\simeq$

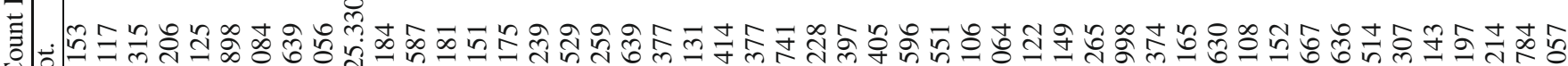
ن

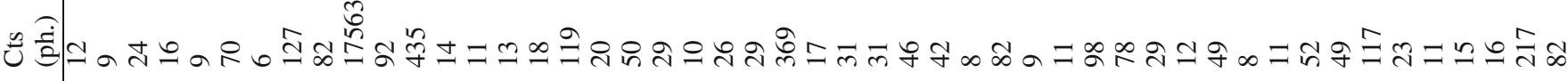

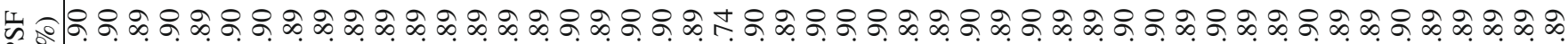

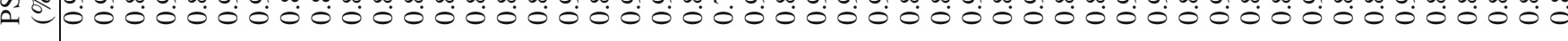

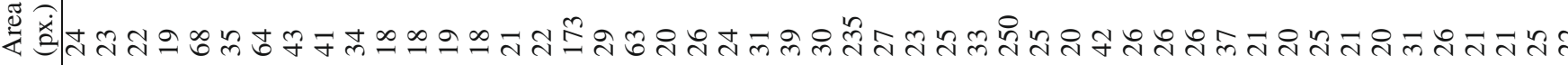

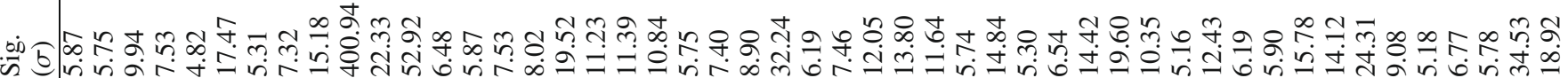

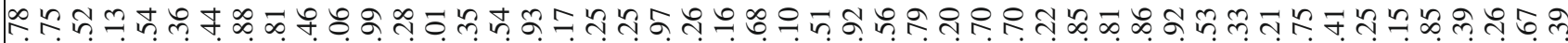

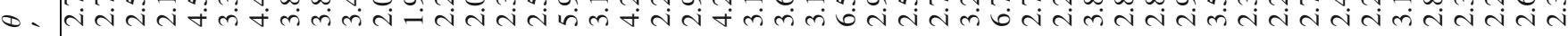

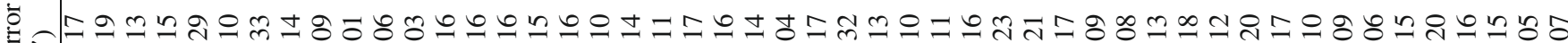
国 $=0000000000000000000000000000000000000000000000000$

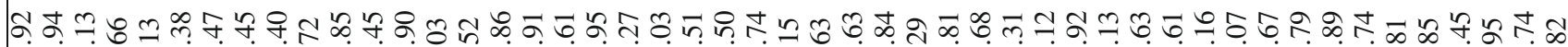
ஸि

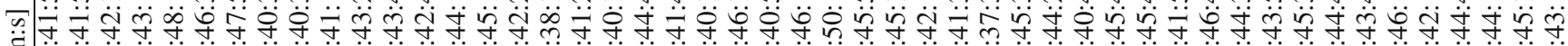

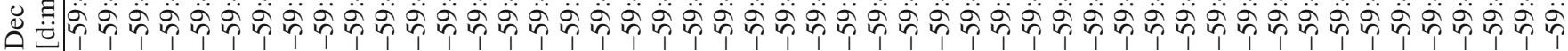

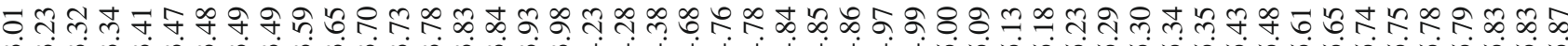

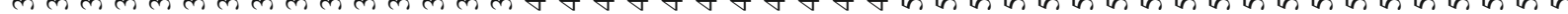

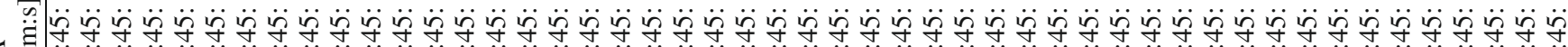

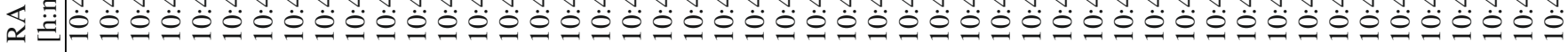

a $\sigma$ -

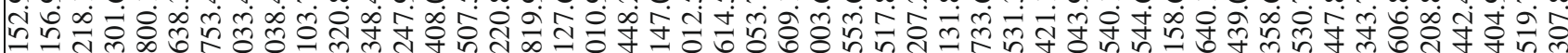

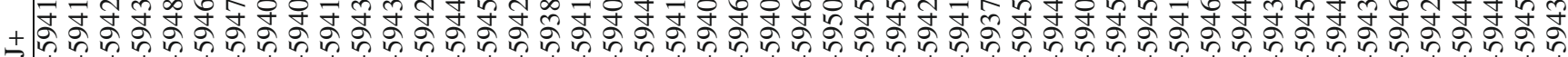

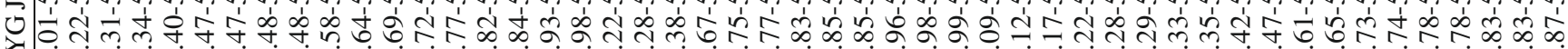

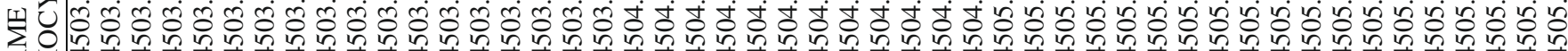

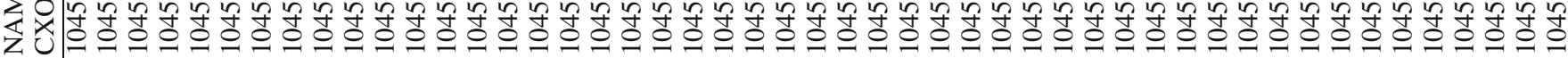

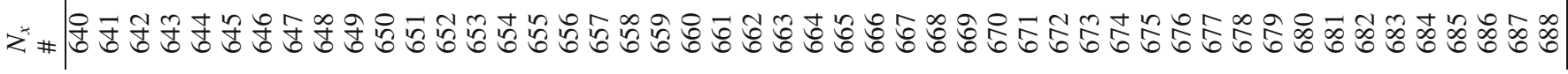


J. F. Albacete-Colombo et al.: An X-ray survey of low-mass stars in Trumpler 16 with Chandra, Online Material p 21

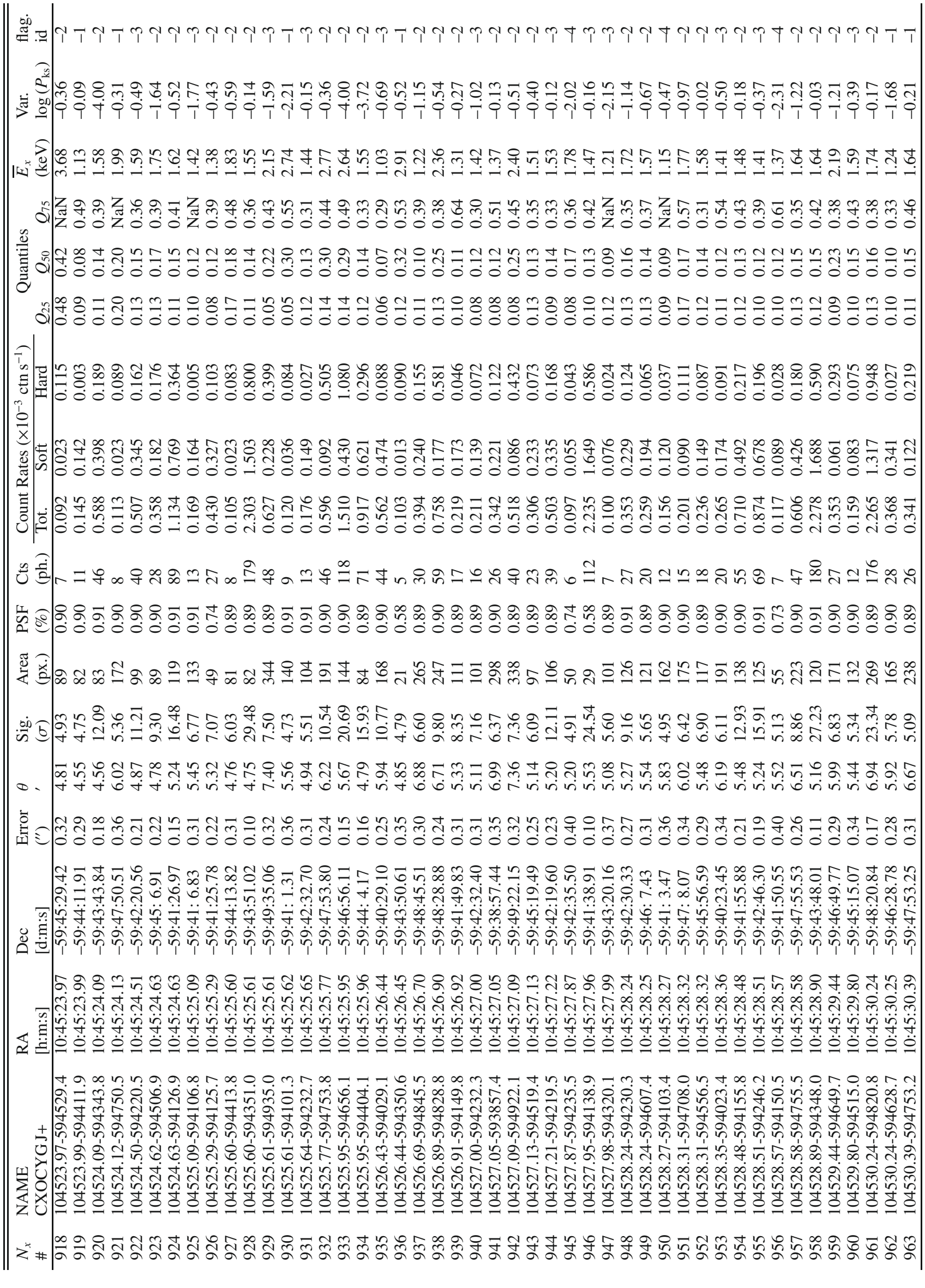




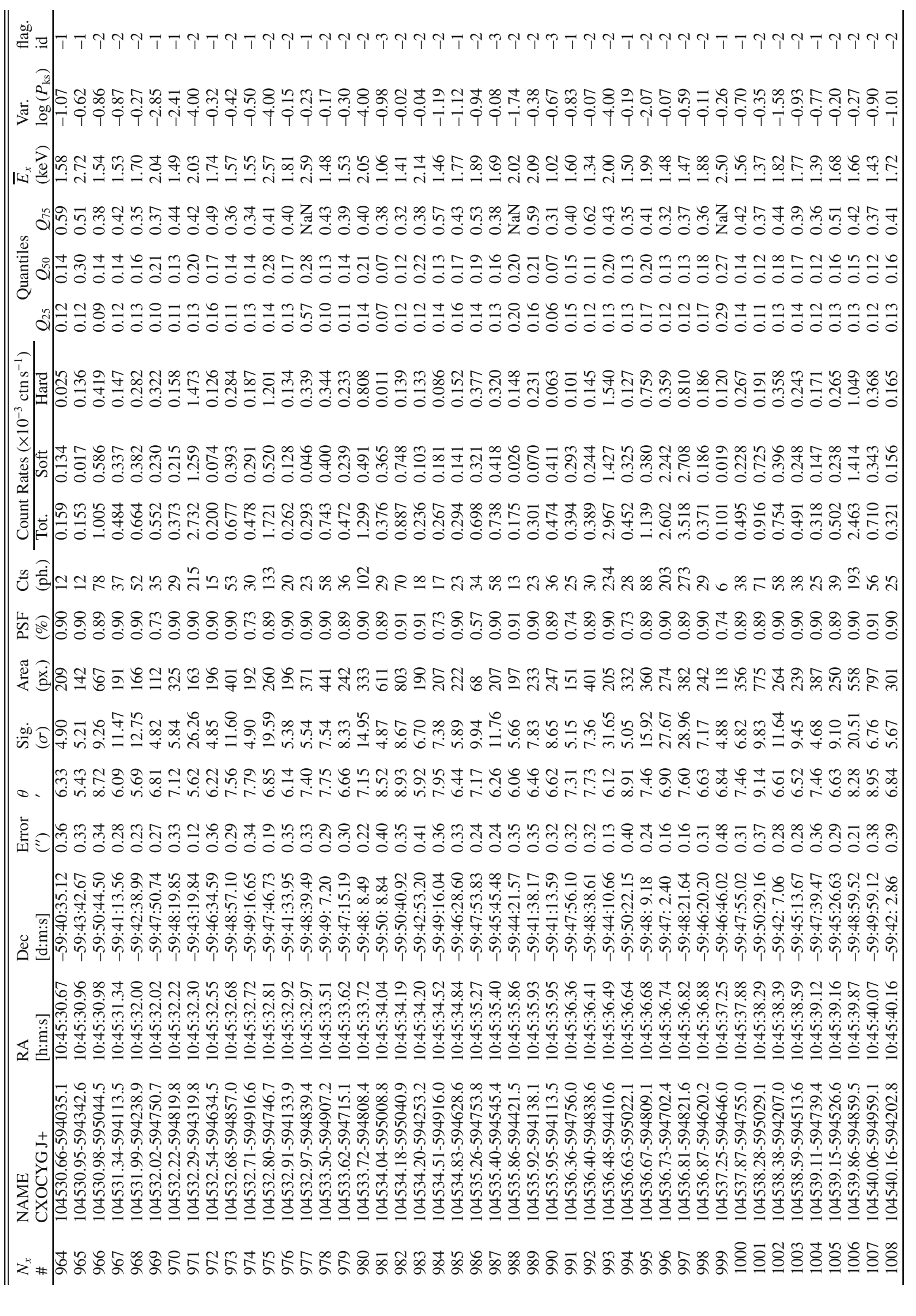


J. F. Albacete-Colombo et al.: An X-ray survey of low-mass stars in Trumpler 16 with Chandra, Online Material p 23

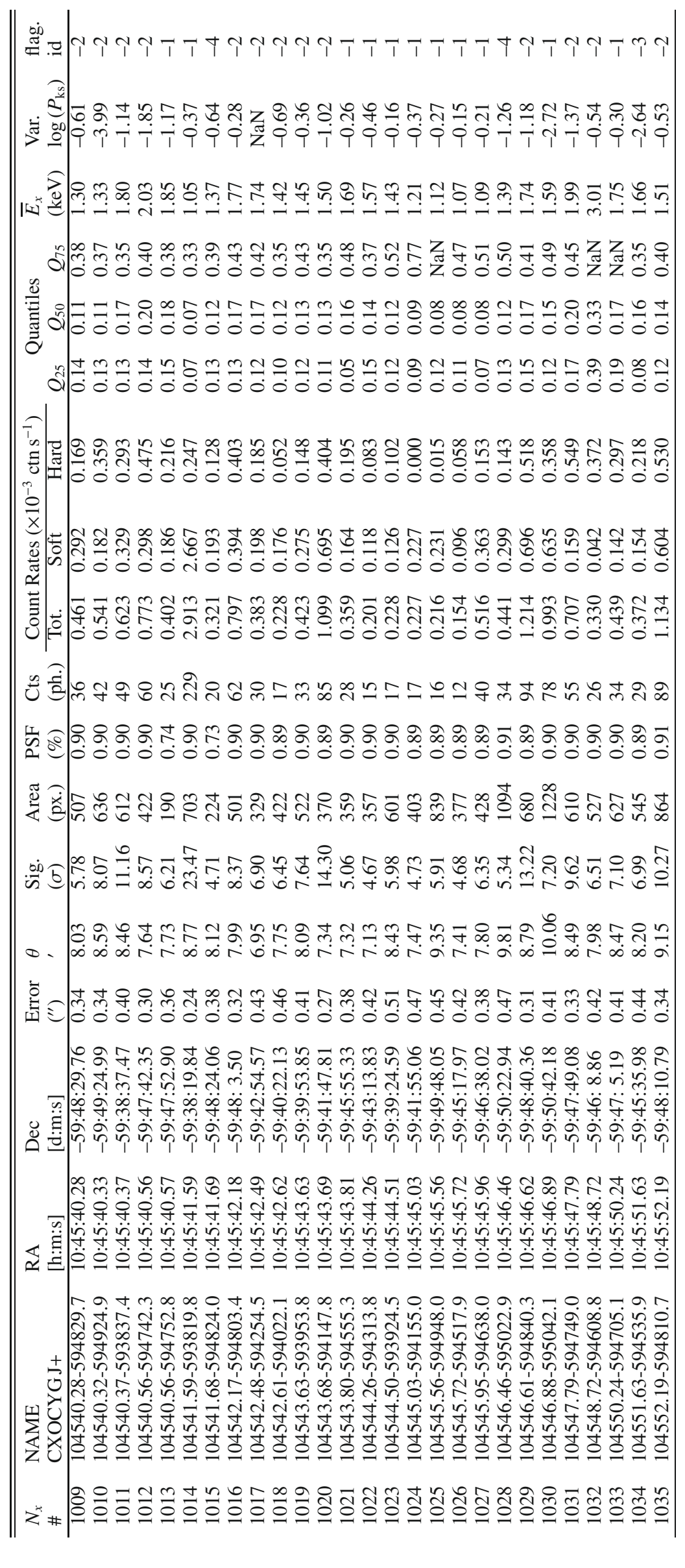


Table 2. Near-IR counterparts of Trumpler 16 X-ray sources.

\begin{tabular}{|c|c|c|c|c|c|c|c|c|c|c|}
\hline$N_{x}$ & 2MASS J+ & Off." & $J$ mag & $H$ mag & $K_{\mathrm{s}} \mathrm{mag}$ & Ph.Q & Cont. & $A_{\mathrm{v}}$ & Mass & ID.flags \\
\hline 1 & $10433859-5938306$ & 2.33 & $14.57 \pm 0.03$ & $13.21 \pm 0.04$ & $12.63 \pm 0.03$ & AAA & 000 & 7.93 & 2.05 & \\
\hline 2 & -------- & -- & ----- & ----- & ----- & -- & -- & -- & -- & \\
\hline 3 & $10434145-5942245$ & 0.30 & $14.18 \pm 0.03$ & $13.20 \pm 0.02$ & $12.82 \pm 0.02$ & AAA & 000 & 4.59 & 2.24 & \\
\hline 4 & $10434126-5941002$ & 2.62 & $15.47 \pm * * * *$ & $15.31 \pm 0.14$ & $14.60 \pm 0.14$ & UBB & 000 & $\mathrm{NaN}$ & $\mathrm{NaN}$ & \\
\hline 5 & $10434295-5944080$ & 1.77 & $15.63 \pm 0.08$ & $14.31 \pm 0.06$ & $13.27 \pm 0.04$ & AAA & 000 & 15.27 & 1.25 & K-excess \\
\hline 6 & -------- & -- & ----- & ----- & ----- & -- & -- & -- & -- & \\
\hline 7 & $10434401-5948177$ & 0.76 & $8.70 \pm 0.02$ & $8.51 \pm 0.04$ & $8.47 \pm 0.02$ & AAA & 000 & 1.38 & 19.15 & $09.5 \mathrm{~V}$ \\
\hline 8 & $10434538-5939468$ & 1.92 & $15.90 \pm * * * *$ & $14.88 \pm * * * *$ & $14.90 \pm 0.16$ & UUC & 000 & $\mathrm{NaN}$ & $\mathrm{NaN}$ & \\
\hline 9 & $10434536-5938471$ & 0.36 & $16.02 \pm 0.10$ & $14.04 \pm 0.04$ & $13.02 \pm 0.04$ & AAA & 000 & 14.90 & 1.01 & \\
\hline 10 & $10434525-5941567$ & 2.61 & $14.37 \pm 0.06$ & $12.99 \pm 0.05$ & $12.18 \pm 0.04$ & AAA & 000 & 12.05 & 2.16 & K-excess \\
\hline 11 & $10434659-5949292$ & 1.68 & $13.69 \pm 0.05$ & $12.68 \pm 0.05$ & $12.25 \pm 0.04$ & AEA & $\mathrm{c} 0 \mathrm{c}$ & $\mathrm{NaN}$ & 2.38 & \\
\hline 12 & $10434809-5949246$ & 0.50 & $13.51 \pm * * * *$ & $13.09 \pm 0.07$ & $12.85 \pm 0.05$ & UAA & $0 \mathrm{cc}$ & $\mathrm{NaN}$ & $\mathrm{NaN}$ & \\
\hline 13 & $10434937-5944549$ & 1.45 & $12.67 \pm 0.02$ & $12.20 \pm 0.03$ & $12.06 \pm 0.03$ & AAA & 000 & 0.82 & 4.89 & \\
\hline 14 & $10435007-5945530$ & 0.59 & $15.56 \pm 0.05$ & $14.59 \pm 0.03$ & $14.19 \pm 0.07$ & AAA & 000 & 4.31 & 1.30 & \\
\hline 15 & $10435085-5937437$ & 1.28 & $14.86 \pm 0.05$ & $13.74 \pm 0.05$ & $13.40 \pm 0.05$ & AAA & 000 & 3.76 & 1.86 & \\
\hline 16 & $10435088-5950307$ & 0.47 & $12.20 \pm 0.02$ & $11.99 \pm 0.03$ & $11.88 \pm 0.02$ & AAA & 000 & 0.28 & 6.22 & \\
\hline 17 & $10435123-5940243$ & 1.06 & $14.60 \pm 0.05$ & $13.38 \pm 0.04$ & $12.88 \pm 0.04$ & AAA & 000 & 6.57 & 2.03 & \\
\hline 18 & $10435132-5945239$ & 2.64 & $15.54 \pm 0.08$ & $14.06 \pm * * * *$ & $13.55 \pm * * * *$ & AUU & cpp & $\mathrm{NaN}$ & 1.31 & \\
\hline 19 & $10435191-5940353$ & 0.40 & $16.49 \pm 0.16$ & $15.15 \pm 0.09$ & $14.63 \pm 0.11$ & CAA & 000 & $\mathrm{NaN}$ & 0.70 & \\
\hline 20 & $10435186-5948017$ & 2.02 & $14.49 \pm 0.05$ & $13.46 \pm 0.06$ & $12.95 \pm 0.05$ & AAA & 000 & 6.60 & 2.09 & \\
\hline 21 & $10435223-5941574$ & 0.29 & $14.83 \pm 0.05$ & $14.46 \pm 0.08$ & $14.35 \pm 0.11$ & AAA & 000 & $\mathrm{NaN}$ & 1.88 & \\
\hline 22 & $10435230-5939222$ & 1.87 & $13.03 \pm 0.04$ & $11.90 \pm 0.06$ & $11.20 \pm 0.04$ & AEE & 000 & $\mathrm{NaN}$ & 3.55 & Mass-deg. \\
\hline 23 & $10435408-5941463$ & 1.19 & $15.40 \pm 0.07$ & $14.24 \pm 0.05$ & $13.79 \pm 0.05$ & AAA & 000 & 5.47 & 1.40 & \\
\hline 24 & $10435419-5938073$ & 2.13 & $13.03 \pm * * * *$ & $13.70 \pm 0.06$ & $13.53 \pm 0.07$ & UAA & $0 \mathrm{cc}$ & $\mathrm{NaN}$ & $\mathrm{NaN}$ & \\
\hline 25 & $10435501-5936242$ & 0.75 & $11.64 \pm 0.02$ & $11.48 \pm 0.03$ & $11.37 \pm 0.03$ & AAA & 000 & 0.42 & 8.28 & \\
\hline 26 & $10435505-5947505$ & 0.70 & $14.18 \pm 0.03$ & $13.81 \pm 0.04$ & $13.70 \pm 0.06$ & AAA & 000 & $\mathrm{NaN}$ & 2.24 & \\
\hline 27 & $10435545-5942531$ & 0.50 & $14.93 \pm 0.05$ & $14.11 \pm 0.05$ & $13.84 \pm 0.06$ & AAA & 000 & 2.37 & 1.81 & \\
\hline 28 & $10435557-5949226$ & 0.39 & $13.44 \pm 0.03$ & $12.65 \pm 0.03$ & $12.24 \pm 0.03$ & AAA & 000 & 5.08 & 2.45 & \\
\hline 29 & $10435606-5949351$ & 1.97 & $14.53 \pm 0.05$ & $13.83 \pm 0.05$ & $13.60 \pm 0.06$ & AAA & 000 & 1.87 & 2.07 & \\
\hline 30 & $10435684-5942364$ & 0.45 & $14.70 \pm 0.05$ & $13.57 \pm 0.03$ & $12.88 \pm 0.04$ & AAA & 000 & 9.63 & 1.97 & K-excess \\
\hline 31 & $10435797-5949022$ & 1.04 & $15.45 \pm * * * *$ & $14.71 \pm 0.06$ & $14.50 \pm 0.11$ & UAB & 000 & $\mathrm{NaN}$ & $\mathrm{NaN}$ & \\
\hline 32 & -------- & -- & ----- & ----- & ----- & -- & -- & -- & -- & \\
\hline 33 & $10435930-5936527$ & 0.17 & $15.52 \pm 0.09$ & $14.37 \pm 0.06$ & $13.91 \pm 0.07$ & AAA & 000 & 5.51 & 1.33 & \\
\hline 34 & $10440004-5936400$ & 2.62 & $14.34 \pm 0.03$ & $13.49 \pm 0.02$ & $13.17 \pm 0.03$ & AAA & spp & 3.48 & 2.18 & \\
\hline 35 & ------- & -- & ----- & ----- & ----- & -- & -- & -- & -- & \\
\hline 36 & $10440100-5944098$ & 0.24 & $13.54 \pm 0.03$ & $12.26 \pm 0.02$ & $11.43 \pm 0.02$ & AAA & 000 & $\mathrm{NaN}$ & 2.42 & K-excess \\
\hline 37 & -------- & -- & ----- & ----- & ----- & -- & -- & -- & -- & \\
\hline 38 & -------- & -- & ----- & ----- & ----- & -- & -- & -- & -- & \\
\hline 39 & $10440164-5944580$ & 0.42 & $13.92 \pm 0.03$ & $13.33 \pm 0.04$ & $12.89 \pm 0.04$ & AAA & $\mathrm{c} 00$ & 5.53 & 2.31 & K-excess \\
\hline 40 & $10440206-5945114$ & 0.39 & $15.09 \pm 0.06$ & $14.06 \pm 0.05$ & $13.43 \pm 0.05$ & AAA & 000 & 8.56 & 1.66 & K-excess \\
\hline 41 & $10440209-5937522$ & 1.34 & $12.06 \pm 0.02$ & $11.22 \pm 0.02$ & $10.57 \pm 0.02$ & AAA & 000 & $\mathrm{NaN}$ & 6.71 & K-excess \\
\hline 42 & $10440234-5948257$ & 1.01 & $15.03 \pm 0.05$ & $14.02 \pm 0.04$ & $13.65 \pm 0.05$ & AAA & 000 & 4.03 & 1.72 & \\
\hline 43 & $10440277-5939464$ & 0.19 & $10.96 \pm 0.02$ & $10.36 \pm 0.02$ & $10.19 \pm 0.02$ & AAA & 000 & 3.48 & 10.79 & \\
\hline 44 & -------- & -- & ----- & ----- & ----- & -- & -- & -- & -- & \\
\hline 45 & -------- & -- & ----- & ----- & ----- & -- & -- & -- & -- & \\
\hline 46 & $10440355-5937000$ & 0.65 & $14.54 \pm 0.05$ & $13.68 \pm 0.03$ & $13.45 \pm 0.05$ & AAA & 000 & 1.95 & 2.07 & \\
\hline 47 & -------- & -- & ----- & ----- & ----- & -- & -- & -- & -- & \\
\hline 48 & $10440402-5944142$ & 0.81 & $15.81 \pm * * * *$ & $14.78 \pm 0.07$ & $14.39 \pm 0.12$ & UAB & $0 \mathrm{cc}$ & $\mathrm{NaN}$ & $\mathrm{NaN}$ & \\
\hline 49 & -------- & -- & ----- & ----- & ----- & -- & -- & -- & -- & \\
\hline 50 & $10440533-5945433$ & 0.50 & $15.94 \pm 0.10$ & $15.11 \pm 0.09$ & $15.98 \pm * * * *$ & AAU & 000 & $\mathrm{NaN}$ & 1.06 & \\
\hline 51 & $10440579-5943543$ & 1.14 & $15.62 \pm 0.10$ & $14.38 \pm 0.06$ & $13.86 \pm 0.07$ & AAA & $\mathrm{c} 00$ & 6.50 & 1.26 & \\
\hline 52 & $10440583-5935116$ & 1.27 & $10.16 \pm 0.02$ & $9.97 \pm 0.02$ & $9.92 \pm 0.02$ & AAA & 000 & 1.63 & 13.76 & B1V \\
\hline 53 & $10440588-5939087$ & 0.75 & $14.87 \pm 0.07$ & $14.14 \pm 0.07$ & $13.64 \pm 0.07$ & AAA & 000 & 6.25 & 1.85 & K-excess \\
\hline 54 & $10440591-5946488$ & 0.86 & $14.92 \pm 0.11$ & $14.16 \pm 0.08$ & $13.76 \pm 0.16$ & $\mathrm{AAB}$ & $\mathrm{ccc}$ & $\mathrm{NaN}$ & 1.81 & \\
\hline 55 & ------- & -- & ----- & ----- & ----- & -- & -- & -- & -- & \\
\hline 56 & $10440683-5936116$ & 0.44 & $11.73 \pm 0.02$ & $11.33 \pm 0.02$ & $11.14 \pm 0.02$ & AAA & 000 & 1.99 & 7.94 & \\
\hline 57 & $10440695-5941469$ & 0.21 & $15.34 \pm * * * *$ & $14.20 \pm * * * *$ & $13.81 \pm 0.07$ & UUA & $00 \mathrm{c}$ & $\mathrm{NaN}$ & $\mathrm{NaN}$ & \\
\hline 58 & $10440727-5948461$ & 1.09 & $11.56 \pm 0.02$ & $10.78 \pm 0.02$ & $10.61 \pm 0.02$ & AAA & 000 & $\mathrm{NaN}$ & 8.59 & \\
\hline 59 & ------- & -- & ----- & ----- & ----- & -- & -- & -- & -- & \\
\hline 60 & -------- & -- & ----- & ----- & ----- & -- & -- & -- & -- & \\
\hline 61 & $10440804-5945222$ & 0.57 & $14.62 \pm 0.05$ & $13.82 \pm 0.04$ & $13.32 \pm 0.05$ & AAA & 000 & 6.46 & 2.02 & K-excess \\
\hline 62 & $10440820-5940373$ & 0.09 & $14.76 \pm 0.07$ & $14.00 \pm 0.06$ & $13.67 \pm 0.07$ & AAA & $\mathrm{ccc}$ & 3.55 & 1.93 & \\
\hline 63 & $10440871-5936104$ & 0.49 & $13.05 \pm 0.03$ & $12.49 \pm 0.03$ & $12.31 \pm 0.04$ & AAA & $\mathrm{ccc}$ & 1.52 & 3.55 & Mass-deg. \\
\hline 64 & $10440861-5938281$ & 2.43 & $16.62 \pm 0.22$ & $15.31 \pm 0.11$ & $14.73 \pm * * * *$ & DBU & $\operatorname{cc} 0$ & $\mathrm{NaN}$ & 0.65 & \\
\hline 65 & $10440910-5945388$ & 0.12 & $15.45 \pm 0.07$ & $14.47 \pm 0.05$ & $13.99 \pm 0.07$ & AAA & $\mathrm{ccc}$ & 5.84 & 1.37 & \\
\hline 66 & ------- & -- & ----- & ----- & ----- & -- & -- & -- & -- & \\
\hline
\end{tabular}


Table 2. continued.

\begin{tabular}{|c|c|c|c|c|c|c|c|c|c|c|}
\hline$N_{x}$ & 2MASS J+ & Off." & $J$ mag & $H$ mag & $K_{\mathrm{s}} \mathrm{mag}$ & Ph.Q & Cont. & $A_{\mathrm{v}}$ & Mass & ID.flags \\
\hline 67 & $10440958-5951031$ & 1.07 & $15.48 \pm * * * *$ & $14.86 \pm 0.10$ & $14.94 \pm 0.14$ & UAB & $0 \mathrm{cc}$ & $\overline{\mathrm{NaN}}$ & $\mathrm{NaN}$ & \\
\hline 68 & $10440976-5943383$ & 1.26 & $12.95 \pm 0.03$ & $12.56 \pm 0.02$ & $12.33 \pm 0.03$ & AAA & $00 \mathrm{c}$ & 2.24 & 3.55 & Mass-deg. \\
\hline 69 & $10440976-5944480$ & 0.60 & $12.62 \pm 0.03$ & $11.98 \pm 0.03$ & $11.75 \pm 0.03$ & AAA & $\operatorname{ccc}$ & 2.36 & 4.98 & \\
\hline 70 & $10441017-5935514$ & 0.88 & $14.57 \pm 0.05$ & $13.78 \pm 0.05$ & $13.48 \pm 0.06$ & AAA & $0 \mathrm{cc}$ & 2.99 & 2.05 & \\
\hline 71 & $10441031-5948171$ & 0.65 & $14.80 \pm 0.04$ & $14.15 \pm * * * *$ & $14.19 \pm * * * *$ & AUU & $\mathrm{c} 00$ & $\mathrm{NaN}$ & 1.90 & \\
\hline 72 & $10441035-5939566$ & 1.71 & $15.41 \pm 0.08$ & $14.75 \pm 0.07$ & $14.42 \pm 0.11$ & AAB & $\mathrm{ccc}$ & $\mathrm{NaN}$ & 1.39 & \\
\hline 73 & $10441037-5943534$ & 1.12 & $14.22 \pm * * * *$ & $13.91 \pm 0.06$ & $13.26 \pm 0.07$ & UAA & $00 \mathrm{c}$ & $\mathrm{NaN}$ & $\mathrm{NaN}$ & \\
\hline 74 & $10441038-5943111$ & 0.30 & $6.26 \pm 0.01$ & $5.97 \pm 0.02$ & $5.72 \pm 0.01$ & AAA & ddd & $\mathrm{NaN}$ & 28.21 & WN6ha + O4f \\
\hline 75 & -------- & -- & ----- & ----- & ----- & -- & -- & -- & -- & \\
\hline 76 & $10441079-5940135$ & 0.60 & $14.65 \pm 0.05$ & $13.81 \pm 0.05$ & $13.54 \pm 0.06$ & AAA & 000 & 2.56 & 2.00 & \\
\hline 77 & -------- & -- & ----- & ----- & ----- & -- & -- & -- & -- & \\
\hline 78 & $10441134-5945333$ & 0.29 & $16.03 \pm 0.13$ & $14.80 \pm 0.08$ & $14.38 \pm 0.10$ & BAA & 000 & $\mathrm{NaN}$ & 1.01 & \\
\hline 79 & $10441161-5939168$ & 0.45 & $14.60 \pm 0.05$ & $13.75 \pm 0.05$ & $13.22 \pm 0.05$ & AAA & $\mathrm{c} 00$ & 6.98 & 2.03 & K-excess \\
\hline 80 & $10441184-5942233$ & 0.31 & $15.35 \pm 0.07$ & $14.19 \pm 0.05$ & $12.96 \pm * * * *$ & AAU & $\operatorname{cc} 0$ & $\mathrm{NaN}$ & 1.43 & \\
\hline 81 & $10441186-5941433$ & 0.50 & $15.32 \pm 0.07$ & $14.64 \pm 0.06$ & $14.58 \pm 0.12$ & AAB & 000 & $\mathrm{NaN}$ & 1.46 & \\
\hline 82 & -------- & -- & ----- & ----- & ----- & -- & -- & -- & -- & \\
\hline 83 & $10441187-5944146$ & 0.38 & $15.48 \pm 0.08$ & $14.49 \pm 0.05$ & $14.06 \pm 0.08$ & AAA & $\mathrm{ccc}$ & 4.93 & 1.35 & \\
\hline 84 & $10441243-5942127$ & 0.63 & $16.28 \pm * * * *$ & $15.10 \pm 0.11$ & $14.46 \pm * * * *$ & UBU & 000 & $\mathrm{NaN}$ & $\mathrm{NaN}$ & \\
\hline 85 & $10441249-5943512$ & 0.57 & $15.57 \pm 0.13$ & $14.63 \pm 0.11$ & $14.16 \pm 0.12$ & BBB & $\mathrm{ccc}$ & $\mathrm{NaN}$ & 1.29 & \\
\hline 86 & $10441282-5944187$ & 0.46 & $15.35 \pm 0.08$ & $14.40 \pm 0.05$ & $13.86 \pm 0.07$ & AAA & $\mathrm{ccc}$ & 6.74 & 1.44 & \\
\hline 87 & -------- & -- & ----- & ----- & ----- & -- & -- & -- & -- & \\
\hline 88 & -------- & -- & ----- & ----- & ----- & -- & -- & -- & -- & \\
\hline 89 & $10441319-5943103$ & 0.34 & $7.84 \pm 0.01$ & $7.38 \pm 0.04$ & $7.06 \pm 0.02$ & AAA & ddd & 6.01 & 22.36 & O4If \\
\hline 90 & -------- & -- & ----- & ----- & ----- & -- & -- & -- & -- & \\
\hline 91 & $10441389-5942404$ & 0.58 & $15.83 \pm 0.15$ & $15.07 \pm 0.12$ & $14.53 \pm 0.14$ & BBB & $\mathrm{ccc}$ & $\mathrm{NaN}$ & 1.13 & \\
\hline 92 & $10441385-5942249$ & 0.23 & $14.38 \pm 0.05$ & $13.55 \pm 0.04$ & $13.34 \pm 0.05$ & AAA & 000 & 1.66 & 2.16 & \\
\hline 93 & -------- & -- & ----- & ----- & ----- & -- & -- & -- & -- & \\
\hline 94 & -------- & -- & ----- & ----- & ----- & -- & -- & -- & -- & \\
\hline 95 & -------- & -- & ----- & ----- & ----- & -- & -- & -- & -- & \\
\hline 96 & $10441525-5944475$ & 1.85 & $15.33 \pm 0.08$ & $14.52 \pm 0.06$ & $13.91 \pm * * * *$ & AAU & $\operatorname{cc0}$ & $\mathrm{NaN}$ & 1.45 & \\
\hline 97 & -------- & -- & ----- & ----- & ----- & -- & -- & -- & -- & \\
\hline 98 & -------- & -- & ----- & ----- & ----- & -- & -- & -- & -- & \\
\hline 99 & -------- & -- & ----- & ----- & ----- & -- & -- & -- & -- & \\
\hline 100 & $10441574-5938153$ & 1.68 & $15.28 \pm 0.10$ & $14.37 \pm 0.08$ & $13.81 \pm * * * *$ & AAU & $\operatorname{cc} 0$ & $\mathrm{NaN}$ & 1.49 & \\
\hline 101 & $10441594-5940236$ & 0.60 & $14.02 \pm 0.03$ & $13.15 \pm 0.03$ & $12.68 \pm 0.03$ & AAA & 000 & 6.10 & 2.28 & \\
\hline 102 & $10441629-5944215$ & 0.43 & $14.62 \pm 0.06$ & $13.97 \pm 0.06$ & $13.60 \pm 0.06$ & AAA & $\mathrm{c} 0 \mathrm{c}$ & 4.24 & 2.02 & \\
\hline 103 & -------- & -- & ----- & ----- & ----- & -- & -- & -- & -- & \\
\hline 104 & $10441648-5935100$ & 0.78 & $13.26 \pm 0.03$ & $12.60 \pm 0.03$ & $12.34 \pm 0.03$ & AAA & 000 & 2.66 & 2.50 & \\
\hline 105 & $10441711-5941568$ & 2.35 & $13.81 \pm 0.03$ & $12.97 \pm 0.03$ & $12.70 \pm 0.03$ & AAA & 000 & 2.94 & 2.34 & \\
\hline 106 & $10441742-5945475$ & 0.26 & $15.60 \pm * * * *$ & $14.40 \pm * * * *$ & $13.72 \pm 0.06$ & UUA & $00 \mathrm{c}$ & $\mathrm{NaN}$ & $\mathrm{NaN}$ & \\
\hline 107 & $10441752-5943331$ & 0.19 & $14.78 \pm 0.06$ & $13.99 \pm 0.05$ & $13.90 \pm 0.08$ & AAA & $\mathrm{ccc}$ & $\mathrm{NaN}$ & 1.92 & \\
\hline 108 & -------- & -- & ----- & ----- & ----- & -- & -- & -- & -- & \\
\hline 109 & -------- & -- & ----- & ----- & ----- & -- & -- & -- & -- & \\
\hline 110 & $10441770-5942359$ & 0.33 & $14.64 \pm 0.06$ & $13.94 \pm 0.06$ & $13.61 \pm 0.07$ & AAA & $\mathrm{cc} 0$ & 3.55 & 2.01 & \\
\hline 111 & $10441794-5943570$ & 0.17 & $14.84 \pm 0.05$ & $13.96 \pm 0.04$ & $13.57 \pm 0.06$ & AAA & $\mathrm{ccc}$ & 4.48 & 1.87 & \\
\hline 112 & $10441835-5943474$ & 1.09 & $15.80 \pm 0.14$ & $14.81 \pm 0.10$ & $14.19 \pm 0.10$ & BAA & pec & $\mathrm{NaN}$ & 1.15 & \\
\hline 113 & -------- & -- & ----- & ----- & ----- & -- & -- & -- & -- & \\
\hline 114 & $10441944-5939503$ & 0.44 & $13.79 \pm 0.01$ & $12.90 \pm 0.02$ & $12.26 \pm 0.01$ & AAA & 000 & 8.90 & 2.35 & K-excess \\
\hline 115 & $10441951-5943518$ & 0.74 & $14.75 \pm 0.05$ & $13.95 \pm 0.05$ & $13.69 \pm 0.06$ & AAA & $\mathrm{ccc}$ & 2.27 & 1.94 & \\
\hline 116 & -------- & -- & ----- & ----- & ----- & -- & -- & -- & -- & \\
\hline 117 & -------- & -- & ----- & ----- & ----- & -- & -- & -- & -- & \\
\hline 118 & -------- & -- & ----- & ----- & ----- & -- & -- & -- & -- & \\
\hline 119 & $10442013-5943396$ & 0.57 & $14.91 \pm 0.11$ & $13.52 \pm * * * *$ & $13.17 \pm * * * *$ & AUU & $\mathrm{c} 00$ & $\mathrm{NaN}$ & 1.82 & \\
\hline 120 & $10442019-5938264$ & 0.21 & $15.08 \pm 0.10$ & $14.28 \pm 0.09$ & $14.13 \pm 0.10$ & AAA & $\mathrm{ccc}$ & $\mathrm{NaN}$ & 1.67 & \\
\hline 121 & $10442016-5942296$ & 0.33 & $14.03 \pm 0.04$ & $13.24 \pm 0.04$ & $12.86 \pm 0.05$ & AAA & 000 & 4.63 & 2.28 & \\
\hline 122 & $10442040-5944155$ & 0.43 & $16.00 \pm 0.14$ & $15.09 \pm 0.11$ & $15.05 \pm * * * *$ & BBU & $\operatorname{cc} 0$ & $\mathrm{NaN}$ & 1.02 & \\
\hline 123 & -------- & -- & ----- & ----- & ----- & -- & -- & -- & -- & \\
\hline 124 & $10442056-5950282$ & 0.26 & $14.46 \pm 0.03$ & $13.36 \pm 0.02$ & $12.85 \pm 0.03$ & AAA & 000 & 6.70 & 2.11 & \\
\hline 125 & $10442096-5945407$ & 1.28 & $15.59 \pm * * * *$ & $14.23 \pm 0.05$ & $13.71 \pm 0.06$ & UAA & $0 \mathrm{cc}$ & $\mathrm{NaN}$ & $\mathrm{NaN}$ & \\
\hline 126 & $10442104-5944198$ & 0.33 & $14.63 \pm 0.05$ & $13.98 \pm 0.05$ & $13.71 \pm 0.07$ & AAA & $\mathrm{ccc}$ & 2.52 & 2.01 & \\
\hline 127 & -------- & -- & ----- & ----- & ----- & -- & -- & -- & -- & \\
\hline 128 & -------- & -- & ----- & ----- & ----- & -- & -- & -- & -- & \\
\hline 129 & -------- & -- & ----- & ----- & ----- & -- & -- & -- & -- & \\
\hline 130 & -------- & -- & ----- & ----- & ----- & -- & -- & -- & -- & \\
\hline 131 & $10442202-5940045$ & 0.11 & $15.42 \pm 0.09$ & $14.60 \pm 0.07$ & $14.26 \pm 0.10$ & AAA & 000 & 3.07 & 1.39 & \\
\hline 132 & -------- & -- & ----- & ----- & ----- & -- & -- & -- & -- & \\
\hline
\end{tabular}


J. F. Albacete-Colombo et al.: An X-ray survey of low-mass stars in Trumpler 16 with Chandra, Online Material p 26

Table 2. continued.

\begin{tabular}{|c|c|c|c|c|c|c|c|c|c|c|}
\hline$N_{x}$ & 2 MASS J+ & Off." & $J \mathrm{mag}$ & $H$ mag & $K_{\mathrm{s}} \mathrm{mag}$ & Ph.Q & Cont. & $A_{\mathrm{v}}$ & Mass & ID.flags \\
\hline 133 & $10442219-5950575$ & 0.57 & $12.40 \pm 0.02$ & $11.84 \pm 0.02$ & $11.62 \pm 0.02$ & $\overline{\mathrm{AAA}}$ & 000 & 2.07 & 5.59 & \\
\hline 134 & $10442236-5943086$ & 0.09 & $15.03 \pm 0.07$ & $14.04 \pm * * * *$ & $13.83 \pm * * * *$ & AUU & $\mathrm{c} 00$ & $\mathrm{NaN}$ & 1.72 & \\
\hline 135 & $10442256-5943415$ & 0.27 & $16.87 \pm * * * *$ & $15.52 \pm * * * *$ & $14.68 \pm 0.13$ & UUB & $00 \mathrm{c}$ & $\mathrm{NaN}$ & $\mathrm{NaN}$ & \\
\hline 136 & $10442251-5939258$ & 0.82 & $10.66 \pm 0.02$ & $10.55 \pm 0.02$ & $10.51 \pm 0.02$ & AAA & ss0 & 1.21 & 11.90 & B $1.5 \mathrm{~V}$ \\
\hline 137 & $10442247-5944034$ & 0.22 & $15.75 \pm 0.10$ & $14.69 \pm 0.10$ & $14.36 \pm 0.11$ & $\mathrm{AAB}$ & $\mathrm{ccc}$ & $\mathrm{NaN}$ & 1.18 & \\
\hline 138 & $10442244-5938236$ & 0.50 & $14.90 \pm * * * *$ & $14.30 \pm 0.06$ & $13.54 \pm 0.05$ & UAA & $00 \mathrm{c}$ & $\mathrm{NaN}$ & $\mathrm{NaN}$ & \\
\hline 139 & -------- & -- & ----- & ----- & ----- & -- & -- & -- & -- & \\
\hline 140 & -------- & -- & ----- & ----- & ----- & -- & -- & -- & -- & \\
\hline 141 & $10442279-5944323$ & 1.34 & $14.10 \pm 0.03$ & $13.29 \pm 0.02$ & $12.94 \pm 0.03$ & AAA & 000 & 4.18 & 2.26 & \\
\hline 142 & $10442285-5938013$ & 0.12 & $14.84 \pm 0.07$ & $13.87 \pm 0.05$ & $13.28 \pm 0.06$ & AAA & $\operatorname{cc} 0$ & 8.00 & 1.87 & K-excess \\
\hline 143 & $10442308-5943063$ & 2.03 & $14.94 \pm 0.08$ & $14.24 \pm 0.08$ & $13.98 \pm 0.09$ & AAA & $\mathrm{ccc}$ & 2.10 & 1.79 & \\
\hline 144 & $10442312-5941552$ & 0.14 & $14.75 \pm * * * *$ & $13.65 \pm * * * *$ & $13.14 \pm 0.05$ & UUA & $00 \mathrm{c}$ & $\mathrm{NaN}$ & $\mathrm{NaN}$ & \\
\hline 145 & $10442318-5942070$ & 0.21 & $14.83 \pm * * * *$ & $14.28 \pm 0.09$ & $13.97 \pm 0.13$ & UAB & $0 \mathrm{cc}$ & $\mathrm{NaN}$ & $\mathrm{NaN}$ & \\
\hline 146 & $10442356-5939414$ & 0.63 & $13.72 \pm 0.04$ & $13.14 \pm 0.03$ & $12.83 \pm 0.05$ & AAA & 000 & 3.58 & 2.37 & \\
\hline 147 & $10442362-5941151$ & 0.14 & $13.69 \pm 0.03$ & $12.90 \pm 0.03$ & $12.63 \pm 0.03$ & AAA & 000 & 2.82 & 2.38 & \\
\hline 148 & -------- & -- & ----- & ----- & ----- & -- & -- & -- & -- & \\
\hline 149 & -------- & -- & ----- & ----- & ----- & -- & -- & -- & -- & \\
\hline 150 & $10442407-5944222$ & 0.69 & $14.76 \pm 0.06$ & $13.77 \pm 0.06$ & $13.18 \pm 0.05$ & AAA & $\mathrm{ccc}$ & 7.99 & 1.93 & K-excess \\
\hline 151 & $10442404-5940559$ & 1.00 & $13.76 \pm 0.03$ & $13.08 \pm 0.03$ & $12.93 \pm 0.04$ & AAA & $\mathrm{ccc}$ & 0.77 & 2.35 & \\
\hline 152 & $10442417-5937280$ & 0.35 & $15.44 \pm 0.06$ & $14.48 \pm 0.05$ & $14.18 \pm 0.08$ & AAA & 000 & 2.32 & 1.37 & \\
\hline 153 & -------- & -- & ----- & ----- & ----- & -- & -- & -- & -- & \\
\hline 154 & -------- & -- & ----- & ----- & ----- & -- & -- & -- & -- & \\
\hline 155 & $10442482-5939596$ & 0.23 & $14.64 \pm 0.05$ & $13.76 \pm 0.06$ & $13.22 \pm 0.05$ & AAA & $\operatorname{cc} 0$ & 7.14 & 2.01 & K-excess \\
\hline 156 & $10442489-5937026$ & 0.22 & $14.27 \pm 0.03$ & $13.40 \pm 0.03$ & $13.15 \pm 0.04$ & AAA & $00 \mathrm{~s}$ & 2.24 & 2.21 & \\
\hline 157 & $10442498-5936478$ & 0.16 & $14.76 \pm 0.05$ & $14.02 \pm 0.05$ & $13.79 \pm 0.07$ & AAA & 000 & 1.78 & 1.93 & \\
\hline 158 & $10442522-5936215$ & 0.29 & $13.27 \pm 0.03$ & $12.65 \pm 0.03$ & $12.46 \pm 0.04$ & AAA & $\mathrm{ccc}$ & 1.67 & 2.50 & \\
\hline 159 & $10442538-5941114$ & 0.56 & $14.59 \pm 0.06$ & $14.00 \pm 0.06$ & $13.79 \pm 0.07$ & AAA & $\mathrm{ccc}$ & 1.37 & 2.04 & \\
\hline 160 & -------- & -- & ----- & ----- & ----- & -- & -- & -- & -- & \\
\hline 161 & $10442564-5943534$ & 0.31 & $14.04 \pm 0.04$ & $13.29 \pm 0.05$ & $13.06 \pm 0.06$ & AAA & 000 & 2.07 & 2.27 & \\
\hline 162 & -------- & -- & ----- & ----- & ----- & -- & -- & -- & -- & \\
\hline 163 & -------- & -- & ----- & ----- & ----- & -- & -- & -- & -- & \\
\hline 164 & $10442564-5943534$ & 1.14 & $14.04 \pm 0.04$ & $13.29 \pm 0.05$ & $13.06 \pm 0.06$ & AAA & 000 & 2.07 & 2.27 & \\
\hline 165 & $10442567-5939238$ & 0.77 & $14.95 \pm 0.04$ & $13.96 \pm 0.03$ & $13.50 \pm 0.04$ & AAE & 000 & $\mathrm{NaN}$ & 1.78 & \\
\hline 166 & $10442568-5943287$ & 0.70 & $14.19 \pm * * * *$ & $13.67 \pm 0.06$ & $13.38 \pm 0.08$ & UAA & $0 \mathrm{cc}$ & $\mathrm{NaN}$ & $\mathrm{NaN}$ & \\
\hline 167 & -------- & -- & ----- & ----- & ----- & -- & -- & -- & -- & \\
\hline 168 & -------- & -- & ----- & ----- & ----- & -- & -- & -- & -- & \\
\hline 169 & $10442597-5944267$ & 1.21 & $14.89 \pm 0.07$ & $14.02 \pm 0.06$ & $13.52 \pm 0.07$ & AAA & $\mathrm{c} 00$ & 6.31 & 1.84 & \\
\hline 170 & $10442628-5942412$ & 1.49 & $14.15 \pm 0.03$ & $13.43 \pm 0.02$ & $13.15 \pm 0.04$ & AAA & 000 & 2.84 & 2.24 & \\
\hline 171 & -------- & -- & ----- & ----- & ----- & -- & -- & -- & -- & \\
\hline 172 & $10442630-5937403$ & 0.27 & $15.43 \pm 0.07$ & $14.36 \pm 0.05$ & $13.98 \pm 0.07$ & AAA & 000 & 4.18 & 1.38 & \\
\hline 173 & $10442685-5945412$ & 0.68 & $14.17 \pm 0.05$ & $13.71 \pm 0.07$ & $13.40 \pm 0.05$ & AAA & $\mathrm{ccc}$ & 3.22 & 2.24 & \\
\hline 174 & $10442704-5944389$ & 0.97 & $13.48 \pm 0.04$ & $12.79 \pm 0.03$ & $12.55 \pm 0.03$ & AAA & 000 & 2.30 & 2.44 & \\
\hline 175 & $10442729-5940357$ & 0.21 & $15.23 \pm 0.07$ & $14.47 \pm 0.06$ & $14.23 \pm 0.09$ & AAA & 000 & 0.98 & 1.53 & \\
\hline 176 & -------- & -- & ----- & ----- & ----- & -- & -- & -- & -- & \\
\hline 177 & -------- & -- & ----- & ----- & ----- & -- & -- & -- & -- & \\
\hline 178 & -------- & -- & ----- & ----- & ----- & -- & -- & -- & -- & \\
\hline 179 & $10442781-5945213$ & 0.34 & $8.31 \pm 0.02$ & $7.83 \pm 0.04$ & $7.74 \pm 0.03$ & AAA & 000 & 2.33 & 20.63 & \\
\hline 180 & $10442786-5937477$ & 0.23 & $14.59 \pm 0.04$ & $13.64 \pm 0.05$ & $13.28 \pm 0.05$ & AAA & $\mathrm{ccc}$ & 4.08 & 2.04 & \\
\hline 181 & -------- & -- & ----- & ----- & ----- & -- & -- & -- & -- & \\
\hline 182 & $10442835-5941238$ & 1.00 & $13.69 \pm * * * *$ & $12.99 \pm 0.03$ & $12.72 \pm 0.04$ & UAA & $0 \mathrm{cc}$ & $\mathrm{NaN}$ & $\mathrm{NaN}$ & \\
\hline 183 & $10442842-5948258$ & 0.33 & $14.23 \pm 0.08$ & $13.31 \pm 0.07$ & $13.02 \pm 0.06$ & AAA & $\mathrm{ccc}$ & 3.10 & 2.22 & \\
\hline 184 & $10442847-5941450$ & 0.68 & $13.96 \pm 0.03$ & $13.23 \pm 0.03$ & $12.96 \pm 0.04$ & AAA & $\mathrm{ccc}$ & 2.80 & 2.30 & \\
\hline 185 & -------- & -- & ----- & ----- & ----- & -- & -- & -- & -- & \\
\hline 186 & $10442850-5939200$ & 0.31 & $15.68 \pm 0.08$ & $14.72 \pm 0.07$ & $14.45 \pm 0.12$ & AAB & 000 & $\mathrm{NaN}$ & 1.22 & \\
\hline 187 & $10442865-5944140$ & 1.06 & $15.88 \pm 0.12$ & $14.85 \pm 0.08$ & $14.44 \pm 0.11$ & BAB & $\mathrm{ccc}$ & $\mathrm{NaN}$ & 1.10 & \\
\hline 188 & $10442857-5940527$ & 0.32 & $15.09 \pm 0.07$ & $14.64 \pm 0.07$ & $14.21 \pm 0.10$ & AAA & $\operatorname{css}$ & 4.81 & 1.66 & K-excess \\
\hline 189 & $10442906-5948165$ & 1.03 & $11.92 \pm 0.03$ & $11.40 \pm 0.03$ & $11.10 \pm 0.03$ & AAA & $\mathrm{ccc}$ & 4.08 & 7.25 & \\
\hline 190 & -------- & -- & ----- & ----- & ----- & -- & -- & -- & -- & \\
\hline 191 & $10442907-5941431$ & 1.56 & $12.64 \pm 0.04$ & $12.09 \pm 0.04$ & $11.92 \pm 0.04$ & AAA & $\mathrm{ccc}$ & 1.34 & 4.96 & \\
\hline 192 & $10442925-5943034$ & 0.25 & $15.33 \pm * * * *$ & $15.22 \pm 0.11$ & $14.13 \pm * * * *$ & UBU & $0 \mathrm{c} 0$ & $\mathrm{NaN}$ & $\mathrm{NaN}$ & \\
\hline 193 & -------- & -- & ----- & ----- & ----- & -- & -- & -- & -- & \\
\hline 194 & $10442933-5945005$ & 0.29 & $16.15 \pm 0.17$ & $15.10 \pm 0.10$ & $14.31 \pm * * * *$ & CAU & $\operatorname{cc} 0$ & $\mathrm{NaN}$ & 0.93 & \\
\hline 195 & $10442946-5936513$ & 0.15 & $15.09 \pm 0.05$ & $14.34 \pm 0.05$ & $14.00 \pm 0.07$ & AAA & 000 & 3.47 & 1.66 & \\
\hline 196 & $10442939-5937393$ & 1.52 & $14.68 \pm 0.05$ & $13.92 \pm 0.05$ & $13.67 \pm 0.06$ & AAA & $\mathrm{ccc}$ & 2.15 & 1.98 & \\
\hline
\end{tabular}


Table 2. continued.

\begin{tabular}{|c|c|c|c|c|c|c|c|c|c|c|}
\hline$N_{x}$ & 2 MASS J+ & Off." & $J$ mag & $H$ mag & $K_{\mathrm{s}} \mathrm{mag}$ & Ph.Q & Cont. & $A_{\mathrm{v}}$ & Mass & ID.flags \\
\hline 197 & $10442936-5946058$ & 0.30 & $15.54 \pm 0.08$ & $14.53 \pm 0.06$ & $14.20 \pm 0.08$ & $\overline{\mathrm{AAA}}$ & 000 & 2.88 & 1.31 & \\
\hline 198 & -------- & -- & ----- & ----- & ----- & -- & -- & -- & -- & \\
\hline 199 & $10442969-5944536$ & 0.65 & $15.26 \pm 0.08$ & $14.41 \pm 0.06$ & $14.08 \pm 0.09$ & AAA & $\operatorname{cc} 0$ & 3.26 & 1.50 & \\
\hline 200 & $10442976-5943327$ & 0.12 & $14.09 \pm 0.04$ & $13.34 \pm 0.03$ & $13.04 \pm 0.04$ & AAA & 000 & 3.28 & 2.26 & \\
\hline 201 & $10442981-5945339$ & 0.23 & $16.10 \pm 0.13$ & $15.14 \pm 0.11$ & $14.75 \pm 0.14$ & BBB & $\mathrm{ccc}$ & $\mathrm{NaN}$ & 0.96 & \\
\hline 202 & $10442998-5951037$ & 0.91 & $15.45 \pm 0.07$ & $14.34 \pm 0.06$ & $13.98 \pm 0.07$ & AAA & $\mathrm{ccc}$ & 3.84 & 1.37 & \\
\hline 203 & $10443000-5942182$ & 0.36 & $15.45 \pm 0.07$ & $14.33 \pm 0.05$ & $13.98 \pm 0.09$ & AAA & $\mathrm{c} 00$ & 3.66 & 1.37 & \\
\hline 204 & $10443001-5944477$ & 0.26 & $13.57 \pm * * * *$ & $13.24 \pm 0.04$ & $13.09 \pm 0.06$ & UAA & $0 \mathrm{cc}$ & $\mathrm{NaN}$ & $\mathrm{NaN}$ & \\
\hline 205 & $10443007-5941003$ & 0.36 & $15.03 \pm 0.07$ & $14.18 \pm 0.07$ & $13.80 \pm 0.09$ & AAA & 000 & 4.16 & 1.71 & \\
\hline 206 & $10443032-5940364$ & 0.18 & $15.27 \pm 0.07$ & $14.22 \pm 0.04$ & $14.07 \pm 0.08$ & AAA & 000 & $\mathrm{NaN}$ & 1.49 & \\
\hline 207 & $10443037-5937267$ & 0.29 & $8.84 \pm 0.02$ & $8.67 \pm 0.02$ & $8.58 \pm 0.03$ & AAA & $0 \mathrm{cc}$ & 2.27 & 18.65 & B0V \\
\hline 208 & $10443039-5944539$ & 0.28 & $15.65 \pm 0.09$ & $15.07 \pm 0.10$ & $13.99 \pm * * * *$ & AAU & $\operatorname{cc} 0$ & $\mathrm{NaN}$ & 1.23 & \\
\hline 209 & $10443054-5939246$ & 0.81 & $11.53 \pm 0.02$ & $11.01 \pm 0.02$ & $10.48 \pm 0.02$ & AAA & 000 & 9.32 & 8.69 & K-excess \\
\hline 210 & $10443060-5946290$ & 0.48 & $15.50 \pm 0.08$ & $14.58 \pm 0.07$ & $14.23 \pm 0.10$ & AAA & 000 & 3.11 & 1.33 & \\
\hline 211 & $10443061-5935361$ & 0.78 & $14.50 \pm 0.05$ & $13.63 \pm 0.05$ & $13.24 \pm 0.05$ & AAA & 000 & 4.67 & 2.09 & \\
\hline 212 & -------- & -- & ----- & ----- & ----- & -- & -- & -- & -- & \\
\hline 213 & $10443101-5942051$ & 1.17 & $14.83 \pm 0.05$ & $14.03 \pm 0.04$ & $13.85 \pm 0.06$ & AAA & 000 & 0.83 & 1.88 & \\
\hline 214 & $10443112-5942392$ & 0.23 & $16.42 \pm * * * *$ & $15.12 \pm 0.11$ & $14.84 \pm 0.16$ & UAB & $00 \mathrm{c}$ & $\mathrm{NaN}$ & $\mathrm{NaN}$ & \\
\hline 215 & $10443110-5943048$ & 0.07 & $14.85 \pm 0.04$ & $14.19 \pm * * * *$ & $13.94 \pm * * * *$ & AUU & pp0 & $\mathrm{NaN}$ & 1.86 & \\
\hline 216 & $10443106-5941277$ & 0.18 & $15.21 \pm 0.10$ & $14.89 \pm 0.11$ & $14.35 \pm 0.13$ & $\mathrm{ABB}$ & $\operatorname{cc} 0$ & $\mathrm{NaN}$ & 1.55 & \\
\hline 217 & -------- & -- & ----- & ----- & ----- & -- & -- & -- & -- & \\
\hline 218 & $10443120-5944186$ & 0.85 & $14.14 \pm * * * *$ & $15.59 \pm 0.20$ & $13.74 \pm * * * *$ & UCU & $0 \mathrm{c} 0$ & $\mathrm{NaN}$ & $\mathrm{NaN}$ & \\
\hline 219 & -------- & -- & ----- & ----- & ----- & -- & -- & -- & -- & \\
\hline 220 & -------- & -- & ----- & ----- & ----- & -- & -- & -- & -- & \\
\hline 221 & $10443181-5946123$ & 0.38 & $16.61 \pm * * * *$ & $15.30 \pm 0.12$ & $15.53 \pm * * * *$ & UBU & 000 & $\mathrm{NaN}$ & $\mathrm{NaN}$ & \\
\hline 222 & -------- & -- & ----- & ----- & ----- & -- & -- & -- & -- & \\
\hline 223 & $10443186-5938415$ & 0.14 & $14.57 \pm 0.05$ & $13.84 \pm 0.04$ & $13.58 \pm 0.05$ & AAA & 000 & 2.41 & 2.05 & \\
\hline 224 & $10443190-5944020$ & 0.56 & $14.47 \pm 0.08$ & $13.73 \pm 0.06$ & $13.09 \pm 0.05$ & AAA & $\operatorname{ccc}$ & 8.70 & 2.11 & K-excess \\
\hline 225 & $10443200-5943006$ & 0.61 & $15.62 \pm 0.10$ & $14.49 \pm 0.10$ & $14.23 \pm 0.10$ & AAA & $\mathrm{c} 0 \mathrm{c}$ & 1.52 & 1.25 & \\
\hline 226 & -------- & -- & ----- & ----- & ----- & -- & -- & -- & -- & \\
\hline 227 & -------- & -- & ----- & ----- & ----- & -- & -- & -- & -- & \\
\hline 228 & $10443233-5944309$ & 0.32 & $8.03 \pm 0.02$ & $7.99 \pm 0.04$ & $7.97 \pm 0.03$ & AAA & 000 & 1.06 & 21.67 & $\mathrm{O} 5 \mathrm{~V}((\mathrm{f}))$ \\
\hline 229 & $10443242-5942002$ & 0.32 & $16.41 \pm * * * *$ & $15.30 \pm 0.12$ & $15.50 \pm * * * *$ & UBU & 000 & $\mathrm{NaN}$ & $\mathrm{NaN}$ & \\
\hline 230 & $10443255-5944066$ & 0.05 & $11.97 \pm 0.03$ & $11.38 \pm 0.03$ & $10.69 \pm 0.02$ & AAA & $\mathrm{c} 00$ & $\mathrm{NaN}$ & 7.06 & K-excess \\
\hline 231 & $10443254-5943517$ & 0.17 & $14.00 \pm 0.04$ & $13.35 \pm 0.02$ & $13.20 \pm 0.03$ & AAE & $\mathrm{c} 00$ & $\mathrm{NaN}$ & 2.29 & \\
\hline 232 & -------- & -- & ----- & ----- & ----- & -- & -- & -- & -- & \\
\hline 233 & -------- & -- & ----- & ----- & ----- & -- & -- & -- & -- & \\
\hline 234 & $10443280-5943064$ & 0.34 & $13.54 \pm 0.03$ & $12.96 \pm 0.03$ & $12.73 \pm 0.04$ & AAA & 000 & 2.29 & 2.42 & \\
\hline 235 & $10443292-5947021$ & 0.40 & $15.32 \pm 0.07$ & $14.23 \pm 0.05$ & $13.91 \pm 0.07$ & AAA & 000 & 3.19 & 1.45 & \\
\hline 236 & $10443306-5941309$ & 0.43 & $15.18 \pm 0.06$ & $14.19 \pm 0.05$ & $14.10 \pm 0.08$ & AAA & 000 & $\mathrm{NaN}$ & 1.58 & \\
\hline 237 & $10443309-5940022$ & 0.48 & $14.48 \pm 0.04$ & $13.56 \pm 0.02$ & $13.35 \pm 0.04$ & EAE & 000 & $\mathrm{NaN}$ & 2.10 & \\
\hline 238 & $10443309-5945478$ & 0.45 & $15.08 \pm 0.08$ & $13.93 \pm * * * *$ & $13.41 \pm * * * *$ & AUU & 000 & $\mathrm{NaN}$ & 1.67 & \\
\hline 239 & $10443319-5949278$ & 0.77 & $16.29 \pm 0.14$ & $14.68 \pm 0.07$ & $13.90 \pm 0.06$ & BAA & $\mathrm{c} 00$ & $\mathrm{NaN}$ & 0.84 & \\
\hline 240 & $10443330-5935543$ & 0.19 & $14.99 \pm 0.08$ & $14.24 \pm 0.07$ & $13.87 \pm 0.08$ & AAA & $\operatorname{cc} 0$ & 4.03 & 1.75 & \\
\hline 241 & $10443376-5943129$ & 0.94 & $14.06 \pm * * * *$ & $13.70 \pm * * * *$ & $14.20 \pm 0.09$ & UUA & $00 \mathrm{c}$ & $\mathrm{NaN}$ & $\mathrm{NaN}$ & \\
\hline 242 & $10443374-5944154$ & 0.14 & $7.39 \pm 0.01$ & $7.39 \pm 0.03$ & $7.34 \pm 0.03$ & AAA & 000 & 1.49 & 24.03 & $\mathrm{O} 8 \mathrm{~V}$ \\
\hline 243 & -------- & -- & ----- & ----- & ----- & -- & -- & -- & -- & \\
\hline 244 & -------- & -- & ----- & ----- & ----- & -- & -- & -- & -- & \\
\hline 245 & $10443396-5937340$ & 0.22 & $14.22 \pm 0.04$ & $13.43 \pm 0.04$ & $13.13 \pm 0.04$ & AAA & $\mathrm{ccc}$ & 3.22 & 2.22 & \\
\hline 246 & $10443402-5948091$ & 0.26 & $15.84 \pm * * * *$ & $15.31 \pm 0.12$ & $14.12 \pm * * * *$ & UBU & 000 & $\mathrm{NaN}$ & $\mathrm{NaN}$ & \\
\hline 247 & $10443393-5942486$ & 0.20 & $14.56 \pm 0.06$ & $13.72 \pm 0.09$ & $13.32 \pm 0.09$ & AAA & $\mathrm{ccc}$ & 4.70 & 2.06 & \\
\hline 248 & $10443410-5947366$ & 0.62 & $16.09 \pm 0.12$ & $14.39 \pm 0.05$ & $13.56 \pm 0.05$ & BAA & $\mathrm{c} 00$ & $\mathrm{NaN}$ & 0.97 & \\
\hline 249 & $10443403-5943553$ & 1.08 & $12.86 \pm 0.03$ & $12.13 \pm 0.03$ & $11.68 \pm 0.03$ & AAA & ddd & 5.94 & 4.51 & \\
\hline 250 & -------- & -- & ----- & ----- & ----- & -- & -- & -- & -- & \\
\hline 251 & $10443492-5944059$ & 0.37 & $13.38 \pm * * * *$ & $12.69 \pm * * * *$ & $12.91 \pm 0.04$ & UUA & $00 \mathrm{c}$ & $\mathrm{NaN}$ & $\mathrm{NaN}$ & \\
\hline 252 & $10443499-5941423$ & 0.48 & $14.85 \pm 0.04$ & $14.07 \pm 0.05$ & $13.71 \pm 0.06$ & AAA & $\mathrm{ccc}$ & 3.95 & 1.87 & \\
\hline 253 & -------- & -- & ----- & ----- & ----- & -- & -- & -- & -- & \\
\hline 254 & $10443509-5945120$ & 0.17 & $15.61 \pm 0.08$ & $14.84 \pm 0.08$ & $14.41 \pm 0.10$ & AAA & 000 & 4.38 & 1.26 & \\
\hline 255 & -------- & -- & ----- & ----- & ----- & -- & -- & -- & -- & \\
\hline 256 & $10443538-5935346$ & 0.48 & $13.52 \pm 0.03$ & $12.77 \pm 0.03$ & $12.47 \pm 0.03$ & AAA & 000 & 3.37 & 2.42 & \\
\hline 257 & -------- & -- & ----- & ----- & ----- & -- & -- & -- & -- & \\
\hline 258 & $10443547-5948082$ & 1.05 & $12.37 \pm 0.02$ & $12.26 \pm 0.02$ & $12.30 \pm 0.03$ & AAA & 000 & $\mathrm{NaN}$ & 5.69 & \\
\hline 259 & $10443544-5940461$ & 0.39 & $16.51 \pm 0.21$ & $15.11 \pm 0.11$ & $15.02 \pm 0.19$ & $\mathrm{CBC}$ & $\mathrm{c} 0 \mathrm{c}$ & $\mathrm{NaN}$ & 0.69 & \\
\hline 260 & $10443552-5943509$ & 0.63 & $13.98 \pm 0.03$ & $13.27 \pm 0.03$ & $12.99 \pm 0.04$ & AAA & 000 & 2.97 & 2.29 & \\
\hline
\end{tabular}


J. F. Albacete-Colombo et al.: An X-ray survey of low-mass stars in Trumpler 16 with Chandra, Online Material p 28

Table 2. continued.

\begin{tabular}{|c|c|c|c|c|c|c|c|c|c|c|}
\hline$N_{x}$ & 2MASS J+ & Off." & $J$ mag & $H$ mag & $K_{\mathrm{s}} \mathrm{mag}$ & Ph.Q & Cont. & $A_{\mathrm{v}}$ & Mass & ID.flags \\
\hline 261 & -------- & -- & ----- & ----- & ----- & -- & -- & $\overline{--}$ & -- & \\
\hline 262 & -------- & -- & ----- & ----- & ----- & -- & -- & -- & -- & \\
\hline 263 & -------- & -- & ----- & ----- & ----- & -- & -- & -- & -- & \\
\hline 264 & $10443571-5945028$ & 0.32 & $15.51 \pm 0.09$ & $15.16 \pm 0.11$ & $14.60 \pm 0.13$ & $\mathrm{ABB}$ & $\mathrm{cp} 0$ & $\mathrm{NaN}$ & 1.33 & \\
\hline 265 & -------- & -- & ----- & ----- & ----- & -- & -- & -- & -- & \\
\hline 266 & $10443586-5944469$ & 0.23 & $14.44 \pm 0.04$ & $13.69 \pm 0.03$ & $13.59 \pm 0.05$ & AAA & 000 & $\mathrm{NaN}$ & 2.12 & \\
\hline 267 & $10443601-5944570$ & 0.33 & $15.47 \pm 0.08$ & $14.59 \pm 0.09$ & $14.36 \pm 0.10$ & AAA & $\mathrm{ccc}$ & 0.51 & 1.36 & \\
\hline 268 & $10443606-5942585$ & 0.48 & $16.03 \pm 0.12$ & $15.19 \pm 0.13$ & $14.40 \pm * * * *$ & BBU & $\operatorname{cc} 0$ & $\mathrm{NaN}$ & 1.01 & \\
\hline 269 & -------- & -- & ----- & ----- & ---- & -- & -- & -- & -- & \\
\hline 270 & $10443618-5951085$ & 0.30 & $13.39 \pm 0.05$ & $13.26 \pm 0.04$ & $13.29 \pm 0.06$ & AAA & $\mathrm{ccc}$ & $\mathrm{NaN}$ & 2.46 & \\
\hline 271 & $10443633-5944111$ & 1.19 & $14.17 \pm 0.05$ & $13.48 \pm 0.05$ & $13.31 \pm 0.06$ & AAA & $\mathrm{ccc}$ & 0.88 & 2.24 & \\
\hline 272 & $10443634-5939443$ & 0.28 & $14.16 \pm 0.08$ & $13.59 \pm 0.08$ & $12.94 \pm 0.11$ & AAA & $\operatorname{ccc}$ & 8.96 & 2.24 & K-excess \\
\hline 273 & $10443637-5950429$ & 0.28 & $14.25 \pm 0.04$ & $13.14 \pm 0.03$ & $12.59 \pm 0.04$ & AAA & 000 & 7.37 & 2.22 & \\
\hline 274 & $10443648-5939032$ & 0.57 & $14.87 \pm 0.12$ & $13.77 \pm * * * *$ & $13.09 \pm * * * *$ & BUU & $\mathrm{c} 00$ & $\mathrm{NaN}$ & 1.85 & \\
\hline 275 & $10443662-5943051$ & 1.89 & $14.93 \pm 0.05$ & $14.09 \pm 0.04$ & $13.83 \pm * * * *$ & AAU & $\operatorname{cc} 0$ & $\mathrm{NaN}$ & 1.81 & \\
\hline 276 & $10443668-5944239$ & 0.36 & $14.83 \pm 0.05$ & $13.84 \pm 0.04$ & $13.47 \pm 0.05$ & AAA & $\operatorname{cc} 0$ & 4.23 & 1.88 & \\
\hline 277 & ------- & -- & ----- & ----- & ----- & -- & -- & -- & -- & \\
\hline 278 & $10443666-5946218$ & 0.06 & $14.14 \pm 0.03$ & $13.21 \pm 0.04$ & $12.63 \pm 0.03$ & AAA & 000 & 7.78 & 2.25 & K-excess \\
\hline 279 & $10443668-5945563$ & 0.42 & $15.77 \pm 0.09$ & $15.04 \pm 0.09$ & $14.78 \pm 0.14$ & $\mathrm{AAB}$ & 000 & $\mathrm{NaN}$ & 1.16 & \\
\hline 280 & ------- & -- & ----- & ----- & ----- & -- & -- & -- & -- & \\
\hline 281 & $10443668-5947296$ & 0.24 & $9.38 \pm 0.02$ & $9.14 \pm 0.02$ & $9.01 \pm 0.02$ & AAA & $\mathrm{s} 00$ & 2.94 & 16.64 & $\mathrm{O} 8 \mathrm{~V}$ \\
\hline 282 & $10443676-5950098$ & 0.53 & $15.12 \pm 0.08$ & $13.97 \pm 0.06$ & $13.49 \pm 0.06$ & AAA & $\operatorname{ccc}$ & 5.92 & 1.63 & \\
\hline 283 & $10443679-5944063$ & 1.63 & $15.65 \pm 0.09$ & $14.93 \pm 0.09$ & $15.02 \pm * * * *$ & AAU & $\operatorname{cc} 0$ & $\mathrm{NaN}$ & 1.24 & \\
\hline 284 & $10443699-5939441$ & 0.49 & $14.40 \pm 0.11$ & $13.64 \pm 0.10$ & $12.22 \pm * * * *$ & BAU & $\operatorname{cc} 0$ & $\mathrm{NaN}$ & 2.15 & \\
\hline 285 & $10443712-5937356$ & 0.49 & $14.58 \pm * * * *$ & $14.31 \pm 0.11$ & $13.51 \pm * * * *$ & UBU & $0 \mathrm{c} 0$ & $\mathrm{NaN}$ & $\mathrm{NaN}$ & \\
\hline 286 & $10443718-5940014$ & 0.80 & $10.52 \pm 0.02$ & $10.37 \pm 0.02$ & $10.27 \pm 0.02$ & AAA & 000 & 2.32 & 12.44 & B $0.5 \mathrm{~V}$ \\
\hline 287 & -------- & -- & ----- & ----- & ----- & -- & -- & -- & -- & \\
\hline 288 & -------- & -- & ----- & ----- & ----- & -- & -- & -- & -- & \\
\hline 289 & $10443734-5943366$ & 0.42 & $14.52 \pm 0.05$ & $14.08 \pm 0.06$ & $13.77 \pm 0.06$ & AAA & 000 & 3.06 & 2.08 & \\
\hline 290 & $10443748-5944474$ & 0.29 & $15.80 \pm 0.11$ & $14.83 \pm 0.08$ & $14.61 \pm 0.13$ & $\mathrm{AAB}$ & $\mathrm{c} 00$ & $\mathrm{NaN}$ & 1.14 & \\
\hline 291 & $10443756-5942459$ & 0.42 & $15.90 \pm 0.11$ & $14.87 \pm 0.08$ & $14.62 \pm 0.14$ & BAB & $00 \mathrm{c}$ & $\mathrm{NaN}$ & 1.09 & \\
\hline 292 & -------- & -- & ----- & ----- & ----- & -- & -- & -- & -- & \\
\hline 293 & $10443766-5944309$ & 0.30 & $15.00 \pm * * * *$ & $15.09 \pm 0.12$ & $13.47 \pm * * * *$ & UBU & $0 \mathrm{c} 0$ & $\mathrm{NaN}$ & $\mathrm{NaN}$ & \\
\hline 294 & -------- & -- & ----- & ----- & ----- & -- & -- & -- & -- & \\
\hline 295 & -------- & -- & ----- & ----- & ----- & -- & -- & -- & -- & \\
\hline 296 & $10443772-5942167$ & 0.14 & $15.02 \pm 0.05$ & $14.13 \pm 0.05$ & $13.74 \pm 0.06$ & AAA & 000 & 4.40 & 1.72 & \\
\hline 297 & $10443782-5943310$ & 0.26 & $14.93 \pm 0.06$ & $14.33 \pm 0.06$ & $13.83 \pm 0.07$ & AAA & $\operatorname{ccc}$ & 6.20 & 1.80 & K-excess \\
\hline 298 & -------- & -- & ----- & ----- & ----- & -- & -- & -- & -- & \\
\hline 299 & $10443789-5935350$ & 0.24 & $13.48 \pm 0.03$ & $12.80 \pm 0.03$ & $12.61 \pm 0.03$ & AAA & 000 & 1.61 & 2.44 & \\
\hline 300 & -------- & -- & ----- & ----- & ----- & -- & -- & -- & -- & \\
\hline 301 & $10443807-5944350$ & 1.83 & $13.39 \pm 0.03$ & $12.52 \pm 0.02$ & $11.99 \pm 0.03$ & AAA & 000 & 7.12 & 2.46 & K-excess \\
\hline 302 & $10443808-5943260$ & 0.32 & $12.83 \pm 0.04$ & $12.23 \pm 0.05$ & $12.01 \pm 0.05$ & AAA & $\mathrm{ccc}$ & 2.19 & 4.58 & \\
\hline 303 & $10443803-5940267$ & 0.34 & $15.10 \pm 0.06$ & $14.01 \pm 0.05$ & $13.56 \pm 0.06$ & AAA & $\mathrm{ccc}$ & 5.56 & 1.65 & \\
\hline 304 & $10443808-5941313$ & 0.89 & $13.72 \pm 0.04$ & $12.94 \pm 0.05$ & $12.65 \pm 0.04$ & AAA & 000 & 3.18 & 2.37 & \\
\hline 305 & $10443814-5944263$ & 0.39 & $15.46 \pm 0.08$ & $14.79 \pm 0.09$ & $14.29 \pm 0.10$ & AAA & $\mathrm{c} 00$ & 5.95 & 1.36 & K-excess \\
\hline 306 & $10443830-5939135$ & 0.34 & $15.65 \pm * * * *$ & $14.68 \pm 0.09$ & $14.58 \pm * * * *$ & UAU & $0 \mathrm{c} 0$ & $\mathrm{NaN}$ & $\mathrm{NaN}$ & \\
\hline 307 & $10443816-5943114$ & 1.17 & $13.22 \pm 0.03$ & $12.46 \pm 0.03$ & $12.17 \pm 0.03$ & AAA & $\operatorname{ccc}$ & 3.27 & 3.55 & Mass-deg. \\
\hline 308 & $10443822-5943340$ & 0.35 & $14.82 \pm 0.07$ & $13.80 \pm 0.05$ & $13.67 \pm 0.07$ & AAA & $\mathrm{ccc}$ & 0.16 & 1.89 & \\
\hline 309 & $10443837-5943444$ & 0.46 & $15.22 \pm 0.09$ & $14.13 \pm * * * *$ & $13.69 \pm * * * *$ & AUU & $\mathrm{c} 00$ & $\mathrm{NaN}$ & 1.54 & \\
\hline 310 & -------- & -- & ----- & ----- & ----- & -- & -- & -- & -- & \\
\hline 311 & -------- & -- & ----- & ----- & ----- & -- & -- & -- & -- & \\
\hline 312 & -------- & -- & ----- & ----- & ----- & -- & -- & -- & -- & \\
\hline 313 & $10443862-5947394$ & 0.37 & $16.02 \pm 0.12$ & $15.03 \pm 0.10$ & $14.68 \pm 0.14$ & BAB & $\operatorname{ccc}$ & $\mathrm{NaN}$ & 1.01 & \\
\hline 314 & $10443868-5944429$ & 0.11 & $15.10 \pm 0.05$ & $14.24 \pm 0.05$ & $14.02 \pm 0.08$ & AAA & 000 & 0.95 & 1.65 & \\
\hline 315 & $10443853-5948487$ & 0.26 & $15.71 \pm 0.08$ & $14.35 \pm 0.06$ & $13.88 \pm 0.06$ & AAA & 000 & 5.68 & 1.20 & \\
\hline 316 & -------- & -- & ----- & ----- & ----- & -- & -- & -- & -- & \\
\hline 317 & $10443902-5946535$ & 1.78 & $14.83 \pm 0.07$ & $13.96 \pm 0.06$ & $13.69 \pm 0.09$ & AAA & $\mathrm{ccc}$ & 2.46 & 1.88 & \\
\hline 318 & $10443927-5942584$ & 0.75 & $15.95 \pm * * * *$ & $14.95 \pm * * * *$ & $14.80 \pm 0.15$ & UUB & $00 \mathrm{c}$ & $\mathrm{NaN}$ & $\mathrm{NaN}$ & \\
\hline
\end{tabular}


Table 2. continued.

\begin{tabular}{|c|c|c|c|c|c|c|c|c|c|c|}
\hline$\overline{N_{x}}$ & 2MASS J+ & Off." & $J \mathrm{mag}$ & $H \mathrm{mag}$ & $K_{\mathrm{s}} \mathrm{mag}$ & Ph.Q & Cont. & $A_{\mathrm{v}}$ & Mass & ID.flags \\
\hline 319 & $10443941-5940402$ & 0.68 & $16.13 \pm * * * *$ & $15.71 \pm 0.19$ & $15.08 \pm * * * *$ & UCU & $0 \mathrm{c} 0$ & $\mathrm{NaN}$ & $\mathrm{NaN}$ & \\
\hline 320 & $10443942-5946395$ & 0.50 & $14.95 \pm 0.05$ & $13.98 \pm 0.04$ & $13.39 \pm 0.04$ & AEE & $\mathrm{c} 0 \mathrm{c}$ & $\mathrm{NaN}$ & 1.78 & \\
\hline 321 & $10443941-5940402$ & 0.49 & $16.13 \pm * * * *$ & $15.71 \pm 0.19$ & $15.08 \pm * * * *$ & UCU & $0 \mathrm{c} 0$ & $\mathrm{NaN}$ & $\mathrm{NaN}$ & \\
\hline 322 & $10443972-5948362$ & 0.74 & $15.27 \pm 0.06$ & $13.83 \pm 0.04$ & $13.43 \pm 0.05$ & AAA & $\operatorname{ccc}$ & 4.73 & 1.49 & \\
\hline 323 & $10443991-5949243$ & 0.32 & $16.83 \pm * * * *$ & $14.81 \pm 0.08$ & $14.70 \pm 0.14$ & UAB & 000 & $\mathrm{NaN}$ & $\mathrm{NaN}$ & \\
\hline 324 & -------- & -- & ----- & ----- & ----- & -- & -- & -- & -- & \\
\hline 325 & $10443989-5943582$ & 0.07 & $13.39 \pm * * * *$ & $12.78 \pm * * * *$ & $12.46 \pm 0.04$ & UUA & $00 \mathrm{c}$ & $\mathrm{NaN}$ & $\mathrm{NaN}$ & \\
\hline 326 & $10444012-5942218$ & 0.36 & $15.39 \pm * * * *$ & $14.59 \pm * * * *$ & $14.66 \pm 0.13$ & UUB & $00 \mathrm{c}$ & $\mathrm{NaN}$ & $\mathrm{NaN}$ & \\
\hline 327 & $10444009-5945569$ & 0.62 & $15.14 \pm 0.07$ & $14.28 \pm 0.07$ & $13.98 \pm 0.08$ & AAA & 000 & 2.81 & 1.61 & \\
\hline 328 & -------- & -- & ----- & ----- & ----- & -- & -- & -- & -- & \\
\hline 329 & $10444001-5945479$ & 0.17 & $15.22 \pm 0.06$ & $14.20 \pm 0.06$ & $13.80 \pm 0.06$ & AAA & 000 & 4.56 & 1.54 & \\
\hline 330 & $10444008-5941179$ & 0.78 & $14.77 \pm 0.04$ & $13.88 \pm 0.03$ & $13.72 \pm 0.05$ & AEA & $00 \mathrm{c}$ & $\mathrm{NaN}$ & 1.92 & \\
\hline 331 & $10444014-5946117$ & 0.35 & $15.88 \pm 0.09$ & $14.90 \pm 0.09$ & $14.59 \pm 0.12$ & AAB & 000 & $\mathrm{NaN}$ & 1.10 & \\
\hline 332 & $10444058-5934579$ & 0.49 & $14.87 \pm 0.06$ & $14.06 \pm 0.06$ & $13.72 \pm 0.08$ & AAA & 000 & 3.60 & 1.85 & \\
\hline 333 & $10444076-5944454$ & 2.08 & $16.10 \pm 0.14$ & $14.98 \pm 0.11$ & $14.26 \pm * * * *$ & BBU & $\operatorname{cc} 0$ & $\mathrm{NaN}$ & 0.96 & \\
\hline 334 & $10444086-5945247$ & 0.18 & $13.26 \pm 0.03$ & $12.97 \pm 0.03$ & $12.87 \pm 0.04$ & AAA & 000 & $\mathrm{NaN}$ & 2.50 & \\
\hline 335 & $10444093-5944011$ & 0.33 & $15.63 \pm * * * *$ & $15.31 \pm 0.12$ & $14.57 \pm * * * *$ & UBU & $0 \mathrm{c} 0$ & $\mathrm{NaN}$ & $\mathrm{NaN}$ & \\
\hline 336 & $10444111-5940466$ & 0.48 & $15.24 \pm 0.09$ & $14.66 \pm 0.09$ & $12.91 \pm * * * *$ & AAU & $\operatorname{cc0}$ & $\mathrm{NaN}$ & 1.52 & \\
\hline 337 & -------- & -- & ----- & ----- & ----- & -- & -- & -- & -- & \\
\hline 338 & $10444109-5945597$ & 0.09 & $16.10 \pm 0.14$ & $15.27 \pm 0.14$ & $16.13 \pm * * * *$ & BBU & 000 & $\mathrm{NaN}$ & 0.96 & \\
\hline 339 & $10444108-5944228$ & 0.67 & $14.89 \pm 0.05$ & $14.00 \pm 0.05$ & $13.61 \pm 0.06$ & AAA & 000 & 4.61 & 1.83 & \\
\hline 340 & -------- & -- & ----- & ----- & ----- & -- & -- & -- & -- & \\
\hline 341 & -------- & -- & ----- & ----- & ----- & -- & -- & -- & -- & \\
\hline 342 & $10444114-5938194$ & 0.41 & $13.25 \pm * * * *$ & $12.61 \pm 0.04$ & $12.29 \pm 0.04$ & UAA & $0 \mathrm{cc}$ & $\mathrm{NaN}$ & $\mathrm{NaN}$ & \\
\hline 343 & -------- & -- & ----- & ----- & ----- & -- & -- & -- & -- & \\
\hline 344 & $10444128-5943031$ & 0.56 & $15.89 \pm 0.12$ & $14.71 \pm 0.07$ & $14.29 \pm 0.09$ & BAA & $\operatorname{ccc}$ & $\mathrm{NaN}$ & 1.09 & \\
\hline 345 & $10444130-5944087$ & 0.59 & $13.91 \pm 0.04$ & $13.20 \pm 0.04$ & $12.98 \pm 0.04$ & AAA & $\operatorname{cc} 0$ & 1.88 & 2.31 & \\
\hline 346 & -------- & -- & ----- & ----- & ----- & -- & -- & -- & -- & \\
\hline 347 & $10444150-5950319$ & 0.37 & $13.96 \pm 0.03$ & $12.58 \pm 0.02$ & $11.84 \pm 0.03$ & AAA & 000 & 11.08 & 2.30 & \\
\hline 348 & $10444155-5942091$ & 0.28 & $15.90 \pm * * * *$ & $15.09 \pm 0.10$ & $15.83 \pm * * * *$ & UAU & 000 & $\mathrm{NaN}$ & $\mathrm{NaN}$ & \\
\hline 349 & $10444160-5947046$ & 0.29 & $13.53 \pm 0.11$ & $12.89 \pm 0.09$ & $12.79 \pm 0.09$ & BAA & $\mathrm{ccc}$ & $\mathrm{NaN}$ & 2.42 & \\
\hline 350 & $10444170-5942543$ & 0.45 & $12.73 \pm * * * *$ & $12.41 \pm 0.04$ & $12.34 \pm 0.04$ & UAA & $0 \mathrm{cc}$ & $\mathrm{NaN}$ & $\mathrm{NaN}$ & \\
\hline 351 & $10444176-5940478$ & 0.32 & $13.01 \pm 0.04$ & $12.41 \pm 0.04$ & $12.08 \pm * * * *$ & AAU & $\operatorname{cc} 0$ & $\mathrm{NaN}$ & 3.55 & Mass-deg. \\
\hline 352 & $10444176-5946563$ & 0.25 & $7.80 \pm 0.01$ & $7.74 \pm 0.04$ & $7.64 \pm 0.03$ & AAA & 000 & 2.32 & 22.51 & $\mathrm{O} 6 \mathrm{~V}((\mathrm{f}))$ \\
\hline 353 & $10444196-5940227$ & 0.53 & $16.55 \pm * * * *$ & $16.13 \pm * * * *$ & $14.88 \pm 0.17$ & UUC & $00 \mathrm{c}$ & $\mathrm{NaN}$ & $\mathrm{NaN}$ & \\
\hline 354 & $10444197-5942160$ & 0.63 & $14.03 \pm 0.05$ & $13.41 \pm 0.04$ & $13.21 \pm 0.05$ & AAA & $\mathrm{ccc}$ & 1.64 & 2.28 & \\
\hline 355 & -------- & -- & ----- & ----- & ----- & -- & -- & -- & -- & \\
\hline 356 & $10444217-5942582$ & 0.31 & $15.55 \pm 0.09$ & $14.61 \pm 0.07$ & $14.44 \pm 0.11$ & AAB & $\mathrm{ccc}$ & $\mathrm{NaN}$ & 1.31 & \\
\hline 357 & $10444215-5942431$ & 0.21 & $14.83 \pm 0.07$ & $14.06 \pm 0.06$ & $13.87 \pm 0.08$ & AAA & $\mathrm{ccc}$ & 0.84 & 1.88 & \\
\hline 358 & -------- & -- & ----- & ----- & ----- & -- & -- & -- & -- & \\
\hline 359 & $10444236-5944043$ & 0.30 & $15.25 \pm 0.06$ & $14.25 \pm 0.06$ & $14.06 \pm 0.08$ & AAA & $\mathrm{ccc}$ & 0.58 & 1.51 & \\
\hline 360 & $10444237-5942524$ & 0.13 & $14.25 \pm * * * *$ & $14.90 \pm 0.08$ & $14.35 \pm 0.10$ & UAA & $0 \mathrm{cc}$ & $\mathrm{NaN}$ & $\mathrm{NaN}$ & \\
\hline 361 & $10444250-5942150$ & 0.38 & $15.50 \pm 0.09$ & $14.79 \pm 0.08$ & $14.40 \pm 0.10$ & AAA & $\mathrm{ccc}$ & 1.59 & 1.34 & \\
\hline 362 & $10444264-5941235$ & 0.66 & $13.93 \pm 0.03$ & $12.83 \pm 0.03$ & $12.11 \pm 0.03$ & AAA & $\mathrm{ccc}$ & 10.26 & 2.31 & K-excess \\
\hline 363 & -------- & -- & ----- & ----- & ----- & -- & -- & -- & -- & \\
\hline 364 & $10444285-5943539$ & 0.45 & $15.21 \pm 0.06$ & $14.50 \pm 0.06$ & $14.25 \pm 0.10$ & AAA & 000 & 0.02 & 1.54 & \\
\hline 365 & $10444284-5946453$ & 0.25 & $14.82 \pm * * * *$ & $14.92 \pm 0.12$ & $14.48 \pm 0.11$ & UBA & $0 \mathrm{cc}$ & $\mathrm{NaN}$ & $\mathrm{NaN}$ & \\
\hline 366 & $10444297-5940173$ & 0.27 & $15.40 \pm 0.08$ & $14.34 \pm 0.06$ & $14.02 \pm 0.07$ & AAA & $\mathrm{c} 0 \mathrm{c}$ & 3.07 & 1.40 & \\
\hline 367 & $10444308-5940245$ & 0.45 & $15.55 \pm 0.11$ & $14.11 \pm * * * *$ & $13.89 \pm * * * *$ & AUU & $\mathrm{c} 00$ & $\mathrm{NaN}$ & 1.31 & \\
\hline 368 & $10444309-5945059$ & 0.51 & $16.69 \pm * * * *$ & $15.20 \pm 0.09$ & $14.60 \pm * * * *$ & UAU & 000 & $\mathrm{NaN}$ & $\mathrm{NaN}$ & \\
\hline 369 & $10444316-5942178$ & 0.46 & $15.90 \pm 0.11$ & $15.14 \pm 0.11$ & $14.52 \pm * * * *$ & $\mathrm{ABU}$ & $\operatorname{cc} 0$ & $\mathrm{NaN}$ & 1.09 & \\
\hline 370 & $10444321-5945532$ & 0.72 & $15.91 \pm 0.14$ & $14.87 \pm 0.08$ & $14.36 \pm 0.10$ & BAA & $\mathrm{ccc}$ & $\mathrm{NaN}$ & 1.08 & \\
\hline 371 & $10444323-5943466$ & 0.36 & $15.71 \pm * * * *$ & $15.57 \pm 0.15$ & $14.94 \pm 0.16$ & UBC & $0 \mathrm{cc}$ & $\mathrm{NaN}$ & $\mathrm{NaN}$ & \\
\hline 372 & $10444329-5951202$ & 0.34 & $14.86 \pm * * * *$ & $13.60 \pm * * * *$ & $13.14 \pm 0.06$ & UUA & $00 \mathrm{c}$ & $\mathrm{NaN}$ & $\mathrm{NaN}$ & \\
\hline 373 & $10444334-5947295$ & 0.66 & $16.19 \pm 0.14$ & $14.92 \pm 0.07$ & $14.51 \pm 0.11$ & BAA & $\mathrm{ccc}$ & $\mathrm{NaN}$ & 0.91 & \\
\hline 374 & $10444332-5944513$ & 0.51 & $15.82 \pm 0.10$ & $15.01 \pm 0.10$ & $14.15 \pm * * * *$ & AAU & $\operatorname{cc} 0$ & $\mathrm{NaN}$ & 1.13 & \\
\hline 375 & $10444351-5941325$ & 0.77 & $15.75 \pm 0.09$ & $14.62 \pm 0.07$ & $14.26 \pm 0.09$ & AAA & $\operatorname{ccc}$ & 3.56 & 1.17 & \\
\hline 376 & $10444353-5947178$ & 0.27 & $13.73 \pm 0.02$ & $12.97 \pm 0.03$ & $12.69 \pm 0.02$ & AAA & 000 & 3.09 & 2.36 & \\
\hline 377 & $10444357-5945383$ & 0.49 & $15.48 \pm 0.09$ & $14.58 \pm 0.07$ & $14.26 \pm 0.12$ & $\mathrm{AAB}$ & $\mathrm{ccc}$ & $\mathrm{NaN}$ & 1.35 & \\
\hline 378 & $10444387-5941145$ & 0.47 & $15.46 \pm 0.08$ & $14.28 \pm 0.05$ & $14.02 \pm 0.08$ & AAA & $\mathrm{c} 0 \mathrm{c}$ & 1.80 & 1.36 & \\
\hline 379 & $10444395-5939557$ & 0.15 & $14.55 \pm 0.05$ & $13.75 \pm 0.05$ & $13.52 \pm 0.04$ & AEA & $\mathrm{ccc}$ & $\mathrm{NaN}$ & 2.06 & \\
\hline 380 & -------- & -- & ----- & ----- & ----- & -- & -- & -- & -- & \\
\hline 381 & $10444405-5939597$ & 0.02 & $15.93 \pm 0.12$ & $14.58 \pm 0.07$ & $15.02 \pm 0.19$ & $\mathrm{BAC}$ & $\mathrm{ccc}$ & $\mathrm{NaN}$ & 1.07 & \\
\hline 382 & $10444430-5951253$ & 0.25 & $13.50 \pm 0.07$ & $12.27 \pm * * * *$ & $12.44 \pm 0.09$ & AUA & $\mathrm{c} 0 \mathrm{c}$ & $\mathrm{NaN}$ & 2.43 & \\
\hline 383 & $10444430-5942333$ & 1.58 & $11.73 \pm 0.03$ & $11.51 \pm 0.04$ & $11.42 \pm 0.05$ & AAA & $00 \mathrm{c}$ & 0.20 & 7.95 & \\
\hline 384 & $10444422-5945375$ & 1.80 & $14.84 \pm 0.06$ & $13.99 \pm 0.05$ & $13.37 \pm 0.06$ & AAA & $\mathrm{ccc}$ & 8.50 & 1.88 & K-excess \\
\hline
\end{tabular}


J. F. Albacete-Colombo et al.: An X-ray survey of low-mass stars in Trumpler 16 with Chandra, Online Material p 30

Table 2. continued.

\begin{tabular}{|c|c|c|c|c|c|c|c|c|c|c|}
\hline$\overline{N_{x}}$ & 2MASS J+ & Off." & $J \mathrm{mag}$ & $H$ mag & $K_{\mathrm{s}} \mathrm{mag}$ & Ph.Q & Cont. & $A_{\mathrm{v}}$ & Mass & ID.flags \\
\hline 385 & $10444425-5948388$ & 0.38 & $17.23 \pm * * * *$ & $15.52 \pm 0.15$ & $16.53 \pm * * * *$ & $\overline{\mathrm{UBU}}$ & 000 & $\mathrm{NaN}$ & $\mathrm{NaN}$ & \\
\hline 386 & -------- & -- & ----- & ----- & ----- & -- & -- & -- & -- & \\
\hline 387 & $10444433-5938318$ & 0.30 & $14.35 \pm 0.04$ & $13.25 \pm 0.02$ & $12.84 \pm 0.04$ & AAA & 000 & 5.12 & 2.18 & \\
\hline 388 & $10444433-5945595$ & 0.25 & $13.21 \pm 0.03$ & $12.71 \pm 0.04$ & $12.57 \pm 0.04$ & AAA & 000 & 0.84 & 3.55 & Mass-deg. \\
\hline 389 & $10444449-5945283$ & 0.05 & $16.01 \pm * * * *$ & $15.30 \pm 0.12$ & $14.53 \pm * * * *$ & UBU & 000 & $\mathrm{NaN}$ & $\mathrm{NaN}$ & \\
\hline 390 & $10444461-5944135$ & 0.41 & $12.19 \pm 0.02$ & $12.09 \pm 0.02$ & $11.98 \pm 0.02$ & AAA & 000 & 0.38 & 6.26 & \\
\hline 391 & $10444485-5945593$ & 0.46 & $13.81 \pm * * * *$ & $14.52 \pm 0.06$ & $14.41 \pm 0.10$ & UAA & $0 \mathrm{cc}$ & $\mathrm{NaN}$ & $\mathrm{NaN}$ & \\
\hline 392 & $10444528-5939192$ & 0.06 & $12.64 \pm * * * *$ & $12.08 \pm 0.03$ & $11.80 \pm 0.04$ & UAA & $0 \mathrm{cc}$ & $\mathrm{NaN}$ & $\mathrm{NaN}$ & \\
\hline 393 & $10444534-5936104$ & 0.40 & $15.32 \pm 0.08$ & $14.51 \pm 0.06$ & $14.07 \pm 0.07$ & AAA & 000 & 5.19 & 1.45 & \\
\hline 394 & $10444549-5940348$ & 0.47 & $14.23 \pm * * * *$ & $14.92 \pm 0.15$ & $14.30 \pm 0.14$ & UBB & $0 \mathrm{cc}$ & $\mathrm{NaN}$ & $\mathrm{NaN}$ & \\
\hline 395 & -------- & -- & ----- & ----- & ----- & -- & -- & -- & -- & \\
\hline 396 & $10444591-5945210$ & 1.27 & $15.78 \pm 0.09$ & $15.09 \pm 0.09$ & $14.51 \pm 0.11$ & AAA & sss & 7.25 & 1.16 & K-excess \\
\hline 397 & $10444601-5946595$ & 0.61 & $15.40 \pm 0.08$ & $14.40 \pm 0.06$ & $13.88 \pm 0.06$ & AAA & $\mathrm{c} 0 \mathrm{~s}$ & 6.60 & 1.40 & \\
\hline 398 & -------- & -- & ----- & ----- & ----- & -- & -- & -- & -- & \\
\hline 399 & $10444616-5942293$ & 0.24 & $15.29 \pm 0.08$ & $14.23 \pm 0.06$ & $13.83 \pm 0.07$ & AAA & $\mathrm{c} 0 \mathrm{c}$ & 4.51 & 1.48 & \\
\hline 400 & $10444621-5944493$ & 0.18 & $14.49 \pm 0.05$ & $13.71 \pm 0.06$ & $13.48 \pm 0.08$ & AAA & $\mathrm{ccc}$ & 1.89 & 2.09 & \\
\hline 401 & $10444654-5936055$ & 0.33 & $14.74 \pm * * * *$ & $13.82 \pm * * * *$ & $13.64 \pm 0.06$ & UUA & 000 & $\mathrm{NaN}$ & $\mathrm{NaN}$ & \\
\hline 402 & $10444646-5941306$ & 0.27 & $16.06 \pm 0.13$ & $14.80 \pm 0.08$ & $14.40 \pm 0.10$ & BAA & $\mathrm{ccc}$ & $\mathrm{NaN}$ & 0.99 & \\
\hline 403 & $10444673-5939009$ & 0.67 & $14.67 \pm * * * *$ & $15.06 \pm 0.11$ & $13.38 \pm * * * *$ & UBU & $0 \mathrm{c} 0$ & $\mathrm{NaN}$ & $\mathrm{NaN}$ & \\
\hline 404 & $10444699-5943266$ & 2.10 & $15.25 \pm 0.08$ & $14.39 \pm 0.06$ & $14.03 \pm 0.09$ & AAA & $\mathrm{ccc}$ & 3.68 & 1.51 & \\
\hline 405 & $10444708-5938127$ & 0.13 & $15.64 \pm 0.11$ & $14.79 \pm 0.10$ & $14.23 \pm 0.09$ & BAA & s0c & $\mathrm{NaN}$ & 1.25 & \\
\hline 406 & $10444732-5941170$ & 0.51 & $12.22 \pm * * * *$ & $11.80 \pm * * * *$ & $11.66 \pm 0.04$ & UUA & $00 \mathrm{c}$ & $\mathrm{NaN}$ & $\mathrm{NaN}$ & \\
\hline 407 & $10444729-5943532$ & 0.21 & $8.34 \pm 0.01$ & $8.34 \pm 0.02$ & $8.29 \pm 0.02$ & AAA & 000 & 1.71 & 20.49 & B $0.2 \mathrm{~V}$ \\
\hline 408 & $10444742-5935081$ & 0.65 & $13.73 \pm 0.03$ & $13.08 \pm 0.03$ & $12.88 \pm 0.03$ & AAA & 000 & 1.65 & 2.37 & \\
\hline 409 & -------- & -- & ----- & ----- & ----- & -- & -- & -- & -- & \\
\hline 410 & -------- & -- & ----- & ----- & ----- & -- & -- & -- & -- & \\
\hline 411 & $10444777-5946384$ & 0.64 & $14.58 \pm 0.05$ & $14.27 \pm 0.06$ & $14.02 \pm 0.06$ & AAA & $\mathrm{ccc}$ & 1.83 & 2.05 & \\
\hline 412 & $10444775-5947176$ & 0.34 & $15.41 \pm 0.09$ & $14.25 \pm 0.05$ & $13.77 \pm 0.06$ & AEA & $\mathrm{ccc}$ & $\mathrm{NaN}$ & 1.39 & \\
\hline 413 & $10444777-5946067$ & 0.32 & $14.23 \pm 0.03$ & $13.44 \pm 0.03$ & $13.12 \pm 0.04$ & AAA & 000 & 3.44 & 2.22 & \\
\hline 414 & $10444774-5946323$ & 0.24 & $14.31 \pm 0.05$ & $13.27 \pm 0.05$ & $12.83 \pm 0.04$ & AAA & 000 & 5.60 & 2.19 & \\
\hline 415 & $10444791-5945397$ & 0.40 & $15.88 \pm 0.11$ & $14.93 \pm 0.09$ & $14.72 \pm 0.14$ & BAB & $\mathrm{ccc}$ & $\mathrm{NaN}$ & 1.10 & \\
\hline 416 & $10444796-5943095$ & 0.27 & $14.74 \pm 0.05$ & $13.88 \pm 0.05$ & $13.57 \pm 0.08$ & AAA & $00 \mathrm{c}$ & 3.18 & 1.95 & \\
\hline 417 & -------- & -- & ----- & ----- & ----- & -- & -- & -- & -- & \\
\hline 418 & $10444891-5938245$ & 0.60 & $15.19 \pm 0.08$ & $14.21 \pm 0.06$ & $13.58 \pm 0.06$ & AAA & 000 & 8.37 & 1.57 & K-excess \\
\hline 419 & $10444898-5943435$ & 0.32 & $13.17 \pm 0.04$ & $12.41 \pm 0.05$ & $12.09 \pm 0.05$ & AEA & $\mathrm{c} 00$ & $\mathrm{NaN}$ & 3.55 & Mass-deg. \\
\hline 420 & $10444902-5940445$ & 0.36 & $12.45 \pm * * * *$ & $11.81 \pm 0.03$ & $11.45 \pm 0.04$ & UAA & $0 \mathrm{cc}$ & $\mathrm{NaN}$ & $\mathrm{NaN}$ & \\
\hline 421 & $10444910-5941350$ & 1.15 & $15.72 \pm * * * *$ & $15.16 \pm 0.12$ & $14.32 \pm * * * *$ & UBU & 0p0 & $\mathrm{NaN}$ & $\mathrm{NaN}$ & \\
\hline 422 & $10444912-5944225$ & 0.11 & $15.79 \pm 0.11$ & $14.75 \pm 0.08$ & $14.28 \pm 0.09$ & AAA & $\mathrm{c} 00$ & 5.47 & 1.15 & \\
\hline 423 & -------- & -- & ----- & ----- & ----- & -- & -- & -- & -- & \\
\hline 424 & $10444924-5947332$ & 0.24 & $14.76 \pm 0.06$ & $13.89 \pm 0.07$ & $13.56 \pm 0.06$ & AAA & 000 & 3.49 & 1.93 & \\
\hline 425 & $10444928-5940405$ & 0.10 & $13.38 \pm * * * *$ & $14.11 \pm 0.09$ & $13.60 \pm 0.11$ & UAB & $0 \mathrm{cc}$ & $\mathrm{NaN}$ & $\mathrm{NaN}$ & \\
\hline 426 & $10444942-5939577$ & 0.40 & $15.38 \pm 0.09$ & $14.17 \pm 0.04$ & $13.85 \pm 0.05$ & AAA & $\mathrm{ccc}$ & 3.13 & 1.41 & \\
\hline 427 & ------- & -- & ----- & ----- & ----- & -- & -- & -- & -- & \\
\hline 428 & -------- & -- & ----- & ----- & ---- & -- & -- & -- & -- & \\
\hline 429 & $10444968-5945032$ & 0.18 & $14.59 \pm * * * *$ & $13.72 \pm * * * *$ & $13.84 \pm 0.06$ & UUA & $00 \mathrm{c}$ & $\mathrm{NaN}$ & $\mathrm{NaN}$ & \\
\hline 430 & $10444974-5946152$ & 0.11 & $15.60 \pm 0.09$ & $14.65 \pm 0.07$ & $14.44 \pm 0.11$ & AAB & $\mathrm{ccc}$ & $\mathrm{NaN}$ & 1.27 & \\
\hline 431 & $10444976-5942081$ & 0.19 & $14.07 \pm 0.03$ & $13.18 \pm 0.03$ & $12.66 \pm 0.04$ & AAA & $\mathrm{c} 0 \mathrm{c}$ & 6.89 & 2.27 & \\
\hline 432 & $10444978-5947273$ & 0.07 & $13.97 \pm 0.04$ & $13.14 \pm 0.04$ & $12.65 \pm 0.04$ & AAA & 000 & 6.41 & 2.30 & \\
\hline 433 & -------- & -- & ----- & ----- & ----- & -- & -- & -- & -- & \\
\hline 434 & -------- & -- & ----- & ----- & ----- & -- & -- & -- & -- & \\
\hline 435 & -------- & -- & ----- & ----- & ----- & -- & -- & -- & -- & \\
\hline 436 & $10445011-5939084$ & 0.23 & $15.29 \pm 0.13$ & $14.46 \pm 0.09$ & $13.90 \pm 0.10$ & BAA & $\mathrm{c} 0 \mathrm{c}$ & $\mathrm{NaN}$ & 1.47 & \\
\hline 437 & $10445005-5938448$ & 0.08 & $15.30 \pm 0.08$ & $14.35 \pm 0.06$ & $13.96 \pm 0.09$ & AAA & $\mathrm{c} 0 \mathrm{c}$ & 4.29 & 1.47 & \\
\hline 438 & $10445008-5944428$ & 0.19 & $15.37 \pm 0.07$ & $14.52 \pm 0.06$ & $14.06 \pm 0.10$ & AAA & $\mathrm{ccc}$ & 5.31 & 1.42 & \\
\hline 439 & $10445009-5945534$ & 0.64 & $14.34 \pm 0.04$ & $13.57 \pm 0.04$ & $13.15 \pm 0.04$ & AAA & 000 & 5.17 & 2.18 & \\
\hline 440 & $10445014-5947386$ & 0.93 & $14.02 \pm 0.04$ & $13.22 \pm 0.04$ & $12.97 \pm 0.05$ & AAA & 000 & 2.49 & 2.28 & \\
\hline 441 & $10445012-5945236$ & 0.34 & $14.52 \pm 0.06$ & $13.70 \pm * * * *$ & $13.32 \pm * * * *$ & AUU & $\mathrm{c} 00$ & $\mathrm{NaN}$ & 2.08 & \\
\hline 442 & -------- & -- & ----- & ----- & ----- & -- & -- & -- & -- & \\
\hline 443 & $10445016-5944104$ & 0.31 & $15.16 \pm 0.06$ & $14.22 \pm 0.04$ & $13.54 \pm 0.04$ & AAA & $\mathrm{c} 00$ & 9.33 & 1.60 & K-excess \\
\hline 444 & $10445020-5947151$ & 0.13 & $15.14 \pm 0.05$ & $14.20 \pm 0.05$ & $13.96 \pm 0.06$ & AAA & 000 & 1.76 & 1.61 & \\
\hline 445 & -------- & -- & ----- & ----- & ----- & -- & -- & -- & -- & \\
\hline 446 & $10445036-5940174$ & 0.37 & $13.32 \pm * * * *$ & $12.56 \pm 0.03$ & $12.32 \pm 0.03$ & UAA & $00 \mathrm{c}$ & $\mathrm{NaN}$ & $\mathrm{NaN}$ & \\
\hline 447 & $10445043-5945408$ & 0.25 & $12.73 \pm 0.03$ & $12.17 \pm 0.04$ & $11.95 \pm 0.03$ & AAA & $\mathrm{ccc}$ & 2.22 & 4.78 & \\
\hline 448 & $10445078-5945276$ & 0.35 & $14.75 \pm 0.05$ & $13.81 \pm 0.04$ & $13.49 \pm 0.05$ & AAE & 000 & $\mathrm{NaN}$ & 1.94 & \\
\hline 449 & $10445081-5944429$ & 0.02 & $16.22 \pm 0.15$ & $15.03 \pm 0.09$ & $14.03 \pm * * * *$ & BAU & $\operatorname{cc} 0$ & $\mathrm{NaN}$ & 0.89 & \\
\hline 450 & -------- & -- & ----- & ----- & ----- & -- & -- & -- & -- & \\
\hline
\end{tabular}


J. F. Albacete-Colombo et al.: An X-ray survey of low-mass stars in Trumpler 16 with Chandra, Online Material p 31

Table 2. continued.

\begin{tabular}{|c|c|c|c|c|c|c|c|c|c|c|}
\hline$N_{x}$ & 2MASS J+ & Off." & $J$ mag & $H \mathrm{mag}$ & $K_{\mathrm{s}} \mathrm{mag}$ & Ph.Q & Cont. & $A_{\mathrm{v}}$ & Mass & ID.flags \\
\hline 451 & $10445091-5944356$ & 0.31 & $14.53 \pm 0.04$ & $13.79 \pm 0.02$ & $13.47 \pm 0.04$ & AAE & 000 & $\mathrm{NaN}$ & 2.07 & \\
\hline 452 & -------- & -- & ----- & ----- & ----- & -- & -- & -- & -- & \\
\hline 453 & $10445112-5939384$ & 0.31 & $14.99 \pm 0.05$ & $13.80 \pm 0.03$ & $13.54 \pm 0.05$ & AAA & $\mathrm{c} 0 \mathrm{c}$ & 2.38 & 1.75 & \\
\hline 454 & $10445108-5944255$ & 0.24 & $16.38 \pm * * * *$ & $15.41 \pm 0.14$ & $15.06 \pm 0.19$ & UBC & $0 \mathrm{cc}$ & $\mathrm{NaN}$ & $\mathrm{NaN}$ & \\
\hline 455 & -------- & -- & ----- & ----- & ----- & -- & -- & -- & -- & \\
\hline 456 & -------- & -- & ----- & ----- & ----- & -- & -- & -- & -- & \\
\hline 457 & $10445162-5948382$ & 0.21 & $15.27 \pm 0.09$ & $13.90 \pm 0.06$ & $13.42 \pm 0.06$ & AAA & $\operatorname{ccc}$ & 5.96 & 1.49 & \\
\hline 458 & $10445134-5944068$ & 0.14 & $15.46 \pm 0.09$ & $14.50 \pm 0.05$ & $14.34 \pm 0.10$ & AAA & $00 \mathrm{c}$ & $\mathrm{NaN}$ & 1.36 & \\
\hline 459 & -------- & -- & ----- & ----- & ----- & -- & -- & -- & -- & \\
\hline 460 & -------- & -- & ----- & ----- & ----- & -- & -- & -- & -- & \\
\hline 461 & $10445156-5950286$ & 0.61 & $12.42 \pm 0.02$ & $12.02 \pm 0.03$ & $11.88 \pm 0.03$ & AAA & 000 & 0.92 & 5.55 & \\
\hline 462 & $10445175-5943055$ & 2.03 & $15.42 \pm 0.08$ & $14.44 \pm 0.06$ & $14.23 \pm 0.09$ & AAA & $\mathrm{ccc}$ & 0.29 & 1.38 & \\
\hline 463 & -------- & -- & ----- & ----- & ----- & -- & -- & -- & -- & \\
\hline 464 & -------- & -- & ----- & ----- & ----- & -- & -- & -- & -- & \\
\hline 465 & -------- & -- & ----- & ----- & ----- & -- & -- & -- & -- & \\
\hline 466 & -------- & -- & ----- & ----- & ----- & -- & -- & -- & -- & \\
\hline 467 & -------- & -- & ----- & ----- & ----- & -- & -- & -- & -- & \\
\hline 468 & $10445224-5941552$ & 0.26 & $12.59 \pm * * * *$ & $12.52 \pm 0.05$ & $11.97 \pm * * * *$ & UAU & $0 \mathrm{c} 0$ & $\mathrm{NaN}$ & $\mathrm{NaN}$ & \\
\hline 469 & -------- & -- & ----- & ----- & ----- & -- & -- & -- & -- & \\
\hline 470 & -------- & -- & ----- & ----- & ----- & -- & -- & -- & -- & \\
\hline 471 & -------- & -- & ----- & ----- & ----- & -- & -- & -- & -- & \\
\hline 472 & $10445267-5951232$ & 1.62 & $17.74 \pm * * * *$ & $15.18 \pm 0.09$ & $14.31 \pm 0.09$ & UAA & $0 \mathrm{cc}$ & $\mathrm{NaN}$ & $\mathrm{NaN}$ & \\
\hline 473 & $10445275-5943190$ & 0.49 & $14.17 \pm 0.04$ & $13.42 \pm 0.04$ & $13.19 \pm 0.04$ & AAA & $00 \mathrm{c}$ & 1.94 & 2.24 & \\
\hline 474 & $10445284-5946342$ & 0.17 & $15.35 \pm 0.07$ & $14.28 \pm 0.05$ & $13.93 \pm 0.07$ & AAA & 000 & 3.73 & 1.43 & \\
\hline 475 & -------- & -- & ----- & ----- & ----- & -- & -- & -- & -- & \\
\hline 476 & -------- & -- & ----- & ----- & ----- & -- & -- & -- & -- & \\
\hline 477 & -------- & -- & ----- & ----- & ----- & -- & -- & -- & -- & \\
\hline 478 & $10445326-5940003$ & 0.17 & $15.74 \pm * * * *$ & $14.99 \pm 0.09$ & $15.35 \pm * * * *$ & UAU & $0 \mathrm{c} 0$ & $\mathrm{NaN}$ & $\mathrm{NaN}$ & \\
\hline 479 & -------- & -- & ----- & ----- & ----- & -- & -- & -- & -- & \\
\hline 480 & -------- & -- & ----- & ----- & ----- & -- & -- & -- & -- & \\
\hline 481 & -------- & -- & ----- & ----- & ----- & -- & -- & -- & -- & \\
\hline 482 & $10445371-5943244$ & 0.28 & $13.01 \pm 0.03$ & $12.46 \pm 0.03$ & $12.19 \pm 0.03$ & AAA & 000 & 2.99 & 3.55 & Mass-deg. \\
\hline 483 & $10445372-5944260$ & 0.41 & $15.52 \pm * * * *$ & $15.00 \pm 0.10$ & $14.56 \pm 0.11$ & UAB & $0 \mathrm{cc}$ & $\mathrm{NaN}$ & $\mathrm{NaN}$ & \\
\hline 484 & -------- & -- & ----- & ----- & ----- & -- & -- & -- & -- & \\
\hline 485 & $10445386-5943140$ & 0.57 & $13.23 \pm 0.04$ & $12.73 \pm 0.05$ & $12.55 \pm 0.05$ & AAA & $\operatorname{ccc}$ & 1.41 & 3.55 & Mass-deg. \\
\hline 486 & $10445393-5945240$ & 0.33 & $12.42 \pm 0.02$ & $12.18 \pm 0.03$ & $12.10 \pm 0.03$ & AAA & 000 & 3.20 & 5.55 & \\
\hline 487 & -------- & -- & ----- & ----- & ----- & -- & -- & -- & -- & \\
\hline 488 & $10445406-5944436$ & 0.26 & $15.88 \pm 0.13$ & $15.25 \pm 0.14$ & $14.86 \pm 0.17$ & $\mathrm{BBC}$ & $\mathrm{ccc}$ & $\mathrm{NaN}$ & 1.10 & \\
\hline 489 & $10445407-5941293$ & 0.15 & $10.21 \pm 0.05$ & $10.15 \pm 0.07$ & $10.16 \pm 0.04$ & AAA & 000 & 0.54 & 13.58 & B1V \\
\hline 490 & -------- & -- & ----- & ----- & ----- & -- & -- & -- & -- & \\
\hline 491 & $10445417-5945364$ & 0.25 & $14.67 \pm 0.05$ & $13.42 \pm 0.04$ & $12.65 \pm 0.04$ & AAA & 000 & 10.88 & 1.99 & K-excess \\
\hline 492 & -------- & -- & ----- & ----- & ----- & -- & -- & -- & -- & \\
\hline 493 & -------- & -- & ----- & ----- & ----- & -- & -- & -- & -- & \\
\hline 494 & -------- & -- & ----- & ----- & ----- & -- & -- & -- & -- & \\
\hline 495 & $10445439-5939251$ & 0.15 & $14.52 \pm 0.04$ & $13.28 \pm 0.04$ & $12.78 \pm 0.04$ & AAA & $\mathrm{c} 0 \mathrm{c}$ & 6.55 & 2.08 & \\
\hline 496 & $10445438-5944239$ & 0.09 & $14.41 \pm * * * *$ & $13.80 \pm 0.05$ & $13.51 \pm 0.06$ & UAA & $0 \mathrm{cc}$ & $\mathrm{NaN}$ & $\mathrm{NaN}$ & \\
\hline 497 & $10445449-5942432$ & 0.57 & $13.85 \pm * * * *$ & $13.30 \pm 0.06$ & $13.08 \pm 0.06$ & UAA & $0 \mathrm{cc}$ & $\mathrm{NaN}$ & $\mathrm{NaN}$ & \\
\hline 498 & $10445467-5944438$ & 0.15 & $15.82 \pm 0.11$ & $14.81 \pm 0.09$ & $14.56 \pm 0.12$ & BAB & $\mathrm{ccc}$ & $\mathrm{NaN}$ & 1.14 & \\
\hline 499 & $10445475-5949232$ & 0.27 & $15.22 \pm 0.08$ & $13.62 \pm 0.05$ & $12.80 \pm * * * *$ & AAU & $\operatorname{cc} 0$ & $\mathrm{NaN}$ & 1.53 & \\
\hline 500 & $10445472-5943392$ & 0.23 & $16.12 \pm 0.17$ & $15.22 \pm 0.14$ & $14.84 \pm * * * *$ & $\mathrm{CBU}$ & $\operatorname{cc} 0$ & $\mathrm{NaN}$ & 0.95 & \\
\hline 501 & $10445476-5936005$ & 0.37 & $15.02 \pm 0.05$ & $14.38 \pm 0.04$ & $14.23 \pm 0.07$ & AAA & 000 & $\mathrm{NaN}$ & 1.73 & \\
\hline 502 & -------- & -- & ----- & ----- & ----- & -- & -- & -- & -- & \\
\hline 503 & $10445482-5943516$ & 0.41 & $13.84 \pm 0.03$ & $13.10 \pm 0.03$ & $12.84 \pm 0.03$ & AAA & $\operatorname{ccc}$ & 2.72 & 2.33 & \\
\hline 504 & $10445485-5945451$ & 0.23 & $15.44 \pm 0.09$ & $13.93 \pm * * * *$ & $14.31 \pm 0.13$ & AUB & $\mathrm{c} 0 \mathrm{c}$ & $\mathrm{NaN}$ & 1.38 & \\
\hline 505 & $10445490-5942580$ & 0.47 & $15.72 \pm 0.10$ & $15.02 \pm 0.09$ & $14.59 \pm 0.12$ & $\mathrm{AAB}$ & $\operatorname{ccc}$ & $\mathrm{NaN}$ & 1.19 & \\
\hline 506 & -------- & -- & ----- & ----- & ----- & -- & -- & -- & -- & \\
\hline 507 & $10445506-5948243$ & 0.27 & $14.06 \pm 0.03$ & $13.20 \pm 0.03$ & $12.88 \pm 0.04$ & AAA & $\mathrm{ccc}$ & 3.61 & 2.27 & \\
\hline 508 & ------- & -- & ----- & ----- & ----- & -- & -- & -- & -- & \\
\hline 509 & $10445527-5946486$ & 0.86 & $15.28 \pm 0.07$ & $14.52 \pm 0.07$ & $14.38 \pm 0.10$ & AAA & $\mathrm{ccc}$ & $\mathrm{NaN}$ & 1.48 & \\
\hline 510 & $10445539-5946297$ & 0.94 & $15.18 \pm 0.06$ & $14.35 \pm 0.05$ & $13.90 \pm 0.07$ & AAA & 000 & 5.49 & 1.57 & \\
\hline 511 & -------- & -- & ----- & ----- & ----- & -- & -- & -- & -- & \\
\hline 512 & -------- & -- & ----- & ----- & ----- & -- & -- & -- & -- & \\
\hline 513 & $10445543-5944535$ & 0.42 & $13.37 \pm 0.03$ & $12.51 \pm 0.03$ & $12.18 \pm 0.03$ & AAA & 000 & 3.94 & 2.47 & \\
\hline 514 & $10445591-5942295$ & 0.37 & $14.70 \pm * * * *$ & $14.06 \pm 0.07$ & $13.42 \pm * * * *$ & UAU & 0p0 & $\mathrm{NaN}$ & $\mathrm{NaN}$ & \\
\hline 515 & $10445595-5944480$ & 0.09 & $14.26 \pm 0.05$ & $13.33 \pm 0.05$ & $12.85 \pm 0.05$ & AAA & 000 & 6.28 & 2.21 & \\
\hline 516 & -------- & -- & ----- & ----- & ----- & -- & -- & -- & -- & \\
\hline
\end{tabular}


J. F. Albacete-Colombo et al.: An X-ray survey of low-mass stars in Trumpler 16 with Chandra, Online Material p 32

Table 2. continued.

\begin{tabular}{|c|c|c|c|c|c|c|c|c|c|c|}
\hline$N_{x}$ & 2MASS J+ & Off." & $J \mathrm{mag}$ & $H$ mag & $K_{\mathrm{s}} \mathrm{mag}$ & Ph.Q & Cont. & $A_{\mathrm{v}}$ & Mass & ID.flags \\
\hline 517 & $10445604-5945547$ & 0.18 & $16.06 \pm * * * *$ & $15.49 \pm 0.17$ & $14.92 \pm * * * *$ & $\overline{\mathrm{UCU}}$ & $0 \mathrm{c} 0$ & $\mathrm{NaN}$ & $\mathrm{NaN}$ & \\
\hline 518 & -------- & -- & ----- & ----- & ----- & -- & -- & -- & -- & \\
\hline 519 & $10445616-5948398$ & 0.23 & $15.94 \pm * * * *$ & $14.89 \pm 0.09$ & $14.22 \pm 0.09$ & UAA & 000 & $\mathrm{NaN}$ & $\mathrm{NaN}$ & \\
\hline 520 & $10445610-5946002$ & 0.31 & $15.96 \pm 0.13$ & $15.09 \pm 0.11$ & $14.74 \pm 0.14$ & BBB & $\operatorname{cc} 0$ & $\mathrm{NaN}$ & 1.05 & \\
\hline 521 & $10445613-5945381$ & 0.32 & $14.36 \pm 0.04$ & $13.62 \pm 0.04$ & $13.28 \pm 0.05$ & AAA & $\mathrm{ccc}$ & 3.75 & 2.17 & \\
\hline 522 & $10445615-5948137$ & 0.36 & $14.84 \pm 0.05$ & $13.75 \pm 0.05$ & $13.33 \pm 0.04$ & AAA & 000 & 5.15 & 1.87 & \\
\hline 523 & -------- & -- & ----- & ----- & ----- & -- & -- & -- & -- & \\
\hline 524 & $10445629-5938309$ & 0.46 & $14.52 \pm 0.09$ & $13.70 \pm 0.07$ & $13.20 \pm 0.08$ & AAE & $\mathrm{ccc}$ & $\mathrm{NaN}$ & 2.08 & \\
\hline 525 & -------- & -- & ----- & ----- & ----- & -- & -- & -- & -- & \\
\hline 526 & -------- & -- & ----- & ----- & ----- & -- & -- & -- & -- & \\
\hline 527 & -------- & -- & ----- & ----- & ----- & -- & -- & -- & -- & \\
\hline 528 & -------- & -- & ----- & ----- & ----- & -- & -- & -- & -- & \\
\hline 529 & $10445679-5944182$ & 0.36 & $15.47 \pm 0.08$ & $14.49 \pm 0.05$ & $14.26 \pm * * * *$ & AAU & $0 \mathrm{c} 0$ & $\mathrm{NaN}$ & 1.35 & \\
\hline 530 & -------- & -- & ----- & ----- & ----- & -- & -- & -- & -- & \\
\hline 531 & -------- & -- & ----- & ----- & ----- & -- & -- & -- & -- & \\
\hline 532 & -------- & -- & ----- & ----- & ----- & -- & -- & -- & -- & \\
\hline 533 & -------- & -- & ----- & ----- & ----- & -- & -- & -- & -- & \\
\hline 534 & $10445706-5938268$ & 0.44 & $11.64 \pm 0.02$ & $11.09 \pm 0.02$ & $10.97 \pm 0.03$ & AAA & 000 & 0.83 & 8.30 & \\
\hline 535 & -------- & -- & ----- & ----- & ----- & -- & -- & -- & -- & \\
\hline 536 & $10445721-5942561$ & 0.20 & $16.06 \pm * * * *$ & $15.37 \pm 0.13$ & $15.03 \pm 0.17$ & UBC & $0 \mathrm{cc}$ & $\mathrm{NaN}$ & $\mathrm{NaN}$ & \\
\hline 537 & -------- & -- & ----- & ----- & ----- & -- & -- & -- & -- & \\
\hline 538 & $10445729-5943120$ & 0.43 & $14.86 \pm 0.05$ & $13.93 \pm 0.04$ & $13.71 \pm 0.05$ & AAA & $\mathrm{c0c}$ & 1.51 & 1.86 & \\
\hline 539 & -------- & -- & ----- & ----- & ----- & -- & -- & -- & -- & \\
\hline 540 & -------- & -- & ----- & ----- & ----- & -- & -- & -- & -- & \\
\hline 541 & -------- & -- & ----- & ----- & ----- & -- & -- & -- & -- & \\
\hline 542 & -------- & -- & ----- & ----- & ----- & -- & -- & -- & -- & \\
\hline 543 & $10445747-5948326$ & 0.18 & $15.79 \pm 0.10$ & $14.20 \pm 0.06$ & $13.50 \pm 0.05$ & AAA & $\mathrm{ccc}$ & 9.81 & 1.15 & \\
\hline 544 & $10445754-5944506$ & 0.21 & $14.96 \pm 0.05$ & $14.23 \pm 0.05$ & $14.00 \pm * * * *$ & AAU & $\mathrm{c} 00$ & $\mathrm{NaN}$ & 1.78 & \\
\hline 545 & -------- & -- & ----- & ----- & ----- & -- & -- & -- & -- & \\
\hline 546 & -------- & -- & ----- & ----- & ----- & -- & -- & -- & -- & \\
\hline 547 & -------- & -- & ----- & ----- & ----- & -- & -- & -- & -- & \\
\hline 548 & $10445773-5947234$ & 0.30 & $15.58 \pm 0.13$ & $14.53 \pm 0.11$ & $13.72 \pm * * * *$ & BAU & $\operatorname{cc} 0$ & $\mathrm{NaN}$ & 1.29 & \\
\hline 549 & $10445770-5937545$ & 0.63 & $16.80 \pm 0.29$ & $15.07 \pm 0.11$ & $14.38 \pm 0.10$ & DAA & $\mathrm{ccc}$ & $\mathrm{NaN}$ & 0.42 & Mass-deg. \\
\hline 550 & $10445777-5947154$ & 0.46 & $14.93 \pm * * * *$ & $15.08 \pm 0.14$ & $13.66 \pm * * * *$ & UBU & 0p0 & $\mathrm{NaN}$ & $\mathrm{NaN}$ & \\
\hline 551 & -------- & -- & ----- & ----- & ----- & -- & -- & -- & -- & \\
\hline 552 & $10445799-5947095$ & 0.09 & $13.35 \pm 0.03$ & $12.58 \pm 0.03$ & $12.27 \pm 0.03$ & AAA & $0 \mathrm{c} 0$ & 3.58 & 2.47 & \\
\hline 553 & -------- & -- & ----- & ----- & ----- & -- & -- & -- & -- & \\
\hline 554 & -------- & -- & ----- & ----- & ----- & -- & -- & -- & -- & \\
\hline 555 & $10445818-5942497$ & 0.48 & $15.07 \pm * * * *$ & $14.62 \pm 0.07$ & $14.31 \pm 0.10$ & UAA & $0 \mathrm{cc}$ & $\mathrm{NaN}$ & $\mathrm{NaN}$ & \\
\hline 556 & -------- & -- & ----- & ----- & ----- & -- & -- & -- & -- & \\
\hline 557 & $10445808-5948397$ & 0.24 & $12.82 \pm * * * *$ & $11.44 \pm 0.03$ & $10.50 \pm * * * *$ & UAU & $0 \mathrm{c} 0$ & $\mathrm{NaN}$ & $\mathrm{NaN}$ & \\
\hline 558 & -------- & -- & ----- & ----- & ----- & -- & -- & -- & -- & \\
\hline 559 & -------- & -- & ----- & ----- & ----- & -- & -- & -- & -- & \\
\hline 560 & $10445852-5944108$ & 0.51 & $15.51 \pm * * * *$ & $15.22 \pm 0.17$ & $14.40 \pm * * * *$ & UCU & $0 \mathrm{c} 0$ & $\mathrm{NaN}$ & $\mathrm{NaN}$ & \\
\hline 561 & -------- & -- & ----- & ----- & ----- & -- & -- & -- & -- & \\
\hline 562 & $10445862-5942461$ & 0.15 & $14.19 \pm * * * *$ & $13.58 \pm 0.05$ & $13.05 \pm 0.05$ & UAA & $0 \mathrm{cc}$ & $\mathrm{NaN}$ & $\mathrm{NaN}$ & \\
\hline 563 & -------- & -- & ----- & ----- & ----- & -- & -- & -- & -- & \\
\hline 564 & $10445857-5950232$ & 0.87 & $16.23 \pm 0.14$ & $14.63 \pm 0.06$ & $13.70 \pm 0.05$ & BAA & 000 & $\mathrm{NaN}$ & 0.89 & \\
\hline 565 & -------- & -- & ----- & ----- & ----- & -- & -- & -- & -- & \\
\hline 566 & -------- & -- & ----- & ----- & ----- & -- & -- & -- & -- & \\
\hline 567 & -------- & -- & ----- & ----- & ----- & -- & -- & -- & -- & \\
\hline 568 & -------- & -- & ----- & ----- & ----- & -- & -- & -- & -- & \\
\hline 569 & $10445928-5944594$ & 0.21 & $14.47 \pm * * * *$ & $13.72 \pm 0.05$ & $13.36 \pm * * * *$ & UAU & $0 \mathrm{c} 0$ & $\mathrm{NaN}$ & $\mathrm{NaN}$ & \\
\hline 570 & -------- & -- & ----- & ----- & ----- & -- & -- & -- & -- & \\
\hline 571 & -------- & -- & ----- & ----- & ----- & -- & -- & -- & -- & \\
\hline 572 & -------- & -- & ----- & ----- & ----- & -- & -- & -- & -- & \\
\hline 573 & $10445943-5946500$ & 0.16 & $15.59 \pm 0.09$ & $14.69 \pm 0.06$ & $14.45 \pm 0.11$ & $\mathrm{AAB}$ & $0 \mathrm{c} 0$ & $\mathrm{NaN}$ & 1.28 & \\
\hline 574 & -------- & -- & ----- & ----- & ----- & -- & -- & -- & -- & \\
\hline 575 & -------- & -- & ----- & ----- & ----- & -- & -- & -- & -- & \\
\hline 576 & -------- & -- & ----- & ----- & ----- & -- & -- & -- & -- & \\
\hline 577 & -------- & -- & ----- & ----- & ----- & -- & -- & -- & -- & \\
\hline 578 & -------- & -- & ----- & ----- & ----- & -- & -- & -- & -- & \\
\hline 579 & -------- & -- & ----- & ----- & ----- & -- & -- & -- & -- & \\
\hline 580 & $10445990-5943149$ & 0.59 & $10.86 \pm 0.02$ & $10.66 \pm 0.03$ & $10.51 \pm 0.02$ & AAA & 000 & 3.03 & 11.16 & \\
\hline 581 & -------- & -- & ----- & ----- & ----- & -- & -- & -- & -- & \\
\hline
\end{tabular}


J. F. Albacete-Colombo et al.: An X-ray survey of low-mass stars in Trumpler 16 with Chandra, Online Material p 33

Table 2. continued.

\begin{tabular}{|c|c|c|c|c|c|c|c|c|c|c|}
\hline$N_{x}$ & 2MASS J+ & Off." & $J$ mag & $H$ mag & $K_{\mathrm{s}} \mathrm{mag}$ & Ph.Q & Cont. & $A_{\mathrm{v}}$ & Mass & ID.flags \\
\hline 582 & $10450009-5947019$ & 0.26 & $14.57 \pm 0.06$ & $13.16 \pm 0.06$ & $12.32 \pm 0.06$ & $\overline{\mathrm{AAA}}$ & 000 & 12.24 & 2.05 & K-excess \\
\hline 583 & -------- & -- & ----- & ----- & ----- & -- & -- & -- & -- & \\
\hline 584 & $10450016-5944036$ & 0.27 & $15.71 \pm 0.10$ & $14.87 \pm 0.09$ & $14.48 \pm 0.12$ & $\mathrm{AAB}$ & $\mathrm{ccc}$ & $\mathrm{NaN}$ & 1.20 & \\
\hline 585 & -------- & -- & ----- & ----- & ----- & -- & -- & -- & -- & \\
\hline 586 & $10450021-5943531$ & 0.20 & $16.16 \pm 0.15$ & $15.30 \pm 0.15$ & $14.87 \pm * * * *$ & BBU & $\operatorname{cc} 0$ & $\mathrm{NaN}$ & 0.93 & \\
\hline 587 & $10450031-5943399$ & 0.33 & $13.99 \pm 0.04$ & $13.20 \pm 0.06$ & $12.92 \pm 0.12$ & AAE & $\mathrm{ccc}$ & $\mathrm{NaN}$ & 2.29 & \\
\hline 588 & $10450045-5943252$ & 0.13 & $15.60 \pm 0.13$ & $14.69 \pm 0.12$ & $14.56 \pm 0.17$ & $\mathrm{BBC}$ & $\mathrm{ccc}$ & $\mathrm{NaN}$ & 1.27 & \\
\hline 589 & -------- & -- & ----- & ----- & ----- & -- & -- & -- & -- & \\
\hline 590 & -------- & -- & ----- & ----- & ----- & -- & -- & -- & -- & \\
\hline 591 & -------- & -- & ----- & ----- & ----- & -- & -- & -- & -- & \\
\hline 592 & -------- & -- & ----- & ----- & ----- & -- & -- & -- & -- & \\
\hline 593 & $10450059-5946035$ & 0.41 & $14.36 \pm 0.04$ & $13.75 \pm 0.05$ & $13.42 \pm 0.07$ & AAA & 000 & 3.53 & 2.17 & \\
\hline 594 & $10450069-5949173$ & 0.24 & $16.56 \pm * * * *$ & $14.71 \pm 0.08$ & $13.43 \pm 0.04$ & UAA & 000 & $\mathrm{NaN}$ & $\mathrm{NaN}$ & \\
\hline 595 & $10450071-5944506$ & 0.15 & $15.72 \pm 0.10$ & $14.81 \pm 0.08$ & $14.72 \pm 0.14$ & AAB & $00 \mathrm{c}$ & $\mathrm{NaN}$ & 1.19 & \\
\hline 596 & $10450071-5945324$ & 0.35 & $16.66 \pm 0.21$ & $15.39 \pm 0.14$ & $15.92 \pm * * * *$ & $\mathrm{CBU}$ & 000 & $\mathrm{NaN}$ & 0.42 & Mass-deg. \\
\hline 597 & $10450089-5942451$ & 0.35 & $15.58 \pm * * * *$ & $14.99 \pm 0.11$ & $14.42 \pm * * * *$ & UBU & 0c0 & $\mathrm{NaN}$ & $\mathrm{NaN}$ & \\
\hline 598 & $10450089-5947190$ & 0.28 & $17.30 \pm * * * *$ & $14.40 \pm 0.05$ & $12.33 \pm 0.02$ & UAA & 000 & $\mathrm{NaN}$ & $\mathrm{NaN}$ & \\
\hline 599 & -------- & -- & ----- & ----- & ----- & -- & -- & -- & -- & \\
\hline 600 & $10450102-5945156$ & 0.42 & $12.60 \pm 0.02$ & $12.12 \pm 0.03$ & $12.03 \pm 0.03$ & AAA & sss & 0.14 & 5.05 & \\
\hline 601 & -------- & -- & ----- & ----- & ----- & -- & -- & -- & -- & \\
\hline 602 & -------- & -- & ----- & ----- & ----- & -- & -- & -- & -- & \\
\hline 603 & $10450111-5939176$ & 0.11 & $13.90 \pm * * * *$ & $13.34 \pm 0.14$ & $13.18 \pm 0.17$ & UBC & $0 \mathrm{cc}$ & $\mathrm{NaN}$ & $\mathrm{NaN}$ & \\
\hline 604 & $10450109-5938531$ & 0.60 & $14.70 \pm 0.10$ & $14.07 \pm 0.09$ & $13.75 \pm 0.13$ & $\mathrm{AAB}$ & $\mathrm{ccc}$ & $\mathrm{NaN}$ & 1.97 & \\
\hline 605 & $10450115-5944153$ & 0.20 & $15.06 \pm 0.06$ & $14.21 \pm 0.07$ & $13.80 \pm 0.07$ & AAA & $00 \mathrm{c}$ & 4.75 & 1.69 & \\
\hline 606 & $10450118-5938222$ & 0.16 & $15.14 \pm 0.09$ & $14.07 \pm 0.09$ & $13.72 \pm 0.07$ & AAA & $\mathrm{c} 0 \mathrm{c}$ & 3.77 & 1.62 & \\
\hline 607 & -------- & -- & ----- & ----- & ----- & -- & -- & -- & -- & \\
\hline 608 & -------- & -- & ----- & ----- & ----- & -- & -- & -- & -- & \\
\hline 609 & $10450145-5946338$ & 0.82 & $14.44 \pm 0.05$ & $13.35 \pm * * * *$ & $12.85 \pm * * * *$ & AUU & $\mathrm{c} 00$ & $\mathrm{NaN}$ & 2.12 & \\
\hline 610 & -------- & -- & ----- & ----- & ----- & -- & -- & -- & -- & \\
\hline 611 & -------- & -- & ----- & ----- & ----- & -- & -- & -- & -- & \\
\hline 612 & -------- & -- & ----- & ----- & ----- & -- & -- & -- & -- & \\
\hline 613 & -------- & -- & ----- & ----- & ----- & -- & -- & -- & -- & \\
\hline 614 & -------- & -- & ----- & ----- & ----- & -- & -- & -- & -- & \\
\hline 615 & -------- & -- & ----- & ----- & ----- & -- & -- & -- & -- & \\
\hline 616 & $10450168-5947102$ & 0.32 & $17.26 \pm * * * *$ & $15.86 \pm * * * *$ & $14.68 \pm 0.15$ & UUB & 000 & $\mathrm{NaN}$ & $\mathrm{NaN}$ & \\
\hline 617 & $10450193-5942371$ & 0.47 & $14.79 \pm * * * *$ & $14.70 \pm 0.08$ & $13.35 \pm * * * *$ & UAU & 0c0 & $\mathrm{NaN}$ & $\mathrm{NaN}$ & \\
\hline 618 & -------- & -- & ----- & ----- & ----- & -- & -- & -- & -- & \\
\hline 619 & -------- & -- & ----- & ----- & ----- & -- & -- & -- & -- & \\
\hline 620 & -------- & -- & ----- & ----- & ----- & -- & -- & -- & -- & \\
\hline 621 & $10450201-5945317$ & 0.32 & $14.29 \pm 0.06$ & $13.27 \pm 0.07$ & $12.54 \pm 0.05$ & AAA & $\operatorname{ccc}$ & 10.31 & 2.20 & K-excess \\
\hline 622 & -------- & -- & ----- & ----- & ----- & -- & -- & -- & -- & \\
\hline 623 & $10450208-5937439$ & 1.00 & $15.30 \pm 0.06$ & $14.35 \pm 0.04$ & $14.09 \pm 0.07$ & AAA & $00 \mathrm{c}$ & 1.84 & 1.47 & \\
\hline 624 & -------- & -- & ----- & ----- & ----- & -- & -- & -- & -- & \\
\hline 625 & $10450211-5943212$ & 0.83 & $16.26 \pm 0.17$ & $15.22 \pm 0.13$ & $14.72 \pm * * * *$ & $\mathrm{CBU}$ & $\operatorname{cc} 0$ & $\mathrm{NaN}$ & 0.87 & \\
\hline 626 & -------- & -- & ----- & ----- & ----- & -- & -- & -- & -- & \\
\hline 627 & -------- & -- & ----- & ----- & ----- & -- & -- & -- & -- & \\
\hline 628 & -------- & -- & ----- & ----- & ----- & -- & -- & -- & -- & \\
\hline 629 & $10450220-5942492$ & 0.16 & $15.35 \pm * * * *$ & $14.02 \pm 0.05$ & $13.72 \pm 0.05$ & UAA & $0 \mathrm{cc}$ & $\mathrm{NaN}$ & $\mathrm{NaN}$ & \\
\hline 630 & $10450220-5943468$ & 0.14 & $16.15 \pm * * * *$ & $14.81 \pm * * * *$ & $14.21 \pm 0.09$ & UUA & $00 \mathrm{c}$ & $\mathrm{NaN}$ & $\mathrm{NaN}$ & \\
\hline 631 & $10450225-5945363$ & 0.37 & $14.57 \pm 0.04$ & $13.61 \pm 0.05$ & $13.31 \pm 0.05$ & AAA & $\mathrm{ccc}$ & 3.07 & 2.05 & \\
\hline 632 & $10450243-5945410$ & 0.28 & $13.25 \pm 0.03$ & $12.83 \pm 0.04$ & $12.60 \pm 0.04$ & AAA & $\operatorname{ccc}$ & 2.13 & 3.55 & Mass-deg. \\
\hline 633 & -------- & -- & ----- & ----- & ----- & -- & -- & -- & -- & \\
\hline 634 & -------- & -- & ----- & ----- & ----- & -- & -- & -- & -- & \\
\hline 635 & -------- & -- & ----- & ----- & ----- & -- & -- & -- & -- & \\
\hline 636 & -------- & -- & ----- & ----- & ----- & -- & -- & -- & -- & \\
\hline 637 & -------- & -- & ----- & ----- & ----- & -- & -- & -- & -- & \\
\hline 638 & -------- & -- & ----- & ----- & ----- & -- & -- & -- & -- & \\
\hline 639 & -------- & -- & ----- & ----- & ----- & -- & -- & -- & -- & \\
\hline 640 & -------- & -- & ----- & ----- & ----- & -- & -- & -- & -- & \\
\hline 641 & -------- & -- & ----- & ----- & ----- & -- & -- & -- & -- & \\
\hline 642 & -------- & -- & ----- & ----- & ----- & -- & -- & -- & -- & \\
\hline 643 & -------- & -- & ----- & ----- & ----- & -- & -- & -- & -- & \\
\hline 644 & -------- & -- & ----- & ----- & ----- & -- & -- & -- & -- & \\
\hline 645 & $10450343-5946382$ & 0.37 & $14.04 \pm 0.04$ & $13.09 \pm 0.03$ & $12.66 \pm 0.05$ & AAA & $\mathrm{ccc}$ & 5.46 & 2.28 & \\
\hline 646 & -------- & -- & ----- & ----- & ----- & -- & -- & -- & -- & \\
\hline 647 & -------- & -- & ----- & ----- & ----- & -- & -- & -- & -- & \\
\hline 648 & -------- & -- & ----- & ----- & ----- & -- & -- & -- & -- & \\
\hline
\end{tabular}


J. F. Albacete-Colombo et al.: An X-ray survey of low-mass stars in Trumpler 16 with Chandra, Online Material p 34

Table 2. continued.

\begin{tabular}{|c|c|c|c|c|c|c|c|c|c|c|}
\hline$\overline{\bar{N}} N_{x}$ & 2MASS J+ & Off." & $J$ mag & $H \mathrm{mag}$ & $K_{\mathrm{s}} \mathrm{mag}$ & Ph.Q & Cont. & $\bar{A} A_{\mathrm{v}}$ & Mass & ID.flags \\
\hline 649 & $10450360-5941040$ & 0.17 & $1.66 \pm 0.53$ & $1.07 \pm 0.40$ & $0.17 \pm 0.38$ & $\overline{\mathrm{DDD}}$ & 000 & $\mathrm{NaN}$ & 45.25 & LBV $(\eta$-Car) \\
\hline 650 & -------- & -- & ----- & ----- & ----- & -- & -- & -- & -- & \\
\hline 651 & -------- & -- & ----- & ----- & ----- & -- & -- & -- & -- & \\
\hline 652 & -------- & -- & ----- & ----- & ----- & -- & -- & -- & -- & \\
\hline 653 & -------- & -- & ----- & ----- & ----- & -- & -- & -- & -- & \\
\hline 654 & -------- & -- & ----- & ----- & ----- & -- & -- & -- & -- & \\
\hline 655 & -------- & -- & ----- & ----- & ----- & -- & -- & -- & -- & \\
\hline 656 & -------- & -- & ----- & ----- & ----- & -- & -- & -- & -- & \\
\hline 657 & -------- & -- & ----- & ----- & ----- & -- & -- & -- & -- & \\
\hline 658 & -------- & -- & ----- & ----- & ----- & -- & -- & -- & -- & \\
\hline 659 & -------- & -- & ----- & ----- & ----- & -- & -- & -- & -- & \\
\hline 660 & -------- & -- & ----- & ----- & ----- & -- & -- & -- & -- & \\
\hline 661 & -------- & -- & ----- & ----- & ----- & -- & -- & -- & -- & \\
\hline 662 & $10450474-5946142$ & 0.23 & $14.57 \pm 0.04$ & $13.56 \pm 0.03$ & $13.32 \pm 0.04$ & AAA & $\mathrm{ccc}$ & 2.15 & 2.05 & \\
\hline 663 & -------- & -- & ----- & ----- & ----- & -- & -- & -- & -- & \\
\hline 664 & $10450477-5946091$ & 0.32 & $15.79 \pm 0.11$ & $14.76 \pm 0.08$ & $14.80 \pm 0.15$ & $\mathrm{AAB}$ & $\mathrm{ccc}$ & $\mathrm{NaN}$ & 1.15 & \\
\hline 665 & $10450477-5950041$ & 0.39 & $13.32 \pm 0.03$ & $12.03 \pm 0.05$ & $11.36 \pm 0.04$ & AAA & $\mathrm{ccc}$ & $\mathrm{NaN}$ & 2.48 & \\
\hline 666 & -------- & -- & ----- & ----- & ----- & -- & -- & -- & -- & \\
\hline 667 & -------- & -- & ----- & ----- & ----- & -- & -- & -- & -- & \\
\hline 668 & -------- & -- & ----- & ----- & ----- & -- & -- & -- & -- & \\
\hline 669 & -------- & -- & ----- & ----- & ----- & -- & -- & -- & -- & \\
\hline 670 & $10450506-5937335$ & 0.24 & $13.15 \pm 0.04$ & $12.63 \pm 0.03$ & $12.48 \pm 0.04$ & $\mathrm{AAA}$ & $\mathrm{c} 0 \mathrm{c}$ & 0.97 & 3.55 & Mass-deg. \\
\hline 671 & $10450506-5945311$ & 0.50 & $14.49 \pm * * * *$ & $15.01 \pm 0.12$ & $14.55 \pm 0.12$ & UBB & $0 \mathrm{cc}$ & $\mathrm{NaN}$ & $\mathrm{NaN}$ & \\
\hline 672 & -------- & -- & ----- & ----- & ----- & -- & -- & -- & -- & \\
\hline 673 & -------- & -- & ----- & ----- & ----- & -- & -- & -- & -- & \\
\hline 674 & $10450526-5945400$ & 0.77 & $15.42 \pm 0.09$ & $14.24 \pm 0.06$ & $13.96 \pm 0.08$ & AAA & $\mathrm{ccc}$ & 2.47 & 1.39 & \\
\hline 675 & $10450534-5945452$ & 0.24 & $14.50 \pm 0.04$ & $13.12 \pm 0.03$ & $12.56 \pm 0.03$ & AAA & $\mathrm{ccc}$ & 7.55 & 2.09 & \\
\hline 676 & -------- & -- & ----- & ----- & ----- & -- & -- & -- & -- & \\
\hline 677 & -------- & -- & ----- & ----- & ----- & -- & -- & -- & -- & \\
\hline 678 & $10450541-5944391$ & 0.49 & $16.66 \pm 0.23$ & $15.14 \pm 0.11$ & $14.92 \pm 0.17$ & $\mathrm{DBC}$ & $\mathrm{c} 0 \mathrm{c}$ & $\mathrm{NaN}$ & 0.42 & Mass-deg. \\
\hline 679 & -------- & -- & ----- & ----- & ----- & -- & -- & -- & -- & \\
\hline 680 & $10450559-5945307$ & 0.18 & $13.64 \pm * * * *$ & $13.25 \pm 0.06$ & $12.94 \pm 0.07$ & UAA & $0 \mathrm{cc}$ & $\mathrm{NaN}$ & $\mathrm{NaN}$ & \\
\hline 681 & $10450563-5944478$ & 0.72 & $15.07 \pm 0.06$ & $13.96 \pm 0.05$ & $13.32 \pm 0.07$ & AAA & $\mathrm{ccc}$ & 8.76 & 1.68 & K-excess \\
\hline 682 & -------- & -- & ----- & ----- & ----- & -- & -- & -- & -- & \\
\hline 683 & $10450572-5946067$ & 0.11 & $14.84 \pm 0.05$ & $13.89 \pm 0.04$ & $13.46 \pm 0.05$ & AAA & $\operatorname{cd} 0$ & 5.25 & 1.88 & \\
\hline 684 & -------- & -- & ----- & ----- & ----- & -- & -- & -- & -- & \\
\hline 685 & -------- & -- & ----- & ----- & ----- & -- & -- & -- & -- & \\
\hline 686 & $10450575-5944053$ & 0.11 & $15.45 \pm 0.09$ & $14.57 \pm 0.06$ & $14.31 \pm 0.09$ & AAA & $\mathrm{c} 0 \mathrm{c}$ & 0.42 & 1.37 & \\
\hline 687 & $10450579-5945195$ & 0.15 & $8.65 \pm 0.02$ & $8.42 \pm 0.02$ & $8.34 \pm 0.02$ & AAA & sss & 2.08 & 19.36 & $\mathrm{O} 7 \mathrm{~V}$ \\
\hline 688 & $10450584-5943077$ & 0.24 & $8.92 \pm 0.01$ & $8.80 \pm 0.02$ & $8.76 \pm 0.02$ & AAA & 000 & 1.40 & 18.37 & $09.5 \mathrm{~V}$ \\
\hline 689 & $10450592-5940061$ & 0.67 & $7.71 \pm 0.01$ & $7.71 \pm 0.03$ & $7.62 \pm 0.05$ & AAA & Odd & 2.21 & 22.85 & $\mathrm{O} 4 \mathrm{~V}((\mathrm{f}))$ \\
\hline 690 & -------- & -- & ----- & ----- & ----- & -- & -- & -- & -- & \\
\hline 691 & -------- & -- & ----- & ----- & ----- & -- & -- & -- & -- & \\
\hline 692 & -------- & -- & ----- & ----- & ----- & -- & -- & -- & -- & \\
\hline 693 & $10450597-5946303$ & 0.34 & $15.31 \pm 0.10$ & $14.33 \pm 0.07$ & $13.89 \pm 0.07$ & AAA & 000 & 5.31 & 1.46 & \\
\hline 694 & $10450605-5944347$ & 0.27 & $15.67 \pm 0.09$ & $14.49 \pm 0.06$ & $14.22 \pm 0.09$ & AAA & $\mathrm{c} 0 \mathrm{c}$ & 0.01 & 1.23 & \\
\hline 695 & $10450609-5946208$ & 0.14 & $14.78 \pm * * * *$ & $14.46 \pm 0.07$ & $14.42 \pm 0.12$ & UAB & $0 \mathrm{cc}$ & $\mathrm{NaN}$ & $\mathrm{NaN}$ & \\
\hline 696 & -------- & -- & ----- & ----- & ----- & -- & -- & -- & -- & \\
\hline 697 & -------- & -- & ----- & ----- & ----- & -- & -- & -- & -- & \\
\hline 698 & -------- & -- & ----- & ----- & ----- & -- & -- & -- & -- & \\
\hline 699 & $10450628-5949455$ & 0.34 & $17.99 \pm * * * *$ & $15.10 \pm 0.09$ & $13.89 \pm 0.06$ & UAA & 000 & $\mathrm{NaN}$ & $\mathrm{NaN}$ & \\
\hline 700 & $10450641-5935094$ & 0.28 & $15.17 \pm 0.07$ & $14.41 \pm 0.06$ & $14.20 \pm 0.10$ & AAA & 000 & 0.39 & 1.59 & \\
\hline 701 & -------- & -- & ----- & ----- & ----- & -- & -- & -- & -- & \\
\hline 702 & $10450630-5944399$ & 0.15 & $16.39 \pm 0.20$ & $15.30 \pm * * * *$ & $14.99 \pm * * * *$ & CUU & $\mathrm{c} 00$ & $\mathrm{NaN}$ & 0.77 & \\
\hline 703 & -------- & -- & ----- & ----- & ----- & -- & -- & -- & -- & \\
\hline 704 & $10450654-5943443$ & 0.51 & $13.88 \pm * * * *$ & $14.42 \pm 0.13$ & $14.15 \pm 0.11$ & UBB & $0 \mathrm{cc}$ & $\mathrm{NaN}$ & $\mathrm{NaN}$ & \\
\hline 705 & $10450661-5943549$ & 1.49 & $14.98 \pm 0.07$ & $14.34 \pm 0.09$ & $14.04 \pm 0.10$ & AAA & $00 \mathrm{c}$ & 2.52 & 1.76 & \\
\hline 706 & -------- & -- & ----- & ----- & ----- & -- & -- & -- & -- & \\
\hline 707 & $10450672-5941565$ & 0.69 & $9.45 \pm 0.01$ & $9.41 \pm 0.03$ & $9.36 \pm 0.03$ & AAA & 000 & 1.62 & 16.39 & $08.5 \mathrm{~V}$ \\
\hline 708 & $10450673-5943287$ & 0.73 & $13.82 \pm 0.02$ & $12.93 \pm 0.02$ & $12.34 \pm 0.02$ & AAA & 000 & 8.13 & 2.34 & K-excess \\
\hline 709 & $10450680-5944460$ & 0.72 & $12.76 \pm 0.03$ & $12.14 \pm 0.04$ & $11.87 \pm 0.04$ & AAA & 000 & 2.99 & 4.72 & \\
\hline 710 & $10450678-5945373$ & 0.10 & $16.23 \pm * * * *$ & $15.08 \pm 0.10$ & $14.72 \pm 0.16$ & UAC & $0 \mathrm{cc}$ & $\mathrm{NaN}$ & $\mathrm{NaN}$ & \\
\hline 711 & -------- & -- & ----- & ----- & ----- & -- & -- & -- & -- & \\
\hline 712 & $10450704-5946162$ & 0.21 & $14.98 \pm 0.07$ & $14.33 \pm 0.06$ & $13.98 \pm 0.08$ & AAA & $\operatorname{ccc}$ & 3.63 & 1.76 & \\
\hline 713 & -------- & -- & ----- & ----- & ----- & -- & -- & -- & -- & \\
\hline 714 & -------- & -- & ----- & ----- & ----- & -- & -- & -- & -- & \\
\hline 715 & $10450709-5943132$ & 0.15 & $15.71 \pm 0.10$ & $14.79 \pm 0.09$ & $14.48 \pm 0.11$ & AAA & $\mathrm{ccc}$ & 0.18 & 1.20 & \\
\hline
\end{tabular}


J. F. Albacete-Colombo et al.: An X-ray survey of low-mass stars in Trumpler 16 with Chandra, Online Material p 35

Table 2. continued.

\begin{tabular}{|c|c|c|c|c|c|c|c|c|c|c|}
\hline$N_{x}$ & 2MASS J+ & Off." & $J$ mag & $H \mathrm{mag}$ & $K_{\mathrm{s}} \mathrm{mag}$ & Ph.Q & Cont. & $A_{\mathrm{v}}$ & Mass & ID.flags \\
\hline 716 & $10450707-5942381$ & 0.40 & $13.36 \pm * * * *$ & $14.65 \pm 0.10$ & $12.99 \pm * * * *$ & $\overline{\mathrm{UAU}}$ & $0 \mathrm{c} 0$ & $\mathrm{NaN}$ & $\mathrm{NaN}$ & \\
\hline 717 & -------- & -- & ----- & ----- & ----- & -- & -- & -- & -- & \\
\hline 718 & -------- & -- & ----- & ----- & ----- & -- & -- & -- & -- & \\
\hline 719 & -------- & -- & ----- & ----- & ----- & -- & -- & -- & -- & \\
\hline 720 & -------- & -- & ----- & ----- & ----- & -- & -- & -- & -- & \\
\hline 721 & -------- & -- & ----- & ----- & ----- & -- & -- & -- & -- & \\
\hline 722 & -------- & -- & ----- & ----- & ----- & -- & -- & -- & -- & \\
\hline 723 & -------- & -- & ----- & ----- & ----- & -- & -- & -- & -- & \\
\hline 724 & $10450782-5950434$ & 1.19 & $14.66 \pm 0.05$ & $13.55 \pm 0.03$ & $12.98 \pm 0.03$ & AAA & 000 & 7.67 & 2.00 & \\
\hline 725 & -------- & -- & ----- & ----- & ----- & -- & -- & -- & -- & \\
\hline 726 & $10450795-5937253$ & 0.21 & $14.66 \pm * * * *$ & $13.66 \pm 0.04$ & $13.34 \pm * * * *$ & UAU & $0 \mathrm{c} 0$ & $\mathrm{NaN}$ & $\mathrm{NaN}$ & \\
\hline 727 & -------- & -- & ----- & ----- & ----- & -- & -- & -- & -- & \\
\hline 728 & $10450807-5945560$ & 0.31 & $15.92 \pm 0.22$ & $14.64 \pm * * * *$ & $14.32 \pm * * * *$ & DUU & $\mathrm{c} 00$ & $\mathrm{NaN}$ & 1.07 & \\
\hline 729 & $10450822-5940494$ & 0.57 & $9.25 \pm 0.02$ & $9.14 \pm 0.02$ & $9.25 \pm 0.06$ & AAA & $00 \mathrm{c}$ & $\mathrm{NaN}$ & 17.14 & B $0.3 \mathrm{~V}$ \\
\hline 730 & $10450822-5946069$ & 0.61 & $9.27 \pm 0.02$ & $8.96 \pm 0.02$ & $8.81 \pm 0.02$ & AAA & 000 & 3.13 & 17.06 & $08.5 \mathrm{~V}$ \\
\hline 731 & $10450836-5938475$ & 0.84 & $11.43 \pm 0.02$ & $11.12 \pm 0.03$ & $11.02 \pm 0.03$ & AAA & 000 & 0.46 & 9.07 & \\
\hline 732 & $10450852-5947043$ & 0.34 & $17.59 \pm * * * *$ & $14.90 \pm 0.09$ & $14.11 \pm 0.08$ & UAA & 000 & $\mathrm{NaN}$ & $\mathrm{NaN}$ & \\
\hline 733 & $10450858-5946140$ & 0.28 & $15.20 \pm 0.14$ & $14.42 \pm 0.12$ & $13.89 \pm 0.11$ & BBA & $\operatorname{ccc}$ & $\mathrm{NaN}$ & 1.55 & \\
\hline 734 & -------- & -- & ----- & ----- & ----- & -- & -- & -- & -- & \\
\hline 735 & $10450858-5943542$ & 0.47 & $15.50 \pm 0.07$ & $14.61 \pm 0.05$ & $14.19 \pm 0.08$ & AAA & $\mathrm{c} 0 \mathrm{c}$ & 4.71 & 1.34 & \\
\hline 736 & -------- & -- & ----- & ----- & ----- & -- & -- & -- & -- & \\
\hline 737 & -------- & -- & ----- & ----- & ----- & -- & -- & -- & -- & \\
\hline 738 & $10450878-5944236$ & 0.35 & $14.39 \pm * * * *$ & $13.60 \pm 0.05$ & $13.24 \pm 0.06$ & UAA & $0 \mathrm{cc}$ & $\mathrm{NaN}$ & $\mathrm{NaN}$ & \\
\hline 739 & -------- & -- & ----- & ----- & ----- & -- & -- & -- & -- & \\
\hline 740 & -------- & -- & ----- & ----- & ----- & -- & -- & -- & -- & \\
\hline 741 & $10450893-5946302$ & 0.41 & $15.92 \pm * * * *$ & $15.23 \pm 0.13$ & $14.94 \pm * * * *$ & UBU & 000 & $\mathrm{NaN}$ & $\mathrm{NaN}$ & \\
\hline 742 & -------- & -- & ----- & ----- & ----- & -- & -- & -- & -- & \\
\hline 743 & $10450891-5942350$ & 0.40 & $15.66 \pm * * * *$ & $14.70 \pm 0.08$ & $14.32 \pm * * * *$ & UAU & 0c0 & $\mathrm{NaN}$ & $\mathrm{NaN}$ & \\
\hline 744 & -------- & -- & ----- & ----- & ----- & -- & -- & -- & -- & \\
\hline 745 & -------- & -- & ----- & ----- & ----- & -- & -- & -- & -- & \\
\hline 746 & $10450902-5942558$ & 0.20 & $13.04 \pm * * * *$ & $14.76 \pm 0.12$ & $14.39 \pm 0.11$ & UBB & $0 \mathrm{cc}$ & $\mathrm{NaN}$ & $\mathrm{NaN}$ & \\
\hline 747 & -------- & -- & ----- & ----- & ----- & -- & -- & -- & -- & \\
\hline 748 & $10450923-5938519$ & 0.18 & $14.50 \pm 0.06$ & $13.82 \pm 0.05$ & $13.53 \pm 0.05$ & AAA & $\mathrm{ccc}$ & 2.82 & 2.09 & \\
\hline 749 & $10450923-5947117$ & 0.26 & $16.27 \pm 0.12$ & $14.39 \pm 0.05$ & $13.54 \pm 0.05$ & BAA & 000 & $\mathrm{NaN}$ & 0.86 & \\
\hline 750 & -------- & -- & ----- & ----- & ----- & -- & -- & -- & -- & \\
\hline 751 & -------- & -- & ----- & ----- & ----- & -- & -- & -- & -- & \\
\hline 752 & $10450949-5945101$ & 0.03 & $15.81 \pm 0.12$ & $14.83 \pm 0.09$ & $14.29 \pm 0.10$ & BAA & $\mathrm{ccc}$ & $\mathrm{NaN}$ & 1.14 & \\
\hline 753 & -------- & -- & ----- & ----- & ----- & -- & -- & -- & -- & \\
\hline 754 & $10450972-5945448$ & 0.59 & $13.01 \pm 0.04$ & $12.60 \pm 0.03$ & $12.33 \pm 0.03$ & AAA & 000 & 2.86 & 3.55 & Mass-deg. \\
\hline 755 & $10450971-5939052$ & 0.26 & $14.94 \pm 0.06$ & $14.10 \pm 0.03$ & $13.91 \pm 0.07$ & AAA & $\operatorname{ccc}$ & 0.69 & 1.80 & \\
\hline 756 & -------- & -- & ----- & ----- & ----- & -- & -- & -- & -- & \\
\hline 757 & $10450977-5945376$ & 0.21 & $17.03 \pm * * * *$ & $15.28 \pm 0.11$ & $14.98 \pm 0.17$ & UBC & $0 \mathrm{cc}$ & $\mathrm{NaN}$ & $\mathrm{NaN}$ & \\
\hline 758 & $10450990-5942335$ & 0.16 & $14.43 \pm * * * *$ & $14.49 \pm 0.10$ & $13.12 \pm * * * *$ & UAU & $0 \mathrm{c} 0$ & $\mathrm{NaN}$ & $\mathrm{NaN}$ & \\
\hline 759 & -------- & -- & ----- & ----- & ----- & -- & -- & -- & -- & B1V \\
\hline 760 & -------- & -- & ----- & ----- & ----- & -- & -- & -- & -- & \\
\hline 761 & -------- & -- & ----- & ----- & ----- & -- & -- & -- & -- & \\
\hline 762 & $10451002-5944378$ & 0.50 & $14.66 \pm 0.05$ & $13.68 \pm 0.04$ & $13.42 \pm 0.05$ & AAE & $\mathrm{c} 0 \mathrm{c}$ & $\mathrm{NaN}$ & 2.00 & \\
\hline 763 & $10451008-5943533$ & 0.53 & $14.64 \pm 0.04$ & $13.61 \pm 0.04$ & $13.32 \pm 0.07$ & AAA & $\mathrm{ccc}$ & 2.98 & 2.01 & \\
\hline 764 & -------- & -- & ----- & ----- & ----- & -- & -- & -- & -- & \\
\hline 765 & $10451026-5943072$ & 0.36 & $15.51 \pm * * * *$ & $13.98 \pm * * * *$ & $14.72 \pm 0.17$ & UUC & $00 \mathrm{c}$ & $\mathrm{NaN}$ & $\mathrm{NaN}$ & \\
\hline 766 & $10451022-5947290$ & 0.35 & $16.29 \pm * * * *$ & $14.79 \pm 0.08$ & $14.19 \pm 0.10$ & UAA & 000 & $\mathrm{NaN}$ & $\mathrm{NaN}$ & \\
\hline 767 & $10451030-5942298$ & 0.51 & $14.05 \pm * * * *$ & $13.54 \pm 0.06$ & $12.72 \pm * * * *$ & UAU & 0c0 & $\mathrm{NaN}$ & $\mathrm{NaN}$ & \\
\hline 768 & $10451033-5947060$ & 0.52 & $14.89 \pm 0.05$ & $13.61 \pm 0.05$ & $12.81 \pm 0.04$ & AAA & 000 & 11.34 & 1.84 & K-excess \\
\hline 769 & -------- & -- & ----- & ----- & ----- & -- & -- & -- & -- & \\
\hline 770 & -------- & -- & ----- & ----- & ----- & -- & -- & -- & -- & \\
\hline 771 & -------- & -- & ----- & ----- & ----- & -- & -- & -- & -- & \\
\hline 772 & $10451043-5944094$ & 0.34 & $15.40 \pm 0.08$ & $14.37 \pm 0.06$ & $14.12 \pm 0.08$ & AAA & $\mathrm{ccc}$ & 1.53 & 1.40 & \\
\hline 773 & $10451062-5945126$ & 0.57 & $11.56 \pm 0.02$ & $11.09 \pm 0.02$ & $10.67 \pm 0.02$ & AAA & 000 & $\mathrm{NaN}$ & 8.57 & K-excess \\
\hline 774 & -------- & -- & ----- & ----- & ----- & -- & -- & -- & -- & \\
\hline 775 & $10451065-5942543$ & 0.03 & $14.22 \pm 0.04$ & $13.51 \pm 0.05$ & $13.27 \pm 0.06$ & AAA & $\mathrm{ccc}$ & 2.14 & 2.22 & \\
\hline 776 & $10451068-5944233$ & 0.15 & $16.46 \pm 0.23$ & $15.10 \pm 0.09$ & $14.83 \pm 0.19$ & $\mathrm{DAC}$ & $\mathrm{ccc}$ & $\mathrm{NaN}$ & 0.72 & \\
\hline 777 & -------- & -- & ----- & ----- & ----- & -- & -- & -- & -- & \\
\hline 778 & $10451073-5937484$ & 0.56 & $14.80 \pm 0.08$ & $14.01 \pm 0.09$ & $12.85 \pm * * * *$ & $\mathrm{AAU}$ & $\operatorname{cc} 0$ & $\mathrm{NaN}$ & 1.91 & \\
\hline 779 & -------- & -- & ----- & ----- & ----- & -- & -- & -- & -- & \\
\hline 780 & -------- & -- & ----- & ----- & ----- & -- & -- & -- & -- & \\
\hline 781 & -------- & -- & ----- & ----- & ----- & -- & -- & -- & -- & \\
\hline
\end{tabular}


J. F. Albacete-Colombo et al.: An X-ray survey of low-mass stars in Trumpler 16 with Chandra, Online Material p 36

Table 2. continued.

\begin{tabular}{|c|c|c|c|c|c|c|c|c|c|c|}
\hline$N_{x}$ & 2MASS J+ & Off." & $J$ mag & $H$ mag & $K_{\mathrm{s}} \mathrm{mag}$ & Ph.Q & Cont. & $A_{\mathrm{v}}$ & Mass & ID.flags \\
\hline 782 & -------- & -- & ----- & ----- & ----- & -- & -- & -- & -- & \\
\hline 783 & -------- & -- & ----- & ----- & ----- & -- & -- & -- & -- & \\
\hline 784 & $10451101-5942392$ & 0.14 & $13.32 \pm * * * *$ & $13.06 \pm 0.04$ & $12.60 \pm 0.04$ & UAA & $0 \mathrm{cc}$ & $\mathrm{NaN}$ & $\mathrm{NaN}$ & \\
\hline 785 & $10451103-5945207$ & 0.16 & $15.63 \pm 0.09$ & $14.31 \pm * * * *$ & $13.97 \pm * * * *$ & AUU & $\mathrm{c} 00$ & $\mathrm{NaN}$ & 1.25 & \\
\hline 786 & $10451108-5944571$ & 0.52 & $13.68 \pm 0.10$ & $13.22 \pm 0.11$ & $13.00 \pm 0.08$ & $\mathrm{ABA}$ & $\mathrm{ccc}$ & $\mathrm{NaN}$ & 2.38 & \\
\hline 787 & $10451108-5945335$ & 0.13 & $13.89 \pm 0.02$ & $12.83 \pm 0.03$ & $12.19 \pm 0.02$ & AAA & 000 & 8.90 & 2.32 & K-excess \\
\hline 788 & $10451120-5942338$ & 0.58 & $11.93 \pm * * * *$ & $11.42 \pm 0.04$ & $11.02 \pm 0.04$ & UAA & $0 \mathrm{cc}$ & $\mathrm{NaN}$ & $\mathrm{NaN}$ & \\
\hline 789 & -------- & -- & ----- & ----- & ----- & -- & -- & -- & -- & \\
\hline 790 & -------- & -- & ----- & ----- & ----- & -- & -- & -- & -- & \\
\hline 791 & $10451134-5939033$ & 0.14 & $15.93 \pm 0.12$ & $14.98 \pm 0.10$ & $14.58 \pm 0.12$ & $\mathrm{BAB}$ & $\mathrm{ccc}$ & $\mathrm{NaN}$ & 1.07 & \\
\hline 792 & -------- & -- & ----- & ----- & ----- & -- & -- & -- & -- & \\
\hline 793 & $10451155-5946130$ & 0.38 & $15.31 \pm 0.08$ & $14.37 \pm 0.10$ & $13.85 \pm * * * *$ & AAU & $\operatorname{cc} 0$ & $\mathrm{NaN}$ & 1.46 & \\
\hline 794 & -------- & -- & ----- & ----- & ----- & -- & -- & -- & -- & \\
\hline 795 & -------- & -- & ----- & ----- & ----- & -- & -- & -- & -- & \\
\hline 796 & -------- & -- & ----- & ----- & ----- & -- & -- & -- & -- & \\
\hline 797 & -------- & -- & ----- & ----- & ----- & -- & -- & -- & -- & \\
\hline 798 & -------- & -- & ----- & ----- & ----- & -- & -- & -- & -- & \\
\hline 799 & -------- & -- & ----- & ----- & ----- & -- & -- & -- & -- & \\
\hline 800 & -------- & -- & ----- & ----- & ----- & -- & -- & -- & -- & \\
\hline 801 & -------- & -- & ----- & ----- & ----- & -- & -- & -- & -- & \\
\hline 802 & -------- & -- & ----- & ----- & ----- & -- & -- & -- & -- & \\
\hline 803 & $10451221-5945003$ & 0.11 & $8.68 \pm 0.01$ & $8.54 \pm 0.02$ & $8.43 \pm 0.02$ & AAA & $\mathrm{c} 00$ & 2.52 & 19.24 & O7V(n) \\
\hline 804 & -------- & -- & ----- & ----- & ----- & -- & -- & -- & -- & \\
\hline 805 & $10451248-5948452$ & 0.08 & $16.91 \pm * * * *$ & $15.37 \pm 0.12$ & $14.21 \pm 0.09$ & UBA & 000 & $\mathrm{NaN}$ & $\mathrm{NaN}$ & \\
\hline 806 & -------- & -- & ----- & ----- & ----- & -- & -- & -- & -- & \\
\hline 807 & $10451265-5942487$ & 0.37 & $9.83 \pm 0.02$ & $9.65 \pm 0.03$ & $9.53 \pm 0.02$ & AAA & $0 \mathrm{cc}$ & 2.62 & 14.99 & $\mathrm{~B} 2 \mathrm{~V}$ \\
\hline 808 & $10451271-5944460$ & 0.39 & $8.33 \pm 0.01$ & $8.18 \pm 0.03$ & $8.09 \pm 0.02$ & AAA & 000 & 2.21 & 20.54 & $09.5 \mathrm{~V}$ \\
\hline 809 & -------- & -- & ----- & ----- & ----- & -- & -- & -- & -- & \\
\hline 810 & -------- & -- & ----- & ----- & ----- & -- & -- & -- & -- & \\
\hline 811 & $10451287-5949421$ & 0.25 & $15.67 \pm 0.07$ & $13.97 \pm 0.05$ & $13.23 \pm 0.05$ & AAA & 000 & 10.34 & 1.22 & \\
\hline 812 & $10451287-5944192$ & 0.55 & $8.08 \pm 0.02$ & $7.89 \pm 0.03$ & $7.76 \pm 0.02$ & AAA & 000 & 2.98 & 21.48 & O9V \\
\hline 813 & -------- & -- & ----- & ----- & ----- & -- & -- & -- & -- & \\
\hline 814 & $10451283-5945040$ & 0.39 & $13.01 \pm 0.06$ & $12.03 \pm 0.21$ & $11.91 \pm 0.07$ & AEE & $\mathrm{ccc}$ & $\mathrm{NaN}$ & 3.55 & Mass-deg \\
\hline 815 & -------- & -- & ----- & ----- & ----- & -- & -- & -- & -- & \\
\hline 816 & $10451296-5935208$ & 0.32 & $13.73 \pm 0.06$ & $12.20 \pm * * * *$ & $11.85 \pm * * * *$ & AUU & $\mathrm{c} 00$ & $\mathrm{NaN}$ & 2.36 & \\
\hline 817 & -------- & -- & ----- & ----- & ----- & -- & -- & -- & -- & \\
\hline 818 & $10451326-5942581$ & 0.30 & $11.64 \pm * * * *$ & $11.50 \pm 0.07$ & $10.82 \pm * * * *$ & UAU & 0p0 & $\mathrm{NaN}$ & $\mathrm{NaN}$ & \\
\hline 819 & -------- & -- & ----- & ----- & ----- & -- & -- & -- & -- & \\
\hline 820 & $10451330-5943014$ & 0.88 & $11.90 \pm * * * *$ & $11.83 \pm 0.04$ & $10.81 \pm * * * *$ & UAU & 0p0 & $\mathrm{NaN}$ & $\mathrm{NaN}$ & \\
\hline 821 & $10451355-5943318$ & 1.09 & $12.53 \pm 0.03$ & $12.21 \pm 0.05$ & $11.99 \pm 0.06$ & AAA & $\mathrm{ccc}$ & 2.26 & 5.24 & \\
\hline 822 & $10451355-5944043$ & 1.36 & $13.84 \pm 0.06$ & $13.06 \pm 0.05$ & $12.70 \pm 0.06$ & AAA & $\mathrm{ccc}$ & 4.38 & 2.33 & \\
\hline 823 & $10451353-5951088$ & 0.78 & $14.32 \pm 0.04$ & $13.69 \pm 0.03$ & $13.43 \pm 0.04$ & AAA & 0ss & 2.41 & 2.19 & \\
\hline 824 & -------- & -- & ----- & ----- & ----- & -- & -- & -- & -- & \\
\hline 825 & $10451364-5944116$ & 1.39 & $14.53 \pm 0.22$ & $13.08 \pm * * * *$ & $12.86 \pm * * * *$ & DUU & $\mathrm{c} 00$ & $\mathrm{NaN}$ & 2.07 & \\
\hline 826 & -------- & -- & ----- & ----- & ----- & -- & -- & -- & -- & \\
\hline 827 & $10451380-5944463$ & 0.21 & $12.39 \pm * * * *$ & $12.66 \pm 0.04$ & $12.09 \pm 0.06$ & UAA & $0 \mathrm{cc}$ & $\mathrm{NaN}$ & $\mathrm{NaN}$ & \\
\hline 828 & $10451395-5943394$ & 0.14 & $14.98 \pm * * * *$ & $14.70 \pm 0.13$ & $14.18 \pm 0.11$ & UBB & $0 \mathrm{cc}$ & $\mathrm{NaN}$ & $\mathrm{NaN}$ & \\
\hline 829 & -------- & -- & ----- & ----- & ----- & -- & -- & -- & -- & \\
\hline 830 & $10451405-5943155$ & 0.57 & $14.58 \pm 0.04$ & $13.70 \pm 0.04$ & $13.49 \pm 0.05$ & AAE & $\operatorname{ccc}$ & $\mathrm{NaN}$ & 2.04 & \\
\hline 831 & $10451412-5942118$ & 0.44 & $14.90 \pm * * * *$ & $14.66 \pm 0.08$ & $13.60 \pm * * * *$ & UAU & $0 \mathrm{c} 0$ & $\mathrm{NaN}$ & $\mathrm{NaN}$ & \\
\hline 832 & -------- & -- & ----- & ----- & ----- & -- & -- & -- & -- & \\
\hline 833 & $10451414-5938582$ & 0.58 & $15.23 \pm 0.12$ & $14.22 \pm 0.09$ & $13.85 \pm 0.10$ & BAA & $\mathrm{ccc}$ & $\mathrm{NaN}$ & 1.53 & \\
\hline 834 & $10451452-5942470$ & 0.51 & $14.14 \pm 0.03$ & $13.15 \pm 0.04$ & $12.82 \pm 0.04$ & AAA & $\mathrm{ccc}$ & 3.71 & 2.25 & \\
\hline 835 & -------- & -- & ----- & ----- & ----- & -- & -- & -- & -- & \\
\hline 836 & $10451478-5944235$ & 0.25 & $15.26 \pm 0.09$ & $14.30 \pm 0.07$ & $13.88 \pm 0.07$ & AAA & $\mathrm{ccc}$ & 4.74 & 1.50 & \\
\hline 837 & -------- & -- & ----- & ----- & ----- & -- & -- & -- & -- & \\
\hline 838 & -------- & -- & ----- & ----- & ----- & -- & -- & -- & -- & \\
\hline 839 & $10451489-5944416$ & 0.03 & $14.91 \pm 0.06$ & $14.11 \pm 0.05$ & $13.81 \pm 0.06$ & AAA & $\mathrm{ccc}$ & 2.82 & 1.82 & \\
\hline 840 & $10451478-5944235$ & 0.48 & $15.26 \pm 0.09$ & $14.30 \pm 0.07$ & $13.88 \pm 0.07$ & AAA & $\mathrm{ccc}$ & 4.74 & 1.50 & \\
\hline 841 & $10451504-5940216$ & 0.31 & $15.08 \pm * * * *$ & $14.36 \pm 0.06$ & $13.79 \pm * * * *$ & UAU & $0 \mathrm{c} 0$ & $\mathrm{NaN}$ & $\mathrm{NaN}$ & \\
\hline 842 & ------- & -- & ----- & ----- & ----- & -- & -- & -- & -- & \\
\hline 843 & $10451526-5944166$ & 0.83 & $14.82 \pm 0.06$ & $14.05 \pm 0.06$ & $13.71 \pm 0.07$ & AAA & $\mathrm{ccc}$ & 3.72 & 1.89 & \\
\hline 844 & -------- & -- & ----- & ----- & ----- & -- & -- & -- & -- & \\
\hline 845 & $10451551-5940128$ & 0.23 & $13.92 \pm * * * *$ & $13.48 \pm 0.04$ & $13.06 \pm * * * *$ & UAU & 0c0 & $\mathrm{NaN}$ & $\mathrm{NaN}$ & \\
\hline 846 & $10451563-5943591$ & 1.34 & $15.82 \pm * * * *$ & $14.62 \pm * * * *$ & $15.01 \pm 0.21$ & UUC & $00 \mathrm{c}$ & $\mathrm{NaN}$ & $\mathrm{NaN}$ & \\
\hline
\end{tabular}


Table 2. continued.

\begin{tabular}{|c|c|c|c|c|c|c|c|c|c|c|}
\hline$N_{x}$ & $2 \mathrm{MASS} J+$ & Off." & $J \mathrm{mag}$ & $H$ mag & $K_{\mathrm{s}} \mathrm{mag}$ & Ph.Q & Cont. & $A_{\mathrm{v}}$ & Mass & ID.flags \\
\hline 847 & $10451578-5941271$ & 0.30 & $15.49 \pm * * * *$ & $14.30 \pm 0.05$ & $14.19 \pm * * * *$ & UAU & $0 \mathrm{c} 0$ & $\mathrm{NaN}$ & $\mathrm{NaN}$ & \\
\hline 848 & $10451622-5941411$ & 0.17 & $12.00 \pm * * * *$ & $11.56 \pm 0.02$ & $11.48 \pm * * * *$ & UAU & 000 & $\mathrm{NaN}$ & $\mathrm{NaN}$ & \\
\hline 849 & -------- & -- & ----- & ----- & ----- & -- & -- & -- & -- & \\
\hline 850 & -------- & -- & ----- & ----- & ----- & -- & -- & -- & -- & \\
\hline 851 & -------- & -- & ----- & ----- & ----- & -- & -- & -- & -- & \\
\hline 852 & -------- & -- & ----- & ----- & ----- & -- & -- & -- & -- & \\
\hline 853 & -------- & -- & ----- & ----- & ----- & -- & -- & -- & -- & \\
\hline 854 & $10451651-5943370$ & 0.12 & $8.15 \pm 0.00$ & $7.99 \pm 0.02$ & $7.88 \pm 0.03$ & AAA & 000 & 2.52 & 21.22 & $\mathrm{O} 5 \mathrm{~V}$ \\
\hline 855 & $10451645-5950167$ & 0.62 & $15.93 \pm * * * *$ & $14.98 \pm 0.11$ & $14.31 \pm 0.10$ & UBA & 000 & $\mathrm{NaN}$ & $\mathrm{NaN}$ & \\
\hline 856 & $10451665-5944203$ & 0.21 & $16.01 \pm * * * *$ & $15.21 \pm 0.11$ & $14.61 \pm 0.12$ & UBB & $0 \mathrm{cc}$ & $\mathrm{NaN}$ & $\mathrm{NaN}$ & \\
\hline 857 & $10451675-5940079$ & 0.21 & $14.20 \pm * * * *$ & $13.46 \pm 0.04$ & $12.82 \pm 0.05$ & UAA & $0 \mathrm{cc}$ & $\mathrm{NaN}$ & $\mathrm{NaN}$ & \\
\hline 858 & $10451684-5938087$ & 0.39 & $15.18 \pm 0.06$ & $14.44 \pm 0.05$ & $13.99 \pm 0.07$ & AAA & $\mathrm{c} 0 \mathrm{c}$ & 5.33 & 1.58 & \\
\hline 859 & $10451675-5946287$ & 2.09 & $12.96 \pm 0.02$ & $12.65 \pm 0.03$ & $12.63 \pm 0.03$ & AAA & 000 & $\mathrm{NaN}$ & 3.55 & Mass-deg. \\
\hline 860 & -------- & -- & ----- & ----- & ----- & -- & -- & -- & -- & \\
\hline 861 & $10451705-5945421$ & 0.66 & $15.16 \pm 0.06$ & $13.99 \pm 0.04$ & $13.59 \pm 0.05$ & AAA & $\operatorname{cc} 0$ & 4.55 & 1.59 & \\
\hline 862 & $10451717-5947013$ & 0.25 & $12.05 \pm 0.02$ & $11.10 \pm 0.02$ & $10.61 \pm 0.02$ & AAA & 000 & $\mathrm{NaN}$ & 6.76 & \\
\hline 863 & $10451722-5949132$ & 0.37 & $15.62 \pm * * * *$ & $15.19 \pm 0.11$ & $14.20 \pm 0.09$ & UBA & $0 \mathrm{cc}$ & $\mathrm{NaN}$ & $\mathrm{NaN}$ & \\
\hline 864 & -------- & -- & ----- & ----- & ----- & -- & -- & -- & -- & \\
\hline 865 & -------- & -- & ----- & ----- & ----- & -- & -- & -- & -- & \\
\hline 866 & -------- & -- & ----- & ----- & ----- & -- & -- & -- & -- & \\
\hline 867 & $10451762-5940543$ & 0.61 & $14.88 \pm * * * *$ & $14.28 \pm 0.07$ & $14.04 \pm 0.09$ & UAA & $0 \mathrm{cc}$ & $\mathrm{NaN}$ & $\mathrm{NaN}$ & \\
\hline 868 & $10451767-5938333$ & 0.35 & $14.83 \pm 0.05$ & $13.91 \pm 0.05$ & $13.64 \pm 0.05$ & AAA & $00 \mathrm{c}$ & 2.58 & 1.88 & \\
\hline 869 & -------- & -- & ----- & ----- & ----- & -- & -- & -- & -- & \\
\hline 870 & $10451776-5942284$ & 0.21 & $14.43 \pm * * * *$ & $13.40 \pm * * * *$ & $13.56 \pm 0.05$ & UUA & $00 \mathrm{c}$ & $\mathrm{NaN}$ & $\mathrm{NaN}$ & \\
\hline 871 & $10451788-5937095$ & 0.12 & $14.50 \pm 0.05$ & $13.63 \pm 0.05$ & $13.37 \pm 0.06$ & AAA & 000 & 2.37 & 2.09 & \\
\hline 872 & $10451799-5935591$ & 0.39 & $14.54 \pm 0.05$ & $13.59 \pm 0.05$ & $13.22 \pm 0.05$ & AAA & 000 & 4.31 & 2.07 & \\
\hline 873 & $10451810-5943059$ & 0.43 & $15.11 \pm 0.05$ & $14.11 \pm 0.04$ & $14.01 \pm 0.07$ & AAA & $\mathrm{c} 0 \mathrm{c}$ & $\mathrm{NaN}$ & 1.64 & \\
\hline 874 & -------- & -- & ----- & ----- & ----- & -- & -- & -- & -- & \\
\hline 875 & $10451837-5946133$ & 1.73 & $15.31 \pm 0.13$ & $14.01 \pm 0.06$ & $13.30 \pm 0.05$ & BAA & $\mathrm{ccc}$ & $\mathrm{NaN}$ & 1.46 & \\
\hline 876 & -------- & -- & ----- & ----- & ----- & -- & -- & -- & -- & \\
\hline 877 & $10451872-5939122$ & 0.62 & $15.74 \pm 0.09$ & $15.03 \pm 0.10$ & $14.72 \pm 0.12$ & $\mathrm{AAB}$ & $\mathrm{ccc}$ & $\mathrm{NaN}$ & 1.18 & \\
\hline 878 & -------- & -- & ----- & ----- & ----- & -- & -- & -- & -- & \\
\hline 879 & $10451894-5942184$ & 0.25 & $11.42 \pm 0.02$ & $11.24 \pm 0.03$ & $11.16 \pm 0.03$ & AAA & 000 & 1.45 & 9.09 & \\
\hline 880 & $10451899-5941427$ & 0.10 & $12.69 \pm 0.02$ & $12.35 \pm 0.03$ & $12.20 \pm 0.03$ & AAA & $\mathrm{ccc}$ & 0.89 & 4.86 & \\
\hline 881 & $10451912-5943594$ & 0.22 & $16.22 \pm 0.16$ & $15.20 \pm 0.16$ & $13.44 \pm * * * *$ & $\mathrm{CCU}$ & $\operatorname{cc} 0$ & $\mathrm{NaN}$ & 0.89 & \\
\hline 882 & $10451978-5947312$ & 0.28 & $16.16 \pm 0.12$ & $14.38 \pm 0.05$ & $13.31 \pm 0.04$ & BAA & 000 & $\mathrm{NaN}$ & 0.93 & \\
\hline 883 & $10451977-5949379$ & 0.54 & $16.17 \pm 0.13$ & $14.58 \pm 0.07$ & $13.82 \pm 0.06$ & BAA & 000 & $\mathrm{NaN}$ & 0.92 & \\
\hline 884 & $10451984-5943263$ & 0.53 & $15.36 \pm 0.07$ & $14.29 \pm 0.05$ & $13.92 \pm 0.09$ & AAA & $\mathrm{c} 0 \mathrm{c}$ & 4.00 & 1.42 & \\
\hline 885 & $10452009-5948467$ & 0.70 & $16.92 \pm * * * *$ & $14.81 \pm 0.07$ & $13.83 \pm 0.07$ & UAA & $0 \mathrm{c} 0$ & $\mathrm{NaN}$ & $\mathrm{NaN}$ & \\
\hline 886 & $10452014-5939196$ & 1.42 & $14.82 \pm 0.04$ & $13.99 \pm 0.03$ & $13.83 \pm 0.06$ & AAA & $00 \mathrm{c}$ & 0.48 & 1.89 & \\
\hline 887 & -------- & -- & ----- & ----- & ----- & -- & -- & -- & -- & \\
\hline 888 & $10452057-5942512$ & 0.27 & $9.39 \pm 0.02$ & $9.31 \pm 0.02$ & $9.26 \pm 0.02$ & AAA & 000 & 1.62 & 16.63 & $08.5 \mathrm{~V}$ \\
\hline 889 & -------- & -- & ----- & ----- & ----- & -- & -- & -- & -- & \\
\hline 890 & $10452067-5944014$ & 0.22 & $14.66 \pm 0.05$ & $13.76 \pm 0.04$ & $13.44 \pm 0.04$ & AAA & $\mathrm{ccc}$ & 3.33 & 2.00 & \\
\hline 891 & $10452078-5948078$ & 0.89 & $15.14 \pm 0.05$ & $13.16 \pm 0.03$ & $12.14 \pm 0.03$ & AAA & 000 & $\mathrm{NaN}$ & 1.62 & \\
\hline 892 & $10452088-5940130$ & 0.32 & $12.31 \pm 0.02$ & $11.86 \pm 0.03$ & $11.73 \pm 0.02$ & AAA & 000 & 0.70 & 5.84 & \\
\hline 893 & $10452092-5945301$ & 0.25 & $15.83 \pm 0.11$ & $14.80 \pm 0.08$ & $14.48 \pm 0.10$ & AAA & $\mathrm{ccc}$ & 0.13 & 1.13 & \\
\hline 894 & $10452096-5945488$ & 0.38 & $13.10 \pm 0.02$ & $12.14 \pm 0.03$ & $11.79 \pm 0.02$ & AAA & 000 & 4.23 & 3.55 & Mass-deg. \\
\hline 895 & $10452108-5944469$ & 0.44 & $16.30 \pm * * * *$ & $15.59 \pm 0.17$ & $14.65 \pm 0.14$ & UCB & $0 \mathrm{c} 0$ & $\mathrm{NaN}$ & $\mathrm{NaN}$ & \\
\hline 896 & $10452136-5941345$ & 0.27 & $14.35 \pm 0.04$ & $13.52 \pm 0.04$ & $13.32 \pm 0.05$ & AAA & $\mathrm{c} 0 \mathrm{c}$ & 1.50 & 2.17 & \\
\hline 897 & $10452142-5940471$ & 0.19 & $16.35 \pm 0.16$ & $15.23 \pm 0.11$ & $14.98 \pm 0.17$ & $\mathrm{BBC}$ & $\mathrm{ccc}$ & $\mathrm{NaN}$ & 0.81 & \\
\hline 898 & $10452140-5944203$ & 0.14 & $16.94 \pm * * * *$ & $16.41 \pm * * * *$ & $14.89 \pm 0.17$ & UUC & $00 \mathrm{c}$ & $\mathrm{NaN}$ & $\mathrm{NaN}$ & \\
\hline 899 & $10452137-5944312$ & 0.52 & $13.62 \pm * * * *$ & $12.52 \pm 0.05$ & $12.09 \pm 0.04$ & UAA & $0 \mathrm{cc}$ & $\mathrm{NaN}$ & $\mathrm{NaN}$ & \\
\hline 900 & -------- & -- & ----- & ----- & ----- & -- & -- & -- & -- & \\
\hline 901 & $10452178-5942054$ & 0.16 & $16.06 \pm 0.14$ & $15.00 \pm 0.10$ & $14.61 \pm 0.13$ & BAB & $\mathrm{ccc}$ & $\mathrm{NaN}$ & 0.99 & \\
\hline 902 & $10452195-5940214$ & 0.39 & $15.67 \pm 0.09$ & $14.80 \pm 0.08$ & $14.21 \pm 0.09$ & AAA & $\mathrm{ccc}$ & 7.53 & 1.22 & K-excess \\
\hline 903 & $10452200-5940527$ & 0.61 & $13.48 \pm 0.01$ & $12.62 \pm 0.02$ & $12.24 \pm 0.02$ & AAA & 000 & 4.73 & 2.44 & \\
\hline 904 & $10452199-5943563$ & 1.18 & $15.79 \pm * * * *$ & $15.04 \pm * * * *$ & $14.62 \pm 0.14$ & UUB & $00 \mathrm{c}$ & $\mathrm{NaN}$ & $\mathrm{NaN}$ & \\
\hline 905 & $10452206-5941458$ & 0.80 & $12.76 \pm 0.02$ & $12.14 \pm 0.03$ & $11.93 \pm 0.03$ & AAA & 000 & 2.01 & 4.71 & \\
\hline 906 & $10452214-5937554$ & 0.21 & $14.38 \pm 0.02$ & $13.62 \pm 0.03$ & $13.44 \pm 0.05$ & AAA & 000 & 1.04 & 2.16 & \\
\hline 907 & $10452211-5943361$ & 0.08 & $14.79 \pm 0.04$ & $13.97 \pm 0.04$ & $13.71 \pm 0.06$ & AAA & 000 & 2.20 & 1.91 & \\
\hline 908 & $10452209-5951147$ & 0.28 & $14.44 \pm * * * *$ & $13.83 \pm 0.07$ & $13.16 \pm * * * *$ & UAU & $0 \mathrm{c} 0$ & $\mathrm{NaN}$ & $\mathrm{NaN}$ & \\
\hline 909 & $10452227-5950470$ & 0.20 & $8.62 \pm 0.01$ & $8.19 \pm 0.01$ & $7.88 \pm 0.03$ & AAA & 000 & 5.62 & 19.47 & \\
\hline 910 & -------- & -- & ----- & ----- & ----- & -- & -- & -- & -- & \\
\hline 911 & $10452308-5945023$ & 0.17 & $16.46 \pm 0.18$ & $15.33 \pm 0.16$ & $15.16 \pm * * * *$ & $\mathrm{CCU}$ & $\operatorname{cc} 0$ & $\mathrm{NaN}$ & 0.72 & \\
\hline 912 & -------- & -- & ----- & ----- & ----- & -- & -- & -- & -- & \\
\hline 913 & $10452355-5939141$ & 0.21 & $13.24 \pm 0.02$ & $12.96 \pm 0.03$ & $12.81 \pm 0.03$ & AAA & 000 & 0.80 & 3.55 & Mass-deg. \\
\hline
\end{tabular}


Table 2. continued.

\begin{tabular}{|c|c|c|c|c|c|c|c|c|c|c|}
\hline$\overline{N_{x}}$ & 2 MASS J+ & Off." & $J \mathrm{mag}$ & $H \mathrm{mag}$ & $K_{\mathrm{s}} \mathrm{mag}$ & Ph.Q & Cont. & $A_{\mathrm{v}}$ & Mass & ID.flags \\
\hline 914 & $10452355-5945204$ & 0.92 & $15.88 \pm 0.10$ & $14.90 \pm 0.07$ & $14.48 \pm 0.10$ & $\overline{\mathrm{AAA}}$ & $\operatorname{css}$ & 2.21 & 1.10 & \\
\hline 915 & $10452374-5945468$ & 0.37 & $14.64 \pm 0.05$ & $13.29 \pm 0.05$ & $12.66 \pm 0.04$ & AAA & 000 & 8.78 & 2.01 & \\
\hline 916 & $10452370-5941446$ & 0.19 & $14.56 \pm 0.03$ & $13.72 \pm 0.04$ & $13.45 \pm 0.04$ & AAE & $00 \mathrm{c}$ & $\mathrm{NaN}$ & 2.06 & \\
\hline 917 & $10452382-5945018$ & 0.13 & $15.73 \pm 0.09$ & $13.99 \pm * * * *$ & $14.02 \pm * * * *$ & AUU & $\mathrm{c} 00$ & $\mathrm{NaN}$ & 1.19 & \\
\hline 918 & -------- & -- & ----- & ----- & ----- & -- & -- & -- & -- & \\
\hline 919 & $10452408-5944123$ & 0.15 & $16.28 \pm 0.22$ & $15.24 \pm 0.11$ & $14.80 \pm * * * *$ & CAU & $\operatorname{cc} 0$ & $\mathrm{NaN}$ & 0.85 & \\
\hline 920 & -------- & -- & ----- & ----- & ----- & -- & -- & -- & -- & \\
\hline 921 & $10452405-5947514$ & 1.52 & $14.73 \pm 0.06$ & $13.94 \pm 0.06$ & $13.47 \pm 0.06$ & AAA & $\mathrm{c} 0 \mathrm{c}$ & 6.00 & 1.95 & K-excess \\
\hline 922 & $10452448-5942207$ & 0.27 & $15.01 \pm 0.06$ & $14.08 \pm 0.05$ & $13.42 \pm * * * *$ & AAU & $\operatorname{cc} 0$ & $\mathrm{NaN}$ & 1.73 & \\
\hline 923 & $10452462-5945066$ & 0.56 & $14.09 \pm 0.02$ & $13.16 \pm 0.02$ & $12.57 \pm 0.02$ & AAA & 000 & 8.05 & 2.26 & K-excess \\
\hline 924 & $10452483-5941260$ & 0.79 & $13.98 \pm 0.04$ & $13.26 \pm 0.04$ & $13.03 \pm 0.05$ & AAA & 000 & 2.08 & 2.29 & \\
\hline 925 & -------- & -- & ----- & ----- & ----- & -- & -- & -- & -- & \\
\hline 926 & $10452531-5941258$ & 1.11 & $14.34 \pm * * * *$ & $15.04 \pm 0.11$ & $13.40 \pm * * * *$ & UBU & 0p0 & $\mathrm{NaN}$ & $\mathrm{NaN}$ & \\
\hline 927 & -------- & -- & ----- & ----- & ----- & -- & -- & -- & -- & \\
\hline 928 & $10452557-5943508$ & 0.28 & $13.37 \pm 0.04$ & $12.63 \pm 0.04$ & $12.32 \pm 0.04$ & AAA & $\mathrm{ccc}$ & 3.54 & 2.47 & \\
\hline 929 & $10452560-5949347$ & 0.25 & $17.88 \pm * * * *$ & $15.46 \pm 0.12$ & $14.07 \pm 0.09$ & UBA & 000 & $\mathrm{NaN}$ & $\mathrm{NaN}$ & \\
\hline 930 & -------- & -- & ----- & ----- & ----- & -- & -- & -- & -- & \\
\hline 931 & $10452561-5942319$ & 1.76 & $13.38 \pm 0.06$ & $13.08 \pm 0.05$ & $12.88 \pm 0.10$ & AAA & $\mathrm{ccc}$ & 1.57 & 2.46 & \\
\hline 932 & $10452569-5947538$ & 0.20 & $16.94 \pm * * * *$ & $15.59 \pm 0.14$ & $14.50 \pm 0.10$ & UBA & 000 & $\mathrm{NaN}$ & $\mathrm{NaN}$ & \\
\hline 933 & $10452592-5946560$ & 0.27 & $16.71 \pm * * * *$ & $15.46 \pm 0.13$ & $14.55 \pm 0.16$ & UBC & $0 \mathrm{cc}$ & $\mathrm{NaN}$ & $\mathrm{NaN}$ & \\
\hline 934 & $10452592-5944042$ & 0.27 & $15.37 \pm 0.09$ & $14.37 \pm 0.06$ & $14.05 \pm 0.09$ & AAA & $\mathrm{ccc}$ & 3.08 & 1.42 & \\
\hline 935 & $10452648-5940305$ & 0.81 & $14.49 \pm * * * *$ & $13.82 \pm 0.07$ & $13.20 \pm 0.06$ & UAA & $0 \mathrm{cc}$ & $\mathrm{NaN}$ & $\mathrm{NaN}$ & \\
\hline 936 & -------- & -- & ----- & ----- & ----- & -- & -- & -- & -- & \\
\hline 937 & $10452680-5948450$ & 0.57 & $15.19 \pm * * * *$ & $15.12 \pm 0.11$ & $14.01 \pm 0.08$ & UAA & $0 \mathrm{cc}$ & $\mathrm{NaN}$ & $\mathrm{NaN}$ & \\
\hline 938 & $10452689-5948285$ & 0.21 & $17.83 \pm * * * *$ & $16.69 \pm * * * *$ & $14.15 \pm 0.09$ & UUA & 000 & $\mathrm{NaN}$ & $\mathrm{NaN}$ & \\
\hline 939 & $10452694-5941495$ & 0.28 & $15.28 \pm 0.06$ & $14.35 \pm 0.05$ & $13.97 \pm 0.08$ & AAA & $00 \mathrm{c}$ & 4.28 & 1.48 & \\
\hline 940 & $10452702-5942317$ & 1.51 & $14.86 \pm 0.05$ & $13.96 \pm 0.06$ & $13.43 \pm 0.06$ & AAA & $0 \mathrm{c} 0$ & 6.96 & 1.86 & K-excess \\
\hline 941 & $10452683-5938576$ & 0.93 & $15.73 \pm 0.09$ & $14.17 \pm * * * *$ & $13.91 \pm * * * *$ & AUU & $\mathrm{c} 00$ & $\mathrm{NaN}$ & 1.19 & \\
\hline 942 & -------- & -- & ----- & ----- & ----- & -- & -- & -- & -- & \\
\hline 943 & $10452732-5945187$ & 0.35 & $13.16 \pm 0.03$ & $12.39 \pm 0.03$ & $11.94 \pm 0.03$ & AAA & sss & 5.93 & 3.55 & Mass-deg. \\
\hline 944 & $10452722-5942194$ & 0.34 & $15.36 \pm 0.07$ & $14.40 \pm 0.05$ & $14.10 \pm 0.07$ & AAA & $0 \mathrm{cc}$ & 2.63 & 1.42 & \\
\hline 945 & $10452782-5942339$ & 0.62 & $14.39 \pm * * * *$ & $15.44 \pm 0.15$ & $13.69 \pm * * * *$ & UBU & $0 \mathrm{c} 0$ & $\mathrm{NaN}$ & $\mathrm{NaN}$ & \\
\hline 946 & $10452795-5941389$ & 1.64 & $13.05 \pm 0.02$ & $12.36 \pm 0.02$ & $12.18 \pm 0.03$ & AAA & 000 & 1.62 & 3.55 & Mass-deg. \\
\hline 947 & -------- & -- & ----- & ----- & ----- & -- & -- & -- & -- & \\
\hline 948 & $10452825-5942304$ & 1.69 & $13.43 \pm 0.02$ & $13.00 \pm 0.03$ & $12.81 \pm 0.04$ & AAA & 000 & 1.55 & 2.45 & \\
\hline 949 & -------- & -- & ----- & ----- & ----- & -- & -- & -- & -- & \\
\hline 950 & $10452818-5941036$ & 0.20 & $15.12 \pm * * * *$ & $15.03 \pm 0.12$ & $13.75 \pm * * * *$ & UBU & $0 \mathrm{c} 0$ & $\mathrm{NaN}$ & $\mathrm{NaN}$ & \\
\hline 951 & $10452822-5947080$ & 1.60 & $15.32 \pm 0.06$ & $13.77 \pm 0.05$ & $13.03 \pm 0.04$ & AAA & 000 & 10.47 & 1.45 & \\
\hline 952 & $10452825-5945564$ & 0.02 & $13.24 \pm 0.02$ & $12.42 \pm 0.03$ & $11.98 \pm 0.03$ & AAA & 000 & 5.77 & 3.55 & Mass-deg. \\
\hline 953 & $10452829-5940230$ & 0.16 & $14.52 \pm * * * *$ & $13.64 \pm * * * *$ & $13.75 \pm 0.08$ & UUA & $00 \mathrm{c}$ & $\mathrm{NaN}$ & $\mathrm{NaN}$ & \\
\hline 954 & $10452846-5941555$ & 0.71 & $12.83 \pm 0.02$ & $12.49 \pm 0.02$ & $12.40 \pm 0.03$ & AAA & 000 & 0.07 & 4.59 & \\
\hline 955 & $10452849-5942469$ & 0.66 & $13.81 \pm 0.04$ & $13.01 \pm 0.04$ & $12.51 \pm 0.04$ & AAA & 000 & 6.54 & 2.34 & K-excess \\
\hline 956 & -------- & -- & ----- & ----- & ----- & -- & -- & -- & -- & \\
\hline 957 & $10452862-5947553$ & 0.53 & $11.91 \pm 0.02$ & $10.27 \pm 0.02$ & $9.46 \pm 0.02$ & AAA & 000 & 13.70 & 7.27 & \\
\hline 958 & $10452887-5943478$ & 0.66 & $13.79 \pm 0.04$ & $12.75 \pm 0.04$ & $12.34 \pm 0.04$ & AEA & 000 & $\mathrm{NaN}$ & 2.35 & \\
\hline 959 & $10452945-5946498$ & 0.42 & $17.60 \pm * * * *$ & $15.27 \pm 0.17$ & $14.26 \pm 0.12$ & UCB & $0 \mathrm{cc}$ & $\mathrm{NaN}$ & $\mathrm{NaN}$ & \\
\hline 960 & -------- & -- & ----- & ----- & ----- & -- & -- & -- & -- & \\
\hline 961 & $10453024-5948206$ & 0.64 & $10.43 \pm 0.02$ & $9.24 \pm 0.03$ & $8.60 \pm 0.02$ & AAA & $00 \mathrm{~d}$ & 10.97 & 12.75 & \\
\hline 962 & -------- & -- & ----- & ----- & ----- & -- & -- & -- & -- & \\
\hline 963 & $10453037-5947527$ & 0.37 & $17.28 \pm * * * *$ & $15.32 \pm 0.12$ & $14.34 \pm 0.10$ & UBA & $0 \mathrm{cc}$ & $\mathrm{NaN}$ & $\mathrm{NaN}$ & \\
\hline 964 & $10453070-5940349$ & 0.23 & $15.40 \pm 0.07$ & $14.52 \pm 0.06$ & $14.31 \pm 0.12$ & AAB & $00 \mathrm{c}$ & $\mathrm{NaN}$ & 1.40 & \\
\hline 965 & -------- & -- & ----- & ----- & ----- & -- & -- & -- & -- & \\
\hline 966 & $10453095-5950451$ & 0.13 & $12.59 \pm 0.07$ & $11.32 \pm 0.05$ & $10.39 \pm 0.04$ & AAA & $\operatorname{ccc}$ & 15.63 & 5.07 & K-excess \\
\hline 967 & $10453134-5941133$ & 0.22 & $11.53 \pm 0.02$ & $11.34 \pm 0.02$ & $11.25 \pm 0.02$ & AAA & 000 & 0.13 & 8.68 & \\
\hline 968 & $10453202-5942384$ & 0.57 & $12.86 \pm * * * *$ & $14.63 \pm 0.18$ & $14.29 \pm 0.09$ & UCA & $0 \mathrm{cc}$ & $\mathrm{NaN}$ & $\mathrm{NaN}$ & \\
\hline 969 & $10453208-5947507$ & 0.37 & $14.83 \pm 0.05$ & $14.26 \pm 0.07$ & $13.72 \pm * * * *$ & AAU & $\operatorname{cc} 0$ & $\mathrm{NaN}$ & 1.88 & \\
\hline 970 & $10453244-5948197$ & 0.65 & $14.80 \pm 0.07$ & $13.55 \pm * * * *$ & $12.96 \pm * * * *$ & AUU & 000 & $\mathrm{NaN}$ & 1.91 & \\
\hline 971 & $10453227-5943196$ & 0.25 & $14.20 \pm 0.03$ & $13.25 \pm 0.04$ & $12.89 \pm 0.04$ & AAA & 000 & 4.32 & 2.23 & \\
\hline 972 & $10453260-5946345$ & 0.53 & $15.78 \pm 0.09$ & $14.23 \pm 0.04$ & $13.51 \pm 0.04$ & AAA & 000 & 10.07 & 1.16 & \\
\hline 973 & $10453263-5948572$ & 0.49 & $15.22 \pm 0.06$ & $13.86 \pm 0.05$ & $13.25 \pm 0.05$ & AAA & 000 & 8.38 & 1.54 & \\
\hline 974 & $10453270-5949167$ & 1.67 & $15.76 \pm 0.10$ & $14.24 \pm 0.04$ & $13.34 \pm 0.03$ & AAA & 000 & 13.00 & 1.17 & $\mathrm{~K}$-excess \\
\hline 975 & $10453277-5947469$ & 0.25 & $16.83 \pm * * * *$ & $14.54 \pm 0.06$ & $13.39 \pm 0.06$ & UAA & 000 & $\mathrm{NaN}$ & $\mathrm{NaN}$ & \\
\hline 976 & $10453302-5941335$ & 0.48 & $15.63 \pm 0.08$ & $14.76 \pm 0.04$ & $14.39 \pm 0.10$ & AAA & $0 \mathrm{cc}$ & 1.35 & 1.25 & \\
\hline 977 & -------- & -- & ----- & ----- & ----- & -- & -- & -- & -- & \\
\hline 978 & $10453339-5949071$ & 0.36 & $14.64 \pm * * * *$ & $13.58 \pm 0.07$ & $12.27 \pm * * * *$ & UAU & $0 \mathrm{c} 0$ & $\mathrm{NaN}$ & $\mathrm{NaN}$ & \\
\hline 979 & $10453367-5947147$ & 0.14 & $11.66 \pm 0.02$ & $11.58 \pm 0.03$ & $11.54 \pm 0.03$ & AAA & 000 & 1.64 & 8.22 & \\
\hline 980 & $10453370-5948083$ & 0.30 & $15.88 \pm * * * *$ & $14.68 \pm * * * *$ & $14.64 \pm 0.12$ & UUB & $00 \mathrm{c}$ & $\mathrm{NaN}$ & $\mathrm{NaN}$ & \\
\hline
\end{tabular}


Table 2. continued.

\begin{tabular}{|c|c|c|c|c|c|c|c|c|c|c|}
\hline$\overline{\bar{N}}$ & 2MASS J+ & $\overline{\text { Off." }}$ & $J$ mag & $H$ mag & $K_{\mathrm{s}} \mathrm{mag}$ & $\overline{P h . Q}$ & Cont. & $\overline{A_{\mathrm{v}}}$ & Mass & $\overline{\text { ID.flags }}$ \\
\hline 981 & $10453391-5950091$ & 0.88 & $15.08 \pm 0.05$ & $13.81 \pm 0.04$ & $13.12 \pm 0.04$ & $\overline{\mathrm{AAA}}$ & $\mathrm{c} 00$ & 9.62 & 1.67 & \\
\hline 982 & $10453417-5950411$ & 0.86 & $14.66 \pm 0.06$ & $13.64 \pm 0.04$ & $13.25 \pm 0.05$ & AAA & $\mathrm{ccc}$ & 4.73 & 2.00 & \\
\hline 983 & -------- & -- & ----- & ----- & ----- & -- & -- & -- & -- & \\
\hline 984 & $10453445-5949157$ & 0.59 & $15.45 \pm 0.06$ & $14.34 \pm 0.05$ & $13.82 \pm 0.06$ & AAA & cpp & 6.56 & 1.36 & \\
\hline 985 & $10453484-5946281$ & 0.19 & $16.62 \pm 0.16$ & $14.57 \pm 0.05$ & $13.65 \pm 0.06$ & CAA & 000 & $\mathrm{NaN}$ & 0.66 & \\
\hline 986 & -------- & -- & ----- & ----- & ----- & -- & -- & -- & -- & \\
\hline 987 & $10453541-5945447$ & 1.04 & $13.26 \pm 0.03$ & $12.47 \pm 0.03$ & $11.95 \pm 0.03$ & AAA & 000 & 6.94 & 2.50 & K-excess \\
\hline 988 & -------- & -- & ----- & ----- & ----- & -- & -- & -- & -- & \\
\hline 989 & -------- & -- & ----- & ----- & ----- & -- & -- & -- & -- & \\
\hline 990 & $10453592-5941136$ & 0.27 & $15.09 \pm 0.06$ & $14.30 \pm 0.07$ & $14.12 \pm 0.07$ & AAA & $0 \mathrm{cc}$ & 0.10 & 1.66 & \\
\hline 991 & $10453643-5947563$ & 0.51 & $15.72 \pm * * * *$ & $14.92 \pm 0.10$ & $14.01 \pm 0.11$ & UAA & $0 \mathrm{cc}$ & $\mathrm{NaN}$ & $\mathrm{NaN}$ & \\
\hline 992 & $10453635-5948379$ & 0.42 & $15.39 \pm 0.08$ & $14.11 \pm 0.04$ & $13.53 \pm 0.05$ & AAA & $\mathrm{ccc}$ & 7.68 & 1.41 & \\
\hline 993 & $10453660-5944110$ & 0.71 & $9.83 \pm 0.02$ & $9.19 \pm 0.03$ & $8.77 \pm 0.02$ & AAA & $0 \mathrm{~d} 0$ & 7.40 & 14.98 & K-excess \\
\hline 994 & -------- & -- & ----- & ----- & ----- & -- & -- & -- & -- & \\
\hline 995 & $10453669-5948085$ & 0.20 & $14.46 \pm * * * *$ & $14.85 \pm 0.07$ & $12.82 \pm * * * *$ & UAU & 0c0 & $\mathrm{NaN}$ & $\mathrm{NaN}$ & \\
\hline 996 & $10453674-5947020$ & 0.61 & $9.76 \pm 0.02$ & $8.88 \pm 0.03$ & $8.33 \pm 0.02$ & AAA & 000 & 9.46 & 15.26 & K-excess \\
\hline 997 & $10453699-5948203$ & 0.79 & $13.60 \pm 0.14$ & $12.71 \pm 0.11$ & $12.13 \pm 0.09$ & BBA & $\mathrm{cpc}$ & $\mathrm{NaN}$ & 2.40 & \\
\hline 998 & $10453685-5946202$ & 1.02 & $15.45 \pm 0.05$ & $13.78 \pm 0.03$ & $13.02 \pm 0.03$ & AAA & 000 & 10.66 & 1.37 & \\
\hline 999 & $10453723-5946463$ & 0.63 & $16.35 \pm * * * *$ & $15.61 \pm 0.13$ & $14.25 \pm 0.10$ & UBA & $0 \mathrm{cc}$ & $\mathrm{NaN}$ & $\mathrm{NaN}$ & \\
\hline 1000 & $10453774-5947552$ & 0.41 & $13.13 \pm 0.04$ & $12.17 \pm 0.03$ & $11.63 \pm 0.04$ & AAA & 000 & 7.81 & 3.55 & Mass-deg. \\
\hline 1001 & $10453825-5950293$ & 1.87 & $14.31 \pm 0.13$ & $13.27 \pm 0.28$ & $12.86 \pm 0.16$ & $\mathrm{BDC}$ & $\mathrm{ccc}$ & $\mathrm{NaN}$ & 2.20 & \\
\hline 1002 & $10453834-5942078$ & 0.15 & $11.36 \pm 0.03$ & $10.80 \pm 0.03$ & $10.24 \pm 0.02$ & AAA & 000 & 9.62 & 9.30 & K-excess \\
\hline 1003 & $10453860-5945133$ & 0.38 & $12.70 \pm 0.02$ & $11.76 \pm 0.03$ & $11.12 \pm 0.02$ & AAA & 000 & $\mathrm{NaN}$ & 4.84 & K-excess \\
\hline 1004 & $10453926-5947395$ & 1.02 & $15.44 \pm 0.08$ & $13.45 \pm 0.06$ & $12.47 \pm 0.06$ & AAA & 000 & 14.59 & 1.38 & \\
\hline 1005 & $10453914-5945271$ & 0.37 & $15.06 \pm 0.06$ & $13.85 \pm 0.07$ & $13.30 \pm 0.05$ & AAA & 000 & 7.35 & 1.69 & \\
\hline 1006 & $10454000-5948594$ & 0.87 & $14.52 \pm 0.04$ & $13.13 \pm 0.05$ & $12.36 \pm 0.04$ & AAA & 000 & 10.95 & 2.07 & K-excess \\
\hline 1007 & $10454012-5949587$ & 0.36 & $16.21 \pm 0.15$ & $14.87 \pm 0.10$ & $14.50 \pm 0.11$ & BAB & 000 & $\mathrm{NaN}$ & 0.90 & \\
\hline 1008 & -------- & -- & ----- & ----- & ----- & -- & -- & -- & -- & \\
\hline 1009 & $10454029-5948297$ & 1.09 & $11.81 \pm 0.02$ & $11.04 \pm 0.03$ & $10.52 \pm 0.02$ & AAA & 000 & 9.10 & 7.66 & K-excess \\
\hline 1010 & $10454009-5949255$ & 0.55 & $16.03 \pm 0.12$ & $14.83 \pm 0.10$ & $14.30 \pm 0.09$ & BAA & $\mathrm{c} 00$ & $\mathrm{NaN}$ & 1.00 & \\
\hline 1011 & $10454039-5938374$ & 1.02 & $13.46 \pm 0.02$ & $13.11 \pm 0.03$ & $12.88 \pm 0.03$ & AAA & 000 & 2.24 & 2.44 & \\
\hline 1012 & $10454054-5947424$ & 0.59 & $16.50 \pm 0.16$ & $14.44 \pm 0.06$ & $13.30 \pm 0.04$ & BAA & 000 & $\mathrm{NaN}$ & 0.69 & \\
\hline 1013 & $10454048-5947521$ & 0.10 & $15.93 \pm 0.10$ & $14.15 \pm 0.05$ & $13.18 \pm 0.05$ & AAA & 000 & 14.19 & 1.07 & K-excess \\
\hline 1014 & $10454160-5938201$ & 1.84 & $12.69 \pm 0.02$ & $12.26 \pm 0.04$ & $12.13 \pm 0.03$ & AAA & 000 & 0.61 & 4.85 & \\
\hline 1015 & $10454158-5948240$ & 0.14 & $14.15 \pm 0.04$ & $12.89 \pm * * * *$ & $12.41 \pm * * * *$ & AUU & $\mathrm{c} 00$ & $\mathrm{NaN}$ & 2.25 & \\
\hline 1016 & -------- & -- & ----- & ----- & ----- & -- & -- & -- & -- & \\
\hline 1017 & $10454236-5942539$ & 0.18 & $14.38 \pm 0.03$ & $13.30 \pm 0.04$ & $12.79 \pm 0.03$ & AAA & 000 & 6.84 & 2.16 & \\
\hline 1018 & $10454256-5940222$ & 0.99 & $15.22 \pm 0.06$ & $14.41 \pm 0.07$ & $14.04 \pm 0.07$ & AAA & 000 & 3.86 & 1.53 & \\
\hline 1019 & $10454365-5939540$ & 0.36 & $11.93 \pm 0.02$ & $11.76 \pm 0.02$ & $11.65 \pm 0.02$ & AAA & 000 & 0.49 & 7.20 & \\
\hline 1020 & $10454366-5941481$ & 0.80 & $13.39 \pm 0.03$ & $12.39 \pm 0.04$ & $11.96 \pm 0.03$ & AAA & 000 & 5.51 & 2.46 & \\
\hline 1021 & -------- & -- & ----- & ---- & ----- & -- & -- & -- & -- & \\
\hline 1022 & -------- & -- & ----- & ----- & ----- & -- & -- & -- & -- & \\
\hline 1023 & $10454449-5939258$ & 1.12 & $12.74 \pm 0.02$ & $12.42 \pm 0.02$ & $12.23 \pm 0.03$ & AAA & 000 & 1.74 & 4.76 & \\
\hline 1024 & $10454505-5941552$ & 0.39 & $14.73 \pm 0.06$ & $13.66 \pm 0.05$ & $13.22 \pm 0.05$ & AAA & 000 & 5.52 & 1.95 & \\
\hline 1025 & $10454557-5949481$ & 0.22 & $15.02 \pm 0.07$ & $13.97 \pm 0.06$ & $13.50 \pm 0.06$ & AAA & $\mathrm{ccc}$ & 5.75 & 1.73 & \\
\hline 1026 & $10454572-5945176$ & 0.40 & $17.81 \pm * * * *$ & $16.28 \pm * * * *$ & $14.88 \pm 0.14$ & UUB & 000 & $\mathrm{NaN}$ & $\mathrm{NaN}$ & \\
\hline 1027 & $10454607-5946384$ & 1.28 & $12.95 \pm 0.02$ & $12.77 \pm 0.02$ & $12.69 \pm 0.03$ & AAA & 000 & $\mathrm{NaN}$ & 3.55 & Mass-deg. \\
\hline 1028 & -------- & -- & ----- & ----- & ----- & -- & -- & -- & -- & \\
\hline 1029 & $10454661-5948404$ & 0.21 & $11.59 \pm 0.02$ & $10.94 \pm 0.03$ & $10.56 \pm 0.02$ & AAA & 000 & $\mathrm{NaN}$ & 8.45 & \\
\hline 1030 & $10454707-5950415$ & 0.14 & $15.59 \pm 0.09$ & $14.52 \pm 0.05$ & $14.10 \pm 0.07$ & AAA & $\mathrm{ccc}$ & 4.65 & 1.28 & \\
\hline 1031 & $10454785-5947488$ & 0.37 & $17.81 \pm * * * *$ & $15.87 \pm 0.15$ & $14.65 \pm 0.11$ & UCB & 000 & $\mathrm{NaN}$ & $\mathrm{NaN}$ & \\
\hline 1032 & -------- & -- & ----- & ----- & ----- & -- & -- & -- & -- & \\
\hline 1033 & $10455025-5947039$ & 0.96 & $15.17 \pm * * * *$ & $14.62 \pm 0.09$ & $13.76 \pm 0.06$ & UAA & 000 & $\mathrm{NaN}$ & $\mathrm{NaN}$ & \\
\hline 1034 & $10455163-5945348$ & 0.07 & $15.75 \pm * * * *$ & $14.82 \pm * * * *$ & $14.33 \pm 0.10$ & UUA & 000 & $\mathrm{NaN}$ & $\mathrm{NaN}$ & \\
\hline 1035 & $10455226-5948102$ & 1.56 & $15.28 \pm 0.08$ & $14.11 \pm 0.06$ & $13.68 \pm 0.08$ & AAA & $\mathrm{ccc}$ & 5.00 & 1.48 & \\
\hline
\end{tabular}


Table 3. X-ray source spectroscopy.

\begin{tabular}{|c|c|c|c|c|c|c|}
\hline $\begin{array}{l}N_{x} \\
\#\end{array}$ & $\begin{array}{l}\text { Cnts. } \\
\text { (ph) }\end{array}$ & $\begin{array}{l}\text { Stat. } \\
\left(\chi_{v}^{2}\right)\end{array}$ & $\begin{array}{l}\log \left(N_{\mathrm{H}}\right) \\
\left(\mathrm{cm}^{-2}\right)\end{array}$ & $\begin{array}{l}k T \\
(\mathrm{keV})\end{array}$ & $\begin{array}{l}\log \left(L_{\mathrm{x}}\right) \\
(\mathrm{erg} / \mathrm{s})\end{array}$ & flag \\
\hline 1 & 30 & -- & ----- & ----- & 30.51 & no-fit \\
\hline 2 & 36 & -- & ----- & ----- & 30.58 & no-fit \\
\hline 3 & 208 & 0.66 & $22.01 \pm 0.16$ & $2.07 \pm 0.56$ & 31.58 & fitted \\
\hline 4 & 55 & 0.94 & $21.90 \pm 0.31$ & $2.96 \pm 2.61$ & 30.50 & fitted \\
\hline 5 & 28 & -- & ----- & ----- & 30.48 & no-fit \\
\hline 6 & 41 & -- & ----- & ----- & 30.64 & no-fit \\
\hline 7 & 47 & -- & ----- & ----- & 31.29 & Tr16-19 \\
\hline 8 & 54 & -- & ----- & ----- & 30.76 & no-fit \\
\hline 9 & 45 & -- & ----- & ----- & 30.68 & no-fit \\
\hline 10 & 25 & -- & ----- & ----- & 30.42 & no-fit \\
\hline 11 & 41 & -- & ----- & ----- & 30.72 & no-fit \\
\hline 12 & 132 & 1.50 & $21.23 \pm 0.62$ & $2.65 \pm 0.94$ & 31.08 & fitted \\
\hline 13 & 89 & 0.40 & $21.64 \pm 0.14$ & $0.67 \pm 0.93$ & 30.83 & fitted \\
\hline 14 & 15 & -- & ----- & ----- & 30.23 & no-fit \\
\hline 15 & 41 & -- & ----- & ----- & 30.65 & no-fit \\
\hline 16 & 23 & -- & ----- & ----- & 30.40 & no-fit \\
\hline 17 & 28 & -- & ----- & ----- & 30.48 & no-fit \\
\hline 18 & 31 & 0.80 & $22.32 \pm 0.32$ & $1.36 \pm 0.88$ & 31.04 & fitted \\
\hline 19 & 7 & -- & ----- & ----- & 29.93 & no-fit \\
\hline 20 & 30 & -- & ----- & ----- & 30.51 & no-fit \\
\hline 21 & 25 & 1.21 & $21.97 \pm 0.65$ & $0.29 \pm 0.18$ & 30.02 & fitted \\
\hline 22 & 176 & 0.94 & $21.81 \pm 0.22$ & $3.32 \pm 1.45$ & 31.46 & fitted \\
\hline 23 & 25 & -- & ----- & ----- & 30.43 & no-fit \\
\hline 24 & 22 & -- & ----- & ----- & 30.38 & no-fit \\
\hline 25 & 177 & 1.87 & $22.00 \pm 0.18$ & $2.17 \pm 0.74$ & 31.52 & fitted \\
\hline 26 & 44 & 0.90 & $21.70 \pm 0.27$ & $1.49 \pm 0.54$ & 30.38 & fitted \\
\hline 27 & 51 & 0.50 & $22.06 \pm 0.32$ & $2.11 \pm 1.27$ & 31.03 & fitted \\
\hline 28 & 77 & 0.47 & $21.28 \pm 0.72$ & $1.49 \pm 0.44$ & 30.83 & fitted \\
\hline 29 & 36 & -- & ----- & ----- & 30.59 & no-fit \\
\hline 30 & 12 & -- & ----- & ----- & 30.12 & no-fit \\
\hline 31 & 37 & -- & ----- & ----- & 30.60 & no-fit \\
\hline 32 & 16 & -- & ----- & ----- & 30.25 & no-fit \\
\hline 33 & 40 & 0.52 & $22.30 \pm 0.19$ & $0.74 \pm 0.28$ & 31.65 & fitted \\
\hline 34 & 42 & -- & ----- & ----- & 30.64 & no-fit \\
\hline 35 & 63 & -- & ----- & ----- & 30.83 & no-fit \\
\hline 36 & 416 & 0.55 & $22.13 \pm 0.08$ & $7.85 \pm 3.72$ & 31.93 & fitted \\
\hline 37 & 58 & -- & ----- & ----- & 30.79 & no-fit \\
\hline 38 & 38 & 1.06 & $21.53 \pm 1.00$ & $64.00 \pm * * * *$ & 30.55 & hard tail \\
\hline 39 & 34 & 0.68 & $21.74 \pm 0.37$ & $3.65 \pm 4.96$ & 30.36 & fitted \\
\hline 40 & 11 & -- & ----- & ----- & 30.07 & no-fit \\
\hline 41 & 218 & 1.61 & $21.22 \pm 2.37$ & $2.84 \pm 1.01$ & 31.18 & fitted \\
\hline 42 & 37 & 0.76 & $21.63 \pm 0.49$ & $47.11 \pm * * * *$ & 30.63 & hard tail \\
\hline 43 & 688 & 1.52 & $21.09 \pm 1.59$ & $1.88 \pm 0.16$ & 31.71 & fitted \\
\hline 44 & 9 & -- & ----- & ----- & 29.98 & no-fit \\
\hline 45 & 28 & 0.98 & $22.41 \pm 0.93$ & $64.00 \pm * * * *$ & 31.62 & hard tail \\
\hline 46 & 89 & 0.72 & $21.36 \pm 0.65$ & $3.35 \pm 1.90$ & 31.02 & fitted \\
\hline 47 & 18 & -- & ----- & ----- & 30.39 & no-fit \\
\hline 48 & 19 & -- & ----- & ----- & 30.31 & no-fit \\
\hline 49 & 34 & -- & ----- & ----- & 30.56 & no-fit \\
\hline 50 & 27 & 0.58 & $21.73 \pm 0.61$ & $0.73 \pm 0.23$ & 29.97 & fitted \\
\hline 51 & 13 & -- & ----- & ----- & 30.15 & no-fit \\
\hline 52 & 156 & 0.90 & $21.41 \pm 0.35$ & $1.59 \pm 0.28$ & 31.18 & $\operatorname{Tr} 16-124$ \\
\hline 53 & 16 & -- & ----- & ----- & 30.23 & no-fit \\
\hline 54 & 11 & -- & ----- & ----- & 30.10 & no-fit \\
\hline 55 & 12 & -- & ----- & ----- & 30.10 & no-fit \\
\hline 56 & 129 & 0.64 & $21.30 \pm 0.46$ & $2.44 \pm 0.86$ & 31.05 & fitted \\
\hline 57 & 27 & 0.53 & $22.27 \pm 0.36$ & $1.20 \pm 0.78$ & 30.89 & fitted \\
\hline 58 & 69 & 1.39 & $21.59 \pm 0.32$ & $0.75 \pm 0.21$ & 30.86 & fitted \\
\hline 59 & 3 & -- & ----- & ----- & 29.00 & no-fit \\
\hline 60 & 66 & 0.57 & $22.35 \pm 0.26$ & $7.65 \pm * * * *$ & 31.35 & fitted \\
\hline 61 & 40 & 0.90 & $22.22 \pm 0.32$ & $1.68 \pm 1.09$ & 30.94 & fitted \\
\hline 62 & 47 & 0.88 & $22.01 \pm 0.28$ & $1.09 \pm 0.53$ & 30.96 & fitted \\
\hline 63 & 31 & -- & ----- & ----- & 30.61 & no-fit \\
\hline 64 & 33 & -- & ----- & ----- & 30.55 & no-fit \\
\hline 65 & 52 & 0.78 & $22.17 \pm 0.15$ & $0.86 \pm 0.32$ & 33.72 & fitted \\
\hline
\end{tabular}

Table 3. continued.

\begin{tabular}{|c|c|c|c|c|c|c|}
\hline $\begin{array}{l}N_{x} \\
\#\end{array}$ & $\begin{array}{l}\text { Cnts. } \\
(\mathrm{ph})\end{array}$ & $\begin{array}{l}\text { Stat. } \\
\left(\chi_{v}^{2}\right)\end{array}$ & $\begin{array}{l}\log \left(N_{\mathrm{H}}\right) \\
\left(\mathrm{cm}^{-2}\right)\end{array}$ & $\begin{array}{l}k T \\
(\mathrm{keV})\end{array}$ & $\begin{array}{l}\log \left(L_{\mathrm{X}}\right) \\
(\mathrm{erg} / \mathrm{s})\end{array}$ & flag \\
\hline 66 & 83 & 1.13 & $21.18 \pm 0.57$ & $2.63 \pm 1.20$ & 30.73 & fitted \\
\hline 67 & 15 & -- & ----- & ----- & 30.23 & no-fit \\
\hline 68 & 21 & 0.84 & $21.51 \pm 0.52$ & $1.65 \pm 0.86$ & 30.11 & fitted \\
\hline 69 & 37 & 0.94 & $22.03 \pm 0.27$ & $1.08 \pm 0.50$ & 30.93 & fitted \\
\hline 70 & 191 & 1.64 & $21.55 \pm 0.25$ & $1.80 \pm 0.45$ & 31.31 & fitted \\
\hline 71 & 377 & 0.98 & $21.47 \pm 0.68$ & $2.23 \pm 0.38$ & 31.52 & fitted \\
\hline 72 & 15 & -- & ----- & ----- & 30.20 & no-fit \\
\hline 73 & 22 & 0.80 & $21.50 \pm 0.84$ & $3.70 \pm 5.51$ & 30.28 & fitted \\
\hline 74 & 22476 & 12.73 & $21.69 \pm 0.14$ & $1.96 \pm 0.34$ & 33.52 & HD93162AB \\
\hline 75 & 59 & -- & ----- & ----- & 30.80 & no-fit \\
\hline 76 & 22 & 0.81 & $21.90 \pm 0.82$ & $1.62 \pm 1.65$ & 30.59 & fitted \\
\hline 77 & 4 & -- & ----- & ----- & 33.00 & fitted \\
\hline 78 & 9 & -- & ----- & ----- & 30.02 & no-fit \\
\hline 79 & 56 & 1.42 & $21.85 \pm 0.57$ & $1.66 \pm 1.01$ & 30.88 & fitted \\
\hline 80 & 9 & -- & ----- & ----- & 30.01 & no-fit \\
\hline 81 & 17 & -- & ----- & ----- & 30.25 & no-fit \\
\hline 82 & 44 & 0.88 & $22.02 \pm 0.16$ & $0.64 \pm 0.28$ & 31.20 & fitted \\
\hline 83 & 17 & -- & ----- & ----- & 30.27 & no-fit \\
\hline 84 & 22 & 0.80 & $21.83 \pm 0.80$ & $24.32 \pm * * * *$ & 30.39 & hard tail \\
\hline 85 & 25 & 0.39 & $21.82 \pm 0.18$ & $3.69 \pm 5.15$ & 30.23 & fitted \\
\hline 86 & 51 & 0.81 & $22.02 \pm 0.29$ & $1.25 \pm 0.49$ & 30.89 & fitted \\
\hline 87 & 184 & 0.75 & $21.43 \pm 0.25$ & $34.46 \pm * * * *$ & 31.34 & hard tail \\
\hline 88 & 17 & -- & ----- & ----- & 30.26 & no-fit \\
\hline 89 & 689 & 0.82 & $22.13 \pm 0.25$ & $0.59 \pm 0.36$ & 32.82 & Tr16-244 \\
\hline 90 & 12 & -- & ----- & ----- & 30.13 & no-fit \\
\hline 91 & 9 & -- & ----- & ----- & 30.01 & no-fit \\
\hline 92 & 49 & 0.71 & $21.51 \pm 0.39$ & $1.84 \pm 0.91$ & 30.57 & fitted \\
\hline 93 & 40 & 1.07 & $21.47 \pm 0.69$ & $0.35 \pm 0.22$ & 30.66 & fitted \\
\hline 94 & 3 & -- & ----- & ----- & 29.40 & no-fit \\
\hline 95 & 15 & -- & ----- & ----- & 30.23 & no-fit \\
\hline 96 & 12 & -- & ----- & ----- & 30.12 & no-fit \\
\hline 97 & 34 & 1.07 & $22.41 \pm 0.29$ & $1.38 \pm 0.67$ & 31.04 & fitted \\
\hline 98 & 26 & 0.47 & $21.41 \pm 0.64$ & $1.78 \pm 1.34$ & 30.34 & fitted \\
\hline 99 & 11 & -- & ----- & ----- & 30.09 & no-fit \\
\hline 100 & 26 & -- & ----- & ----- & 30.45 & no-fit \\
\hline 101 & 125 & 0.86 & $21.75 \pm 0.28$ & $1.60 \pm 0.43$ & 31.12 & fitted \\
\hline 102 & 25 & 0.34 & $21.11 \pm 2.13$ & $27.96 \pm * * * *$ & 30.38 & hard tail \\
\hline 103 & 52 & 0.45 & $22.45 \pm 0.22$ & $19.64 \pm * * * *$ & 31.15 & hard tail \\
\hline 104 & 51 & -- & ----- & ----- & 30.74 & no-fit \\
\hline 105 & 164 & 0.66 & $21.55 \pm 0.17$ & $2.49 \pm 0.56$ & 31.18 & fitted \\
\hline 106 & 15 & -- & ----- & ----- & 30.22 & no-fit \\
\hline 107 & 14 & -- & ----- & ----- & 30.19 & no-fit \\
\hline 108 & 7 & -- & ----- & ----- & 29.99 & no-fit \\
\hline 109 & 10 & -- & ----- & ----- & 30.04 & no-fit \\
\hline 110 & 195 & 3.20 & $21.90 \pm 0.16$ & $1.25 \pm 0.23$ & 31.46 & fitted \\
\hline 111 & 19 & -- & ----- & ----- & 30.32 & no-fit \\
\hline 112 & 74 & 0.89 & $21.28 \pm 0.48$ & $6.50 \pm 5.46$ & 30.85 & fitted \\
\hline 113 & 13 & -- & ----- & ----- & 30.17 & no-fit \\
\hline 114 & 23 & 0.39 & $23.02 \pm 0.53$ & $34.42 \pm * * * *$ & 31.11 & hard tail \\
\hline 115 & 34 & 0.65 & $21.77 \pm 0.58$ & $1.24 \pm 0.61$ & 31.61 & fitted \\
\hline 116 & 35 & 1.24 & $22.43 \pm 0.28$ & $1.20 \pm 0.77$ & 31.42 & fitted \\
\hline 117 & 18 & -- & ----- & ----- & 30.48 & no-fit \\
\hline 118 & 11 & -- & ----- & ----- & 30.10 & no-fit \\
\hline 119 & 65 & 0.43 & $21.62 \pm 0.34$ & $1.31 \pm 0.27$ & 30.79 & fitted \\
\hline 120 & 15 & -- & ----- & ----- & 30.23 & no-fit \\
\hline 121 & 37 & 0.49 & $21.53 \pm 0.48$ & $1.73 \pm 1.15$ & 30.58 & fitted \\
\hline 122 & 26 & 0.88 & $21.82 \pm 0.83$ & $1.37 \pm 1.08$ & 30.32 & fitted \\
\hline 123 & 13 & -- & ----- & ----- & 30.15 & no-fit \\
\hline 124 & 32 & -- & ----- & ----- & 30.53 & no-fit \\
\hline 125 & 25 & 0.79 & $21.63 \pm 0.72$ & $3.93 \pm 6.84$ & 30.35 & fitted \\
\hline 126 & 65 & 0.83 & $21.49 \pm 0.37$ & $1.37 \pm 0.37$ & 30.68 & fitted \\
\hline 127 & 7 & -- & ----- & ----- & 29.90 & no-fit \\
\hline 128 & 41 & 0.88 & $22.50 \pm 0.38$ & $64.00 \pm * * * *$ & 31.00 & hard tail \\
\hline 129 & 24 & 0.72 & $21.93 \pm 0.39$ & $1.04 \pm 0.57$ & 30.47 & fitted \\
\hline 130 & 19 & -- & ----- & ----- & 30.32 & no-fit \\
\hline
\end{tabular}


Table 3. continued.

\begin{tabular}{|c|c|c|c|c|c|c|}
\hline $\begin{array}{l}N_{x} \\
\#\end{array}$ & $\begin{array}{l}\text { Cnts. } \\
\text { (ph) }\end{array}$ & $\begin{array}{l}\text { Stat. } \\
\left(\chi_{y}^{2}\right)\end{array}$ & $\begin{array}{l}\log \left(N_{\mathrm{H}}\right) \\
\left(\mathrm{cm}^{-2}\right)\end{array}$ & $\begin{array}{l}k T \\
(\mathrm{keV})\end{array}$ & $\begin{array}{l}\log \left(L_{\mathrm{x}}\right) \\
(\mathrm{erg} / \mathrm{s})\end{array}$ & flag \\
\hline 131 & 7 & -- & ----- & ----- & 29.89 & no-fit \\
\hline 132 & 15 & -- & ----- & ----- & 30.21 & no-fit \\
\hline 133 & 118 & 0.70 & $21.25 \pm 0.38$ & $3.58 \pm 1.72$ & 30.98 & fitted \\
\hline 134 & 24 & 1.07 & $22.65 \pm 0.26$ & $0.76 \pm 0.46$ & 31.70 & fitted \\
\hline 135 & 9 & -- & ----- & ----- & 30.02 & no-fit \\
\hline 136 & 119 & 1.71 & $21.28 \pm 2.14$ & $3.05 \pm 1.48$ & 30.87 & $\operatorname{Tr} 16-11$ \\
\hline 137 & 4 & -- & ----- & ----- & 29.85 & no-fit \\
\hline 138 & 23 & 0.84 & $22.79 \pm 0.43$ & $0.27 \pm 0.17$ & 33.21 & fitted \\
\hline 139 & 5 & -- & ----- & ----- & 29.75 & no-fit \\
\hline 140 & 6 & -- & ----- & ----- & 30.01 & no-fit \\
\hline 141 & 51 & 1.06 & $21.70 \pm 0.41$ & $1.08 \pm 0.42$ & 30.70 & fitted \\
\hline 142 & 33 & 0.39 & $21.59 \pm 0.78$ & $2.73 \pm 2.64$ & 30.50 & fitted \\
\hline 143 & 18 & -- & ----- & ----- & 30.30 & no-fit \\
\hline 144 & 13 & -- & ----- & ----- & 30.15 & no-fit \\
\hline 145 & 31 & 0.66 & $21.99 \pm 0.21$ & $0.69 \pm 0.33$ & 30.86 & fitted \\
\hline 146 & 42 & 0.55 & $21.92 \pm 0.30$ & $1.09 \pm 0.45$ & 30.82 & fitted \\
\hline 147 & 88 & 0.54 & $21.83 \pm 0.64$ & $1.92 \pm 0.45$ & 30.72 & fitted \\
\hline 148 & 9 & -- & ----- & ----- & 30.01 & no-fit \\
\hline 149 & 17 & -- & ----- & ----- & 30.26 & no-fit \\
\hline 150 & 38 & 0.62 & $20.22 \pm 4.58$ & $3.31 \pm 2.21$ & 30.38 & fitted \\
\hline 151 & 159 & 1.05 & $21.50 \pm 0.25$ & $1.72 \pm 0.34$ & 31.09 & fitted \\
\hline 152 & 47 & 0.65 & $21.59 \pm 0.58$ & $6.12 \pm 8.28$ & 30.82 & fitted \\
\hline 153 & 58 & 0.67 & $21.76 \pm 0.19$ & $0.47 \pm 0.20$ & 31.10 & fitted \\
\hline 154 & 31 & -- & ----- & ----- & 30.53 & no-fit \\
\hline 155 & 23 & 0.67 & $22.10 \pm 0.33$ & $0.93 \pm 0.51$ & 30.73 & fitted \\
\hline 156 & 318 & 1.12 & $21.45 \pm 1.76$ & $2.60 \pm 0.54$ & 31.49 & fitted \\
\hline 157 & 34 & 0.34 & $21.67 \pm 0.51$ & $1.29 \pm 0.30$ & 30.27 & fitted \\
\hline 158 & 67 & 0.40 & $21.04 \pm 0.86$ & $2.05 \pm 0.70$ & 30.73 & fitted \\
\hline 159 & 22 & 0.81 & $21.26 \pm 1.23$ & $1.33 \pm 0.31$ & 29.92 & fitted \\
\hline 160 & 32 & 1.14 & $22.16 \pm 0.63$ & $1.68 \pm 1.88$ & 30.67 & fitted \\
\hline 161 & 7 & -- & ----- & ----- & 30.27 & no-fit \\
\hline 162 & 8 & -- & ----- & ----- & 29.98 & no-fit \\
\hline 163 & 19 & -- & ----- & ----- & 30.32 & no-fit \\
\hline 164 & 70 & 0.60 & $21.39 \pm 0.36$ & $2.23 \pm 1.30$ & 30.73 & fitted \\
\hline 165 & 21 & 0.74 & $22.05 \pm 0.35$ & $0.71 \pm 0.51$ & 30.73 & fitted \\
\hline 166 & 16 & -- & ----- & ----- & 30.25 & no-fit \\
\hline 167 & 6 & -- & ----- & ----- & 29.80 & no-fit \\
\hline 168 & 7 & -- & ----- & ----- & 29.87 & no-fit \\
\hline 169 & 30 & 0.66 & $21.72 \pm 0.83$ & $1.37 \pm 0.98$ & 30.53 & fitted \\
\hline 170 & 70 & 0.82 & $21.52 \pm 0.30$ & $2.32 \pm 1.23$ & 30.91 & fitted \\
\hline 171 & 8 & -- & ----- & ----- & 29.96 & no-fit \\
\hline 172 & 54 & 0.55 & $21.61 \pm 0.26$ & $33.15 \pm * * * *$ & 30.81 & hard tail \\
\hline 173 & 10 & -- & ----- & ----- & 30.05 & no-fit \\
\hline 174 & 129 & 1.25 & $21.46 \pm 0.22$ & $1.88 \pm 0.42$ & 31.09 & fitted \\
\hline 175 & 31 & 0.49 & $21.13 \pm 0.83$ & $1.58 \pm 0.46$ & 31.27 & fitted \\
\hline 176 & 33 & -- & ----- & ----- & 30.55 & no-fit \\
\hline 177 & 9 & -- & ----- & ----- & 30.00 & no-fit \\
\hline 178 & 12 & -- & ---- & ----- & 30.14 & no-fit \\
\hline 179 & 111 & 0.46 & $21.19 \pm 0.36$ & $0.57 \pm 0.76$ & 30.96 & fitted \\
\hline 180 & 55 & 0.60 & $21.76 \pm 0.17$ & $2.56 \pm 1.50$ & 30.62 & fitted \\
\hline 181 & 73 & 1.07 & $21.32 \pm 0.39$ & $2.76 \pm 1.84$ & 30.72 & fitted \\
\hline 182 & 37 & 0.49 & $21.34 \pm 0.54$ & $1.44 \pm 0.50$ & 30.40 & fitted \\
\hline 183 & 31 & 1.06 & $22.17 \pm 0.41$ & $1.57 \pm 1.40$ & 30.84 & fitted \\
\hline 184 & 64 & 1.60 & $22.06 \pm 0.12$ & $0.76 \pm 0.31$ & 31.24 & fitted \\
\hline 185 & 14 & -- & ----- & ----- & 30.20 & no-fit \\
\hline 186 & 6 & -- & ----- & ----- & 29.81 & no-fit \\
\hline 187 & 12 & -- & ----- & ----- & 30.12 & no-fit \\
\hline 188 & 30 & 0.61 & $21.80 \pm 0.13$ & $2.20 \pm 1.52$ & 30.11 & fitted \\
\hline 189 & 96 & 0.79 & $22.01 \pm 0.23$ & $1.46 \pm 0.54$ & 31.22 & fitted \\
\hline 190 & 9 & -- & ----- & ----- & 30.02 & no-fit \\
\hline 191 & 112 & 0.46 & $21.33 \pm 0.27$ & $2.30 \pm 0.77$ & 31.79 & fitted \\
\hline 192 & 29 & 0.48 & $21.47 \pm 0.50$ & $1.70 \pm 0.52$ & 30.43 & fitted \\
\hline 193 & 3 & -- & ----- & ----- & 29.51 & no-fit \\
\hline 194 & 3 & -- & ----- & ----- & 29.55 & no-fit \\
\hline 195 & 19 & -- & ----- & ----- & 30.32 & no-fit \\
\hline
\end{tabular}

Table 3. continued.

\begin{tabular}{|c|c|c|c|c|c|c|}
\hline $\begin{array}{l}N_{x} \\
\#\end{array}$ & $\begin{array}{l}\text { Cnts. } \\
\text { (ph) }\end{array}$ & $\begin{array}{l}\text { Stat. } \\
\left(\chi_{\nu}^{2}\right)\end{array}$ & $\begin{array}{l}\log \left(N_{\mathrm{H}}\right) \\
\left(\mathrm{cm}^{-2}\right)\end{array}$ & $\begin{array}{l}k T \\
(\mathrm{keV})\end{array}$ & $\begin{array}{l}\log \left(L_{\mathrm{x}}\right) \\
\text { (erg/s) }\end{array}$ & flag \\
\hline 196 & 205 & 0.91 & $21.88 \pm 0.15$ & $2.37 \pm 0.62$ & 31.46 & fitted \\
\hline 197 & 32 & 0.55 & $21.05 \pm 0.95$ & $1.45 \pm 0.47$ & 30.20 & fitted \\
\hline 198 & 44 & 0.67 & $21.76 \pm 0.39$ & $1.22 \pm 0.39$ & 30.63 & fitted \\
\hline 199 & 17 & -- & ----- & ----- & 30.27 & no-fit \\
\hline 200 & 55 & 0.60 & $21.83 \pm 0.31$ & $1.39 \pm 0.53$ & 30.96 & fitted \\
\hline 201 & 3 & -- & ----- & ----- & 29.55 & no-fit \\
\hline 202 & 56 & 1.14 & $21.23 \pm 1.57$ & $64.00 \pm * * * *$ & 30.70 & hard tail \\
\hline 203 & 12 & -- & ----- & ----- & 30.14 & no-fit \\
\hline 204 & 23 & 0.62 & $21.77 \pm 0.66$ & $2.16 \pm 1.94$ & 30.34 & fitted \\
\hline 205 & 71 & 0.86 & $21.61 \pm 0.15$ & $3.54 \pm 2.65$ & 33.28 & fitted \\
\hline 206 & 22 & 0.52 & $21.86 \pm 0.55$ & $1.10 \pm 0.72$ & 30.39 & fitted \\
\hline 207 & 149 & 1.35 & $21.92 \pm 0.50$ & $2.27 \pm 0.59$ & 31.00 & $\operatorname{Tr} 16-10$ \\
\hline 208 & 10 & -- & ----- & ----- & 30.05 & no-fit \\
\hline 209 & 18 & -- & ----- & ----- & 30.28 & no-fit \\
\hline 210 & 15 & -- & ----- & ----- & 30.20 & no-fit \\
\hline 211 & 41 & 0.27 & $22.06 \pm 0.35$ & $1.21 \pm 0.63$ & 31.19 & fitted \\
\hline 212 & 5 & -- & ----- & ----- & 29.75 & no-fit \\
\hline 213 & 102 & 0.64 & $21.24 \pm 0.35$ & $4.31 \pm 2.10$ & 30.95 & fitted \\
\hline 214 & 16 & -- & ----- & ----- & 30.24 & no-fit \\
\hline 215 & 5 & -- & ----- & ----- & 29.96 & no-fit \\
\hline 216 & 60 & 0.54 & $21.29 \pm 0.52$ & $7.25 \pm * * * *$ & 30.77 & fitted \\
\hline 217 & 18 & -- & ----- & ----- & 30.29 & no-fit \\
\hline 218 & 14 & -- & ----- & ----- & 30.18 & no-fit \\
\hline 219 & 3 & -- & ----- & ---- & 29.22 & no-fit \\
\hline 220 & 22 & 0.54 & $21.43 \pm 0.73$ & $3.03 \pm 3.27$ & 30.21 & fitted \\
\hline 221 & 45 & 0.75 & $21.72 \pm 0.43$ & $1.63 \pm 0.73$ & 30.60 & fitted \\
\hline 222 & 28 & 0.29 & $21.60 \pm 0.51$ & $1.66 \pm 0.49$ & 30.14 & fitted \\
\hline 223 & 84 & 1.23 & $21.17 \pm 0.51$ & $3.17 \pm 1.79$ & 30.79 & fitted \\
\hline 224 & 10 & -- & ----- & ----- & 30.05 & no-fit \\
\hline 225 & 20 & 0.69 & $21.97 \pm 0.43$ & $1.09 \pm 0.76$ & 30.64 & fitted \\
\hline 226 & 7 & -- & ----- & ----- & 29.88 & no-fit \\
\hline 227 & 18 & -- & ----- & ----- & 30.29 & no-fit \\
\hline 228 & 598 & 1.16 & $21.05 \pm 2.05$ & $0.60 \pm 0.35$ & 31.64 & HD93204 \\
\hline 229 & 48 & 1.00 & $21.28 \pm 0.49$ & $1.76 \pm 0.74$ & 30.42 & fitted \\
\hline 230 & 9 & -- & ----- & ----- & 30.01 & no-fit \\
\hline 231 & 39 & 1.07 & $21.93 \pm 0.19$ & $0.73 \pm 0.29$ & 30.83 & fitted \\
\hline 232 & 8 & -- & ----- & ----- & 29.97 & no-fit \\
\hline 233 & 17 & -- & ----- & ----- & 30.27 & no-fit \\
\hline 234 & 113 & 1.76 & $22.09 \pm 0.11$ & $0.86 \pm 0.26$ & 31.58 & fitted \\
\hline 235 & 33 & 0.61 & $21.26 \pm 0.51$ & $3.76 \pm 3.72$ & 30.30 & fitted \\
\hline 236 & 25 & 0.74 & $22.03 \pm 0.62$ & $1.48 \pm 2.14$ & 30.56 & fitted \\
\hline 237 & 80 & 1.15 & $21.42 \pm 0.40$ & $5.93 \pm 6.03$ & 30.86 & fitted \\
\hline 238 & 30 & 0.72 & $21.54 \pm 0.60$ & $0.93 \pm 0.33$ & 30.30 & fitted \\
\hline 239 & 25 & 0.43 & $21.97 \pm 0.62$ & $21.64 \pm * * * *$ & 31.47 & hard tail \\
\hline 240 & 56 & 1.26 & $21.96 \pm 0.43$ & $1.37 \pm 0.76$ & 30.91 & fitted \\
\hline 241 & 22 & 0.99 & $21.10 \pm 1.49$ & $63.99 \pm * * * *$ & 30.65 & hard tail \\
\hline 242 & 3351 & 2.71 & $21.55 \pm 0.32$ & $0.57 \pm 0.14$ & 32.58 & HD93205B \\
\hline 243 & 17 & -- & ----- & ----- & 30.27 & no-fit \\
\hline 244 & 17 & -- & ----- & ----- & 30.26 & no-fit \\
\hline 245 & 119 & 1.05 & $21.51 \pm 0.32$ & $2.61 \pm 0.98$ & 31.03 & fitted \\
\hline 246 & 15 & -- & ----- & ----- & 30.21 & no-fit \\
\hline 247 & 10 & -- & ---- & ----- & 30.04 & no-fit \\
\hline 248 & 27 & 0.58 & $22.03 \pm 0.37$ & $1.79 \pm 1.28$ & 30.63 & fitted \\
\hline 249 & 79 & 0.51 & $21.68 \pm 0.25$ & $4.63 \pm 4.52$ & 30.95 & fitted \\
\hline 250 & 9 & -- & ----- & ----- & 29.98 & no-fit \\
\hline 251 & 81 & 1.18 & $21.94 \pm 0.24$ & $1.26 \pm 0.39$ & 31.02 & fitted \\
\hline 252 & 6 & -- & ----- & ----- & 29.84 & no-fit \\
\hline 253 & 10 & -- & ----- & ----- & 30.04 & no-fit \\
\hline 254 & 120 & 1.07 & $21.29 \pm 0.30$ & $4.49 \pm 2.46$ & 31.00 & fitted \\
\hline 255 & 8 & -- & ----- & ----- & 29.96 & no-fit \\
\hline 256 & 140 & 0.91 & $21.68 \pm 0.25$ & $2.16 \pm 0.65$ & 31.32 & fitted \\
\hline 257 & 7 & -- & ----- & ----- & 29.91 & no-fit \\
\hline 258 & 12 & -- & ---- & ----- & 30.13 & no-fit \\
\hline 259 & 19 & -- & ----- & ----- & 30.32 & no-fit \\
\hline 260 & 56 & 0.73 & $21.60 \pm 0.30$ & $1.75 \pm 0.66$ & 30.61 & fitted \\
\hline
\end{tabular}


Table 3. continued.

\begin{tabular}{|c|c|c|c|c|c|c|}
\hline $\begin{array}{l}N_{x} \\
\#\end{array}$ & $\begin{array}{l}\text { Cnts. } \\
\text { (ph) }\end{array}$ & $\begin{array}{l}\text { Stat. } \\
\left(\chi_{v}^{2}\right)\end{array}$ & $\begin{array}{l}\log \left(N_{\mathrm{H}}\right) \\
\left(\mathrm{cm}^{-2}\right)\end{array}$ & $\begin{array}{l}k T \\
(\mathrm{keV})\end{array}$ & $\begin{array}{l}\log \left(L_{\mathrm{x}}\right) \\
(\mathrm{erg} / \mathrm{s})\end{array}$ & flag \\
\hline 261 & 7 & -- & ----- & ----- & 30.10 & no-fit \\
\hline 262 & 7 & -- & ----- & ----- & 30.10 & no-fit \\
\hline 263 & 19 & -- & ----- & ----- & 30.31 & no-fit \\
\hline 264 & 8 & -- & ----- & ----- & 29.97 & no-fit \\
\hline 265 & 18 & -- & ----- & ----- & 30.28 & no-fit \\
\hline 266 & 58 & 0.60 & $21.52 \pm 0.38$ & $1.20 \pm 0.26$ & 30.63 & fitted \\
\hline 267 & 11 & -- & ----- & ----- & 30.29 & no-fit \\
\hline 268 & 16 & -- & ----- & ----- & 30.24 & no-fit \\
\hline 269 & 5 & -- & ----- & ----- & 29.97 & no-fit \\
\hline 270 & 24 & -- & ----- & ----- & 30.41 & no-fit \\
\hline 271 & 324 & 0.58 & $21.70 \pm 0.11$ & $2.03 \pm 0.30$ & 31.52 & fitted \\
\hline 272 & 27 & 0.56 & $21.51 \pm 0.30$ & $2.87 \pm 2.78$ & 30.19 & fitted \\
\hline 273 & 56 & 0.70 & $21.30 \pm 0.62$ & $30.23 \pm * * * *$ & 30.78 & hard tail \\
\hline 274 & 13 & -- & ----- & ----- & 30.15 & no-fit \\
\hline 275 & 63 & 2.14 & $21.22 \pm 0.95$ & $63.93 \pm * * * *$ & 30.76 & hard tail \\
\hline 276 & 46 & 0.78 & $21.06 \pm 0.66$ & $1.55 \pm 0.37$ & 30.49 & fitted \\
\hline 277 & 139 & 1.29 & $22.76 \pm 0.19$ & $47.03 \pm * * * *$ & 31.80 & hard tail \\
\hline 278 & 85 & 1.01 & $21.72 \pm 0.23$ & $2.82 \pm 1.48$ & 30.94 & fitted \\
\hline 279 & 25 & 0.67 & $21.91 \pm 0.91$ & $1.64 \pm 2.26$ & 30.46 & fitted \\
\hline 280 & 3 & -- & ----- & ----- & 29.34 & no-fit \\
\hline 281 & 70 & 1.48 & $21.67 \pm 0.40$ & $0.40 \pm 0.27$ & 31.17 & $\operatorname{Tr} 16-21$ \\
\hline 282 & 46 & 0.65 & $21.52 \pm 0.73$ & $3.32 \pm 3.55$ & 30.70 & fitted \\
\hline 283 & 44 & 0.96 & $21.40 \pm 0.32$ & $2.43 \pm 1.40$ & 30.34 & fitted \\
\hline 284 & 22 & 0.69 & $22.22 \pm 0.21$ & $0.76 \pm 0.46$ & 31.00 & fitted \\
\hline 285 & 31 & 1.12 & $22.24 \pm 0.59$ & $64.00 \pm * * * *$ & 30.64 & hard tail \\
\hline 286 & 6 & -- & ----- & ----- & 29.82 & $\operatorname{Tr} 16-14$ \\
\hline 287 & 31 & 0.95 & $22.47 \pm 0.31$ & $1.23 \pm 0.84$ & 31.08 & fitted \\
\hline 288 & 9 & -- & ----- & ----- & 30.01 & no-fit \\
\hline 289 & 46 & 0.70 & $21.28 \pm 0.29$ & $1.33 \pm 0.18$ & 30.29 & fitted \\
\hline 290 & 37 & 0.49 & $21.99 \pm 0.17$ & $8.15 \pm * * * *$ & 30.42 & hard tail \\
\hline 291 & 16 & -- & ----- & ----- & 30.24 & no-fit \\
\hline 292 & 8 & -- & ----- & ----- & 29.96 & no-fit \\
\hline 293 & 30 & 0.62 & $21.44 \pm 0.71$ & $6.89 \pm * * * *$ & 30.50 & fitted \\
\hline 294 & 27 & 0.46 & $21.82 \pm 0.17$ & $3.39 \pm 2.80$ & 30.26 & fitted \\
\hline 295 & 23 & 0.48 & $22.31 \pm 0.44$ & $64.00 \pm * * * *$ & 30.69 & hard tail \\
\hline 296 & 34 & 0.77 & $21.95 \pm 0.21$ & $7.58 \pm * * * *$ & 30.29 & fitted \\
\hline 297 & 40 & 1.48 & $21.09 \pm 1.43$ & $4.72 \pm 5.84$ & 30.41 & fitted \\
\hline 298 & 14 & -- & ----- & ----- & 32.49 & fitted \\
\hline 299 & 45 & 0.54 & $22.09 \pm 0.17$ & $0.17 \pm 0.44$ & 32.83 & fitted \\
\hline 300 & 9 & -- & ----- & ----- & 30.09 & no-fit \\
\hline 301 & 107 & 0.80 & $21.70 \pm 0.28$ & $1.72 \pm 0.60$ & 31.15 & fitted \\
\hline 302 & 67 & 0.66 & $21.78 \pm 0.18$ & $0.76 \pm 0.18$ & 31.24 & fitted \\
\hline 303 & 11 & -- & ----- & ----- & 30.07 & no-fit \\
\hline 304 & 119 & 0.40 & $22.02 \pm 0.13$ & $1.10 \pm 0.21$ & 31.37 & fitted \\
\hline 305 & 22 & 0.42 & $21.00 \pm 2.33$ & $62.87 \pm * * * *$ & 30.31 & hard tail \\
\hline 306 & 10 & -- & ----- & ----- & 30.05 & no-fit \\
\hline 307 & 27 & 1.16 & $21.96 \pm 0.33$ & $0.98 \pm 0.49$ & 30.95 & fitted \\
\hline 308 & 29 & 0.41 & $21.68 \pm 0.15$ & $1.61 \pm 0.41$ & 30.15 & fitted \\
\hline 309 & 16 & -- & ----- & ----- & 30.24 & no-fit \\
\hline 310 & 3 & -- & ----- & ----- & 29.40 & no-fit \\
\hline 311 & 108 & 2.11 & $21.44 \pm 0.28$ & $4.28 \pm 2.14$ & 30.96 & fitted \\
\hline 312 & 5 & -- & ----- & ----- & 29.75 & no-fit \\
\hline 313 & 14 & -- & ----- & ----- & 30.18 & no-fit \\
\hline 314 & 31 & 0.52 & $21.21 \pm 0.45$ & $2.25 \pm 1.34$ & 30.19 & fitted \\
\hline 315 & 18 & -- & ----- & ----- & 30.30 & no-fit \\
\hline 316 & 24 & 0.68 & $21.60 \pm 0.75$ & $1.04 \pm 0.49$ & 30.29 & fitted \\
\hline 317 & 27 & 0.55 & $21.81 \pm 0.67$ & $1.26 \pm 0.83$ & 30.46 & fitted \\
\hline 318 & 19 & -- & ----- & ----- & 30.31 & no-fit \\
\hline 319 & 9 & -- & ----- & ----- & 30.21 & no-fit \\
\hline 320 & 9 & -- & ----- & ----- & 30.00 & no-fit \\
\hline 321 & 6 & -- & ----- & ----- & 30.00 & no-fit \\
\hline 322 & 128 & 0.90 & $22.16 \pm 0.15$ & $2.50 \pm 0.93$ & 31.44 & fitted \\
\hline 323 & 18 & -- & ----- & ----- & 30.30 & no-fit \\
\hline 324 & 14 & -- & ----- & ----- & 30.18 & no-fit \\
\hline 325 & 70 & 1.35 & $21.93 \pm 0.16$ & $0.60 \pm 0.21$ & 31.20 & fitted \\
\hline
\end{tabular}

Table 3. continued.

\begin{tabular}{|c|c|c|c|c|c|c|}
\hline $\begin{array}{l}N_{x} \\
\#\end{array}$ & $\begin{array}{l}\text { Cnts. } \\
\text { (ph) }\end{array}$ & $\begin{array}{l}\text { Stat. } \\
\left(\chi_{v}^{2}\right)\end{array}$ & $\begin{array}{l}\log \left(N_{\mathrm{H}}\right) \\
\left(\mathrm{cm}^{-2}\right)\end{array}$ & $\begin{array}{l}k T \\
(\mathrm{keV})\end{array}$ & $\begin{array}{l}\log \left(L_{\mathrm{x}}\right) \\
(\mathrm{erg} / \mathrm{s})\end{array}$ & flag \\
\hline 326 & 20 & 0.83 & $21.00 \pm 1.54$ & $36.73 \pm * * * *$ & 30.22 & hard tail \\
\hline 327 & 21 & 1.12 & $21.87 \pm 0.48$ & $0.87 \pm 0.55$ & 30.35 & fitted \\
\hline 328 & 5 & -- & ----- & ----- & 29.77 & no-fit \\
\hline 329 & 6 & -- & ----- & ----- & 29.81 & no-fit \\
\hline 330 & 25 & 0.52 & $21.62 \pm 0.59$ & $0.87 \pm 0.43$ & 30.35 & fitted \\
\hline 331 & 22 & 0.32 & $22.03 \pm 0.36$ & $1.12 \pm 0.63$ & 30.62 & fitted \\
\hline 332 & 26 & -- & ----- & ----- & 30.45 & no-fit \\
\hline 333 & 22 & 0.55 & $21.47 \pm 0.29$ & $1.66 \pm 0.50$ & 30.02 & fitted \\
\hline 334 & 18 & -- & ----- & ----- & 30.28 & no-fit \\
\hline 335 & 7 & -- & ----- & ----- & 29.87 & no-fit \\
\hline 336 & 13 & -- & ----- & ----- & 30.15 & no-fit \\
\hline 337 & 28 & 0.44 & $21.54 \pm 0.58$ & $1.90 \pm 1.09$ & 30.37 & fitted \\
\hline 338 & 8 & -- & ----- & ----- & 29.98 & no-fit \\
\hline 339 & 31 & 0.75 & $21.44 \pm 0.53$ & $1.54 \pm 0.59$ & 30.34 & fitted \\
\hline 340 & 25 & 1.11 & $21.76 \pm 0.33$ & $34.91 \pm * * * *$ & 30.26 & hard tail \\
\hline 341 & 29 & 0.86 & $21.95 \pm 0.52$ & $64.00 \pm * * * *$ & 30.53 & hard tail \\
\hline 342 & 52 & 0.86 & $20.92 \pm 0.86$ & $1.99 \pm 0.63$ & 30.45 & fitted \\
\hline 343 & 4 & -- & ----- & ----- & 29.69 & no-fit \\
\hline 344 & 10 & -- & ----- & ----- & 30.05 & no-fit \\
\hline 345 & 48 & 0.53 & $21.28 \pm 0.44$ & $1.63 \pm 0.38$ & 30.44 & fitted \\
\hline 346 & 8 & -- & ----- & ----- & 29.96 & no-fit \\
\hline 347 & 17 & -- & ----- & ----- & 30.26 & no-fit \\
\hline 348 & 22 & 0.65 & $21.90 \pm 0.15$ & $3.63 \pm 4.58$ & 30.07 & fitted \\
\hline 349 & 16 & -- & ----- & ----- & 30.24 & no-fit \\
\hline 350 & 73 & 0.71 & $21.88 \pm 0.17$ & $1.05 \pm 0.21$ & 31.01 & fitted \\
\hline 351 & 147 & 0.75 & $21.49 \pm 0.19$ & $2.35 \pm 0.63$ & 31.08 & fitted \\
\hline 352 & 1073 & 1.11 & $21.55 \pm 0.57$ & $0.60 \pm 0.30$ & 32.14 & CPD-592600 \\
\hline 353 & 13 & -- & ----- & ----- & 30.16 & no-fit \\
\hline 354 & 46 & 0.71 & $21.67 \pm 0.44$ & $1.37 \pm 0.55$ & 30.60 & fitted \\
\hline 355 & 33 & 0.65 & $21.08 \pm 0.81$ & $64.00 \pm * * * *$ & 30.41 & hard tail \\
\hline 356 & 13 & -- & ----- & ----- & 30.16 & no-fit \\
\hline 357 & 73 & 0.43 & $21.53 \pm 0.28$ & $1.57 \pm 0.33$ & 30.81 & fitted \\
\hline 358 & 9 & -- & ----- & ----- & 30.00 & no-fit \\
\hline 359 & 8 & -- & ----- & ----- & 29.96 & no-fit \\
\hline 360 & 7 & -- & ----- & ----- & 29.87 & no-fit \\
\hline 361 & 9 & -- & ----- & ----- & 30.00 & no-fit \\
\hline 362 & 23 & 0.92 & $22.13 \pm 0.56$ & $2.07 \pm 2.58$ & 30.51 & fitted \\
\hline 363 & 4 & -- & ----- & ----- & 29.65 & no-fit \\
\hline 364 & 18 & -- & ----- & ----- & 30.29 & no-fit \\
\hline 365 & 10 & -- & ----- & ----- & 30.05 & no-fit \\
\hline 366 & 14 & -- & ----- & ----- & 30.18 & no-fit \\
\hline 367 & 12 & -- & ----- & ----- & 30.13 & no-fit \\
\hline 368 & 14 & -- & ----- & ----- & 30.20 & no-fit \\
\hline 369 & 9 & -- & ----- & ----- & 30.01 & no-fit \\
\hline 370 & 57 & 0.45 & $21.82 \pm 0.26$ & $2.43 \pm 1.36$ & 30.82 & fitted \\
\hline 371 & 7 & -- & ----- & ----- & 29.90 & no-fit \\
\hline 372 & 22 & 0.41 & $22.46 \pm 0.28$ & $1.03 \pm 0.67$ & 31.44 & fitted \\
\hline 373 & 6 & -- & ----- & ----- & 29.87 & no-fit \\
\hline 374 & 7 & -- & ----- & ----- & 29.87 & no-fit \\
\hline 375 & 26 & 0.24 & $21.64 \pm 0.54$ & $2.51 \pm 2.44$ & 30.42 & fitted \\
\hline 376 & 32 & 0.42 & $21.07 \pm 0.86$ & $1.71 \pm 0.49$ & 30.27 & fitted \\
\hline 377 & 10 & -- & ----- & ----- & 30.06 & no-fit \\
\hline 378 & 36 & 0.32 & $21.83 \pm 0.39$ & $1.72 \pm 0.75$ & 30.71 & fitted \\
\hline 379 & 66 & 0.58 & $21.59 \pm 0.30$ & $2.56 \pm 1.17$ & 31.08 & fitted \\
\hline 380 & 7 & -- & ----- & ----- & 29.91 & no-fit \\
\hline 381 & 21 & 0.66 & $21.64 \pm 0.66$ & $6.62 \pm * * * *$ & 30.68 & fitted \\
\hline 382 & 32 & 0.67 & $22.09 \pm 0.39$ & $2.36 \pm 1.81$ & 30.92 & fitted \\
\hline 383 & 49 & 0.76 & $21.57 \pm 0.44$ & $1.19 \pm 0.36$ & 30.81 & fitted \\
\hline 384 & 17 & -- & ----- & ----- & 30.26 & no-fit \\
\hline 385 & 32 & 0.85 & $22.29 \pm 0.56$ & $63.36 \pm * * * *$ & 30.82 & hard tail \\
\hline 386 & 21 & 0.81 & $22.18 \pm 0.48$ & $64.00 \pm * * * *$ & 30.61 & hard tail \\
\hline 387 & 16 & -- & ----- & ----- & 30.24 & no-fit \\
\hline 388 & 53 & 1.07 & $20.84 \pm 1.08$ & $3.83 \pm 3.51$ & 30.49 & fitted \\
\hline 389 & 8 & -- & ----- & ----- & 29.94 & no-fit \\
\hline 390 & 9 & -- & ----- & ----- & 30.00 & no-fit \\
\hline
\end{tabular}


Table 3. continued.

\begin{tabular}{|c|c|c|c|c|c|c|}
\hline $\begin{array}{l}N_{x} \\
\#\end{array}$ & $\begin{array}{l}\text { Cnts. } \\
\text { (ph) }\end{array}$ & $\begin{array}{l}\text { Stat. } \\
\left(\chi_{v}^{2}\right)\end{array}$ & $\begin{array}{l}\log \left(N_{\mathrm{H}}\right) \\
\left(\mathrm{cm}^{-2}\right)\end{array}$ & $\begin{array}{l}k T \\
(\mathrm{keV})\end{array}$ & $\begin{array}{l}\log \left(L_{\mathrm{x}}\right) \\
(\mathrm{erg} / \mathrm{s})\end{array}$ & flag \\
\hline 391 & 18 & -- & ----- & ----- & 30.30 & no-fit \\
\hline 392 & 46 & 0.83 & $21.06 \pm 0.67$ & $1.70 \pm 0.36$ & 30.71 & fitted \\
\hline 393 & 31 & 0.94 & $21.94 \pm 0.71$ & $1.62 \pm 1.61$ & 30.68 & fitted \\
\hline 394 & 7 & -- & ----- & ----- & 29.92 & no-fit \\
\hline 395 & 36 & 0.46 & $22.39 \pm 0.29$ & $6.18 \pm 9.66$ & 31.06 & fitted \\
\hline 396 & 19 & -- & ----- & ----- & 30.32 & no-fit \\
\hline 397 & 19 & -- & ----- & ----- & 30.31 & no-fit \\
\hline 398 & 18 & -- & ----- & ----- & 30.29 & no-fit \\
\hline 399 & 5 & -- & ----- & ----- & 29.77 & no-fit \\
\hline 400 & 44 & 0.80 & $21.16 \pm 0.58$ & $1.39 \pm 0.39$ & 30.77 & fitted \\
\hline 401 & 81 & 0.76 & $21.17 \pm 0.57$ & $2.65 \pm 1.10$ & 30.79 & fitted \\
\hline 402 & 11 & -- & ----- & ----- & 30.10 & no-fit \\
\hline 403 & 42 & 1.12 & $21.17 \pm 0.79$ & $47.19 \pm * * * *$ & 30.58 & hard tail \\
\hline 404 & 16 & -- & ----- & ----- & 30.24 & no-fit \\
\hline 405 & 27 & 0.61 & $22.26 \pm 0.52$ & $2.06 \pm 2.48$ & 30.62 & fitted \\
\hline 406 & 66 & 0.89 & $21.84 \pm 0.23$ & $2.91 \pm 1.68$ & 30.91 & fitted \\
\hline 407 & 204 & 0.54 & $21.33 \pm 2.33$ & $0.64 \pm 0.82$ & 31.49 & CPD-592603C \\
\hline 408 & 51 & 1.05 & $21.47 \pm 0.52$ & $0.81 \pm 0.21$ & 30.69 & fitted \\
\hline 409 & 33 & 0.76 & $23.01 \pm 0.40$ & $2.41 \pm 3.02$ & 31.38 & fitted \\
\hline 410 & 12 & -- & ----- & ----- & 30.11 & no-fit \\
\hline 411 & 19 & -- & ----- & ----- & 30.32 & no-fit \\
\hline 412 & 26 & 0.85 & $21.94 \pm 0.56$ & $2.00 \pm 2.02$ & 30.77 & fitted \\
\hline 413 & 23 & 0.49 & $21.72 \pm 0.77$ & $1.72 \pm 1.58$ & 30.70 & fitted \\
\hline 414 & 24 & 0.79 & $21.27 \pm 0.72$ & $1.84 \pm 1.20$ & 30.60 & fitted \\
\hline 415 & 14 & -- & ----- & ----- & 30.18 & no-fit \\
\hline 416 & 19 & -- & ----- & ----- & 31.10 & fitted \\
\hline 417 & 106 & 0.88 & $21.58 \pm 0.22$ & $1.86 \pm 0.46$ & 30.95 & fitted \\
\hline 418 & 11 & -- & ----- & ----- & 30.09 & no-fit \\
\hline 419 & 123 & 0.80 & $21.46 \pm 0.22$ & $2.04 \pm 0.54$ & 30.99 & fitted \\
\hline 420 & 50 & 0.35 & $21.81 \pm 0.30$ & $2.96 \pm 2.02$ & 30.79 & fitted \\
\hline 421 & 5 & -- & ----- & ----- & 29.78 & no-fit \\
\hline 422 & 5 & -- & ----- & ----- & 29.76 & no-fit \\
\hline 423 & 5 & -- & ----- & ----- & 29.75 & no-fit \\
\hline 424 & 28 & 0.79 & $21.42 \pm 0.88$ & $44.83 \pm * * * *$ & 30.63 & hard tail \\
\hline 425 & 30 & 0.79 & $22.35 \pm 0.21$ & $0.67 \pm 0.47$ & 31.41 & fitted \\
\hline 426 & 16 & -- & ----- & ----- & 30.23 & no-fit \\
\hline 427 & 25 & 0.52 & $21.72 \pm 0.53$ & $2.56 \pm 2.81$ & 30.75 & fitted \\
\hline 428 & 10 & -- & ----- & ----- & 30.23 & no-fit \\
\hline 429 & 27 & 0.65 & $21.89 \pm 0.17$ & $5.06 \pm * * * *$ & 30.26 & fitted \\
\hline 430 & 27 & 0.67 & $21.83 \pm 0.75$ & $1.76 \pm 1.29$ & 30.37 & fitted \\
\hline 431 & 67 & 1.36 & $21.27 \pm 0.55$ & $42.71 \pm * * * *$ & 30.81 & hard tail \\
\hline 432 & 73 & 0.55 & $21.07 \pm 0.67$ & $13.03 \pm * * * *$ & 30.92 & hard tail \\
\hline 433 & 9 & -- & ----- & ----- & 30.01 & no-fit \\
\hline 434 & 32 & 0.84 & $21.53 \pm 0.70$ & $3.49 \pm 4.02$ & 30.31 & fitted \\
\hline 435 & 12 & -- & ----- & ----- & 30.12 & no-fit \\
\hline 436 & 10 & -- & ----- & ----- & 30.06 & no-fit \\
\hline 437 & 63 & 1.30 & $22.00 \pm 0.29$ & $3.07 \pm 3.00$ & 30.97 & fitted \\
\hline 438 & 208 & 1.11 & $21.47 \pm 0.19$ & $10.51 \pm 7.20$ & 31.33 & hard tail \\
\hline 439 & 66 & 1.44 & $21.43 \pm 0.35$ & $3.65 \pm 2.19$ & 30.69 & fitted \\
\hline 440 & 51 & 1.08 & $21.33 \pm 0.53$ & $3.40 \pm 2.71$ & 30.66 & fitted \\
\hline 441 & 76 & 0.55 & $21.16 \pm 0.40$ & $2.26 \pm 1.10$ & 30.73 & fitted \\
\hline 442 & 13 & -- & ----- & ----- & 30.17 & no-fit \\
\hline 443 & 9 & -- & ----- & ----- & 29.99 & no-fit \\
\hline 444 & 15 & -- & ----- & ----- & 30.21 & no-fit \\
\hline 445 & 5 & -- & ----- & ----- & 30.13 & no-fit \\
\hline 446 & 104 & 1.14 & $21.95 \pm 0.18$ & $2.40 \pm 0.99$ & 31.14 & fitted \\
\hline 447 & 167 & 1.41 & $21.26 \pm 2.73$ & $4.87 \pm 2.30$ & 31.10 & fitted \\
\hline 448 & 44 & 1.25 & $21.58 \pm 0.46$ & $2.17 \pm 1.64$ & 30.49 & fitted \\
\hline 449 & 63 & 0.69 & $21.17 \pm 0.52$ & $4.50 \pm 4.60$ & 30.70 & fitted \\
\hline 450 & 136 & 0.70 & $21.85 \pm 0.23$ & $2.09 \pm 0.69$ & 31.23 & fitted \\
\hline 451 & 16 & -- & ----- & ----- & 30.25 & no-fit \\
\hline 452 & 8 & -- & ----- & ----- & 29.94 & no-fit \\
\hline 453 & 51 & 1.12 & $22.13 \pm 0.37$ & $1.93 \pm 1.69$ & 30.95 & fitted \\
\hline 454 & 12 & -- & ----- & ----- & 30.12 & no-fit \\
\hline 455 & 5 & -- & ----- & ----- & 29.77 & no-fit \\
\hline
\end{tabular}

Table 3. continued.

\begin{tabular}{|c|c|c|c|c|c|c|}
\hline $\begin{array}{l}N_{x} \\
\#\end{array}$ & $\begin{array}{l}\text { Cnts. } \\
\text { (ph) }\end{array}$ & $\begin{array}{l}\text { Stat. } \\
\left(\chi_{v}^{2}\right)\end{array}$ & $\begin{array}{l}\log \left(N_{\mathrm{H}}\right) \\
\left(\mathrm{cm}^{-2}\right)\end{array}$ & $\begin{array}{l}k T \\
(\mathrm{keV})\end{array}$ & $\begin{array}{l}\log \left(L_{\mathrm{x}}\right) \\
(\mathrm{erg} / \mathrm{s})\end{array}$ & flag \\
\hline 456 & 7 & -- & ----- & ----- & 29.90 & no-fit \\
\hline 457 & 19 & -- & ----- & ----- & 30.32 & no-fit \\
\hline 458 & 53 & 0.77 & $21.63 \pm 0.32$ & $1.72 \pm 0.58$ & 30.61 & fitted \\
\hline 459 & 61 & 0.91 & $22.63 \pm 0.22$ & $64.00 \pm * * * *$ & 31.28 & hard tail \\
\hline 460 & 10 & -- & ----- & ----- & 30.06 & no-fit \\
\hline 461 & 18 & -- & ----- & ----- & 30.29 & no-fit \\
\hline 462 & 15 & -- & ----- & ----- & 30.21 & no-fit \\
\hline 463 & 7 & -- & ----- & ----- & 29.92 & no-fit \\
\hline 464 & 9 & -- & ----- & ----- & 30.02 & no-fit \\
\hline 465 & 9 & -- & ----- & ----- & 29.99 & no-fit \\
\hline 466 & 6 & -- & ----- & ----- & 29.84 & no-fit \\
\hline 467 & 33 & 0.91 & $21.74 \pm 0.53$ & $1.72 \pm 1.07$ & 30.39 & fitted \\
\hline 468 & 435 & 0.47 & $21.63 \pm 1.08$ & $20.08 \pm * * * *$ & 31.73 & hard tail \\
\hline 469 & 25 & 0.85 & $21.86 \pm 0.19$ & $0.89 \pm 0.30$ & 29.92 & fitted \\
\hline 470 & 49 & 0.61 & $21.63 \pm 0.43$ & $1.12 \pm 0.42$ & 30.58 & fitted \\
\hline 471 & 7 & -- & ----- & ----- & 29.89 & no-fit \\
\hline 472 & 52 & 1.16 & $22.48 \pm 0.41$ & $13.66 \pm * * * *$ & 31.20 & hard tail \\
\hline 473 & 53 & 0.34 & $21.16 \pm 0.63$ & $7.32 \pm 9.24$ & 30.97 & fitted \\
\hline 474 & 12 & -- & ----- & ----- & 30.12 & no-fit \\
\hline 475 & 5 & -- & ----- & ----- & 29.77 & no-fit \\
\hline 476 & 26 & 0.42 & $22.10 \pm 0.24$ & $1.04 \pm 0.50$ & 30.79 & fitted \\
\hline 477 & 24 & 0.79 & $21.47 \pm 1.04$ & $0.96 \pm 0.40$ & 29.92 & fitted \\
\hline 478 & 17 & -- & ----- & ----- & 30.28 & no-fit \\
\hline 479 & 16 & -- & ----- & ----- & 30.24 & no-fit \\
\hline 480 & 27 & -- & ----- & ----- & 30.47 & no-fit \\
\hline 481 & 25 & 0.56 & $22.15 \pm 0.48$ & $1.67 \pm 1.44$ & 30.63 & fitted \\
\hline 482 & 64 & 0.69 & $21.52 \pm 0.29$ & $2.32 \pm 0.84$ & 30.86 & fitted \\
\hline 483 & 6 & -- & ----- & ----- & 29.83 & no-fit \\
\hline 484 & 12 & -- & ----- & ----- & 30.13 & no-fit \\
\hline 485 & 15 & -- & ----- & ----- & 30.21 & no-fit \\
\hline 486 & 32 & 0.56 & $22.01 \pm 0.23$ & $0.86 \pm 0.40$ & 30.82 & fitted \\
\hline 487 & 12 & -- & ----- & ----- & 30.13 & no-fit \\
\hline 488 & 6 & -- & ----- & ----- & 29.81 & no-fit \\
\hline 489 & 159 & 1.14 & $21.36 \pm 2.08$ & $2.87 \pm 0.88$ & 31.10 & $\operatorname{Tr} 16-5$ \\
\hline 490 & 22 & 1.07 & $21.57 \pm 1.44$ & $1.36 \pm 1.33$ & 30.06 & fitted \\
\hline 491 & 52 & 0.57 & $21.37 \pm 0.52$ & $9.89 \pm * * * *$ & 30.72 & hard tail \\
\hline 492 & 7 & -- & ----- & ----- & 29.89 & no-fit \\
\hline 493 & 3 & -- & ----- & ----- & 29.18 & no-fit \\
\hline 494 & 9 & -- & ----- & ----- & 30.00 & no-fit \\
\hline 495 & 54 & 0.79 & $22.34 \pm 0.17$ & $1.15 \pm 0.42$ & 31.37 & fitted \\
\hline 496 & 75 & 0.97 & $21.34 \pm 0.34$ & $2.10 \pm 0.61$ & 30.69 & fitted \\
\hline 497 & 63 & 1.17 & $21.95 \pm 0.13$ & $0.75 \pm 0.21$ & 31.15 & fitted \\
\hline 498 & 10 & -- & ----- & ----- & 30.03 & no-fit \\
\hline 499 & 52 & 0.38 & $22.57 \pm 0.16$ & $0.85 \pm 0.42$ & 31.95 & fitted \\
\hline 500 & 20 & 0.25 & $21.77 \pm 0.19$ & $2.33 \pm 1.52$ & 30.09 & fitted \\
\hline 501 & 29 & 1.34 & $21.85 \pm 0.51$ & $1.37 \pm 0.53$ & 29.99 & fitted \\
\hline 502 & 16 & -- & ----- & ----- & 30.24 & no-fit \\
\hline 503 & 474 & 1.55 & $21.57 \pm 0.95$ & $3.08 \pm 0.56$ & 31.63 & fitted \\
\hline 504 & 19 & -- & ----- & ----- & 30.31 & no-fit \\
\hline 505 & 5 & -- & ----- & ----- & 29.77 & no-fit \\
\hline 506 & 8 & -- & ----- & ----- & 29.94 & no-fit \\
\hline 507 & 122 & 0.76 & $22.08 \pm 0.18$ & $1.37 \pm 0.43$ & 31.40 & fitted \\
\hline 508 & 15 & -- & ----- & ----- & 30.22 & no-fit \\
\hline 509 & 28 & 0.92 & $21.79 \pm 0.69$ & $0.34 \pm 0.58$ & 30.85 & fitted \\
\hline 510 & 14 & -- & ----- & ----- & 30.20 & no-fit \\
\hline 511 & 9 & -- & ----- & ----- & 30.01 & no-fit \\
\hline 512 & 15 & -- & ----- & ----- & 30.20 & no-fit \\
\hline 513 & 107 & 0.49 & $21.64 \pm 0.24$ & $1.72 \pm 0.43$ & 30.99 & fitted \\
\hline 514 & 13 & -- & ----- & ----- & 30.15 & no-fit \\
\hline 515 & 15 & -- & ----- & ----- & 30.21 & no-fit \\
\hline 516 & 16 & -- & ----- & ----- & 30.25 & no-fit \\
\hline 517 & 13 & -- & ----- & ----- & 30.16 & no-fit \\
\hline 518 & 43 & 0.50 & $22.02 \pm 0.40$ & $1.37 \pm 0.86$ & 30.95 & fitted \\
\hline 519 & 42 & 0.87 & $22.33 \pm 0.29$ & $1.95 \pm 1.18$ & 31.03 & fitted \\
\hline 520 & 16 & -- & ----- & ----- & 30.25 & no-fit \\
\hline
\end{tabular}


Table 3. continued.

\begin{tabular}{|c|c|c|c|c|c|c|}
\hline $\begin{array}{l}N_{x} \\
\#\end{array}$ & $\begin{array}{l}\text { Cnts. } \\
(\mathrm{ph})\end{array}$ & $\begin{array}{l}\text { Stat. } \\
\left(\chi_{v}^{2}\right)\end{array}$ & $\begin{array}{l}\log \left(N_{\mathrm{H}}\right) \\
\left(\mathrm{cm}^{-2}\right)\end{array}$ & $\begin{array}{l}k T \\
(\mathrm{keV})\end{array}$ & $\begin{array}{l}\log \left(L_{\mathrm{x}}\right) \\
(\mathrm{erg} / \mathrm{s})\end{array}$ & flag \\
\hline 521 & 50 & 0.30 & $21.78 \pm 0.48$ & $1.37 \pm 0.71$ & 30.78 & fitted \\
\hline 522 & 21 & 0.94 & $22.20 \pm 0.55$ & $2.07 \pm 2.51$ & 30.44 & fitted \\
\hline 523 & 91 & 0.80 & $22.05 \pm 0.18$ & $3.45 \pm 1.72$ & 31.15 & fitted \\
\hline 524 & 1367 & 1.01 & $21.85 \pm 0.50$ & $7.20 \pm 1.70$ & 32.32 & fitted \\
\hline 525 & 19 & -- & ----- & ----- & 30.33 & no-fit \\
\hline 526 & 103 & 0.94 & $21.73 \pm 0.23$ & $2.17 \pm 0.80$ & 31.02 & fitted \\
\hline 527 & 29 & 0.73 & $21.69 \pm 0.55$ & $1.72 \pm 0.86$ & 30.37 & fitted \\
\hline 528 & 7 & -- & ----- & ----- & 29.88 & no-fit \\
\hline 529 & 17 & -- & ----- & ----- & 30.26 & no-fit \\
\hline 530 & 9 & -- & ----- & ----- & 30.00 & no-fit \\
\hline 531 & 148 & 0.45 & $21.68 \pm 0.20$ & $1.44 \pm 0.30$ & 31.15 & fitted \\
\hline 532 & 12 & -- & ----- & ----- & 30.12 & no-fit \\
\hline 533 & 7 & -- & ----- & ----- & 29.88 & no-fit \\
\hline 534 & 121 & 0.88 & $21.54 \pm 0.17$ & $0.60 \pm 0.90$ & 31.33 & fitted \\
\hline 535 & 38 & 0.23 & $21.66 \pm 0.40$ & $1.60 \pm 0.54$ & 30.56 & fitted \\
\hline 536 & 10 & -- & ----- & ----- & 30.06 & no-fit \\
\hline 537 & 104 & 0.74 & $21.12 \pm 0.39$ & $2.37 \pm 0.84$ & 30.85 & fitted \\
\hline 538 & 18 & -- & ----- & ----- & 30.28 & no-fit \\
\hline 539 & 9 & -- & ----- & ----- & 30.02 & no-fit \\
\hline 540 & 41 & 0.69 & $22.23 \pm 0.35$ & $2.45 \pm 1.90$ & 30.91 & fitted \\
\hline 541 & 10 & -- & ----- & ----- & 30.06 & no-fit \\
\hline 542 & 51 & 0.75 & $21.33 \pm 0.51$ & $10.02 \pm * * * *$ & 30.64 & hard tail \\
\hline 543 & 28 & 0.67 & $22.17 \pm 0.45$ & $1.52 \pm 1.27$ & 30.71 & fitted \\
\hline 544 & 8 & -- & ----- & ----- & 29.94 & no-fit \\
\hline 545 & 10 & -- & ----- & ----- & 30.05 & no-fit \\
\hline 546 & 29 & 0.51 & $21.94 \pm 0.47$ & $4.26 \pm 5.08$ & 30.59 & fitted \\
\hline 547 & 66 & 0.36 & $21.64 \pm 0.28$ & $4.79 \pm 3.65$ & 30.89 & fitted \\
\hline 548 & 19 & -- & ----- & ----- & 35.60 & fitted \\
\hline 549 & 30 & 0.45 & $22.11 \pm 0.41$ & $1.59 \pm 1.13$ & 30.76 & fitted \\
\hline 550 & 28 & 0.70 & $22.26 \pm 0.46$ & $1.10 \pm 1.61$ & 31.04 & fitted \\
\hline 551 & 39 & 0.87 & $21.88 \pm 0.12$ & $1.37 \pm 0.36$ & 30.24 & fitted \\
\hline 552 & 155 & 0.77 & $21.64 \pm 0.18$ & $2.40 \pm 0.64$ & 31.19 & fitted \\
\hline 553 & 66 & 0.87 & $21.38 \pm 0.35$ & $2.72 \pm 1.14$ & 30.76 & fitted \\
\hline 554 & 30 & 0.65 & $21.80 \pm 0.32$ & $0.86 \pm 0.39$ & 30.62 & fitted \\
\hline 555 & 35 & 0.69 & $22.02 \pm 0.21$ & $0.86 \pm 0.43$ & 30.82 & fitted \\
\hline 556 & 5 & -- & ----- & ----- & 29.80 & no-fit \\
\hline 557 & 7 & -- & ----- & ----- & 29.93 & no-fit \\
\hline 558 & 32 & 0.72 & $21.97 \pm 0.18$ & $0.67 \pm 0.35$ & 30.99 & fitted \\
\hline 559 & 33 & 0.61 & $21.74 \pm 0.21$ & $5.89 \pm * * * *$ & 30.47 & fitted \\
\hline 560 & 9 & -- & ----- & ----- & 29.98 & no-fit \\
\hline 561 & 10 & -- & ----- & ----- & 30.04 & no-fit \\
\hline 562 & 28 & 0.39 & $21.50 \pm 0.26$ & $3.61 \pm 5.90$ & 30.34 & fitted \\
\hline 563 & 27 & 0.55 & $21.65 \pm 0.61$ & $1.37 \pm 0.64$ & 30.57 & fitted \\
\hline 564 & 251 & 1.17 & $21.95 \pm 0.15$ & $17.19 \pm * * * *$ & 31.64 & hard tail \\
\hline 565 & 24 & 0.57 & $21.60 \pm 0.72$ & $1.23 \pm 0.64$ & 30.28 & fitted \\
\hline 566 & 10 & -- & ----- & ----- & 30.04 & no-fit \\
\hline 567 & 41 & 0.82 & $21.15 \pm 2.37$ & $63.13 \pm * * * *$ & 30.50 & hard tail \\
\hline 568 & 19 & -- & ----- & ----- & 30.30 & no-fit \\
\hline 569 & 79 & 0.64 & $21.78 \pm 0.25$ & $1.39 \pm 0.42$ & 30.91 & fitted \\
\hline 570 & 12 & -- & ----- & ----- & 30.13 & no-fit \\
\hline 571 & 6 & -- & ----- & ----- & 29.84 & no-fit \\
\hline 572 & 53 & 1.15 & $21.46 \pm 0.44$ & $3.27 \pm 2.05$ & 30.61 & fitted \\
\hline 573 & 31 & 0.91 & $21.27 \pm 0.84$ & $2.16 \pm 1.68$ & 30.21 & fitted \\
\hline 574 & 5 & -- & ----- & ----- & 29.82 & no-fit \\
\hline 575 & 7 & -- & ----- & ----- & 29.93 & no-fit \\
\hline 576 & 9 & -- & ----- & ----- & 30.01 & no-fit \\
\hline 577 & 21 & 0.46 & $21.19 \pm 1.27$ & $0.91 \pm 0.38$ & 30.10 & fitted \\
\hline 578 & 18 & -- & ----- & ----- & 30.30 & no-fit \\
\hline 579 & 6 & -- & ----- & ----- & 29.85 & no-fit \\
\hline 580 & 9 & -- & ----- & ----- & 29.99 & no-fit \\
\hline 581 & 83 & 1.18 & $21.22 \pm 0.41$ & $3.92 \pm 1.93$ & 31.74 & fitted \\
\hline 582 & 16 & -- & ----- & ----- & 31.58 & fitted \\
\hline 583 & 25 & 0.45 & $22.56 \pm 0.40$ & $64.00 \pm * * * *$ & 32.79 & hard tail \\
\hline 584 & 17 & -- & ----- & ----- & 30.28 & no-fit \\
\hline 585 & 52 & 1.11 & $21.07 \pm 0.81$ & $2.73 \pm 1.90$ & 30.42 & fitted \\
\hline
\end{tabular}

Table 3. continued.

\begin{tabular}{|c|c|c|c|c|c|c|}
\hline $\begin{array}{l}N_{x} \\
\#\end{array}$ & $\begin{array}{l}\text { Cnts. } \\
(\mathrm{ph})\end{array}$ & $\begin{array}{l}\text { Stat. } \\
\left(\chi_{v}^{2}\right)\end{array}$ & $\begin{array}{l}\log \left(N_{\mathrm{H}}\right) \\
\left(\mathrm{cm}^{-2}\right)\end{array}$ & $\begin{array}{l}k T \\
(\mathrm{keV})\end{array}$ & $\begin{array}{l}\log \left(L_{\mathrm{x}}\right) \\
(\mathrm{erg} / \mathrm{s})\end{array}$ & flag \\
\hline 586 & 6 & -- & ----- & ----- & 29.84 & no-fit \\
\hline 587 & 66 & 0.90 & $21.99 \pm 0.23$ & $1.09 \pm 0.38$ & 31.04 & fitted \\
\hline 588 & 32 & 0.85 & $21.15 \pm 0.93$ & $6.50 \pm 9.21$ & 30.27 & fitted \\
\hline 589 & 38 & 0.72 & $22.04 \pm 0.15$ & $0.58 \pm 0.22$ & 31.31 & fitted \\
\hline 590 & 32 & 0.42 & $21.49 \pm 0.62$ & $1.15 \pm 0.42$ & 30.33 & fitted \\
\hline 591 & 34 & 0.82 & $21.52 \pm 0.53$ & $1.38 \pm 0.75$ & 30.29 & fitted \\
\hline 592 & 3 & -- & ----- & ----- & 28.92 & no-fit \\
\hline 593 & 33 & 0.65 & $21.09 \pm 0.97$ & $3.43 \pm 5.50$ & 30.36 & fitted \\
\hline 594 & 52 & 0.80 & $22.56 \pm 0.21$ & $2.25 \pm 1.55$ & 31.34 & fitted \\
\hline 595 & 12 & -- & ----- & ----- & 30.11 & no-fit \\
\hline 596 & 27 & 0.78 & $21.67 \pm 0.63$ & $3.26 \pm 3.84$ & 30.34 & fitted \\
\hline 597 & 21 & 0.48 & $21.95 \pm 0.13$ & $2.26 \pm 1.68$ & 30.13 & fitted \\
\hline 598 & 5 & -- & ----- & ----- & 29.79 & no-fit \\
\hline 599 & 26 & 0.80 & $21.78 \pm 0.42$ & $9.36 \pm * * * *$ & 30.13 & hard tail \\
\hline 600 & 525 & 1.55 & $21.65 \pm 0.64$ & $0.62 \pm 0.43$ & 31.87 & fitted \\
\hline 601 & 7 & -- & ----- & ----- & 29.90 & no-fit \\
\hline 602 & 39 & 0.56 & $21.29 \pm 0.67$ & $1.16 \pm 0.31$ & 30.37 & fitted \\
\hline 603 & 82 & 0.40 & $21.82 \pm 0.29$ & $1.61 \pm 0.49$ & 30.99 & fitted \\
\hline 604 & 12 & -- & ----- & ----- & 30.14 & no-fit \\
\hline 605 & 99 & 0.72 & $21.63 \pm 0.25$ & $3.84 \pm 2.13$ & 31.01 & fitted \\
\hline 606 & 47 & 0.93 & $21.40 \pm 0.44$ & $9.81 \pm * * * *$ & 30.57 & hard tail \\
\hline 607 & 3 & -- & ----- & ----- & 28.92 & no-fit \\
\hline 608 & 9 & -- & ----- & ----- & 30.18 & no-fit \\
\hline 609 & 13 & -- & ----- & ----- & 30.16 & no-fit \\
\hline 610 & 17 & -- & ----- & ----- & 30.26 & no-fit \\
\hline 611 & 11 & -- & ----- & ----- & 30.09 & no-fit \\
\hline 612 & 18 & -- & ----- & ----- & 30.30 & no-fit \\
\hline 613 & 9 & -- & ----- & ----- & 30.22 & no-fit \\
\hline 614 & 29 & 1.55 & $22.78 \pm 0.23$ & $2.21 \pm 1.71$ & 31.45 & fitted \\
\hline 615 & 8 & -- & ----- & ----- & 29.95 & no-fit \\
\hline 616 & 44 & 0.56 & $22.42 \pm 0.24$ & $34.30 \pm * * * *$ & 31.11 & hard tail \\
\hline 617 & 23 & 0.36 & $21.03 \pm 0.90$ & $1.70 \pm 0.46$ & 32.49 & fitted \\
\hline 618 & 19 & -- & ----- & ----- & 30.32 & no-fit \\
\hline 619 & 422 & 0.99 & $21.23 \pm 2.27$ & $0.51 \pm 0.80$ & 31.52 & fitted \\
\hline 620 & 224 & 1.31 & $21.96 \pm 0.17$ & $2.03 \pm 0.55$ & 31.56 & fitted \\
\hline 621 & 27 & 0.50 & $21.73 \pm 0.47$ & $3.02 \pm 3.67$ & 30.44 & fitted \\
\hline 622 & 17 & -- & ----- & ----- & 30.64 & no-fit \\
\hline 623 & 77 & 0.59 & $21.72 \pm 0.32$ & $3.13 \pm 1.73$ & 30.93 & fitted \\
\hline 624 & 425 & 1.93 & $21.70 \pm 0.14$ & $0.47 \pm 0.65$ & 31.30 & fitted \\
\hline 625 & 5 & -- & ----- & ----- & 29.76 & no-fit \\
\hline 626 & 10 & -- & ----- & ----- & 30.05 & no-fit \\
\hline 627 & 69 & 0.59 & $21.36 \pm 0.33$ & $2.15 \pm 0.57$ & 30.75 & fitted \\
\hline 628 & 34 & 0.64 & $21.25 \pm 0.70$ & $2.56 \pm 1.47$ & 30.39 & fitted \\
\hline 629 & 33 & 0.55 & $21.20 \pm 0.66$ & $1.88 \pm 0.85$ & 30.26 & fitted \\
\hline 630 & 11 & -- & ----- & ----- & 30.07 & no-fit \\
\hline 631 & 34 & 0.45 & $21.24 \pm 0.58$ & $1.83 \pm 0.80$ & 30.30 & fitted \\
\hline 632 & 58 & 0.36 & $21.74 \pm 0.27$ & $1.75 \pm 0.63$ & 30.79 & fitted \\
\hline 633 & 23 & 0.44 & $22.06 \pm 0.43$ & $1.45 \pm 0.99$ & 30.57 & fitted \\
\hline 634 & 11 & -- & ----- & ----- & 30.10 & no-fit \\
\hline 635 & 7 & -- & ----- & ----- & 29.88 & no-fit \\
\hline 636 & 16 & -- & ----- & ----- & 30.24 & no-fit \\
\hline 637 & 18 & -- & ----- & ----- & 30.28 & no-fit \\
\hline 638 & 114 & 0.96 & $21.39 \pm 0.24$ & $2.35 \pm 0.91$ & 30.96 & fitted \\
\hline 639 & 10 & -- & ----- & ----- & 30.06 & no-fit \\
\hline 640 & 12 & -- & ----- & ----- & 30.10 & no-fit \\
\hline 641 & 9 & -- & ----- & ----- & 29.99 & no-fit \\
\hline 642 & 24 & 0.62 & $21.78 \pm 0.69$ & $1.09 \pm 0.76$ & 30.42 & fitted \\
\hline 643 & 16 & -- & ----- & ----- & 30.23 & no-fit \\
\hline 644 & 9 & -- & ----- & ----- & 30.02 & no-fit \\
\hline 645 & 70 & 1.12 & $22.02 \pm 0.23$ & $1.12 \pm 0.37$ & 31.07 & fitted \\
\hline 646 & 6 & -- & ----- & ----- & 29.84 & no-fit \\
\hline 647 & 3 & -- & ----- & ----- & 28.92 & no-fit \\
\hline 648 & 3 & -- & ----- & ----- & 28.92 & no-fit \\
\hline 649 & 17563 & 6.03 & $22.65 \pm 0.01$ & $64.00 \pm * * * *$ & 33.77 & Eta Carina \\
\hline 650 & 92 & 0.92 & $21.93 \pm 0.54$ & $4.96 \pm 2.97$ & 30.90 & fitted \\
\hline
\end{tabular}


Table 3. continued.

\begin{tabular}{|c|c|c|c|c|c|c|}
\hline $\begin{array}{l}N_{x} \\
\#\end{array}$ & $\begin{array}{l}\text { Cnts. } \\
(\mathrm{ph})\end{array}$ & $\begin{array}{l}\text { Stat. } \\
\left(\chi_{v}^{2}\right)\end{array}$ & $\begin{array}{l}\log \left(N_{\mathrm{H}}\right) \\
\left(\mathrm{cm}^{-2}\right)\end{array}$ & $\begin{array}{l}k T \\
(\mathrm{keV})\end{array}$ & $\begin{array}{l}\log \left(L_{\mathrm{x}}\right) \\
(\mathrm{erg} / \mathrm{s})\end{array}$ & flag \\
\hline 651 & 435 & 0.82 & $21.69 \pm 0.96$ & $5.24 \pm 1.28$ & 31.70 & fitted \\
\hline 652 & 14 & -- & ----- & ----- & 30.18 & no-fit \\
\hline 653 & 11 & -- & ----- & ----- & 30.10 & no-fit \\
\hline 654 & 13 & -- & ----- & ----- & 30.16 & no-fit \\
\hline 655 & 18 & -- & ----- & ----- & 30.30 & no-fit \\
\hline 656 & 119 & 0.86 & $21.39 \pm 0.28$ & $2.10 \pm 0.45$ & 30.98 & fitted \\
\hline 657 & 20 & -- & ----- & ----- & 30.33 & no-fit \\
\hline 658 & 50 & 0.67 & $21.91 \pm 0.09$ & $3.01 \pm 2.07$ & 30.47 & fitted \\
\hline 659 & 29 & 0.36 & $21.08 \pm 1.13$ & $21.66 \pm * * * *$ & 30.43 & hard tail \\
\hline 660 & 10 & -- & ----- & ----- & 30.03 & no-fit \\
\hline 661 & 26 & 0.68 & $21.46 \pm 0.72$ & $3.46 \pm 3.80$ & 30.20 & fitted \\
\hline 662 & 29 & 1.19 & $21.23 \pm 0.94$ & $1.81 \pm 1.55$ & 30.19 & fitted \\
\hline 663 & 369 & 1.19 & $21.25 \pm 1.87$ & $3.03 \pm 0.63$ & 31.46 & fitted \\
\hline 664 & 17 & -- & ----- & ----- & 30.28 & no-fit \\
\hline 665 & 31 & 0.50 & $22.07 \pm 0.58$ & $9.37 \pm * * * *$ & 30.75 & hard tail \\
\hline 666 & 31 & 0.71 & $21.78 \pm 0.52$ & $33.31 \pm * * * *$ & 30.56 & hard tail \\
\hline 667 & 46 & 0.51 & $21.76 \pm 0.32$ & $3.94 \pm 3.57$ & 30.72 & fitted \\
\hline 668 & 42 & 0.62 & $21.60 \pm 0.35$ & $1.81 \pm 0.78$ & 30.55 & fitted \\
\hline 669 & 8 & -- & ----- & ----- & 29.94 & no-fit \\
\hline 670 & 82 & 1.21 & $21.92 \pm 0.29$ & $1.19 \pm 0.44$ & 31.02 & fitted \\
\hline 671 & 9 & -- & ----- & ----- & 30.01 & no-fit \\
\hline 672 & 11 & -- & ----- & ----- & 30.09 & no-fit \\
\hline 673 & 98 & 0.74 & $21.84 \pm 0.68$ & $5.73 \pm 4.48$ & 30.83 & fitted \\
\hline 674 & 78 & 0.45 & $21.66 \pm 0.24$ & $2.41 \pm 1.06$ & 30.89 & fitted \\
\hline 675 & 29 & 0.69 & $22.25 \pm 0.32$ & $2.81 \pm 2.75$ & 30.67 & fitted \\
\hline 676 & 12 & -- & ----- & ----- & 30.13 & no-fit \\
\hline 677 & 49 & 0.66 & $21.65 \pm 0.39$ & $4.97 \pm 4.75$ & 30.70 & fitted \\
\hline 678 & 8 & -- & ----- & ----- & 29.95 & no-fit \\
\hline 679 & 11 & -- & ----- & ----- & 32.47 & fitted \\
\hline 680 & 52 & 0.65 & $21.76 \pm 0.34$ & $1.42 \pm 0.49$ & 30.69 & fitted \\
\hline 681 & 49 & 0.66 & $21.08 \pm 0.68$ & $3.52 \pm 3.00$ & 30.53 & fitted \\
\hline 682 & 117 & 1.04 & $21.14 \pm 0.40$ & $3.86 \pm 2.30$ & 31.10 & no-fit \\
\hline 683 & 23 & 0.54 & $22.01 \pm 0.43$ & $1.82 \pm 1.31$ & 32.15 & fitted \\
\hline 684 & 11 & -- & ----- & ----- & 30.08 & no-fit \\
\hline 685 & 15 & -- & ----- & ----- & 30.21 & no-fit \\
\hline 686 & 16 & -- & ----- & ----- & 32.55 & fitted \\
\hline 687 & 217 & 0.72 & $21.66 \pm 0.95$ & $0.62 \pm 0.65$ & 31.54 & $\operatorname{Tr} 16-23$ \\
\hline 688 & 82 & 0.81 & $21.58 \pm 0.19$ & $0.64 \pm 0.11$ & 31.38 & Tr16-9 \\
\hline 689 & 1718 & 1.95 & $21.45 \pm 0.53$ & $0.56 \pm 0.17$ & 32.25 & HDE303308 \\
\hline 690 & 9 & -- & ----- & ----- & 30.01 & no-fit \\
\hline 691 & 75 & 1.13 & $21.11 \pm 0.54$ & $2.39 \pm 0.88$ & 30.67 & fitted \\
\hline 692 & 29 & 0.62 & $20.83 \pm 1.50$ & $7.87 \pm * * * *$ & 30.29 & fitted \\
\hline 693 & 60 & 0.74 & $21.25 \pm 0.58$ & $8.93 \pm * * * *$ & 30.82 & hard tail \\
\hline 694 & 23 & 0.35 & $21.73 \pm 0.64$ & $1.37 \pm 0.66$ & 30.34 & fitted \\
\hline 695 & 44 & 0.96 & $21.78 \pm 0.10$ & $1.42 \pm 0.41$ & 30.28 & fitted \\
\hline 696 & 434 & 1.27 & $21.48 \pm 1.19$ & $7.47 \pm 2.96$ & 31.80 & fitted \\
\hline 697 & 8 & -- & ----- & ----- & 29.93 & no-fit \\
\hline 698 & 45 & 0.94 & $21.06 \pm 0.87$ & $2.59 \pm 1.44$ & 30.55 & fitted \\
\hline 699 & 28 & 0.75 & $22.43 \pm 0.57$ & $64.00 \pm * * * *$ & 30.90 & hard tail \\
\hline 700 & 29 & -- & ----- & ----- & 30.50 & no-fit \\
\hline 701 & 7 & -- & ----- & ----- & 29.92 & no-fit \\
\hline 702 & 5 & -- & ----- & ----- & 29.74 & no-fit \\
\hline 703 & 20 & 0.59 & $22.53 \pm 0.59$ & $1.62 \pm 2.12$ & 30.83 & fitted \\
\hline 704 & 10 & -- & ----- & ----- & 30.06 & no-fit \\
\hline 705 & 14 & -- & ----- & ----- & 30.19 & no-fit \\
\hline 706 & 9 & -- & ----- & ----- & 30.01 & no-fit \\
\hline 707 & 91 & 0.85 & $21.34 \pm 0.55$ & $0.36 \pm 0.14$ & 30.97 & $\operatorname{Tr} 16-3$ \\
\hline 708 & 11 & -- & ----- & ----- & 30.10 & no-fit \\
\hline 709 & 71 & 0.61 & $21.59 \pm 0.28$ & $2.98 \pm 1.66$ & 30.82 & fitted \\
\hline 710 & 8 & -- & ----- & ----- & 29.98 & no-fit \\
\hline 711 & 5 & -- & ----- & ----- & 29.76 & no-fit \\
\hline 712 & 12 & -- & ----- & ----- & 30.31 & no-fit \\
\hline 713 & 14 & -- & ----- & ----- & 30.18 & no-fit \\
\hline 714 & 201 & 1.87 & $21.11 \pm 2.94$ & $5.35 \pm 2.49$ & 31.16 & fitted \\
\hline 715 & 30 & 0.45 & $21.48 \pm 0.55$ & $1.29 \pm 0.35$ & 30.64 & fitted \\
\hline
\end{tabular}

Table 3. continued.

\begin{tabular}{|c|c|c|c|c|c|c|}
\hline $\begin{array}{l}N_{x} \\
\#\end{array}$ & $\begin{array}{l}\text { Cnts. } \\
\text { (ph) }\end{array}$ & $\begin{array}{l}\text { Stat. } \\
\left(\chi_{v}^{2}\right)\end{array}$ & $\begin{array}{l}\log \left(N_{\mathrm{H}}\right) \\
\left(\mathrm{cm}^{-2}\right)\end{array}$ & $\begin{array}{l}k T \\
(\mathrm{keV})\end{array}$ & $\begin{array}{l}\log \left(L_{\mathrm{x}}\right) \\
(\mathrm{erg} / \mathrm{s})\end{array}$ & flag \\
\hline 716 & 24 & 0.77 & $21.38 \pm 0.69$ & $1.72 \pm 1.08$ & 30.21 & fitted \\
\hline 717 & 23 & 0.93 & $21.77 \pm 1.17$ & $1.64 \pm 2.26$ & 30.29 & fitted \\
\hline 718 & 34 & 0.56 & $21.69 \pm 0.47$ & $1.17 \pm 0.41$ & 30.45 & fitted \\
\hline 719 & 4 & -- & ----- & ----- & 29.70 & no-fit \\
\hline 720 & 17 & -- & ----- & ----- & 30.27 & no-fit \\
\hline 721 & 20 & 0.62 & $22.29 \pm 0.46$ & $1.09 \pm 1.09$ & 30.90 & fitted \\
\hline 722 & 54 & 1.04 & $22.49 \pm 0.30$ & $12.65 \pm * * * *$ & 31.21 & hard tail \\
\hline 723 & 24 & 0.42 & $21.68 \pm 0.76$ & $1.64 \pm 1.14$ & 30.33 & fitted \\
\hline 724 & 59 & 1.00 & $22.21 \pm 0.24$ & $1.46 \pm 0.58$ & 31.15 & fitted \\
\hline 725 & 187 & 2.04 & $21.38 \pm 1.98$ & $3.00 \pm 0.96$ & 31.14 & fitted \\
\hline 726 & 26 & 1.08 & $22.13 \pm 0.66$ & $1.29 \pm 1.24$ & 30.66 & fitted \\
\hline 727 & 59 & 0.63 & $21.58 \pm 0.36$ & $2.16 \pm 0.85$ & 30.71 & fitted \\
\hline 728 & 7 & -- & ----- & ----- & 29.91 & no-fit \\
\hline 729 & 56 & 0.90 & $21.62 \pm 0.36$ & $1.92 \pm 0.74$ & 30.40 & CPD-592628B \\
\hline 730 & 2029 & 1.57 & $21.68 \pm 0.43$ & $1.86 \pm 0.98$ & 32.31 & $\operatorname{Tr} 16-22$ \\
\hline 731 & 109 & 0.45 & $21.23 \pm 0.38$ & $3.26 \pm 1.21$ & 30.99 & fitted \\
\hline 732 & 15 & -- & ----- & ----- & 30.23 & no-fit \\
\hline 733 & 21 & 0.65 & $21.95 \pm 0.69$ & $1.22 \pm 1.03$ & 30.46 & fitted \\
\hline 734 & 177 & 1.26 & $21.73 \pm 0.57$ & $2.11 \pm 0.31$ & 31.02 & fitted \\
\hline 735 & 48 & 0.45 & $21.54 \pm 0.38$ & $2.91 \pm 1.91$ & 30.62 & fitted \\
\hline 736 & 34 & 0.56 & $21.62 \pm 0.50$ & $1.44 \pm 0.62$ & 30.43 & fitted \\
\hline 737 & 6 & -- & ----- & ----- & 29.83 & no-fit \\
\hline 738 & 33 & 0.82 & $21.63 \pm 0.72$ & $1.37 \pm 0.79$ & 30.33 & fitted \\
\hline 739 & 3 & -- & ----- & ----- & 28.92 & no-fit \\
\hline 740 & 7 & -- & ----- & ----- & 29.93 & no-fit \\
\hline 741 & 33 & 0.49 & $22.24 \pm 0.22$ & $1.44 \pm 0.66$ & 30.89 & fitted \\
\hline 742 & 3 & -- & ---- & ----- & 28.92 & no-fit \\
\hline 743 & 22 & 0.26 & $21.99 \pm 0.22$ & $0.80 \pm 0.41$ & 30.77 & fitted \\
\hline 744 & 3 & -- & ----- & ----- & 28.92 & no-fit \\
\hline 745 & 5 & -- & ----- & ----- & 29.75 & no-fit \\
\hline 746 & 61 & 1.86 & $21.36 \pm 0.60$ & $64.00 \pm * * * *$ & 30.91 & hard tail \\
\hline 747 & 9 & -- & ----- & ----- & 30.02 & no-fit \\
\hline 748 & 44 & 0.79 & $21.46 \pm 0.61$ & $1.44 \pm 0.55$ & 30.50 & fitted \\
\hline 749 & 45 & 1.19 & $22.22 \pm 0.29$ & $2.33 \pm 1.63$ & 30.92 & fitted \\
\hline 750 & 12 & -- & ----- & ----- & 30.12 & no-fit \\
\hline 751 & 13 & -- & ----- & ----- & 30.16 & no-fit \\
\hline 752 & 17 & -- & ----- & ----- & 30.28 & no-fit \\
\hline 753 & 68 & 1.07 & $21.13 \pm 0.54$ & $1.53 \pm 0.34$ & 30.56 & fitted \\
\hline 754 & 10 & -- & ----- & ----- & 30.04 & no-fit \\
\hline 755 & 68 & 0.88 & $21.93 \pm 0.18$ & $0.91 \pm 0.24$ & 31.07 & fitted \\
\hline 756 & 76 & 0.87 & $21.32 \pm 0.35$ & $1.93 \pm 0.61$ & 30.70 & fitted \\
\hline 757 & 5 & -- & ----- & ----- & 29.79 & no-fit \\
\hline 758 & 9 & -- & ----- & ----- & 30.21 & no-fit \\
\hline 759 & 7 & -- & ----- & ----- & 29.90 & $\operatorname{Tr} 16-74$ \\
\hline 760 & 13 & -- & ----- & ----- & 30.17 & no-fit \\
\hline 761 & 121 & 0.78 & $21.59 \pm 0.25$ & $8.99 \pm 9.26$ & 31.16 & hard tail \\
\hline 762 & 67 & 1.51 & $21.77 \pm 0.35$ & $3.20 \pm 2.47$ & 30.82 & fitted \\
\hline 763 & 43 & 1.30 & $21.87 \pm 0.25$ & $0.76 \pm 0.33$ & 30.75 & fitted \\
\hline 764 & 31 & 0.53 & $22.09 \pm 0.41$ & $1.67 \pm 1.15$ & 30.91 & fitted \\
\hline 765 & 10 & -- & ----- & ----- & 30.06 & no-fit \\
\hline 766 & 35 & 0.88 & $22.56 \pm 0.27$ & $1.64 \pm 1.03$ & 31.29 & fitted \\
\hline 767 & 24 & 0.72 & $21.87 \pm 0.24$ & $3.19 \pm 4.64$ & 30.19 & fitted \\
\hline 768 & 22 & 0.93 & $22.37 \pm 0.50$ & $1.37 \pm 1.30$ & 30.73 & fitted \\
\hline 769 & 60 & 1.12 & $21.40 \pm 0.41$ & $1.45 \pm 0.39$ & 30.68 & fitted \\
\hline 770 & 22 & 0.59 & $21.94 \pm 0.40$ & $1.30 \pm 0.71$ & 30.56 & fitted \\
\hline 771 & 19 & -- & ----- & ----- & 30.32 & no-fit \\
\hline 772 & 5 & -- & ----- & ----- & 29.79 & no-fit \\
\hline 773 & 22 & 0.37 & $21.75 \pm 0.53$ & $1.78 \pm 1.38$ & 30.38 & fitted \\
\hline 774 & 4 & -- & ----- & ----- & 29.69 & no-fit \\
\hline 775 & 34 & 0.75 & $21.81 \pm 0.12$ & $2.65 \pm 1.26$ & 30.34 & fitted \\
\hline 776 & 10 & -- & ----- & ----- & 30.05 & no-fit \\
\hline 777 & 8 & -- & ----- & ----- & 29.95 & no-fit \\
\hline 778 & 25 & 0.65 & $21.53 \pm 1.12$ & $1.55 \pm 1.10$ & 30.25 & fitted \\
\hline 779 & 89 & 0.67 & $21.51 \pm 0.28$ & $4.63 \pm 3.54$ & 30.97 & fitted \\
\hline 780 & 48 & 0.74 & $21.78 \pm 0.27$ & $0.86 \pm 0.29$ & 30.80 & fitted \\
\hline
\end{tabular}


Table 3. continued.

\begin{tabular}{|c|c|c|c|c|c|c|}
\hline $\begin{array}{l}N_{x} \\
\#\end{array}$ & $\begin{array}{l}\text { Cnts. } \\
(\mathrm{ph})\end{array}$ & $\begin{array}{l}\text { Stat. } \\
\left(\chi_{v}^{2}\right)\end{array}$ & $\begin{array}{l}\log \left(N_{\mathrm{H}}\right) \\
\left(\mathrm{cm}^{-2}\right)\end{array}$ & $\begin{array}{l}k T \\
(\mathrm{keV})\end{array}$ & $\begin{array}{l}\log \left(L_{\mathrm{x}}\right) \\
(\mathrm{erg} / \mathrm{s}) \\
\end{array}$ & flag \\
\hline 781 & 55 & 0.85 & $22.54 \pm 0.27$ & $64.00 \pm * * * *$ & 31.13 & hard tail \\
\hline 782 & 9 & -- & ----- & ----- & 30.00 & no-fit \\
\hline 783 & 11 & -- & ----- & ----- & 30.08 & no-fit \\
\hline 784 & 188 & 1.02 & $21.76 \pm 0.14$ & $2.86 \pm 0.73$ & 31.34 & fitted \\
\hline 785 & 12 & -- & ----- & ----- & 30.12 & no-fit \\
\hline 786 & 84 & 0.87 & $21.65 \pm 0.31$ & $1.49 \pm 0.44$ & 30.85 & fitted \\
\hline 787 & 68 & 1.24 & $21.57 \pm 0.35$ & $1.48 \pm 0.45$ & 30.70 & fitted \\
\hline 788 & 98 & 1.10 & $21.42 \pm 0.26$ & $1.98 \pm 0.47$ & 30.89 & fitted \\
\hline 789 & 8 & -- & ----- & ----- & 30.03 & no-fit \\
\hline 790 & 7 & -- & ----- & ----- & 29.92 & no-fit \\
\hline 791 & 29 & 0.60 & $21.40 \pm 0.97$ & $4.18 \pm 4.98$ & 30.38 & fitted \\
\hline 792 & 46 & 0.41 & $21.92 \pm 0.21$ & $1.06 \pm 0.28$ & 30.85 & fitted \\
\hline 793 & 250 & 0.91 & $21.85 \pm 0.11$ & $3.73 \pm 1.13$ & 31.60 & fitted \\
\hline 794 & 40 & 0.33 & $22.06 \pm 0.33$ & $1.55 \pm 0.84$ & 30.87 & fitted \\
\hline 795 & 15 & -- & ----- & ----- & 30.21 & no-fit \\
\hline 796 & 27 & 0.64 & $22.68 \pm 0.35$ & $1.54 \pm 1.27$ & 31.27 & fitted \\
\hline 797 & 8 & -- & ----- & ----- & 29.93 & no-fit \\
\hline 798 & 31 & 0.45 & $22.10 \pm 0.19$ & $0.86 \pm 0.43$ & 31.03 & fitted \\
\hline 799 & 4 & -- & ----- & ----- & 29.69 & no-fit \\
\hline 800 & 8 & -- & ----- & ----- & 30.13 & no-fit \\
\hline 801 & 15 & -- & ----- & ----- & 30.20 & no-fit \\
\hline 802 & 19 & -- & ----- & ----- & 30.31 & no-fit \\
\hline 803 & 504 & 2.88 & $21.55 \pm 0.51$ & $1.38 \pm 0.11$ & 31.38 & HD93343 \\
\hline 804 & 11 & -- & ----- & ----- & 30.26 & no-fit \\
\hline 805 & 12 & -- & ----- & ----- & 30.12 & no-fit \\
\hline 806 & 60 & 1.02 & $21.26 \pm 0.46$ & $2.92 \pm 2.75$ & 30.82 & fitted \\
\hline 807 & 14 & -- & ----- & ----- & 30.20 & Tr16-76 \\
\hline 808 & 393 & 0.67 & $21.40 \pm 1.41$ & $0.69 \pm 0.52$ & 31.53 & CPD-592635B \\
\hline 809 & 20 & 1.01 & $21.73 \pm 0.43$ & $1.87 \pm 1.22$ & 29.87 & fitted \\
\hline 810 & 16 & -- & ----- & ----- & 30.24 & no-fit \\
\hline 811 & 631 & 1.43 & $22.07 \pm 0.07$ & $10.85 \pm 6.83$ & 32.07 & hard tail \\
\hline 812 & 606 & 0.37 & $21.65 \pm 0.57$ & $0.62 \pm 0.37$ & 31.93 & CPD-592636C \\
\hline 813 & 13 & -- & ----- & ----- & 30.16 & no-fit \\
\hline 814 & 11 & -- & ----- & ----- & 30.10 & no-fit \\
\hline 815 & 8 & -- & ----- & ----- & 29.94 & no-fit \\
\hline 816 & 90 & 0.73 & $21.59 \pm 0.43$ & $2.28 \pm 1.13$ & 30.94 & fitted \\
\hline 817 & 11 & -- & ----- & ----- & 30.07 & no-fit \\
\hline 818 & 13 & -- & ----- & ----- & 30.15 & no-fit \\
\hline 819 & 42 & 1.14 & $21.17 \pm 0.90$ & $5.85 \pm * * * *$ & 30.46 & fitted \\
\hline 820 & 10 & -- & ----- & ----- & 30.03 & no-fit \\
\hline 821 & 37 & 1.07 & $21.79 \pm 0.62$ & $1.18 \pm 0.75$ & 30.54 & fitted \\
\hline 822 & 80 & 0.82 & $21.23 \pm 0.43$ & $3.94 \pm 2.52$ & 30.79 & fitted \\
\hline 823 & 51 & 0.94 & $21.85 \pm 0.31$ & $1.53 \pm 0.48$ & 30.41 & fitted \\
\hline 824 & 12 & -- & ----- & ----- & 30.13 & no-fit \\
\hline 825 & 10 & -- & ----- & ----- & 30.04 & no-fit \\
\hline 826 & 30 & 0.86 & $22.07 \pm 0.62$ & $2.58 \pm 3.56$ & 30.59 & fitted \\
\hline 827 & 9 & -- & ----- & ----- & 30.01 & no-fit \\
\hline 828 & 14 & -- & ----- & ----- & 30.20 & no-fit \\
\hline 829 & 5 & -- & ----- & ----- & 29.80 & no-fit \\
\hline 830 & 38 & 0.72 & $21.04 \pm 0.77$ & $3.26 \pm 2.98$ & 30.61 & fitted \\
\hline 831 & 39 & 0.80 & $21.73 \pm 0.14$ & $2.68 \pm 1.42$ & 30.36 & fitted \\
\hline 832 & 152 & 1.14 & $21.10 \pm 0.41$ & $10.03 \pm 8.70$ & 31.13 & hard tail \\
\hline 833 & 29 & 1.58 & $22.03 \pm 0.51$ & $1.09 \pm 0.91$ & 30.64 & fitted \\
\hline 834 & 67 & 0.72 & $21.50 \pm 0.32$ & $3.03 \pm 1.50$ & 30.75 & fitted \\
\hline 835 & 22 & 0.68 & $21.41 \pm 1.04$ & $3.84 \pm 6.22$ & 30.14 & fitted \\
\hline 836 & 21 & 0.37 & $21.13 \pm 1.22$ & $43.50 \pm * * * *$ & 30.51 & hard tail \\
\hline 837 & 13 & -- & ----- & ----- & 30.15 & no-fit \\
\hline 838 & 41 & 0.59 & $22.10 \pm 0.33$ & $1.23 \pm 0.65$ & 30.89 & fitted \\
\hline 839 & 90 & 0.94 & $21.97 \pm 0.23$ & $1.91 \pm 0.90$ & 31.08 & fitted \\
\hline 840 & 11 & -- & ----- & ----- & 30.29 & no-fit \\
\hline 841 & 28 & 1.03 & $21.53 \pm 1.20$ & $1.37 \pm 0.96$ & 30.18 & fitted \\
\hline 842 & 12 & -- & ----- & ----- & 30.14 & no-fit \\
\hline 843 & 19 & -- & ----- & ----- & 30.31 & no-fit \\
\hline 844 & 15 & -- & ----- & ----- & 30.21 & no-fit \\
\hline 845 & 44 & 0.46 & $21.52 \pm 0.19$ & $1.72 \pm 0.45$ & 30.38 & fitted \\
\hline 846 & 15 & -- & ----- & ----- & 30.21 & no-fit \\
\hline
\end{tabular}

Table 3. continued.

\begin{tabular}{|c|c|c|c|c|c|c|}
\hline $\begin{array}{l}N_{x} \\
\#\end{array}$ & $\begin{array}{l}\text { Cnts. } \\
\text { (ph) }\end{array}$ & $\begin{array}{l}\text { Stat. } \\
\left(\chi_{v}^{2}\right)\end{array}$ & $\begin{array}{l}\log \left(N_{\mathrm{H}}\right) \\
\left(\mathrm{cm}^{-2}\right)\end{array}$ & $\begin{array}{l}k T \\
(\mathrm{keV})\end{array}$ & $\begin{array}{l}\log \left(L_{\mathrm{x}}\right) \\
(\mathrm{erg} / \mathrm{s})\end{array}$ & flag \\
\hline 847 & 90 & 0.72 & $21.73 \pm 0.27$ & $1.55 \pm 0.42$ & 32.02 & fitted \\
\hline 848 & 151 & 1.62 & $21.41 \pm 0.22$ & $2.01 \pm 0.42$ & 31.00 & fitted \\
\hline 849 & 47 & 0.66 & $21.66 \pm 0.18$ & $3.77 \pm 3.55$ & 30.61 & fitted \\
\hline 850 & 3 & -- & ----- & ----- & 29.61 & no-fit \\
\hline 851 & 7 & -- & ----- & ----- & 29.87 & no-fit \\
\hline 852 & 22 & 0.58 & $21.87 \pm 0.59$ & $7.76 \pm * * * *$ & 30.51 & fitted \\
\hline 853 & 34 & 0.75 & $21.18 \pm 0.59$ & $1.84 \pm 0.87$ & 30.25 & fitted \\
\hline 854 & 645 & 0.90 & $21.88 \pm 0.47$ & $0.37 \pm 0.36$ & 32.44 & CPD-592641 \\
\hline 855 & 29 & -- & ----- & ----- & 30.49 & no-fit \\
\hline 856 & 14 & -- & ----- & ----- & 30.19 & no-fit \\
\hline 857 & 45 & 0.95 & $21.88 \pm 0.48$ & $1.56 \pm 0.89$ & 30.63 & fitted \\
\hline 858 & 26 & 0.65 & $21.20 \pm 1.02$ & $2.16 \pm 1.63$ & 30.20 & fitted \\
\hline 859 & 82 & 0.88 & $21.20 \pm 0.61$ & $0.49 \pm 0.19$ & 30.80 & fitted \\
\hline 860 & 11 & -- & ----- & ----- & 30.07 & no-fit \\
\hline 861 & 44 & 0.99 & $22.10 \pm 0.34$ & $1.43 \pm 1.01$ & 30.84 & fitted \\
\hline 862 & 50 & 0.93 & $21.86 \pm 0.40$ & $8.28 \pm * * * *$ & 30.79 & hard tail \\
\hline 863 & 31 & 1.05 & $21.98 \pm 0.59$ & $6.28 \pm * * * *$ & 30.67 & fitted \\
\hline 864 & 276 & 0.55 & $21.73 \pm 0.12$ & $3.78 \pm 1.01$ & 31.52 & fitted \\
\hline 865 & 22 & 0.69 & $22.80 \pm 0.40$ & $1.23 \pm 1.23$ & 31.43 & fitted \\
\hline 866 & 43 & 1.04 & $21.64 \pm 0.47$ & $63.24 \pm * * * *$ & 30.55 & hard tail \\
\hline 867 & 34 & 0.75 & $21.86 \pm 0.12$ & $2.97 \pm 2.39$ & 30.30 & fitted \\
\hline 868 & 39 & 0.77 & $20.98 \pm 2.00$ & $1.78 \pm 0.90$ & 30.25 & fitted \\
\hline 869 & 12 & -- & ----- & ----- & 30.11 & no-fit \\
\hline 870 & 17 & -- & ----- & ----- & 30.28 & no-fit \\
\hline 871 & 62 & 0.58 & $21.59 \pm 0.36$ & $1.03 \pm 0.20$ & 30.91 & fitted \\
\hline 872 & 57 & 0.98 & $21.99 \pm 0.30$ & $1.20 \pm 0.45$ & 31.02 & fitted \\
\hline 873 & 10 & -- & ----- & ----- & 30.03 & no-fit \\
\hline 874 & 41 & 0.95 & $21.24 \pm 0.58$ & $1.68 \pm 0.41$ & 30.35 & fitted \\
\hline 875 & 3 & -- & ----- & ----- & 29.52 & no-fit \\
\hline 876 & 24 & 1.13 & $21.62 \pm 1.69$ & $3.75 \pm 9.68$ & 29.79 & fitted \\
\hline 877 & 57 & 1.08 & $21.91 \pm 0.28$ & $1.17 \pm 0.39$ & 30.92 & fitted \\
\hline 878 & 3 & -- & ----- & ----- & 28.92 & no-fit \\
\hline 879 & 40 & 0.74 & $21.59 \pm 0.43$ & $1.98 \pm 0.85$ & 30.61 & fitted \\
\hline 880 & 21 & 0.40 & $21.76 \pm 0.69$ & $1.72 \pm 1.29$ & 30.33 & fitted \\
\hline 881 & 15 & -- & ----- & ----- & 30.22 & no-fit \\
\hline 882 & 21 & 0.82 & $23.02 \pm 0.49$ & $0.66 \pm 1.18$ & 32.43 & fitted \\
\hline 883 & 31 & 0.24 & $21.91 \pm 0.68$ & $2.12 \pm 2.10$ & 30.61 & fitted \\
\hline 884 & 63 & 0.91 & $21.81 \pm 0.26$ & $0.97 \pm 0.27$ & 30.89 & fitted \\
\hline 885 & 18 & -- & ----- & ----- & 30.30 & no-fit \\
\hline 886 & 60 & 0.34 & $21.09 \pm 0.66$ & $2.41 \pm 0.98$ & 30.67 & fitted \\
\hline 887 & 4 & -- & ----- & ----- & 29.63 & no-fit \\
\hline 888 & 71 & 0.74 & $21.48 \pm 0.26$ & $0.57 \pm 0.10$ & 31.05 & $\operatorname{Tr} 16-115$ \\
\hline 889 & 17 & -- & ----- & ----- & 30.27 & no-fit \\
\hline 890 & 50 & 0.76 & $21.57 \pm 0.47$ & $12.50 \pm * * * *$ & 30.69 & hard tail \\
\hline 891 & 14 & -- & ----- & ----- & 30.19 & no-fit \\
\hline 892 & 28 & 0.68 & $21.48 \pm 0.56$ & $0.79 \pm 0.25$ & 30.35 & fitted \\
\hline 893 & 15 & -- & ----- & ----- & 30.20 & no-fit \\
\hline 894 & 56 & 1.02 & $21.67 \pm 0.56$ & $63.99 \pm * * * *$ & 30.91 & hard tail \\
\hline 895 & 10 & -- & ----- & ----- & 30.04 & no-fit \\
\hline 896 & 40 & 0.38 & $21.56 \pm 0.46$ & $1.72 \pm 0.62$ & 30.52 & fitted \\
\hline 897 & 12 & -- & ----- & ----- & 30.12 & no-fit \\
\hline 898 & 24 & 0.59 & $21.74 \pm 0.68$ & $31.71 \pm * * * *$ & 30.48 & hard tail \\
\hline 899 & 3 & -- & ----- & ----- & 29.63 & no-fit \\
\hline 900 & 8 & -- & ----- & ----- & 29.94 & no-fit \\
\hline 901 & 10 & -- & ----- & ----- & 30.04 & no-fit \\
\hline 902 & 119 & 1.52 & $21.79 \pm 0.21$ & $1.90 \pm 0.52$ & 31.19 & fitted \\
\hline 903 & 147 & 0.77 & $21.64 \pm 0.21$ & $1.70 \pm 0.26$ & 31.16 & fitted \\
\hline 904 & 75 & 0.70 & $21.46 \pm 0.39$ & $5.18 \pm 3.37$ & 30.84 & fitted \\
\hline 905 & 75 & 0.80 & $21.14 \pm 0.68$ & $42.89 \pm * * * *$ & 30.90 & hard tail \\
\hline 906 & 52 & 1.29 & $21.94 \pm 0.28$ & $1.09 \pm 0.46$ & 30.91 & fitted \\
\hline 907 & 60 & 0.84 & $22.14 \pm 0.17$ & $0.90 \pm 0.30$ & 31.26 & fitted \\
\hline 908 & 66 & 1.68 & $22.14 \pm 0.24$ & $1.15 \pm 0.44$ & 31.26 & fitted \\
\hline 909 & 194 & 1.27 & $21.90 \pm 0.11$ & $0.87 \pm 0.13$ & 31.63 & fitted \\
\hline 910 & 3 & -- & ----- & ----- & 28.92 & no-fit \\
\hline 911 & 8 & -- & ----- & ----- & 29.95 & no-fit \\
\hline 912 & 28 & 0.50 & $21.53 \pm 0.66$ & $2.49 \pm 1.92$ & 30.35 & fitted \\
\hline
\end{tabular}


Table 3. continued.

\begin{tabular}{|c|c|c|c|c|c|c|}
\hline $\begin{array}{l}N_{x} \\
\#\end{array}$ & $\begin{array}{l}\text { Cnts. } \\
\text { (ph) }\end{array}$ & $\begin{array}{l}\text { Stat. } \\
\left(\chi_{v}^{2}\right)\end{array}$ & $\begin{array}{l}\log \left(N_{\mathrm{H}}\right) \\
\left(\mathrm{cm}^{-2}\right)\end{array}$ & $\begin{array}{l}k T \\
(\mathrm{keV})\end{array}$ & $\begin{array}{l}\log \left(L_{\mathrm{x}}\right) \\
(\mathrm{erg} / \mathrm{s})\end{array}$ & flag \\
\hline 913 & 27 & 0.70 & $21.35 \pm 0.83$ & $0.54 \pm 0.37$ & 30.41 & fitted \\
\hline 914 & 14 & -- & ----- & ----- & 30.20 & no-fit \\
\hline 915 & 7 & -- & ----- & ----- & 29.90 & no-fit \\
\hline 916 & 62 & 0.93 & $21.28 \pm 0.44$ & $1.80 \pm 0.54$ & 30.60 & fitted \\
\hline 917 & 23 & 1.20 & $21.99 \pm 0.31$ & $2.25 \pm 4.25$ & 29.86 & fitted \\
\hline 918 & 7 & -- & ----- & ----- & 29.88 & no-fit \\
\hline 919 & 11 & -- & ----- & ----- & 30.08 & no-fit \\
\hline 920 & 46 & 0.59 & $21.37 \pm 0.56$ & $4.59 \pm 4.13$ & 30.58 & fitted \\
\hline 921 & 8 & -- & ----- & ----- & 29.97 & no-fit \\
\hline 922 & 40 & 0.65 & $22.03 \pm 0.34$ & $1.52 \pm 0.81$ & 30.80 & fitted \\
\hline 923 & 28 & 1.20 & $21.97 \pm 0.55$ & $6.02 \pm * * * *$ & 30.54 & fitted \\
\hline 924 & 89 & 1.00 & $21.91 \pm 0.26$ & $1.32 \pm 0.34$ & 31.10 & fitted \\
\hline 925 & 13 & -- & ----- & ----- & 30.15 & no-fit \\
\hline 926 & 27 & 0.47 & $21.48 \pm 0.61$ & $3.15 \pm 2.86$ & 30.31 & fitted \\
\hline 927 & 8 & -- & ----- & ----- & 29.94 & no-fit \\
\hline 928 & 179 & 1.11 & $21.72 \pm 0.15$ & $2.58 \pm 0.70$ & 31.27 & fitted \\
\hline 929 & 48 & 1.49 & $22.78 \pm 0.44$ & $1.63 \pm 1.62$ & 31.48 & fitted \\
\hline 930 & 9 & -- & ----- & ----- & 30.00 & no-fit \\
\hline 931 & 13 & -- & ----- & ----- & 30.17 & no-fit \\
\hline 932 & 46 & 9.75 & $22.58 \pm 0.23$ & $4.00 \pm 3.90$ & 31.28 & fitted \\
\hline 933 & 118 & 0.52 & $22.15 \pm 0.18$ & $13.70 \pm * * * *$ & 31.37 & hard tail \\
\hline 934 & 71 & 0.49 & $21.78 \pm 0.28$ & $1.82 \pm 0.65$ & 30.91 & fitted \\
\hline 935 & 44 & 1.14 & $21.49 \pm 0.46$ & $1.81 \pm 0.85$ & 30.27 & fitted \\
\hline 936 & 5 & -- & ----- & ----- & 29.93 & no-fit \\
\hline 937 & 30 & -- & ----- & ----- & 30.51 & no-fit \\
\hline 938 & 59 & 0.69 & $22.68 \pm 0.17$ & $1.48 \pm 0.56$ & 31.67 & fitted \\
\hline 939 & 17 & -- & ----- & ----- & 30.26 & no-fit \\
\hline 940 & 16 & -- & ----- & ----- & 30.24 & no-fit \\
\hline 941 & 26 & 0.87 & $21.72 \pm 0.97$ & $63.91 \pm * * * *$ & 30.22 & hard tail \\
\hline 942 & 40 & 0.98 & $22.61 \pm 0.32$ & $5.44 \pm 9.70$ & 31.17 & fitted \\
\hline 943 & 23 & 0.55 & $22.21 \pm 0.33$ & $0.98 \pm 0.68$ & 30.87 & fitted \\
\hline 944 & 39 & 0.98 & $20.43 \pm 5.49$ & $64.00 \pm * * * *$ & 30.62 & hard tail \\
\hline 945 & 6 & -- & ----- & ----- & 29.91 & no-fit \\
\hline 946 & 112 & 0.61 & $21.37 \pm 0.26$ & $2.04 \pm 0.40$ & 31.13 & fitted \\
\hline 947 & 7 & -- & ----- & ----- & 29.92 & no-fit \\
\hline 948 & 27 & 0.40 & $21.95 \pm 0.40$ & $2.19 \pm 1.97$ & 30.61 & fitted \\
\hline 949 & 20 & 0.42 & $21.99 \pm 0.56$ & $1.37 \pm 1.02$ & 30.53 & fitted \\
\hline 950 & 12 & -- & ----- & ----- & 30.11 & no-fit \\
\hline 951 & 15 & -- & ----- & ----- & 30.22 & no-fit \\
\hline 952 & 18 & -- & ----- & ----- & 30.29 & no-fit \\
\hline 953 & 20 & 0.68 & $22.23 \pm 0.34$ & $0.93 \pm 0.62$ & 30.79 & fitted \\
\hline 954 & 55 & 0.65 & $21.63 \pm 0.34$ & $1.79 \pm 0.74$ & 30.69 & fitted \\
\hline 955 & 69 & 1.02 & $21.17 \pm 0.52$ & $2.34 \pm 1.25$ & 30.75 & fitted \\
\hline 956 & 7 & -- & ----- & ----- & 29.99 & no-fit \\
\hline 957 & 47 & 0.61 & $22.32 \pm 0.16$ & $0.74 \pm 0.28$ & 31.58 & fitted \\
\hline 958 & 180 & 1.23 & $21.65 \pm 0.18$ & $2.34 \pm 0.64$ & 31.21 & fitted \\
\hline 959 & 27 & 0.61 & $22.69 \pm 0.39$ & $1.92 \pm 2.14$ & 31.15 & fitted \\
\hline 960 & 12 & -- & ----- & ----- & 30.12 & no-fit \\
\hline 961 & 176 & 0.46 & $21.94 \pm 0.20$ & $2.61 \pm 1.12$ & 31.47 & fitted \\
\hline 962 & 28 & 0.70 & $22.03 \pm 0.26$ & $0.69 \pm 0.45$ & 30.90 & fitted \\
\hline 963 & 26 & 0.78 & $22.35 \pm 0.69$ & $62.47 \pm * * * *$ & 30.65 & hard tail \\
\hline 964 & 12 & -- & ----- & ----- & 30.12 & no-fit \\
\hline 965 & 12 & -- & ----- & ----- & 30.10 & no-fit \\
\hline 966 & 78 & 1.19 & $21.40 \pm 0.92$ & $63.95 \pm * * * *$ & 30.99 & hard tail \\
\hline 967 & 37 & 0.51 & $21.54 \pm 0.59$ & $3.04 \pm 2.67$ & 30.55 & fitted \\
\hline 968 & 52 & 0.63 & $22.10 \pm 0.26$ & $1.91 \pm 0.95$ & 30.95 & fitted \\
\hline 969 & 35 & 0.81 & $21.74 \pm 0.77$ & $7.94 \pm * * * *$ & 30.63 & fitted \\
\hline 970 & 29 & -- & ----- & ----- & 30.49 & no-fit \\
\hline 971 & 215 & 1.75 & $21.91 \pm 0.16$ & $5.31 \pm 2.41$ & 31.48 & fitted \\
\hline 972 & 15 & -- & ----- & ----- & 30.22 & no-fit \\
\hline 973 & 53 & 0.38 & $22.16 \pm 0.37$ & $2.56 \pm 2.73$ & 31.05 & fitted \\
\hline 974 & 30 & 0.81 & $22.40 \pm 0.27$ & $0.90 \pm 0.44$ & 31.42 & fitted \\
\hline 975 & 133 & 0.80 & $22.28 \pm 0.15$ & $4.03 \pm 2.20$ & 31.49 & fitted \\
\hline 976 & 20 & 0.67 & $21.99 \pm 0.94$ & $6.76 \pm * * * *$ & 30.29 & fitted \\
\hline
\end{tabular}

Table 3. continued.

\begin{tabular}{|c|c|c|c|c|c|c|}
\hline $\begin{array}{l}N_{x} \\
\#\end{array}$ & $\begin{array}{l}\text { Cnts. } \\
(\mathrm{ph})\end{array}$ & $\begin{array}{l}\text { Stat. } \\
\left(\chi_{v}^{2}\right)\end{array}$ & $\begin{array}{l}\log \left(N_{\mathrm{H}}\right) \\
\left(\mathrm{cm}^{-2}\right)\end{array}$ & $\begin{array}{l}k T \\
(\mathrm{keV})\end{array}$ & $\begin{array}{l}\log \left(L_{\mathrm{x}}\right) \\
(\mathrm{erg} / \mathrm{s})\end{array}$ & flag \\
\hline 977 & 23 & -- & ----- & ----- & 30.39 & no-fit \\
\hline 978 & 58 & 0.85 & $21.33 \pm 1.21$ & $63.71 \pm * * * *$ & 30.81 & ard tail \\
\hline 979 & 36 & 0.87 & $21.72 \pm 0$ & 21.6 & 30.61 & $\mathrm{~h}$ \\
\hline 980 & 102 & 0.82 & $22.16 \pm 0.21$ & $3.24 \pm 1.76$ & 31.30 & fitted \\
\hline 981 & 29 & -- & ----- & ----- & 30.49 & o-fit \\
\hline 982 & 70 & 0.63 & $22.09 \pm 0.16$ & $0.88 \pm 0.22$ & 31.40 & fitted \\
\hline 983 & 18 & -- & ----- & ----- & 30.29 & no-fit \\
\hline 984 & 17 & -- & ----- & ----- & 30.35 & no-fit \\
\hline 985 & 23 & 0.52 & $22.41 \pm 0.28$ & $1.03 \pm 0.72$ & 31.22 & fitted \\
\hline 986 & 34 & 1.07 & $22.20 \pm 0.33$ & $1.82 \pm 1.32$ & 31.07 & fitted \\
\hline 987 & 58 & 0.65 & $22.11 \pm 0.26$ & $2.78 \pm 2.01$ & 30.98 & fitted \\
\hline 988 & 13 & -- & ----- & ----- & 30.16 & no-fit \\
\hline 989 & 23 & 0.63 & $22.27 \pm 0.53$ & $2.08 \pm 2.59$ & 30.66 & fitted \\
\hline 990 & 36 & 0.99 & $21.75 \pm 0.42$ & $2.57 \pm 1.72$ & 30.24 & fitted \\
\hline 991 & 25 & 0.60 & $22.59 \pm 0.21$ & $0.35 \pm 0.89$ & 32.64 & fitted \\
\hline 992 & 30 & -- & ----- & ----- & 30.51 & no-fit \\
\hline 993 & 234 & 0.57 & $21.74 \pm 0.15$ & $12.89 \pm * * * *$ & 31.52 & hard ta \\
\hline 994 & 28 & -- & ----- & ----- & 30.57 & no-fit \\
\hline 995 & 88 & 1.38 & $22.39 \pm 0.14$ & $1.42 \pm 0.40$ & 31.60 & fitted \\
\hline 996 & 203 & 1.10 & $22.21 \pm 0.06$ & $0.56 \pm 0.71$ & 32.32 & fitted \\
\hline 997 & 273 & 1.01 & $22.03 \pm 0.07$ & $0.94 \pm 0.10$ & 31.87 & fitted \\
\hline 998 & 29 & 0.83 & $22.55 \pm 0.19$ & $0.88 \pm 0.29$ & 31.61 & fitted \\
\hline 999 & 6 & -- & ----- & ----- & 29.92 & no-fit \\
\hline 1000 & 38 & -- & ----- & ----- & 30.61 & no-fit \\
\hline 1001 & 71 & 0.97 & $21.32 \pm 0.72$ & $2.93 \pm 1.87$ & 30.80 & fitted \\
\hline 1002 & 58 & 0.73 & $21.81 \pm 0.36$ & $4.98 \pm 5.27$ & 30.96 & fitted \\
\hline 1003 & 38 & 1.11 & $22.17 \pm 0.41$ & $6.42 \pm * * * *$ & 30.88 & fitted \\
\hline 1004 & 25 & -- & ----- & ----- & 30. & no-fit \\
\hline 1005 & 39 & 1.03 & $3 \pm 0$ & $64.00 \pm * *$ & 30.64 & hard tail \\
\hline 1006 & 193 & 0.72 & $21.48 \pm 0.33$ & $14.00 \pm * * * *$ & 31.42 & hard tail \\
\hline 1007 & 56 & -- & ----- & ----- & 30.77 & no-fit \\
\hline 1008 & 25 & 1.12 & $22.28 \pm 0.50$ & $1.70 \pm 1.69$ & 30.87 & fitted \\
\hline 1009 & 36 & -- & ----- & ----- & 30.58 & no-fit \\
\hline 1010 & 42 & -- & ----- & ----- & 30.65 & no-fit \\
\hline 1011 & 49 & 0.53 & $22.21 \pm 0.29$ & $2.02 \pm 1.18$ & 31.10 & fitted \\
\hline 1012 & 60 & 0.74 & $22.55 \pm 0.19$ & $1.15 \pm 0.44$ & 31.65 & fitted \\
\hline 1013 & 25 & 1.09 & $22.47 \pm 0.33$ & $1.78 \pm 1.39$ & 31.02 & fitted \\
\hline 1014 & 229 & 2.13 & $21.69 \pm 0.16$ & $1.01 \pm 0.69$ & 31.08 & fitted \\
\hline 1015 & 20 & -- & ----- & ----- & 30.43 & no-fit \\
\hline 1016 & 62 & 0.83 & $21.79 \pm 0.60$ & $57.39 \pm * * * *$ & 30.98 & hard ta \\
\hline 1017 & 30 & 0.71 & $21.77 \pm 0.71$ & $5.36 \pm 8.63$ & 30.80 & fitted \\
\hline 1018 & 17 & -- & ----- & ----- & 30.28 & no-fit \\
\hline 1019 & 33 & 0.89 & \pm 0.51 & $1.48 \pm 1.17$ & 30.80 & fitted \\
\hline 1020 & 85 & 1.58 & $22.08 \pm 0.30$ & $1.69 \pm 0.89$ & 31.12 & fitted \\
\hline 1021 & 28 & 0.90 & $22.65 \pm 0.85$ & $64.00 \pm * * * *$ & 30.73 & hard tail \\
\hline 102 & 15 & -- & ----- & ----- & 30.22 & no-fit \\
\hline 102 & 17 & -- & ----- & ----- & 30.28 & no-fit \\
\hline 1024 & 17 & -- & ----- & ----- & 30.27 & no-fit \\
\hline 1025 & 16 & -- & ----- & ----- & 30.25 & no-fit \\
\hline 1026 & 12 & -- & ----- & ----- & 30.11 & no-fit \\
\hline 1027 & 40 & 1.83 & $22.72 \pm 0.30$ & $0.70 \pm 0.33$ & 31.96 & fitted \\
\hline 1028 & 34 & -- & ----- & ----- & 30.56 & no-fit \\
\hline 1029 & 94 & 0.66 & $22.07 \pm 0.21$ & $2.19 \pm 0.96$ & 31.30 & fitted \\
\hline 1030 & 78 & 1.12 & $22.02 \pm 0.36$ & $1.53 \pm 0.76$ & 31.12 & fitted \\
\hline 1031 & 55 & -- & ----- & ----- & 30.77 & no-fit \\
\hline 1032 & 26 & -- & ----- & ----- & 30.44 & no-fit \\
\hline 103 & 34 & -- & ----- & ----- & 30.56 & no-fit \\
\hline 1034 & 29 & -- & ----- & ----- & 30.49 & no-fit \\
\hline 1035 & 89 & 0.62 & $21.56 \pm 0.63$ & $63.96 \pm * * * *$ & 31.12 & hard tail \\
\hline
\end{tabular}

